\title{
Transcriptional Responses to Social Isolation: Characterization of the Neuropeptide Parathyroid Hormone 2
}

\author{
Dissertation \\ zur Erlangung des Doktorgrades \\ der Naturwissenschaften \\ vorgelegt beim Fachbereich Biowissenschaften \\ der Johann Wolfgang Goethe-Universität \\ in Frankfurt am Main \\ von \\ Lukas Anneser \\ aus Bamberg
}

Frankfurt 2020

(D 30) 
vom Fachbereich Biowissenschaften der

Johann Wolfgang Goethe - Universität als Dissertation angenommen.

Dekan: Prof. Dr. Sven Klimpel

Gutachter: Prof. Dr. Erin M. Schuman, Prof. Dr. Virginie Lecaudey

Datum der Disputation: 
Parts of the results presented in this thesis have been published in the following form:

L. Anneser, I. C. Alcantara, A. Gemmer, K. Mirkes, S. Ryu, and E. M. Schuman, The neuropeptide Pth2 dynamically senses others via mechanosensation, Nature, 588 (7839), $653-657$. 


\section{Meinen Eltern}




\section{Acknowledgements}

As the famous saying goes, as scientists we are standing on the shoulders of giants, who enable us to ask meaningful and important questions and make the discoveries hopefully contributing to finding an answer. Additionally, we stand on giant piles of books and papers and are tremendously helped along the way by family, friends, and colleagues. In this section, I want to take the opportunity to highlight the people who helped me to navigate the PhD and make the project a success.

First and foremost, I want to thank my mentor, Erin Schuman, for the guidance along the way. She helped to focus on the important questions and pushed the project to the next level. Of course, many more people deserve to be mentioned and thanked:

My dear colleague and collaborator, Anja Gemmer, who tought me a lot about zebrafish and the techniques necessary to work with this model system. With her drive and passion, she was a great inspiration. Her contributions to the data presented here are hard to overstate.

My previous student, Ivan Alcantara, who shared some of the most exciting times in this project with me. Our discussions, scientific or otherwise, enriched my time in the lab.

Soojin Ryu, our long-term collaborator, who provided knowledge, insights, and materials to the project outlined in this thesis.

The other students I had the pleasure to supervise, first and foremost Kristina Mirkes, who provided essential contributions to this project. Megan Boles, a junior scholar who initially worked with me for only a short period but then returned out of interest for the project. Stephanie Chanda, a student who worked with me during the revision period and always kept up the pace, also under time pressure. They were all marvelous people to work with and I am grateful to have been given this opportunity.

My thesis advisory committee, Virginie Lecaudey, Gilles Laurent, and Kathi Zarnack, 
who gave critical advice and accompanied the project the entire time.

The imaging facility, in particular Florian Vollrath and Dr. Claudio Polisseni, who were invaluably helpful by establishing several of the imaging setups described in this thesis.

The mechanical workshop, in particular Andreas Umminger, Guido Schmalbach, and Fabian Bayer, who printed, glued, and screwed everything together I asked them for.

Dr. Friedrich Kretzschmer and Dr. Georgi Tushev from the scientific computing facility helped me to make sense of analysis pipelines and data structures. They have established an infrastructure at the institute that greatly facilitates data analysis, which is hard to be thankful enough for.

Norman Heller and Erik Papushin built the electric circuitry for the experimental setups described in this thesis. Their professional can-do attitude was enormously helpful.

From the Schuman department, Mantian Wang, Caspar Glock, and Julio Perez offered invaluable insights and taught me necessary technical skills.

I am also grateful to my great colleagues Teresa Spano, Paul Donlin-Asp, Stefano Giandomenico, Mara Müller and Eleanor Vail - they made the institute a better and more cheerful place for me.

Without administrational support, everything would have been way harder. For this, I want to explicitly thank Anita Kula, Nicole Horny, Sara Gil-Mast and Susanne tom Dieck.

Last but not least, I want to thank my friends and family - their support made it all possible. 


\section{Zusammenfassung}

Das Leben in sozialen Gruppen ist charakteristisch für zahlreiche Arten und bestimmt vielfältige Aspekte ihres Verhaltens. Die Einordnung in hierarchische Gefüge, der Versuch, sich fortzupflanzen, das gemeinsame Suchen nach Nahrung und die Abwehr von Raubtieren, um nur wenige Beispiele zu nennen, erfordert vom einzelnen Tier die Anpassung an die jeweilige soziale Situation. Dafür ist es erforderlich, dass der soziale Kontext wahrgenommen und verstanden wird. Eine logische Erweiterung dieses Gedankens ist es, dass auch die Abwesenheit von Artgenossen eine bestimmte soziale Situation darstellt und ein Tier vor spezifische Herausforderungen stellen kann.

Es ist bekannt, dass soziale Isolation zu einer Aktivierung der Stressachse führen kann, ebenso wurde in verschiedenen Vertebraten gezeigt, dass Isolationsbedingungen mit einer verstärkten Aggressionsbereitschaft einhergehen. Bislang ist noch nicht detailliert gezeigt worden, wie genau die Anwesenheit oder Abwesenheit von Artgenossen Tiere auf der physiologischen oder genetischen Ebene beeinflussen kann. In der vorliegenden Arbeit wird der Einfluss, den soziale Isolation auf die Regulation von Genexpression hat, untersucht.

\section{Einfluss sozialer Isolation auf die neuronale Genexpression}

Zu diesem Zweck wurden Zebrafische in verschiedenen Konditionen herangezogen. Zebrafische stammen aus der Familie der Karpfenfische und bilden natürlicherweise Schwärme mit Artgenossen. Sie sind vergleichsweise klein, sodass sie in groer Zahl gehalten werden knnen, was es mglich macht, stark unterschiedliche soziale Konditionen zu untersuchen. Ein weiterer Vorteil dieses Organismus ist es, dass er seit vielen Jahren gut in Laboratorien etabliert ist und sein Genom exakt beschrieben wurde. Dies ermglicht es, Genexpressionsstudien durchzufhren und mit hoher Präzision Transkripte bestimmten Genen 
zuzuordnen. Zebrafische entwickeln während der ersten Wochen ihrer Ontogenese eine starke Präferenz für Artgenossen. Dies resultiert in einer Attraktion zueinander, die sich im Schwimmverhalten spiegelt - nach wenigen Wochen der Entwicklung tendieren die Fische dazu, in Richtung von Artgenossen zu schwimmen. Dies nahmen wir als Indiz dafür, dass die neuronalen Schaltkreise, die für soziale Interaktion relevant sind, sich über diesen Zeitraum hinweg entwickeln. Fische wurden für 5, 8, 14, oder 21 Tage isoliert oder in Gruppen von fünf als soziale Kontrolle gehalten. Danach wurde das Hirn extrahiert und RNA isoliert. Die Genexpression zwischen isolierten und sozialen Tieren wurde mittels Next Generation Sequencing untersucht, einer Methode, in der die Anzahl der Transkripte, die sich einem bestimmten Gen zuordnen lassen, ermittelt wird. Zu allen Zeitpunkten wurden mehr als 17.000 Gene identifiziert und in einer differenziellen Genexpressionsanalyse wurde 319 Gene gefunden, die in isolierten Tieren zu einem anderen, entweder geringeren oder höheren Grade synthetisiert werden.

Nur vier dieser Gene sind konsistent zu allen Zeitpunkten in isolierten Tieren in einem geringeren Ausmaß gefunden worden. Dabei handelt es sich um die Gene fosab, npas4a, egr1 und pth2. In der vorliegenden Arbeit fokussierte ich mich auf die Analyse von pth2, da dieses Gen zum einen stark unterrepräsentiert in der wissenschaftlichen Literatur ist und zum anderen aufgrund seiner Eigenschaften als Neuropeptid potentiell von großem Interesse sein könnte - Neuropeptide sind in vielen Verhaltensweisen relevant und können eine große Anzahl an neuronalen Zellen beeinflussen.

\section{Expressionsdynamik des Neuropeptids pth2}

Zunächst testete ich, wie genau die Expression von pth2 durch soziale Isolation bzw. ein soziales Umfeld reguliert wird. Zu diesem Zweck wurden Zebrafischlarven, die bis Tag 5 von Artgenossen isoliert hochgezogen worden waren, mit gleichaltrigen Zebrafis- 
chen in Kontakt gebracht. Dieses soziale Umfeld wurde für unterschiedliche Zeiträume aufrechterhalten, sodass ein zeitliches Profil der Genexpression erstellt werden konnte. Nach 12 Stunden sozialer Interaktion war pth2 so stark exprimiert wie in anderen Larven, die sich seit ihrer Geburt in einem sozialen Umfeld befanden. Jedoch war ein erster signifikanter Anstieg bereits nach 30 Minuten zu beobachten. In einem Experiment, in dem sozial aufgewachsene Zebrafische an Tag 5 isoliert wurden, zeigt sich, dass nach 6 Stunden sozialer Isolation bereits ein starker Abfall des Genprodukts zu beobachten war. Diese beiden Experimente zeigten, dass pth2 bidirektional durch das soziale Umfeld reguliert wird - die Expression des Gens steigt an wenn Artgenossen in der Nähe sind und sinkt ab unter Isolationsbedingungen. Desweiteren wurden Zebrafische in verschiedenen Dichten aufgezogen. Dieses experimentelle Vorgehen demonstrierte, dass pth2 umso stärker gebildet wird, je mehr Artgenossen im gleichen Tank vorhanden sind. Mit einem mathematischen Model, das sowohl die Dichte von Artgenossen als auch die Zeit, die sie miteinander verbringen, in einer linearen Regression verbindet um das Expressionslevel

von pth2 vorherzusagen, konnte gezeigt werden, dass mit diesen beiden Faktoren die Expressionsstärke des Gens sehr genau prognostiziert werden kann.

\section{Wahrnehmung von Artgenossen über das Seitenlinienorgan}

Es stellte sich anschließend die Frage, wie genau die Fische die Anwesenheit von Artgenossen bemerken - erfolgt dies über das visuelle, das olfaktorische, oder eventuell das mechanosensorische System? In einem Versuch, den visuellen Beitrag zur Regulation von pth2 zu analysieren, wurden Fische in Kammern gesetzt, die durch eine transparente Scheibe von einem angrenzenden Areal getrennt waren. Auf diese Weise konnte die Larven zwar visuellen Kontakt zu Artgenossen aufnehmen, diese aber weder chemisch wahrnehmen noch ihre Schwimmbewegungen spüren. So konnte gezeigt werden, dass 
die rein visuelle Wahrnehmung von Artgenossen nicht ausreichend ist um die Genexpression von pth2 zu steigern. Gleichermaßen konnte gezeigt werden, dass Wasser, in dem zuvor zahlreiche Artgenossen schwammen und das folge dessen mit zebrafischspezifischen Molekülen angereicht war, keinen Einfluss auf das Level von pth2 hatte. Mittels des Antibiotikums Neomycin und des Schwermetallsalzes Kupfersulfat wurde nun das Seitenlinienorgan ablatiert. Dieses Organ ist wesentlich für die Wahrnehmung von mechanischen Reizen im Wasser durch Fische. Die Behandlung durch Neomycin und Kupfersulfat zerstört die Haarsinneszellen, die verantwortlich sind für die Transduktion von Wasserwellen in neuronale Reize. In den so behandelten Fischen war es nun nicht mehr möglich, durch die Einführung von Artgenossen das Expressionslevel von pth2 zu erhöhen. Dies zeigte deutlich, dass ein intaktes Seitenlinienorgan für den beobachteten Effekt notwendig war. Nun stellte sich die Frage, wie genau der Stimulus beschaffen war, den die Fische wahrnahmen. Mit einem elektrisch steuerbaren Piezovibrator wurden verschiedene Stimulationsparadigmen versucht. Dabei wurde die Frequenz der Stimulation und die Länge der Pulse variiert. Diese Experimente führten nicht zu einem Anstieg in pth2. Nachdem das Bewegungsmuster von Zebrafischlarven genau studiert wurde, konnte ich ein Model ihrer Bewegungstendenzen erstellen, das die Dauer der Beschleunigungsphasen modellierte, in denen der Fisch mit dem Schwanz schlug und separat die Verteilung der Pausenlänge schätzte, in denen der Fisch bewegungslos war. Dieses Model wurde nun benutzt, um den Piezo zu steuern und entsprechende Vibrationen im Wasser auszulösen. Dies führte nun in der Tat zu einem Anstieg von pth2, was demonstrierte, dass die mechanische Wahrnehmung von Artgenossen nicht nur notwendig, sondern auch ausreichend für die Regulation des Neuropeptides pth2 war. 


\section{Auswirkung von pth2 auf das Verhalten von Zebrafischen}

Es ist bekannt, dass Neuropeptide für viele verschiedene Verhaltensweisen von Relevanz sind. Um festzustellen, welchen Effekt die Expression von pth2 auf Zebrafische hat, wurde im Rahmen dieser Arbeit ein erster Versuch unternommen, das Verhalten von Tieren zu charakterisieren, in denen pth2 nicht weiter funktionell war. Dazu wurden Mutanten eingeführt, in denen entweder pth2 selbst oder der entsprechende Rezeptor, pth2r trunkiert sind und denen dadurch wesentliche Elemente der normalen Sequenz fehlen. Diese Fische zeigten kein offensichtliches Defizit - ihre Schwimmgeschwindigkeit unterschied sich nicht von normalen Artgenossen, auch zeigte sich kein Unterschied in ihrer Bereitschaft, neue Areale zu erkunden, der lediglich auf die Mutation zurückgeführt werden konnte. Es wurde weiterhin untersucht, ob sich die soziale Präferenz gegenüber Artgenossen in den Mutanten anders darstellt. Zu diesem Zweck wurden die Tiere in kleine Tanksysteme eingeführt, in denen sie über transparente Zwischenwände die Möglichkeit hatten, visuellen Kontakt zu Artgenossen zu halten. In normalen Tieren führte dieser Aufbau dazu, dass ein großer Teil der Zeit direkt an der Scheibe, in kleinstmöglicher Distanz zu Artgenossen verbracht wurde. Auf dieser Ebene war kein Unterschied zu Mutanten ersichtlich. In einem weiteren Versuchsaufbau wurden die Tiere durch eine starke Vibration zu einer Fluchtbewegung veranlasst. Dieses Experiment sollte zeigen, wie nervös oder furchtsam die Mutanten im Vergleich zu normalen Wildtyptieren waren. In diesem Kontext zeigte sich ein leichter, aber signifikanter Anstieg der Fluchtreaktionen bei Tieren mit einer Mutation in pth2.

Zusammenfassend zeige ich in dieser Arbeit, dass das Neuropeptid pth2 durch die Anwesenheit von Artgenossen bidirektional und schnell in seiner Expression reguliert wird und dass diese Regulation über das Seitenlinienorgan erfolgt. Erste Versuche demonstrieren, dass pth2 eventuell dazu beiträgt, defensives Verhalten zu steuern. 


\begin{abstract}
Across the entire animal kingdom, sociality, i.e. the tendency of individual animals to form a group with conspecifics, is a common trait. Environmental changes have to be met with corresponding, quick adaptations. For social species, the presence of conspecifics is important for survival and if social animals are deprived of access to conspecifics, this can lead to strong and lasting changes on a physiological level as well as behaviour. Gene expression changes responsible for these adaptations have so far not been understood in detail. As social isolation leads to changes on a neuronal level, it is important to investigate the gene expression changes that are induced in the brain. In this thesis, next-generation RNA-sequencing was applied to zebrafish, a well-established model organism characterized by its high degree of companionship. Within the entire brain, gene expression was analysed in zebrafish that were raised either with conspecifis or in isolation, ranging from 5 to 21 days post fertilization. Using this approach, several genes were identified that were downregulated by social isolation. In this thesis, I focused on one of these consistently downregulated genes, parathyroid hormone 2 (pth2). The expression of pth2 was demonstrated to be bidirectionally regulated by the number of conspecifics present and to be responsive to changes in the social environment within 30 minutes. Regulation of pth2 does not occur by visual or chemosensory access to conspecifcs, but is mediated by mechanosensory perception of other fish via the lateral line. In an experiment using an artificial mechanical stimulation paradigm, it was shown that the features necessary to elicit pth2 transcription closely mimick the locomotion of actual zebrafish. Other, similar stimulation paradigms are not capable to induce this transcriptional response.
\end{abstract}




\section{Contents}

Zusammenfassung vii

Abstract xii

List of abbreviations xvii

1 Introduction 1

1.1 A biological account of social isolation . . . . . . . . . . . . . 1

1.1 .1 Sociality across phyla $\ldots \ldots \ldots \ldots \ldots \ldots \ldots$

1.1 .2 Behavioral and cognitive impact of social isolation. . . . . . . . 4

1.1 .3 Physiological consequences of social isolation . . . . . . . . . . 12

1.1 .4 Impact of social isolation on gene regulation . . . . . . . . . . 15

1.2 Zebrafish as a model system . . . . . . . . . . . . . . . 18

1.2 .1 Development of sensory systems in zebrafish . . . . . . . . . 19

1.2 .2 The zebrafish genome $\ldots \ldots \ldots \ldots \ldots \ldots \ldots$

1.2 .3 Social behavior in zebrafish . . . . . . . . . . . . . . 23

1.2 .4 Impact of social isolation on bony fish $\ldots \ldots \ldots \ldots \ldots$

1.3 The lateral line system . . . . . . . . . . . . . . . . . . 25

1.3 .1 Anatomy and physiology of the lateral line system . . . . . . . 26

1.3 .2 Information processing in the lateral line system . . . . . . . 26

1.3 .3 Communication via mechanosensation $\ldots \ldots \ldots \ldots . \ldots .28$

1.4 The biological relevance of parathyroid hormone-2 . . . . . . . . . . . 28

1.4 .1 Modulation of brain function by neuropeptides . . . . . . . . . . 28

1.4 .2 Class B of G-protein coupled receptors (GPCRs) . . . . . . . . . . . 29

1.4 .3 Expression patterns of PTH2 and PTH2R . . . . . . . . . 31

1.4 .4 Functions of PTH2 $\ldots \ldots \ldots \ldots \ldots \ldots \ldots$

2 Materials and Methods 36

2.1 Materials . . . . . . . . . . . . . . . . . . . . 36

2.1 .1 Chemicals \& biological material . . . . . . . . . . . . . 36

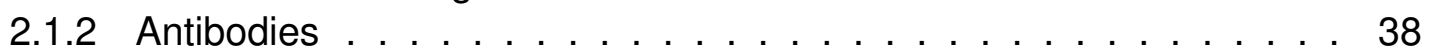


2.1 .3 Devices . . . . . . . . . . . . . . . . . . . . . . . . . . 38

2.1 .4 Solution recipes $\ldots \ldots \ldots \ldots$

2.1 .5 Animal lines . . . . . . . . . . . . . . . . . . . . . . . 40

2.2 Methods . . . . . . . . . . . . . . . . . . . . . . . . . 41

2.2 .1 Animal husbandry . . . . . . . . . . . . . . . . . 41

2.2 .2 RNA extraction . . . . . . . . . . . . . . . . . . . . . . . 41

2.2 .3 Spectrophotometric and fluorometric analysis of RNA . . . . . . . . 42

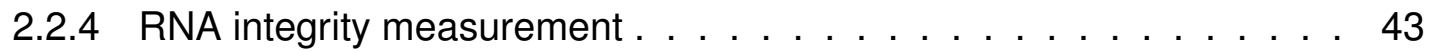

2.2 .5 Reverse transcription $\ldots \ldots \ldots \ldots \ldots \ldots . \ldots . \ldots . \ldots 44$

2.2 .6 Next generation sequencing . . . . . . . . . . . . . . . . . . . . 45

2.2 .7 Differential expression analysis . . . . . . . . . . . . . . . . 46

2.2 .8 Analysis of single-cell sequencing data sets . . . . . . . . . . 46

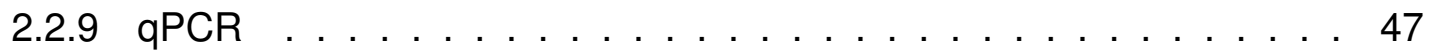

2.2 .10 Social isolation. . . . . . . . . . . . . . . . . . . 48

2.2.11 Chemosensory access to conspecifics . . . . . . . . . . . . . . 49

2.2 .12 Visual access to conspecifics . . . . . . . . . . . . . . . . . . . . 49

2.2 .13 Lateral line ablation . . . . . . . . . . . . . . . . . 50

2.2.14 Exposure to artemia and medaka . . . . . . . . . . . . 50

2.2 .15 DASPEI live staining . . . . . . . . . . . . . . . . 50

2.2.16 Extraction and modeling of behavioral features . . . . . . . 51

2.2 .17 Mechanical stimulation $\ldots \ldots \ldots \ldots \ldots \ldots \ldots$

2.2 .18 Free exploration . . . . . . . . . . . . . . . . . . 52

2.2.19 Social preference testing in the U-chamber . . . . . . . . . 52

2.2 .20 Social preference testing in the open field . . . . . . . . . . . 53

2.2 .21 gDNA extraction $\ldots \ldots \ldots \ldots \ldots \ldots$

2.2 .22 Polymerase chain reaction . . . . . . . . . . . . . . 54

2.2 .23 TA cloning . . . . . . . . . . . . . . . . . . . . . . . . 55

2.2 .24 Fixation of specimens $\ldots \ldots \ldots \ldots \ldots$

2.2 .25 In-situ hybridization . . . . . . . . . . . . . . . . . 56

2.2 .26 Immunostaining . . . . . . . . . . . . . . . . . 57

2.2 .27 Confocal imaging . . . . . . . . . . . . . . . . 58

2.2 .28 Registration to Z-Brain atlas $\ldots \ldots \ldots \ldots \ldots$

3 Results 59

3.1 Transcriptional responses to social isolation revealed by Next Generation

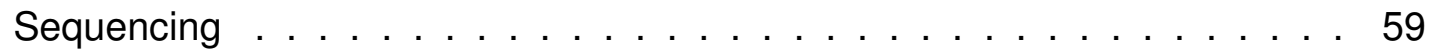

3.2 Differential expression analysis in the pth family . . . . . . . . . . . 61

3.3 Dynamics of $p t h 2$ expression $\ldots \ldots \ldots \ldots . \ldots \ldots 2$

3.3 .1 Time dependency of pth2 transcription . . . . . . . . . . . 62

3.3.2 Transcription of $p$ th2 is dependent on conspecific density. . . . . . . 64

3.3 .3 Modeling the expression landscape of $p t h 2 \ldots \ldots . . \ldots 66$

3.3.4 Dynamic regulation of pth2 occurs at all developmental stages. . . . 68 
3.4 Perception of conspecifics . . . . . . . . . . . . . . . . . . . 69

3.4.1 Chemical exposure to conspecifics does not regulate pth2 . . . . 70

3.4.2 Visual access to conspecifics is insufficient to trigger pth2 transcription 71

3.4 .3 Ablation of the lateral line with neomycin and $\mathrm{CuSO}_{4} \ldots \ldots \ldots 75$

3.4.4 Ablation of the lateral line prevents pth2 regulation by conspecifics . 76

3.4 .5 Response to the presence of different species . . . . . . . . 77

3.5 Movement parameters of isolated and social animals . . . . . . . . . . 79

3.5.1 Artificial mechanical stimulation increases $p$ th2 transcription . . . . 79

3.5 .2 Modeling larval locomotor features . . . . . . . . . . . . . . 81

3.6 Visualization of pth2 $^{+}$cells $\ldots \ldots \ldots \ldots$. . . . . . . . . . . . 84

3.6 .1 Creating an a toolset to visualize pth2 . . . . . . . . . 84

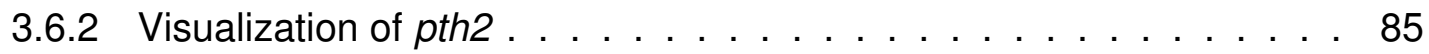

3.6.3 Registration to the zebrafish brain atlas . . . . . . . . . . 86

3.6.4 Characterization of the anatomical environment of pth2 ${ }^{+}$cells . . . . 89

3.7 Expression of $p$ th2r within the zebrafish juvenile brain $\ldots \ldots \ldots$. . . 91

3.8 Expression of pth2r in mouse and human cortical cell populations . . . . . . 92

3.9 Contribution of the stress axis to pth2 expression . . . . . . . . . . . . 95

3.10 Phenotypic analysis of loss-of-function mutants . . . . . . . . . . . . . 96

3.10 .1 Identification of loss-of-function mutants . . . . . . . . . 97

3.10 .2 Impact of $p$ th2 on locomotion . . . . . . . . . . . . . . 97

3.10.3 Degree of anxiety as measured by the startle response . . . . . . . 98

3.10.4 Impact of $p$ th2 on social preference in the U-chamber . . . . . . 100

3.10 .5 Social preference of $p t h 2$ mutants in open field . . . . . . . . 101

4 Discussion 103

4.1 Social regulation of gene expression . . . . . . . . . . . . . 103

4.2 Information processing of mechanosensory cues and transcriptional regula-

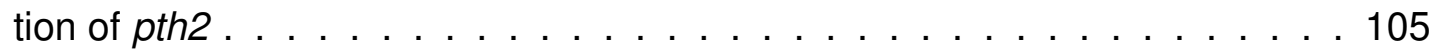

4.3 Mechanosensory perception of social cues . . . . . . . . . . 108

4.4 Modulatory effects of $p t h 2 \ldots \ldots \ldots \ldots \ldots$

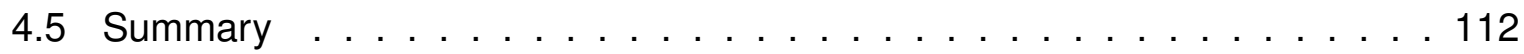

\begin{tabular}{ll}
\hline A Computer Code & 113
\end{tabular}

\begin{tabular}{ll}
\hline B Differentially Expressed Genes & 116
\end{tabular}

B.1 Differentially expressed genes at $5 \mathrm{dpf} \ldots \ldots \ldots \ldots \ldots \ldots$

B.2 Differentially expressed genes at $8 \mathrm{dpf} \ldots \ldots \ldots \ldots$. . . . . . . . . . 124

B.3 Differentially expressed genes at $14 \mathrm{dpf} \ldots \ldots \ldots \ldots$. . . . . . . 132

B.4 Differentially expressed genes at $21 \mathrm{dpf} \ldots \ldots \ldots$

\begin{tabular}{ll}
\hline$C$ List of Figures & 137
\end{tabular}

『 138 
\begin{tabular}{ll}
\hline Bibliography & 140
\end{tabular}

\begin{tabular}{ll}
\hline Curriculum Vitae & 167
\end{tabular} 


\section{List of abbreviations}

ACh acetylcholine

ACTH adrenocorticotropic hormone

AIC Akaike information criterion

ATP adenosine triphosphate

avp arginine-vasopressin

BDNF brain-derived neurotrophic factor

CDNA copy DNA

CRF corticotropin releasing factor

CAMP cyclic AMP

CREB CAMP responsive element binding protein

CGRP calcitonin-gene related-peptide

DIG digoxigenin

dpf days post fertilization

Ect ectorhinal cortex

egr1 early growth response protein 1

FISH fluorescent in situ hybridization

fosab $\mathrm{v}$-fos FBJ murine osteosarcoma viral oncogene homolog $\mathrm{Ab}$

gDNA genomic DNA 
GDP guanosine diphosphate

GPCR G protein-coupled receptor

GH growth hormone

GnRH1 gonadotropin-releasing hormone 1

GTP guanosine triphosphate

HPA axis hypothalamic-pituitary-adrenal axis

IBI inter-bout interval

IEGs immediate early genes

『L_infralimbic cortex

inf. inferior

Ins insular cortex

KO knock-out

LH $\square$ luteinizing hormone

LLC long-latency C-start

MABT maleic acid buffer with $0.1 \%$ Triton-X 100

MCH melanin-concentrating hormone

MHC major histocompatibility complex

MIP maximum-intensity projection

MON medial octavolateral nucleus 
MPL medial paralemniscal nucleus

mRNA messenger RNA

mpf months post fertilization

NGS natural goat serum

npas4a neuronal PAS domain protein 4a

nr3c1 nuclear receptor subfamily 3, group C, member 1

4-Di-2-ASP 4-(4-diethylaminostyryl)-N-methylpyridinium iodide

otpa orthopedia homeobox a

OKR optokinetic response

OMR optomotor response

oxt oxytocin

PBS phosphate-buffered saline

PBT phosphate-buffered saline with triton-X 100

PBTX phosphate-buffered saline with triton-X 100 and DMSO

PFA paraformaldehyde

PIL posterior intralaminar complex of the thalamus

PND postnatal day

pomca proopiomelanocortin a

pomcb proopiomelanocortin b 
PKA CAMP-dependent protein kinase

PrL prelimbic cortex

pthlha parathyroid hormone-like hormone a

pthIhb parathyroid hormone-like hormone b

pth1a parathyroid hormone 1a

pth1b parathyroid hormone 1b

pth2 parathyroid hormone 2

pth2ra parathyroid hormone 2 receptor a

pth2rb parathyroid hormone 2 receptor $b$

pth4 parathyroid hormone 4

PVG periventricular gray of the thalamus

PVN paraventricular nucleus

SLC short-latency C-start

SPF subparafascicular area

SPI social preference index

StAR steroidogenic acute regulatory protein

sup. superior

Znp1 synaptotagmin 1/2

TSA tyramide signal amplification 


\section{Chapter 1}

\section{Introduction}

\subsection{A biological account of social isolation}

Across all phyla of metazoans, there are examples of animals forming cohesive and distinct groups with conspecifics [1, 2, 3, 4]. This tendency has been shown to contribute to overall individual fitness by offering protection of the crowd to the individual animal [5], providing access to potential mating partners [6] and allowing for the development of elaborate social structures that enable animals to perform division-of-labor strategies or cooperative brood care [7].

Furthermore, in social species the acute or chronic deprivation of access to conspecifics induces phenotypic and physiological adaptations. These adaptations have been shown to have a net-negative long-term effect on the animal's health and survival. A wide variety of physiological systems has been demonstrated to be modulated by the social environment. For example, prolonged isolation activates the sympatho-adrenomedullary system in rats and acts as a strong stressor [8]. In some strains of mice, individual housing leads to an accelerated body weight gain and promotes the development of adiposity and diabetes [9]. As another example, a study in the fruit fly Drosophila melanogaster showed that social 
interaction drastically prolongs life expectancy in normally short-lived antioxidant enzyme $\mathrm{Cu} / \mathrm{Zn}$ superoxide dismutase mutants [10]. These findings imply that the social environment regulates physiological functions. However, there is no comprehensive understanding as to how social interaction shapes an animal on a fundamental physiological or even genetic level.

\subsubsection{Sociality across phyla}

Sociality can be thought of as the tendency of animals to congregate and live in groups, a behaviour that is referred to as gregariousness. Advanced levels of sociality include cooperation between individuals and so-called eusocial behavior as additional characteristics. Eusocial species are characterized by the presence of overlapping generations in a group of conspecifics, division of labor, and collective brood care, but not all of these features are necessarily observed together [11]. Full eusociality (i.e. the presence of all three above characteristics) is only realized in some insects (e.g. bees, termites, ants) [12], crustaceans (e.g. Synalpheus regalis) [13], rodents (naked-mole rats) [11], and humans [14]. Apart from these more sophisticated examples of sociality, congregating in groups is a prevalent mode of living for a huge number of vertebrate taxa (fig. 1.1) and is characterized by a wide range of behavioral adaptations, such as

- social eavesdropping: animals can make inferences about dominance hierarchies by observing interactions between conspecifics [15]

- social buffering: the presence of conspecifics attenuates fear responses, indicating that behavior is altered in social contexts [16, 17]

- shared decision-making: in groups of animals, decisions are made e.g. regarding the direction the group is heading into, how close the animals are to each other and 
whether a certain area is good for foraging [18]

- collective movement: if several individuals move together (e.g. schooling in fish), individual trajectories are heavily influenced by other members of the group [19]

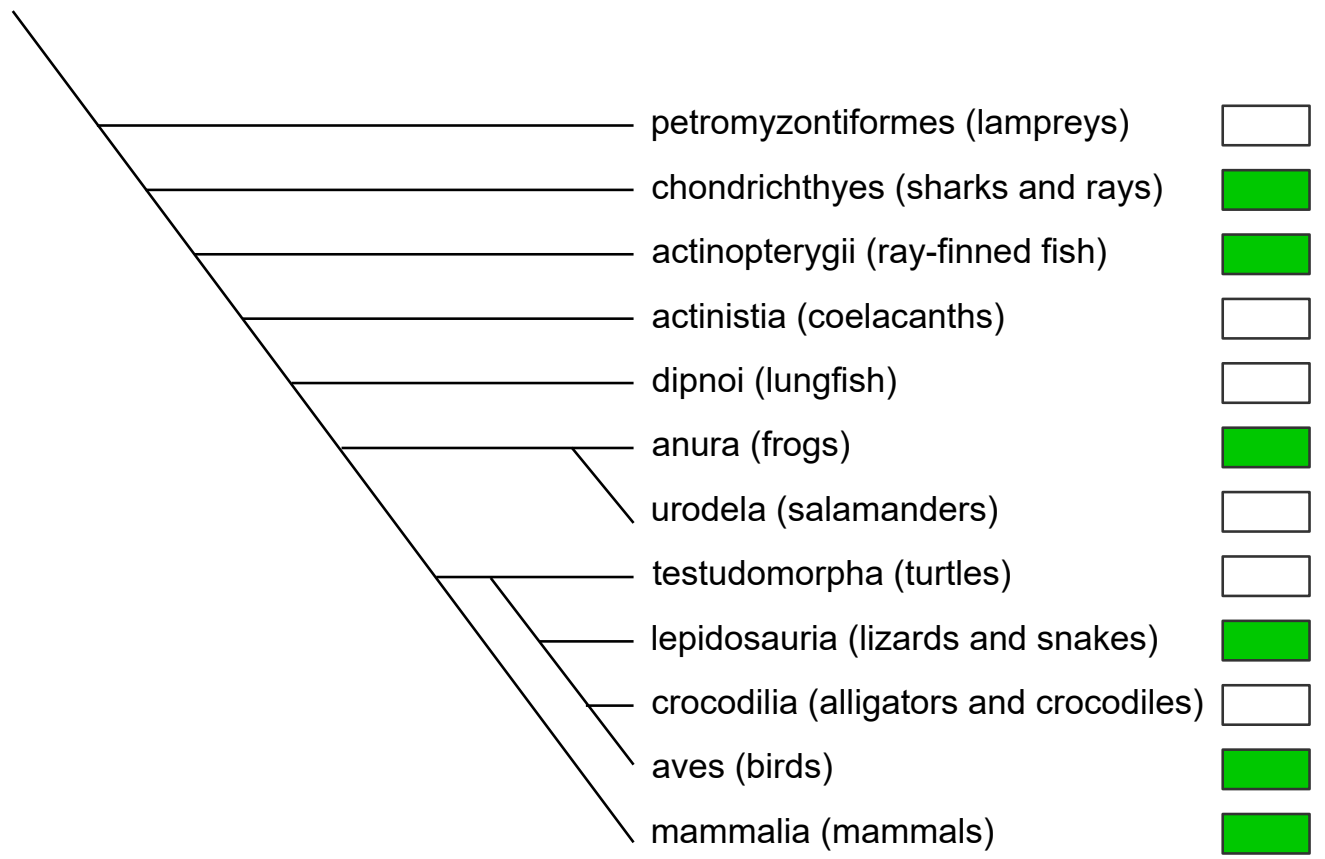

Figure 1.1: This schematic, phylogenetic tree of the vertebrates (evolutionary time is not accurately portrayed by distance) shows the groups in which living in collectives has been observed once or several times, indicated by the green boxes. Group living is thus quite prevalent already during earlier branching.

The fact that gregariousness is observed in so many species (see fig. 1.1) and that the presence of conspecifics can alter physiological processes implies an importance of group living and social interactions to an individual. Sociality opens up new possibilites to learn from and engage with the environment - in short, whether conspecifics are present or not influences the behavioral choices an animal can make. The relevance of a social environment is also reflected by findings in several species, in which larger groups are correlated with brain size. For example, locusts exist in two behavioral states, either as solitary animals when the environment provides sufficient food for all individuals, or in a 
gregarious mode when food becomes sparse [20]. A neuroanatomical comparison between solitary and gregarious locusts shows that the social animals have substantially larger brains with altered proportions [21]. Similar trend were observed in vertebrates: the highly social spotted hyena (Crocuta crocuta) has a larger neocortex than the monogamous aardwolf (Proteles cristata), the striped hyena (Hyaena hyaena), which lives solitarily or in small groups and the brown hyena (Parahyaena brunnea) which is found in groups of up to 14 individuals [22]. The same trend was observed in big cats [23], ungulates [24], and primates [25].

In most cases of group-living animals, the presence of other individuals can be considered the default mode. To better understand the importance of social interaction, it is useful to study sociality using an approach in which behavioral, physiological, and genetic adaptations are analysed in cases in which animals are deprived of social access.

\subsubsection{Behavioral and cognitive impact of social isolation}

The vast majority of studies on the effects of social isolation on animals normally living in groups has been conducted in a laboratory setting and not in the wild. Additionally, among the species that were used in experiments like this, rodents are the most-scrutinized animals, leading to a potentially biased account of the impact of social isolation. Published studies use a variety of isolation paradigms, making it more difficult to compare findings. Highlighting this points, table 1.1 lists several representative isolation studies performed on rodents over the last decades. 


\begin{tabular}{|c|c|c|c|c|}
\hline First Author & Year & Species and Procedure & Isolation paradigm & Reference \\
\hline Angulo & 1991 & $\begin{array}{l}\text { Adult male CD rats were } \\
\text { isolated for } 7 \text { - } 21 \text { days. } \\
\text { Social control: animals in } \\
\text { a group of } 3 \text {. }\end{array}$ & Not specified & [26] \\
\hline Dong & 1999 & $\begin{array}{l}\text { Male ddY mice were isolated from } \\
\text { postnatal day (PND) } 28 \text { for } 2,4,6,8 \text { or } 10 \text { weeks. } \\
\text { Social control: Groups of } 5 \text {. }\end{array}$ & Not specified & [27] \\
\hline Del-Bel & 2002 & $\begin{array}{l}\text { Male wistar rats were isolated } \\
\text { from PND } 21 \text { for } 6 \text { weeks. } \\
\text { Social control: Groups of } 6 \text {. }\end{array}$ & Not specified & 28 \\
\hline Pascual & 2006 & $\begin{array}{l}\text { Male Sprague-Dawley rats were } \\
\text { isolated from PND } 18 \text { until PND } 32 . \\
\text { Social control: Groups of } 4 .\end{array}$ & $\begin{array}{l}\text { Auditory and olfactory } \\
\text { contact }\end{array}$ & [29] \\
\hline Levine & 2007 & $\begin{array}{l}\text { Male Sprague-Dawley rats were } \\
\text { isolated from PND } 20 \text { for } 18 \text { or } 26 \text { days. } \\
\text { Social control: Groups of } 3 \text {. }\end{array}$ & Not specified & 30 \\
\hline Lukkes & 2009 & $\begin{array}{l}\text { Male Sprague-Dawley rats were } \\
\text { isolated from PND } 21 \text { for } 3 \text { weeks. } \\
\text { Social control: animals in a group of } 3 .\end{array}$ & Not specified & [31] \\
\hline Robinson & 2010 & $\begin{array}{l}\text { Male SpragueDawley rats were } \\
\text { isolated from PND } 21 \text { for } 8 \text { weeks. } \\
\text { Social control: animals in a group of } 8 .\end{array}$ & $\begin{array}{l}\text { Visual, auditory and } \\
\text { olfactory contact }\end{array}$ & 32 \\
\hline Hermes & 2011 & $\begin{array}{l}\text { Female Sprague-Dawley } \\
\text { rats were isolated from PND } \\
19-70 \text {. Social control: animals } \\
\text { in a group of } 2-3 \text {. }\end{array}$ & $\begin{array}{l}\text { Visual, auditory and } \\
\text { olfactory contact }\end{array}$ & [33] \\
\hline
\end{tabular}




\begin{tabular}{|c|c|c|c|c|}
\hline Meng & 2011 & $\begin{array}{l}\text { Male Sprague-Dawley rats were } \\
\text { isolated from PND } 21 \text { until PND } 48 \text {, } \\
\text { followed by four weeks of group housing. } \\
\text { Social control: } 4 \text { animals per cage. }\end{array}$ & Not specified & [34] \\
\hline Han & 2012 & $\begin{array}{l}\text { Male Sprague-Dawley rats were } \\
\text { isolated from PND } 21 \text { for } 2 \text { weeks. } \\
\text { Social control: animals in a group of } 3 .\end{array}$ & $\begin{array}{l}\text { Auditory and olfactory } \\
\text { contact }\end{array}$ & [35] \\
\hline Matsumoto & 2012 & $\begin{array}{l}\text { Male ICR mice were isolated at PND } \\
28 \text { for } 3,7 \text {, or } 56 \text { days. } \\
\text { Social control: } 5 \text { mice per cage. }\end{array}$ & Not specified & 36 \\
\hline Wall & 2012 & $\begin{array}{l}\text { Male and female Sprague-Dawley rats } \\
\text { were isolated from PND } 21 \text { for } 4 \text { weeks. } \\
\text { Social control: groups of } 4 \text {. }\end{array}$ & $\begin{array}{l}\text { Visual, auditory and } \\
\text { olfactory contact }\end{array}$ & 37] \\
\hline Swerdlow & 2013 & $\begin{array}{l}\text { Female and male buffalo rats were } \\
\text { isolated from PND } 24 \text { until PND } 53 \text { or } 75 . \\
\text { Social control: animals in a group of } 2 \text { - } 3 \text {. }\end{array}$ & Not specified & 38 \\
\hline Araki & 2015 & $\begin{array}{l}\text { Male ddY mice were isolated } \\
\text { from PND } 21 \text { for six weeks. } \\
\text { Social control: Groups of } 6 .\end{array}$ & $\begin{array}{l}\text { Prevention of visual } \\
\text { contact }\end{array}$ & [39] \\
\hline Kumari & 2016 & $\begin{array}{l}\text { Swiss albino female mice were isolated } \\
\text { from } 2 \text { months after birth for two months. } \\
\text { Social control: not specified. }\end{array}$ & Not specified & 40 \\
\hline Ko & 2016 & $\begin{array}{l}\text { Male and female Sprague-Dawley } \\
\text { rats were isolated from } \\
\text { PND } 21-23 \text { for } 9 \text { weeks. } \\
\text { Social control: Groups of } 2 \text { - } 3 \text {. }\end{array}$ & Not specified & 41] \\
\hline $\mathrm{Li}$ & 2016 & $\begin{array}{l}\text { Male Sprague-Dawley rats were } \\
\text { isolated from PND } 21 \text { to } 34 \text {, followed by } \\
\text { three weeks of group housing. } \\
\text { Social control: Groups of } 3 \text {. }\end{array}$ & Not specified & 42] \\
\hline
\end{tabular}




\begin{tabular}{|c|l|l|l|l|}
\hline Lander & 2017 & $\begin{array}{l}\text { Male C57BL/6 mice were } \\
\text { isolated from PND 38 for 3 weeks. } \\
\text { Social control: not specified. }\end{array}$ & Not specified \\
\hline Haj-Mirzaian & 2019 & $\begin{array}{l}\text { Male NMRI mice were isolated } \\
\text { from PND 21 - 25 to 5 weeks of social isolation. } \\
\text { Social control: Groups of } 6 .\end{array}$ & Not specified & {$[44]$} \\
\hline
\end{tabular}

Table 1.1: This table lists isolation paradigms in rodents from different studies over the past decades. The precise conditions of isolation are rarely explicitely stated (i.e. whether the animal had access to conspecifics via visual, auditory, or olfactory cues), making comparisons difficult.

However, some consistent observations have been reported in studies across phyla:

Social isolation alters defensive behavior. In some of the earliest studies on social isolation, primates were isolated some hours after birth for up to 12 months in steel cages [45]. The effect of this treatment was measured by comparing the social behavior of pairs of isolation-reared monkeys with that of pairs of socially reared animals. When first removed from social isolation, animals usually appeared unresponsive as if in shock [45]. This state was characterized by self-clutching and rocking back and forth. Afterwards, animals reared in isolation did not interact with each other except for short bursts of aggression directed towards the other monkey. The authors attributed the lack of interaction to an increased level of fear [45]. In general, when animals are paired with conspecifics after isolation, increased aggressive tendencies such as biting and chasing are common [26, 37, 46]. Beyond situations of direct social interaction, isolation increases anxiety in paradigms testing the propensity to explore novel environments: In the open field experiment, animals are placed in an empty box which they are free to roam in. Normally, no shelter or objects are provided. In these contexts, previously isolated animals consistently 
restrict their exploration to the walls of the field, avoiding the open space in which they would be more at risk of being spotted and preyed upon [33, 40, 43]. Increased anxiety levels are observed in several other experimental approaches as well: In the looming disk paradigm, a screen above the animals displays a black disk that quickly becomes larger, mimicking an attack from above. In this task, animals first freeze and then try to escape. After the disk disappers, the animals readily resume normal behavior [47]. However, animals reared in isolation show persistent freezing in this period [48]. The same tendency was observed in an auditory fear conditioning experiment. In this paradigm, a tone was paired with a footshock. Animals learned the association and after conditioning, froze in response to the tone alone. After the tone, this defensive behavior stopped and animals resumed to move around in their cage [49]. Animals reared in social isolation displayed continued freezing in this paradigm after the tone was heard [48]. Additionally, also in an unconditioned shock response experiment, in which the behavior of socially reared rats upon a footshock was compared with conspecifics reared in isolation, social isolation dramatically increased flinching and vocalizations upon a shock [50]. Attempts to attenuate these behavioral changes by resocialization have shown that increased aggression levels can be partially reversed. The success of attenuation by resocialization is negatively correlated with the length of isolation the animals previously experienced [45, 51]

This defensive behavior can be interpreted as an adaptation to social isolation. Under social conditions, animals are less at risk of being killed by predators as group living leads to a dilution of risk [52, 53] and a better chance of spotting predators [54, 55]. Indeed, it has been reported that vigilance of individual animals is negatively correlated with flock size [56].

Attention-related behaviors are modulated by social isolation. Under normal conditions, animals tune their responses to the environment they are in. For example, unan- 
ticipated noises in a quiet environment lead to a defensive reaction known as the startle response in a large number of species [57, 58, 59]. After repeated exposures to startle stimuli, animals normally habituate and display a less pronounced startle response. A similiar trend is observed if the startle stimulus is preceded by a sub-threshold stimulus (prepulse inhibition) [60,61]. In previously isolated animals, this attenuation is severely reduced: Even in a noisy environment, animals still react to sudden stimuli [38, 42]. The exact molecular mechanism underlying this adaptation is not yet clearly identified. However, some studies in rodents suggest a reduced activation of muscarinic acetylcholine (ACh) receptors in isolated animals that prevents decreased attention to sensory cues in noisy situations [62, 63].

As explained, these changes can be seen as adaptations to a situation in which the isolated animal is required to pay increased attention to its environment for prolonged periods of time in order to ensure its survival - the loss of the protective presence of conspecifics needs to be accounted for.

Learning and memory are impaired by social isolation. Additionally, deprivation of social interaction leads to a decline in working memory, extensively illustrated by work on spatial memory [64, 65, 66]. Isolated animals showed a decreased ability to identify objects whose location had changed in an object-location test [67]. In another experiment, the Morris-water-maze, animals learn the position of a hidden platform in opaque water and their ability to retain this information is later assessed by the time the animals spend in proximity of the learned position after the platform is removed. Social isolation strongly decreases the fraction of time spent in the correct quadrant in this experiment [68]. Additionally, social isolation impairs the ability of guinea pigs to learn the path through a maze, further indicating an effect of isolation on spatial learning [64]. The formation of spatial memory as described in the above examples depends in mammals on activity in the hippocampal formation. In socially isolated animals, neurotrophic factors such as 
brain-derived neurotrophic factor (BDNF) are downregulated in the hippocampus [69, 70]. BDNF normally promotes survival of neurons and has been shown to be important for long-term memory by influencing synaptogenesis [71]. In accordance with this, cells in the hippocampal formation are also morphologically altered by social isolation: A clear reduction in dendritic spine density was found in rats which were isolated for 8 weeks after weaning [72].

Another memory system that is impaired in isolated animals is social recognition. This term refers to the ability of animals to recognize specific individuals and distinguish them from others. This type of memory is necessary for the formation of social groups because it enables animals to build relationships and hierarchies [73]. Under laboratory settings, a common approach to measure the ability of e.g. a mouse to recognize a conspecific is to assess whether a decrease in spontaneous investigation behaviors are observed when the animal is reexposed to conspecific they have been introduced to before for a single session of only a couple of minutes [74]. Animals reared in isolation are capable of recognizing a familiar conspecific after a period of 30 minutes, but their recognition capabilities are decreased for longer periods (i.e. 24 hours up to 7 days) [75]. The same study demonstrated that social isolation of only one day is sufficient to prevent mice to acquire long-term social recognition memory [75]. Injection of anisomycin in the dorsal hippocampus 3 hours after the first social exposure prevented the formation of long-term social recognition memory [76]. Social isolation appears to selectively block the protein synthesis-dependent formation of long-term social recognition [75, 77, 78]. In another social recognition task, the habituation-dishabituation paradigm, this finding was corroborated. In this task, animals are repeatedly exposed to familiar conspecifics and the time they spend near those other animals normally decreases with each exposure. When a new, unfamiliar animal is then introduced, the time the experimental animal spends exploring the novel cage mate returns to its original value, confirming a stimulus-specific habituation [79]. Shahar-Gold 
et al. ([80]) extended these findings to rats and modified the habituation-dishabituation paradigm the following way: During the acquisition period animals had three consecutive 5 minute encounters with the same conspecific, separated by 10 minutes of break. After 24 hours and 7 days, the animals were tested with two additional 5 minute encounters one with the familiar conspecific and one with a novel animal. Group-housed animals were clearly able to differentiate between familar and unfamiliar conspecifics, but animals who were kept in isolation was not capable of this distinction even 24 hours after acquisition [80]. In contrast, another group of animals that was only isolated after the initial acquisition showed no deficits, suggesting that social isolation impaired memory acquisition but not retrieval [80].

In humans, social isolation has been linked with a decline of cognitive abilities [81] and several large-scale studies across different cultures and continents have shown that this finding generalizes around the globe: The US Health and Retirement Study from 1998 to 2010 showed that perceived social isolation predicted accelerated cognitive decline over 12 years independent of baseline socio-demographic factors, social network, health conditions and depression (which were assessed twice a year over the study period) [82]. The same conclusion was drawn from the China Health and Retirement Longitudinal Study earlier this year [83]. Another study on a european cohort of middle and older-aged adults showed that social isolation predicted a worse score in cognitive tests such as immediate recall, in which participants were asked to list 10 items that were just shown to them and a digit span task, which requires participants to repeat a series of digits either in the same or backward order [84].

Decreased cognitive abilities can hardly be considered a positive adaptation to social isolation and argue instead for social interaction to be a requirement for normal neurophysiological function in gregarious species. 


\subsubsection{Physiological consequences of social isolation}

If social isolation alters behavioural features, how does it affect the organism's physiology? Social isolation is generally considered to be a stressful experience and has been reported to correlate with altered activity of the hypothalamic-pituitary-adrenal axis (HPA axis). This pathway is a set of feedback connections amongst three components: the paraventricular nucleus $(\overline{P V N})$ of the hypothalamus, the anterior pituitary gland at the base of the brain, and the adrenal glands (see fig. 1.2). The PVN is located lateral to the third ventricle and contains parvocellular neurosecretory cells that project to the neurohemal median eminence, where they release corticotropin releasing factor (CRF). CRF reaches the anterior pituitary gland and stimulates there the expression and release of adrenocorticotropic hormone (ACTH), which circulates through the bloodstream and triggers the expression and release of glucocorticoids from the cortex of the adrenal gland [85]. Glucocorticoids, along with the stress response, regulate many physiological functions such as energy homeostasis, immune response, and development of the organism [85, 86]. Their effect is mediated by their binding to glucocorticoid receptors (and mineralocorticoid receptors) in the cytosol of cells. Glucocorticoid binding leads to a homodimerization of the receptors which then regulate transcription either positively by promoting gene expression as transcription factors (transactivation) in the nucleus or negatively by binding to transcription factors in the cytosol. This binding prevents translocation of those transcription factors to the nucleus (transrepression) [87]. The HPA axis is sensitive to the perception of risks and dangerous situations. Many experiments have addressed the potential regulation of effector molecules of the HPA axis by social isolation. For example, social isolation leads to increased levels of cortisol (hypercortisolism) in wild baboons [88]. The same effect is observed in other vertebrate species, including naked mole rats [89] and cichlids [90]. 
CRF and ACTH, the molecular factors that act upstream of glucocorticoid release (see fig. 1.2), could potentially mediate these changes. When rats are injected with CRF, it elevates blood plasma levels of corticosterone, the most potent glucocorticoid in rodents [91], in both group-housed and isolation-reared animals. However, the increase is more pronounced in isolated animals [92], arguing for an increased sensitivity to CRF under isolated conditions. Indeed, in rodents that have been isolated early during development, CRF receptor levels are elevated [31, 93]. Some studies in fish have shown that social isolation does not necessarily lead to an increased stress response. Giacomini et al. have demonstrated that individually housed zebrafish have a blunted cortisol response to acute stressors such as being chased for two minutes with a net [94]. In another experiment, cortisol levels were compared between socially reared adult zebrafish and conspecifics reared in isolation for six months. Between these groups, no difference in cortisol levels was found [95]. As such, it needs to be emphasized that findings regarding the impact of social isolation on HPA axis have not been consistent across studies and species, arguing for a certain context-specificity.

Several other neurophysiological features across the brain are affected by social isolation. For example, several neurotransmitters have been reported to be modulated in isolated animals, depending on the brain region. While a footshock leads to enhanced presynaptic 5-HT release in the nucleus accumbens, which is only observed in isolated rodents and not in group housed conspecifics, $5-\mathrm{HT}$ release is decreased in the prefrontal cortex and hippocampus [98]. Social isolation in rats has been shown to decrease neuronal excitability in the nucleus accumbens, which is mediated by a reduced expression of cAMP responsive element binding protein (CREB), a transcription factor. Local overexpression of CREB by injecting AAV:CREB was sufficient to partially rescue the anxiety phenotype observed after isolation [99]. Another study further revealed that the decreased overall excitability in the nucleus accumbens was mediated by increased firing rates of 
a
stress
circadian signals

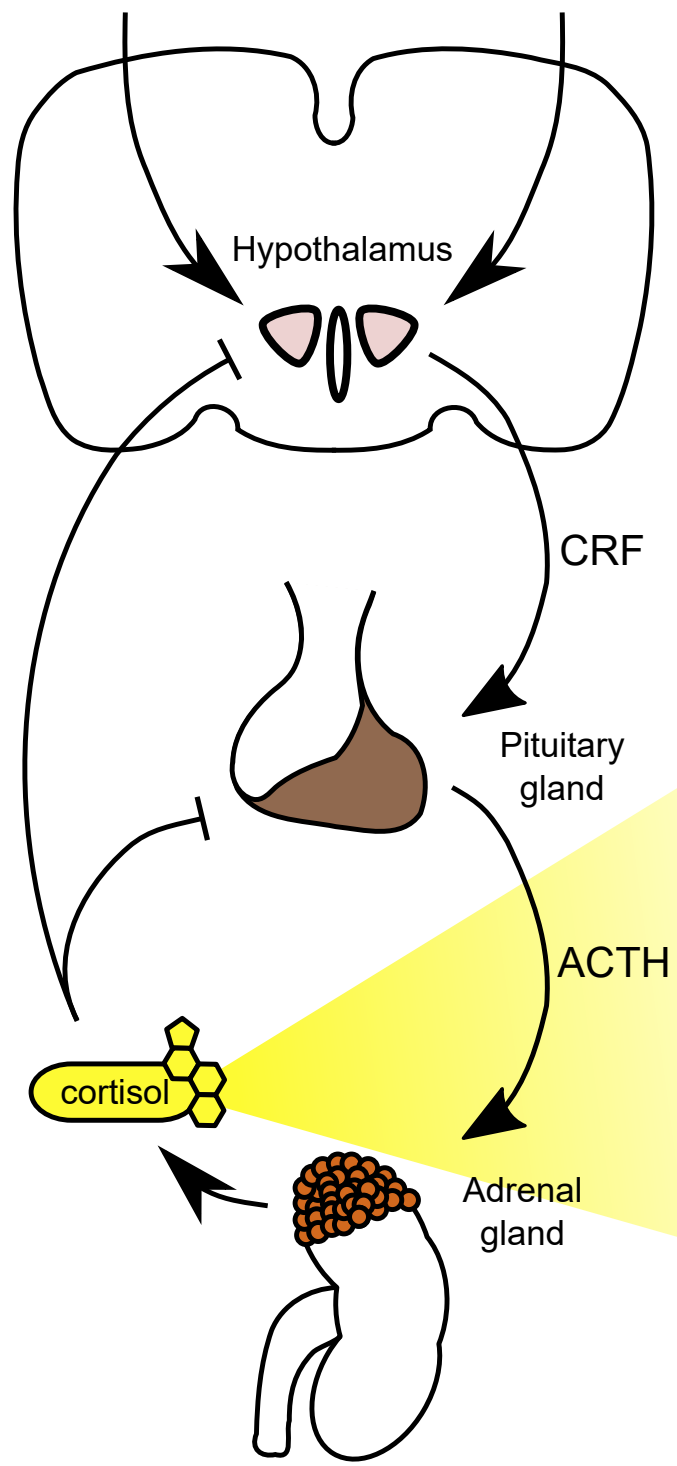

b
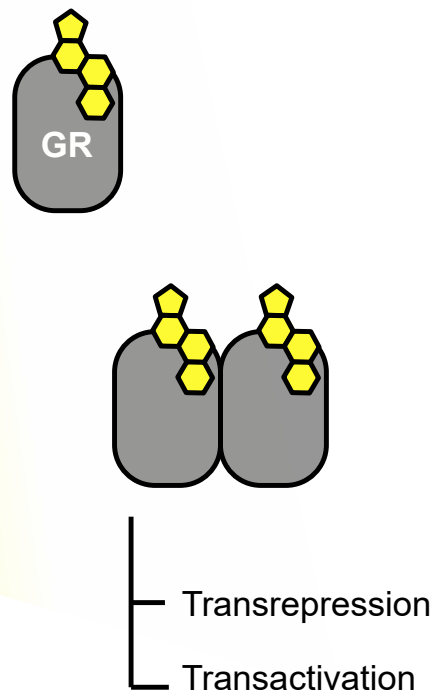

Figure 1.2: This cartoon highlights key molecules of the stress axis and the tissues involved. a, Parvocellular neurons in the $\mathrm{PVN}$ of the hypothalamus receive input about stressful situations [85]. Additionally, they are activated by circadian signals from the suprachiasmatic nucleus [96]. These cells then release CRF, which in turn triggers the relase of $\mathrm{ACTH}$ from the anterior pituitary gland [85]. Rising levels of ACTH lead to the release of glucocorticoids such as cortisol from the adrenal gland. Cortisol itself has been described to provide negative feedback to both the pituitary gland and the PVN, thus limiting furter glucocorticoid release [97]. b, Glucocorticoids are known to bind to glucocorticoid receptors (GR), inducing homodimerization [87]. The active homodimer can then either induce (transactivation) or inhibit (transrepression) gene expression [87]. 
GABAergic medium spiny neurons. This change in excitability was due to increased expression and phosphorylation of a voltage-dependent sodium channel $\left(\mathrm{Na}_{v} 1.6\right)$ [100]. In other brain areas, such as the cortex and hippocampus, $\mathrm{GABA}_{A}$ receptors are reported to be desensitized in isolated animals [101], adding further evidence that GABAergic transmission is altered by social isolation. The sensitivity of $\mathrm{GABA}_{A}$ receptors can be altered by neurosteroids such as allopregnanolone, which is synthesized in the brain of vertebrates by converting progesterone in two enzymatic steps [102]. Social isolation reduces the expression of allopregnanolone in rodents and humans [103] by downregulation of one of these enzymes, $5 \alpha$-reductase type I [101]. Supplementing socially isolated rats with allopregnanolone analogs can partially rescue the increased aggression phenotype observed in these animals [104].

Other studies found that dopaminergic neurons in the dorsal raphe nucleus are strongly excited by social cues after social isolation [105] and that basal dopamine turnover is increased by isolation in the prefrontal cortex and the amygdaloid complex [106]. By and large, several different neurophysiological systems in distinct brain areas have been found to display altered functionality in isolated animals, however, direct links with behavior have been demonstrated only in a select few cases.

\subsubsection{Impact of social isolation on gene regulation}

In his book Sociobiology, E. O. Wilson pointed out that there are recurrent organizational patterns of social life across taxa and that this is likely due to social evolution [107]. As evolution works by natural selection of genes and genetic variants, it is likely that there are specific genetic modules associated with social behavior [108]. In this framework, a comparative genetic approach studying gregarious species from different taxa might help us to understand how complex social traits derived from more ancestral conditions. As- 
sessing genes that are regulated by distinct social contexts (e.g. social isolation versus group housing) is thus a useful approach to understand how the social environment shapes an animal on a genetic level and what kind of genes are likely to play a role in social behaviour. Several studies have investigated the impact of social isolation on gene transcription and although there are still many missing pieces, several emerging themes can be identified, as described below.

Immediate-early genes are responsive to different social situations. In response to strong neuronal depolarization, a small group of genes becomes reliably upregulated without the need for prior translation of transcription factors. These immediate early genes (IEGs) are commonly used in unbiased approaches to identify neuronal correlates of particular behaviours. The differential expression of IEGs in response to social interaction or isolation has been reported and used to identify neural circuits that might be involved with social interaction [109, 110]. It is important to note, however, that the differential expression of IEGs under social isolation conditions cannot be completely explained by the presumably reduced sensory information load. This is suggested by the observed patterns of differential expression of IEGS in socially isolated animals. Indeed, several experiments showed that there is specificity in the response of IEGs to social conditions: For example, the IEG early growth response protein 1 (egr1) is downregulated by social isolation in the frontal cortex of mice, while the closely related genes egr2, egr3, and egr4 are not responsive to this condition [36]. This particular gene has been observed to be consistently downregulated in situations of social deprivation also in birds [111] and prairie voles [112] and to be highly responsive to social information in cichlids [113, 114].

Key regulators of the immune response are differentially expressed in isolated animals. About ten years ago, it was recognized that the social environment shapes gene transcription in human leukocytes [115, 116]. Social isolation downregulates the expression of genes involved with Type I interferon innate antiviral responses and in antibody 
production, but upregulates inflammation-related genes [115, 117]. These changes can be seen as adaptations to a decreased risk of socially-transmitted microbes and viruses and as preparation for fight-or-flight situations [118]. Following these initial observations in isolation-studies, a similar pattern of differential gene expression was identified in several adverse conditions (e.g. low socioeconomic status [119] or bereavement [120]), arguing for the existence of a conserved transcriptional response to adversity [117, 121] in leukocytes that is triggered by cognitive appreciation of risk or danger [118].

Hormones are regulated by social information. Another common theme of social isolation studies is the involvement of peptides with hormonal function in the response to social interaction or isolation. For example, it is well established that the nonapeptides oxytocin and vasopressin are key molecules in the regulation of social interaction [122]. It was shown that social isolation for eight weeks after weaning in rats decreased oxytocin and increased vasopressin expression levels in both male and female animals [123]. Another study showed that social isolation increased binding affinity of both neuropeptides to their respective receptors [124]. In prairie voles, chronic social isolation for four weeks reduced mRNA abundance of the oxytocin receptor in the hypothalamus [125].

An intriguing example of a neuropeptide reacting to the social environment is a member of the tachykinin family, Tac2. This peptide becomes upregulated after prolonged isolation and triggers aggressive behaviors in rodents [48]. Since a homologous tachykinin regulates aggression in fruit flies, it is conceivable that this is a conserved regulatory mechanism [126].

Another molecule, neuropeptide $\mathrm{Y}$, is an anxiolytic and neuroprotective neuropeptide [127]. It is also an important regulator of energy homeostasis and promotes food intake [128]. Exposure to stress alters its expression in different brain areas [127]. Social isolation of adult male Wistar rats for 12 weeks increased neuropeptide $\mathrm{Y}$ expression levels in the striatum and the hypothalamus [129]. In juvenile male rats, one week of social isolation 
was sufficient to increase hypothalamic expression levels of neuropeptide $Y$ [130].

Recently, the neuropeptide urotensin $2 \mathrm{~B}$ was found to be downregulated in the forebrain of songbirds after acute social isolation [131]. In songbirds, this gene is important for the development of the vocal control center in the forebrain [132].

In fruit flies, isolation-induced aggression has been shown to be mediated by another neuropeptide, Drosulfakinin [133], suggesting that neuropeptidergic control of isolationinduced brain states might be a convergent theme in social species.

Despite the insights described above, there is still no comprehensive understanding of how social isolation alters transcription. Most studies testing the genetic correlates of social interaction and isolation have focused on candidate genes. It is thus very likely that many genes involved with these factors still remain to be discovered. An unbiased approach utilizing different social species from across the phylogenetic tree would be useful in order to identify these presumed "social genes".

\subsection{Zebrafish as a model system}

Fish are a paraphyletic group of tetrapods and thus do not compose their own class (fig. 1.1. They branched from other tetrapods about 360 million years ago and extant species make up about $50 \%$ of all known vertebrates [134]. These animals display a surprisingly huge variety of social systems, including solitary lifestyle, monogamous mating partners as well as harems [134] and tremendously huge schools of fish that consist of up to 3 billion individuals [135, 136]. Zebrafish are one of the species that are routinely worked with in biomedical research, mainly due to their short generation time, their translucency during the larval stage and their well annotated genome, which makes the animal amenable to genetic manipulation and analysis. In the context of social interactions and isolation studies, it is of particular relevance that zebrafish, due to their size, can easily be housed in 
large numbers without requiring a lot of space. From an early developmental stage, these animals are attracted to conspecifics [137, 138] and at around four weeks of age display a strong and stable preference to stay close to other zebrafish [139]. This characteristic feature enables them to form cohesive groups that move together, normally referred to as schools [140].

\subsubsection{Development of sensory systems in zebrafish}

Zebrafish mate by external fertilization, usually resulting in several hundreds of eggs per clutch. Embryogenesis occurs over the course of only a few days (see figure 1.3), resulting in a fully independent larva at about 5 days post fertilization (dpf). Upon reaching the juvenile stage with 30 days, animals develop an adult pigmentation pattern and fins. Sexual maturity is usually reached within $90 \mathrm{dpf}$. Zebrafish can reach up to 4 years of age but start to lose fecundity and show other signs of age-related decline at 1 - 2 years of age under normal laboratory settings [141]. Zebrafish develop the capacity to perceive their surroundings and act on this information quite early. Already between 2 and $4 \mathrm{dpf}$, animals react to sudden light intensity changes with a startle response and track the stripes on a rotating drum beginning around $3 \mathrm{dpf}$, which is evidence for pattern vision [142]. This ability to recognize moving visual patterns has been investigated by utilizing two behavioral paradigms, the optokinetic response (OKR) and the optomotor response (OMR). The OKR are stereotypical eye movements, saccades, that track rotating or moving stripes. For this paradigm, the underlying circuit has been described in detail. 


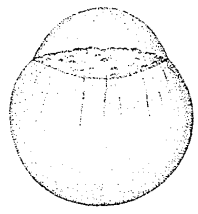

1-cell $0.2 \mathrm{~h}$

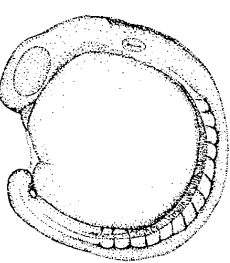

14-somite

$$
16 \mathrm{~h}
$$
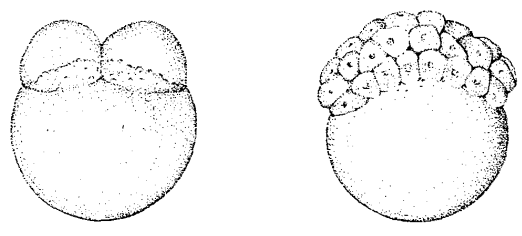

64-cell

$2 \mathrm{~h}$

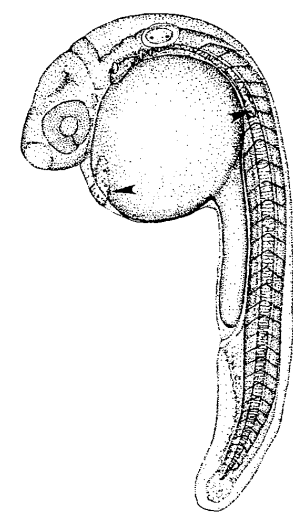

prim-6

$25 \mathrm{~h}$

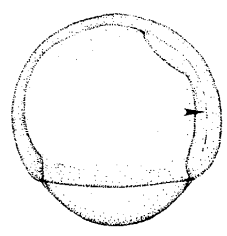

$75 \%$-epiboly

$8 \mathrm{~h}$

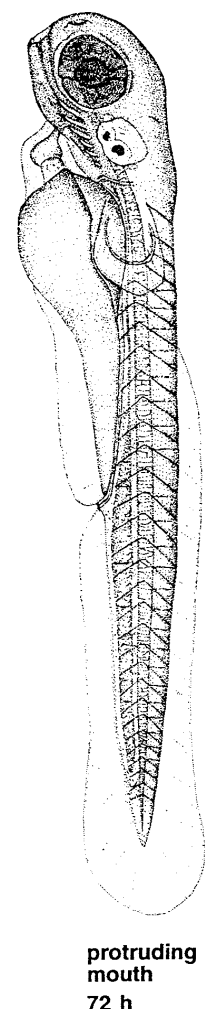

Figure 1.3: Here, the first stages of zebrafish development are shown. Early embryogenesis occurs comparably fast and with 3 days of age, zebrafish hatch. They become entirely independent shortly after. The stages depicted are taken from Kimmel et al., 1995 [143], and were reused with kind permission of Wiley (@1995 WILEY-LISS, INC.).

The velocity-to-position integrator that relates eye position to the velocity of the moving field is a group of hindbrain neurons that encode eye position via a population code of persistent activity [144] and drive oculomotor nuclei [145]. When moving stripe patterns are projected on the ground of a tank, zebrafish tend to swim in the direction opposite 
to the optic flow. This behavior is robustly observed at $7 \mathrm{dpf}[146]$. On a sensory level, this is mediated by direction-selective retinal ganglion cells [145]. Signals from both eyes are integrated in the pretectum [147] and this information is used in the hindbrain reticular formation to induce forward and turning motion by activating spinal cord neurons [148]. When larval zebrafish are given the choice to freely explore their surroundings, they tend to move towards better illuminated areas (positive phototaxis). Whole-brain imaging of restrained animals in a virtual environment showed that a self-oscillating population of hindbrain neurons in rhombomer 2 and 3 controls the direction of swim bouts and biases them towards better illuminated areas [149, 150]. Zebrafish are able to change the distribution of melanin pigments in their skin to match ambient illumination levels, which is mediated by a direct projection from the retina to the hypothalamus, where melanin-concentrating hormone $(\mathrm{MCH})$ is released, regulating dispersal of pigments [151]. Animals with visual impairments are unable to utilize this system and appear darker than healthy fish already at early larval stages [152]. Furthermore, the ability to effectively catch prey is also mediated primarily by vision and is observed as early as at 4 dpf [153]. In the context of social interaction, the reinforcing effect of visual access to conspecifics has been described as early as 6 dpf [137]. Over development, the visually mediated attraction to conspecifics becomes increasingly stronger and reaches adult levels in juvenile animals at around 28 dpf][139].

The functionality of chemosensation has been demonstrated at early developmental stages in zebrafish. At 3 dpf, zebrafish are repelled by chemical irritants [154, 155], evidence of their capacity for chemosensation. With $4 \mathrm{dpf}$ larvae increase their swimming speed in response to the presence of amino acid chemostimulants in the surrounding medium [156]. Pairing natural odorants such as the amino acids alanine and valine as well as artificial odorants such as phenylethyl alcohol with food flakes as unconditioned simulus increased appetitive swimming, measured as the number of turns of more than 
90 degrees over a 30 seconds period [157]. This effect was not observed when both nostrils were closed, demonstrating that the conditioning is based on olfactory cues [157]. Chemosensation of social cues has been demonstrated as well. Juvenile zebrafish are able to distinguish between unfamiliar kin and non-kin conspecifics based on olfactory stimuli [158]. This ability is acquired during an imprinting phase on day 6 post fertilization [159]. It was later shown that the process involves recognition of the major histocompatibility com-

plex (MHC) class II [160], which is a specific task of crypt cells in the olfactory epithelium of zebrafish [161]. The presence of chemical cues from conspecifics in the holding water was shown to lead to alterations in swimming behavior of adult zebrafish, although the exact identity of those cues was not elucidated [162].

The spatial organization of the olfactory system in zebrafish is similar to mammals, although the number of odorant receptor genes existent in zebrafish and the number of glomeruli is about one order of magnitude less than what is observed in mammals [163].

Another important sensory system, the lateral line, will be discussed in a separate section.

\subsubsection{The zebrafish genome}

The zebrafish genome contains more than 26,000 annotated genes on 25 chromosomes. This high number is due to the genome duplication that occurred after the split between fish and other tetrapods, leading to a huge number of duplicate genes in fish which allowed for a high rate of genetic diversification [164]. Nevertheless, when comparing human and zebrafish genome, about $47 \%$ of all human genes have a one-to-one relationship with an ortholog in fish and $71 \%$ of human genes have at least one orthologous gene in zebrafish [164]. A first attempt to functionally assess the zebrafish genome and make this species amenable to genetic analysis was performed in the 1990s in the laboratory of 
Christiane Nüsslein-Volhard, when her group conducted a mutagenesis screen and identified about 4,000 mutant lines that showed abnormal behavioral or developmental features [165]. By now, the zebrafish has been genetically modified by TALENs, Tol2-mediated transgenesis and CRISPR-based approaches [166, 167]. In addition, a large number of cell-type specific driver lines on the basis of the Gal4/UAS system have been established. Building on this molecular toolkit, an increasing number of behaviors has been linked to underlying cellular and molecular correlates [147, 149].

\subsubsection{Social behavior in zebrafish}

Zebrafish are group-living animals that display a wide variety of social behaviours. On the most basic level, a strong tendency to congregate with conspecifics is observed in juvenile and adult zebrafish. This social preference increases with development and matures at about $28 \mathrm{dpf}[139]$. The sight of conspecifics has been linked to a specific dopaminergic reward system in adults [168], but rewarding properties of visual access to conspecifics have been demonstrated as early as at $6 \mathrm{dpf}[139,137]$, arguing that social access and interaction are of importance already at the larval stage. Indeed, the presence of conspecifics shapes behavioral features of larval zebrafish [169] and computational modeling has demonstrated that the trajectories of $7 \mathrm{dpf}$ larvae are already biased towards the location of conspecifics, a tendency that grows stronger with age [138, 139]. In adult fish, this preference in conjunction with the ability to adjust one's own movement with the locomotion of close neighbors enables complex collective behaviors such as schooling and shoaling [140]. These behaviours are defined as distinct modes of collective behavior in older juvenile and adult fish. Their characteristic difference is the polarity of the group: It was found that the animals always follow the attraction rule outlined above [138, 139], thus forming a cohesive group. However, they can either swim in a polarized manner in the same di- 
rection (schooling) or be more dispersed, thus resulting in less directed movement and more exploration of the current location (shoaling) [170]. This distinction was first made in zebrafish in 2012, when Miller and Gerlai plotted the distribution of the polarization of a group of fish and showed its bimodality, corresponding to the two modes described above [140]. Schooling and shoaling have been described as a defensive mode used to protect the group against predators [171].

Social conditions also shape behavioral responses to threats. When zebrafish are confronted with a substance called Schreckstoff (literally translated to fear stuff), which is released by conspecifics when they are hurt, they enter a state of intense distress, indicated by prolonged freezing and strongly increased swimming speed [172]. Visual access to conspecifics not exposed to Schreckstoff can significantly decrease the effect of this compound, a phenomenon referred to as social buffering [17]. This indicates that access to other fish modulates the neural circuitry involved with assessing dangerous situations and adapting the behavioural response according to the state of surrounding fish.

Zebrafish have been shown to pay detailed attention to social interactions in their surroundings. For example, social information is gauged from observing conspecific interaction (social eavesdropping), providing an animal with information about its place in a hierarchy. This behavior has been linked to profound changes in the brain transcriptome of adult zebrafish [173, 15]. These findings highlight that zebrafish display a variety of social interactions and utilize social information for decision-making.

In other fish species, too, social information has been shown to be relevant for the animal's behavior. Knowledge about the social hierarchy is important for social plasticity, a phenomenon in which animals adapt their behavior according to their status within a group [174, 175]. Socially dominant fish are characterized by their ability to win fights in diadic, aggressive encounters. Dominant males have been shown to display territorial behavior [176] and in both sexes, dominance is associated with higher reproductive success [177]. 


\subsubsection{Impact of social isolation on bony fish}

How are fish affected when they are deprived of access to conspecifics? A consistent finding is a positive association between isolation and aggressive behavior, an effect observed in cichlids [178, 179], the fighting fish Betta splendens [180] and the swordtail Xiphophorus helleri [181]. Another effect is the impairment of neurogenesis in isolated fish, supported by findings in trout [182], electric fish [183], and zebrafish [95]. In contrast with the results discussed in section 1.1.2, social isolation led to increased locomotion and decreased anxiety levels in adult zebrafish [184, 185, 110]. However, it induced decreased locomotion in larvae [186], indicating a developmental stage-dependent effect. Overall, these findings indicate that social isolation in fish affects systems similar to what was observed in other species, although a detailed understanding ranging from behavioral to the molecular level is certainly missing.

\subsection{The lateral line system}

In the present study, mechanosensation was identified as an important channel for social information (further discussed in the results section). How are mechanical cues perceived in water? When animals move in water, they induce pressure gradients around them. A sensory system tuned to these kind of mechanosensory stimulation would be highly informative for an animal. Indeed, all fish and a majority of aquatic vertebrates possess a so-called lateral line system enabling them to detect movement-induced waves or vibrations in their proximity [187]. Initially believed to deliver only coarse mechanosensory information, recent research has demonstrated that perception via the lateral line can be used in complex interactions, such as social communication [188]. 


\subsubsection{Anatomy and physiology of the lateral line system}

The lateral line consists of individual sensory units, neuromasts, that are distributed along the body of the animal in two major systems: the anterior lateral line, covering mainly the head of the animal, and the posterior lateral line localized caudally. These neuromasts contain specialized epithelial cells with apical bundles of stereocilia that are embedded in a gelatinous cupula [189]. Upon deflection, mechanosensitive ion-channels in the membrane of the stereocilia open up and allow for the influx of $\mathrm{K}^{+}$and $\mathrm{Ca}^{2+}$. Hair cells possess a membrane potential $\left(V_{m}\right)$ of approximately -70 to $-50 \mathrm{mV}$ [189, 97]. The extracellular $K^{+}$concentration is about $2-4 \mathrm{mM}$ and the intracellular one reaches $125-$ $140 \mathrm{mM}$ [189], resulting in an inward driving force tha pulls $K^{+}$into the cell upon increased conductance. A moderate deflection of the hair cell bundle thus depolarizes the membrane potential whereas movement in the opposite direction actually slightly hyperpolarizes the cell. This is due to the fact that the mechanosensitive channels are positioned in a way that movement in one direction stretches them while they are actively closed by movement of the hair bundle in the opposite direction, increasing the dynamic range over which a hair cell can encode information [190]. Depolarization increases glutamate release at the basal part of the hair cells, which induces graded potentials in the afferent fibers conveying this information to downstream areas of the lateral line system [189].

\subsubsection{Information processing in the lateral line system}

The first step of signal filtering occurs at the level of neuromasts, which are arranged in two locations: the superficial ones, that are tuned to frequencies below $30 \mathrm{~Hz}$ and canal neuromasts, that are receptive from $30-200 \mathrm{~Hz}$ [191]. During locomotion, efference copies of the motor signal inhibit neuromast activity [192]. Information from the lateral line is then conveyed via afferent fibers to the ganglia of the anterior and posterior lateral 
line, respectively [193]. At this point and also in more central processing centers, projections adhere to a somatotopic mapping principle [194, 195]. The next relay station is the medial octavolateral nucleus (MON). Second-order projections from the MON terminate both ipsi- and contralaterally in the torus semicircularis (see figure 1.4). Finally, information is projected to different diencephalic nuclei. Dorsal parts of the telencephalon have also been described to receive lateral line input [196]. Electrophysiological recordings have demonstrated that units in higher-order brain areas involved with the processing of information from the lateral line become tuned to increasingly complex patterns. For example, medullary and midbrain lateral line units react to amplitude-modulated sinusoidal water motions [197] and units in the torus semicircularis have been shown to be phase-locked to particular frequencies [198]. Although there is ample evidence to support the notion that fish use lateral line information to hunt and navigate their environment, it is not clear yet how complex the representations are that are built with these sensory perceptions [187].

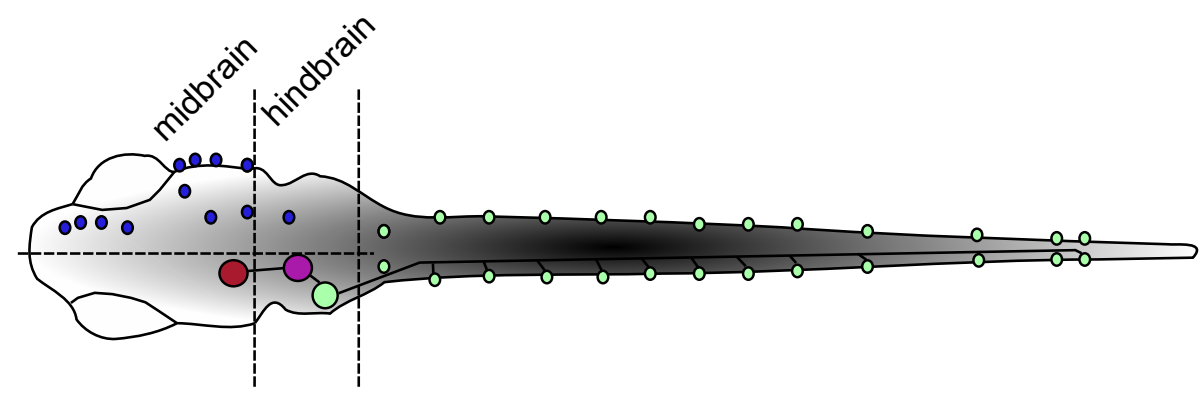

anterior lateral line

posterior lateral line

Figure 1.4: This cartoon depicts the position of individual neuromasts of the anterior lateral line as blue and those of the posterior lateral line as green dots. Higher-order processing stations are for reasons of clarity only shown for one side of the posterior lateral line, however, the other side as well as the anterior lateral line are following basically the same pathway. Afferent fibers convey signals from the neuromasts to the corresponding lateral line ganglion (here shown in the same color as the posterior neuromasts, but with a bigger radius) [193]. From there, signals are transferred within the hindbrain to the MON (shown in purple). This nucleus projects then to the torus semicircularis within the midbrain (shown in red) [196]. The MON and the torus semicircularis are the best-described higher-order processing centers of lateral line information. However, projections to other areas such as the telencephalon and the thalamus have been described as well (see section 1.3.2. 


\subsubsection{Communication via mechanosensation}

Only a few studies have focused on higher-order perception via the lateral line. Butler and Maruska showed that the ablation of the lateral line increases the propensity of cichlids to engage in a fight, presumably because they utilize lateral line information to gauge the size of their opponent [188]. It was also demonstrated that fish use vibrational cues to elicit spawning behavior during mating [199]. These results indicate that mechanosensory perception via the lateral line might indeed be an important channel for communication or gathering social information from the environment.

\subsection{The biological relevance of parathyroid hormone-2}

In the experiments discussed in this thesis, the expression of the neuropeptide parathyroid hormone 2 (pth2) was found to be regulated by the social environment. What is known about this molecule and its function?

\subsubsection{Modulation of brain function by neuropeptides}

Neurons propagate information on different timescales: activation of ionotropic receptors and ion channels rapidly increases conductance for the corresponding ions, which can result in changes in the cell's membrane potential [200]. These depolarizations can induce action potentials that are quickly transmitted over long distances [201]. On another level, network properties are also modulated by the presence of many small peptides, typically acting on a G protein-coupled receptor (GPCR). These neuromodulators can alter the in-

trinsic firing properties of neurons and change effective synaptic strength. This indirectly reconfigures neuronal circuits on top of the connectome by strengthening some synapses and weakening others, potentially strongly altering network computations [202, 203, 204]. 
Neuropeptides are genetically encoded as prepropeptides that become active by proteasemediated cleavage [205]. They constitute a diverse class of signaling molecules and 30 different neuropeptide families have been traced back to the last common ancestor of protostomes and deuterostomes, indicating that neuropeptidergic signaling is an ancient and conserved mode of neuronal communication [206, 204, 207]. Neuropeptidergic modulation can exert influence by very different means and either change or help to conserve functionality over a wide variety of systems. For example, changes in ambient temperature are a relevant factor that can influence neuronal excitability in poikilotherm animals. Work on the stomatogastric ganglion in crustaceans has demonstrated that several neuropeptides increase robustness of neuronal timing despite changes in temperature, thus enabling a neural circuit to serve its function in different environmental conditions [208, 209]. Next to maintaining functionality by promoting network robustness, neuropeptides can also change behavioural outputs of neuronal circuits. One example of this feature is a study in the nematode Caenorhabditis elegans which demonstrated that nociceptive inputs promote the aggregation of these normally solitary worms via the neuropeptide npr-1 [210]. In general, several hundreds of neuropeptides have been described in metazoans and only some of them have been assigned a function [206]. The neuropeptide pth2, which this work focuses on, belongs to a certain class of neuropeptides which has been characterized to some extent.

\subsubsection{Class B of G-protein coupled receptors (GPCRs)}

Molecules activating GPCRs are normally classified based on the similarity of the receptors they activate. All GPCRs contain 7 transmembrane segments and share a particular signaling mechanism, which is their interaction with G-proteins, heterotrimeric GTPases. It is currently believed that all GPCRs share a common evolutionary ancestry [211]. 
The neuropeptide pth2 activates a receptor which is part of the family-B1 GPCRs [212]. Members of the B1 subfamily are classical hormone receptors with ligands of $27-141$ amino acid residues. All members of this subfamily have been demonstrated to regulate intracellular cyclic AMP (CAMP) concentrations by activating adenylate cyclase through the stimulatory $\mathrm{G}$ protein $G_{S}$ [212] (see fig. 1.5). However, the different receptors have been implicated in very different physiological functions, such as glucose homeostasis by the PAC1 receptor [213], release of ACTH by the CRF1R [214], and calcium homeostasis by the PTHR [215].

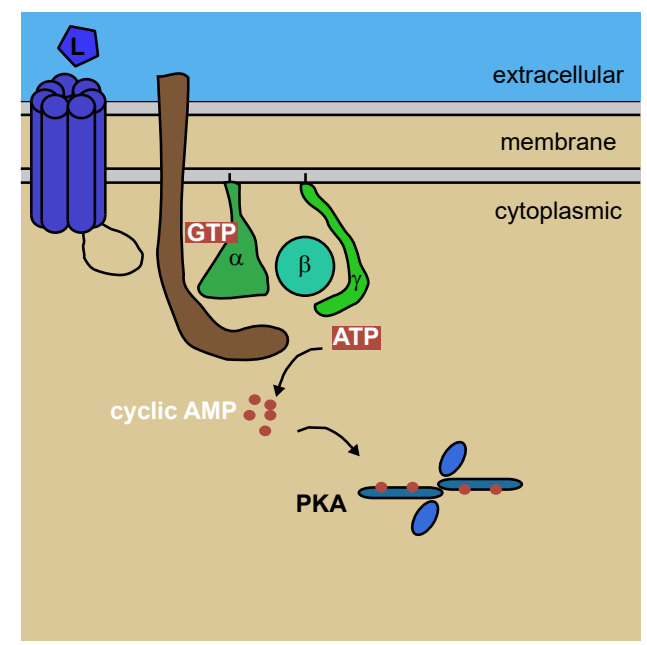

Figure 1.5: This cartoon illustrates the signaling cascade described for stimulatory GPCRs $\left(\mathrm{G}_{S}\right)$, which are described for all members of the class B of GPCRs. Binding of the ligand $\mathbf{L}$ activates $G_{S}$ which promotes the exchange of a bound guanosine diphosphate (GDP) to a guanosine triphosphate (GTP). This leads to a conformational change of the G-protein, after which it activates the integral membrane protein adenylyl cyclase (shown in brown). Adenylyl cyclase now begins to catalyze the conversion of adenosine triphosphate (ATP) to CAMP. The secondary messenger targets and activates in most cells cAMP-dependent protein kinase (PKA), a heterotetrameric enzyme which is normally inhibited by the presence of a dimer of regulatory subunits. CAMP binds and inhibits the regulatory subunits, which allows the catalytic subunits to become active and phosphorylate target proteins. Among the many demonstrated effects of this increased phosphorylation is differential gene expression via the activation of enhancers and the modulation of potassium channel permeability [212, 216]. 


\subsubsection{Expression patterns of PTH2 and PTH2R}

Pth2 was first been characterized in bovine hypothalamic extracts by Ted Usdin in 1997 [217]. Initially referred to as tuberoinfundibular peptide of 39 amino acids length (TIP39), pth2 has been identified in several tissues: in the brain and testis [218], kidney and pancreas [219], and heart in rats [220]. A more in-depth characterization has been performed on brain cell groups that express pth2, identifying two restricted localizations in rodents and macaque (see fig. 1.6):

- the periventricular gray of the thalamus (PVG)

- the posterior intralaminar complex of the thalamus (PIL)

- the medial paralemniscal nucleus (MPL)

Both $\mathrm{PVG}$ and $\mathrm{PIL}$ are located in the subparafascicular area (SPF) of the posterior diencephalon and were initially believed to contain a contiguous group of pth2 ${ }^{+}$cells [218]. However, subsequently, the SPF was separated into the magnocellular or medial subparafascicular nucleus (containing the $\mathrm{PVG}$ ) and the parvicellular or lateral subparafascicular nucleus (containing the $\mathrm{PIL}$ ). With 600 - 1,000 cells per hemisphere, the PVG contains the largest group of $p t h 2^{+}$cells in the brain of young rodents [221, 222]. These neurons roughly overlap with the position of the A11 dopaminergic cell group, but previous studies were unable to identify pth2/tyrosine hydroxylase double-labeled cells, suggesting that these cell types are distinct [221, 223].

The lateral thalamus contains roughly 200-300 small pth2 $2^{+}$cells in the PIL.They are located medial to a cluster of calcitonin-gene related-peptide (CGRP) expressing neurons and a fraction of the cells is double labeled with both CGRP and pth2 [221]. The neurons in the medial part of the SPF express c-fos after male ejaculation, whereas the lateral cells do not, supporting the distinction between these cell groups [224]. PVG and PIL 
receive neuronal input from very similar brain areas. Forebrain regions, such as the medial prefrontal, insular, and entorhinal cortices, the subiculum, amygdala and the lateral septum have been shown to project both diencephalic pth2 ${ }^{+}$cell groups [225]. The auditory cortex projects only to the $\mathrm{PIL}$ [226, 227]. A large part of diencephalic projections arise from hypothalamic regions, the preoptic area, the anteroventral periventricular nucleus, and the dorsal premamillary nuclei. Deep layers of the superior colliculus and cortical areas of the inferior colliculus are labeled by retrograde tracers [228].

Medially positioned to the fibers of the lateral lemniscus, there is another distinct pth2 ${ }^{+}$ group of cells located in the rostral pons [218]. These cells receive rich input from brain areas involved with the processing of sensory information, such as the auditory cortex, and both the superior and inferior colliculus as well as the lateral preoptic area, the $\mathbb{P I L}$ and hypothalamic regions [229].

All three groups of $p t h 2^{+}$cells have widespread projections. After electrolytic lesion of the PVG, a decreased number of pth2 ${ }^{+}$projections was detected in the medial prefrontal cortex, the lateral septum, the amygdala, the thalamic paraventricular nucleus, the hypothalamus and the periaqueductal gray, indicating that these regions receive input from the PVG [221]. Neurons in the PIL project to the ipsilateral amygdala and hypothalamus. Few projections were found to reach other forebrain structures such as the prefrontal cortex and the nucleus accumbens [221]. From the MPL, pth2 $2^{+}$projections reach the superior and inferior colliculus and the spinal cord. However, these data relied on lesion studies, anterograde tracings from the MPL have not been successful, leaving many of the MPL projections to still be verified [221, 225].

The pth2 receptor is abundantly expressed in the brain of rodents, macaque, and fish [230, 231, 222, 232], with the most detailed description being available for rodents. In general, the regions expressing pth2r also receive projections from one of the $p t h 2^{+}$clusters. Particularly high concentrations of the receptor were found in the lateral septum and the 
amygdala, the preoptic area and the hypothalamus [225]. In fish, the receptor is found to be strongly expressed throughout the entire brain [232].

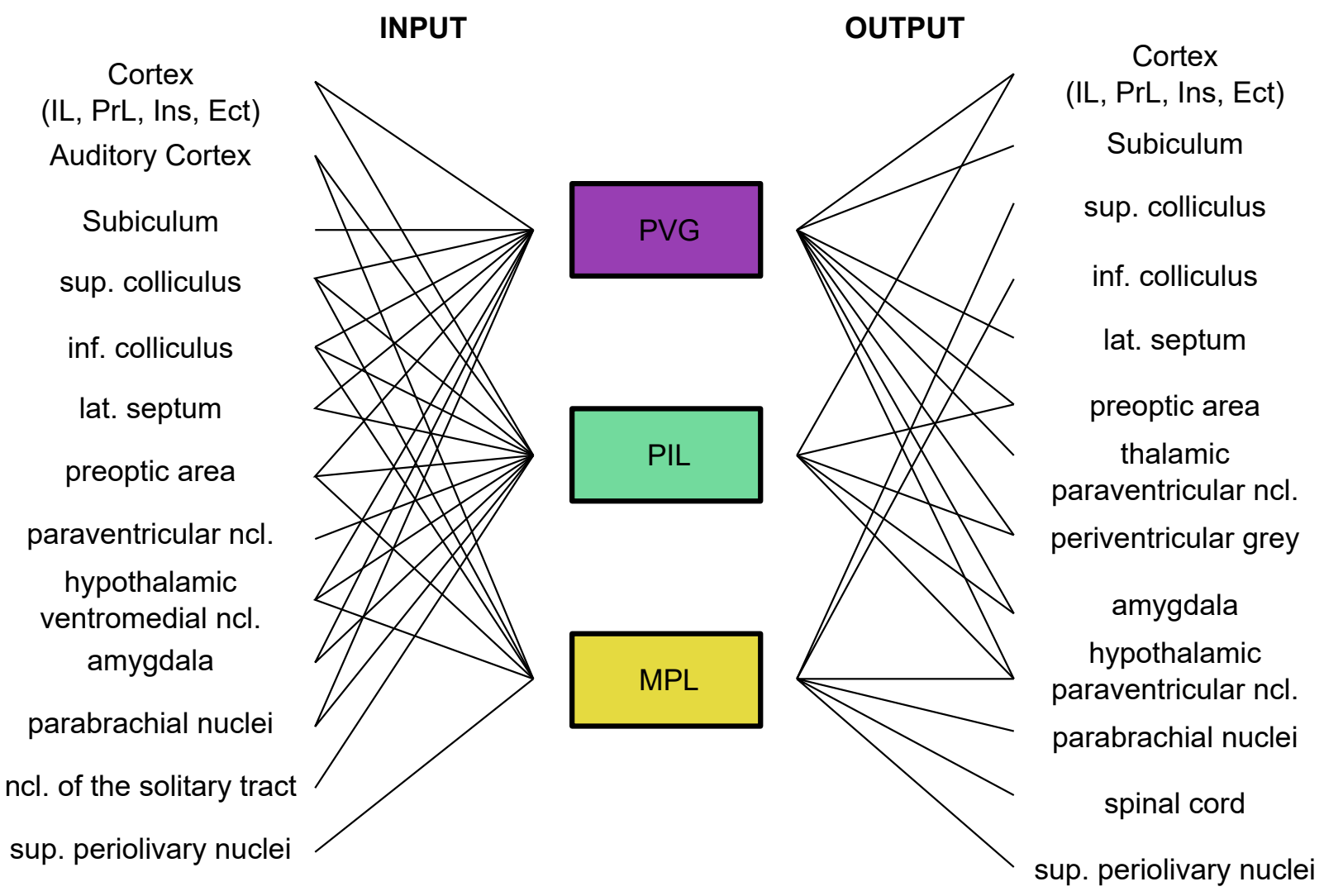

Figure 1.6: Projections to the three known locations of pth2+ cells in rodents are shown on the left, whereas the sites these cells project to are shown on the right. Abbreviations: infralimbic cortex (IL), prelimbic cortex (PrL), insular cortex (Ins), ectorhinal cortex (Ect), superior (sup.), inferior (inf.). Adapted after Dobolyi et al. 2010 [225].

\subsubsection{Functions of PTH2}

Owing to the multitude of projections pth2 ${ }^{+}$cells make, it is not easy to pinpoint a particular function for this neuromodulator system. However, in rodents it has been implicated in several processes. The injection of PTH2 in the spinal canal leads to a dose-dependent nocifensive response, scratching and licking [218]. Furthermore, it decreased latencies to 
withdrawal in paw pressure experiments. If PTH2 was injected together with a sequestering antibody against it, pressure sensitivity decreased again [218]. The pth2 receptor is expressed in lamina II of the spinal cord, where most nociceptive afferents terminate. These results suggest that pth2 plays a role in the modulation of pain perception.

As several regions in which pth2+ fibers are found are thought to be important for anxiety and depression-like behavior, the effect of pth2 was analysed after its administration in rats and by observing behavior in knock-out (KO) mice [233, 234]. In general, [KO animals could not be distinguished from wildtype littermates; no strong phenotypic abnormalities are detected. In open field explorations, no evaluated behavior was altered [234]. In a different set of experiments testing anxiety in the elevated plus maze, rats entered the open arms more often after administration of PTH2, suggesting an anxiolytic effect [233]. However, KO mice did not display any strong and reliable phenotype in any of the paradigms tested, including a novel open field, dark-light emergence or the elevated plus maze [234]. Differences between the KO and WT mice became apparent when animals were tested under stressful conditions, such as after short restraint or under a bright light source. In these cases, $\mathrm{KO}$ mice visited the open arms fewer times and also spent less time there [234]. In experiments in which the animals underwent fear conditioning, $\mathrm{KO}$ animals froze significantly more than their wildtype littermates when the shock-paired tone was heard 24 hours after the training session [234].

Other datasets suggest that pth2 is involved with neuroendocrinergic regulation. After intracerebroventricular administration of PTH2, increases of $\mathrm{ACTH}$ and luteinizing hormone (나) were observed in plasma [235]. In one experiment, the ventricular injection of PTH2 blunted the release of growth hormone (GH) for several hours [236]. A potential impact on both oxytocin and arginine-vasopressin, two neuropeptides heavily implicated in social interaction was observed as well [237, 238].

In particular in view of the last two peptides, it is worth mentioning that in several studies 
pth2 appears to play a role in social contexts. An increased c-fos immunoreactivity in pth2 ${ }^{+}$cells was observed in the PIL of mother rats after interacting with their pups [238]. In fact, suckling of the pups was shown to activate these cells strongly [239] and abolished pth2r activity in a $\mathrm{KO}$ animal led to a marked decrease in pup weight during development, indicative of an impairment in maternal care [240]. 


\section{Chapter 2}

\section{Materials and Methods}

\subsection{Materials}

\subsubsection{Chemicals \& biological material}

\begin{tabular}{|l|l|l|}
\hline Component & Company & Lot or $\underline{\text { Catalog number }}$ \\
\hline $\begin{array}{l}\text { 4-(4-diethylaminostyryl)- } \\
\text { N-methylpyridinium } \\
\text { iodide (4-Di-2-ASP) }\end{array}$ & Sigma-Aldrich & C D-3418 \\
\hline 4-iodophenol & Sigma-Aldrich & C I10201-25G \\
\hline Borate & Alfa Aesar & C J60803 \\
\hline Chloroform & Sigma-Aldrich & L SHBC7853V \\
\hline CaCl ${ }_{2}$ & Sigma-Aldrich & C C1016 \\
\hline Copper(II) sulfate & Sigma-Aldrich & C C1297 \\
\hline Dextran Sulfate & Sigma-Aldrich & C D8906 \\
\hline Formamide & Ambion & C AM9342 \\
\hline $\mathrm{H}_{2} \mathrm{O}_{2}$ & Sigma-Aldrich & C H1009 \\
\hline
\end{tabular}




\begin{tabular}{|c|c|c|}
\hline Heparin & Sigma-Aldrich & C H3393 \\
\hline $\mathrm{NaCl}$ & Sigma-Aldrich & C 31434 \\
\hline NaCitrate & Tocris & C 3161 \\
\hline Neomycin & Sigma-Aldrich & C N1876 \\
\hline $\mathrm{NaOH}$ & Carl Roth & C 6771.1 \\
\hline Maleic acid & Sigma-Aldrich & C M0375 \\
\hline $\mathrm{MgSO}_{4}$ & Sigma-Aldrich & C M7506 \\
\hline $\begin{array}{l}\text { One Shot TOP10 chemi- } \\
\text { cally competent cells }\end{array}$ & Thermofisher Scientific & C 404006 \\
\hline $\begin{array}{l}\text { Phusion High-Fidelity } \\
\text { DNA Polymerase }\end{array}$ & Thermofisher Scientific & L 00679781 \\
\hline $\begin{array}{l}\text { QuantiTect Reverse Tran- } \\
\text { scription Kit }\end{array}$ & QIAGEN & C 205311 \\
\hline $\operatorname{SSC}(20 x)$ & Sigma-Aldrich & C S6693 \\
\hline $\begin{array}{l}\text { SYBR Green PCR Mas- } \\
\text { ter Mix }\end{array}$ & Thermofisher Scientific & C 4309155 \\
\hline Tryptone & in-house manufactured & NA \\
\hline Taq DNA Polymerase & NEB & C M0273S \\
\hline Torula RNA & Sigma-Aldrich & C R6625 \\
\hline Triton-X 100 & BioXtra & C T9284-100ML \\
\hline Trizol & Ambion & L16655301 \\
\hline Tween20 & Sigma & C P1379-500ML \\
\hline Tyramides & Thermo Fisher & C B40953 \\
\hline yeast extract & Merck & C 103753 \\
\hline
\end{tabular}




\subsubsection{Antibodies}

\begin{tabular}{|l|l|l|}
\hline Antibody (species) & Company & Lot or Catalog number \\
\hline \hline$\alpha$-pth2 (guinea pig) & Custom antibody (PSL) & NA \\
\hline$\alpha$-znp1 (rabbit) & Synaptic Systems & C 106002 \\
\hline
\end{tabular}

\subsubsection{Devices}

\begin{tabular}{|l|l|l|}
\hline Component & Company & Lot or Catalog number \\
\hline \hline Arduino Mega 2560 Rev3 & Arduino & C A000067 \\
\hline $\begin{array}{l}\text { Frequency Generator } \\
\text { AFG3102 }\end{array}$ & Tektronix & NA \\
\hline Incubator (SHEL LAB) & Sheldon Manufacturing & NA \\
\hline $\begin{array}{l}\text { MicroAmp Fast Optical } \\
\text { 96-Well Reaction Plate } \\
\text { with Barcode }\end{array}$ & Applied Biosytsems & C 4346906 \\
\hline $\begin{array}{l}\text { MicroAmp Optical Adhe- } \\
\text { sive Film }\end{array}$ & Applied Biosystems & C 4311971 \\
\hline Microcentrifuge 5415R & Eppendorf & NA \\
\hline Real-Time PCR System & Applied Biosytems & NA \\
\hline $\begin{array}{l}\text { RNase-free 1.5 mL Mi- } \\
\text { crocentrifuge Tubes }\end{array}$ & Ambion & L 23118089 \\
\hline $\begin{array}{l}\text { RNeasy MinElute } \\
\text { Cleanup Kit }\end{array}$ & QIAGEN & C 74204 \\
\hline Soft-Ject Syringe & Henke Sass Wolf & L 18D09C8 \\
\hline ZebTEC Tank System & Tecniplast & NA \\
\hline
\end{tabular}

\subsubsection{Solution recipes}

E3 medium

\begin{tabular}{|l|l|}
\hline Reagent & final concentration \\
\hline \hline $\mathrm{NaCl}$ & $5 \mathrm{mM}$ \\
\hline $\mathrm{KCl}$ & $17 \mathrm{mM}$ \\
\hline $\mathrm{CaCl}_{2}$ & $0.33 \mathrm{mM}$ \\
\hline $\mathrm{MgSO}_{5}$ & $0.33 \mathrm{mM}$ \\
\hline
\end{tabular}

Lysogeny broth (LB) 


\begin{tabular}{|l|l|}
\hline Reagent & amount \\
\hline \hline Tryptone & $10 \mathrm{~g}$ \\
\hline yeast extract & $5 \mathrm{~g}$ \\
\hline $\mathrm{NaCl}$ & $5 \mathrm{~g}$ \\
\hline $\mathrm{ddH}_{2} \mathrm{O}$ & $1000 \mathrm{~mL}$ \\
\hline
\end{tabular}

RNA probe pre-hybridization buffer

\begin{tabular}{|l|l|}
\hline Reagent & final concentration \\
\hline \hline formamide & $50 \%$ \\
\hline $20 x$ SSC & $5 \mathrm{x}$ \\
\hline torula RNA & $5 \mathrm{mg} / \mathrm{ml}$ \\
\hline heparin & $50 \mu \mathrm{g} / \mathrm{ml}$ \\
\hline Tween20 & $0.1 \%$ \\
\hline
\end{tabular}

RNA probe hybridization buffer

\begin{tabular}{|l|l|}
\hline Reagent & final concentration \\
\hline \hline formamide & $50 \%$ \\
\hline 20x SSC & $5 \mathrm{x}$ \\
\hline torula RNA & $5 \mathrm{mg} / \mathrm{ml}$ \\
\hline heparin & $50 \mu \mathrm{g} / \mathrm{ml}$ \\
\hline Tween20 & $0.1 \%$ \\
\hline Dextran sulfate & $5 \%$ \\
\hline
\end{tabular}

20x SSC

\begin{tabular}{|l|l|}
\hline Reagent & Amount \\
\hline \hline $\mathrm{NaCl}$ & $175.3 \mathrm{~g}$ \\
\hline $\mathrm{NaCitrate}$ & $88.2 \mathrm{~g}$ \\
\hline$d d \mathrm{H}_{2} \mathrm{O}$ & $1000 \mathrm{ml}$ \\
\hline
\end{tabular}

Formamide Buffer

\begin{tabular}{|l|l|}
\hline Reagent & Amount \\
\hline \hline formamide & $500 \mathrm{~mL}$ \\
\hline $20 \times$ SSC & $100 \mathrm{~mL}$ \\
\hline$d d H_{2} O$ & $400 \mathrm{ml}$ \\
\hline Tween20 & $1 \mathrm{~mL}$ \\
\hline
\end{tabular}


Maleic Acid Buffer

\begin{tabular}{|l|l|}
\hline Reagent & Concentration \\
\hline \hline maleic acid & $100 \mathrm{mM}$ \\
\hline $\mathrm{NaCl}$ & $150 \mathrm{mM}$ \\
\hline $\mathrm{NaOH}$ & adjust to $\mathrm{pH} 7.5$ \\
\hline
\end{tabular}

Tyramide Signal Amplification buffer

\begin{tabular}{|l|l|}
\hline Reagent & Concentration \\
\hline \hline borate with $0.1 \%$ Triton-X 100, pH 8.5 & $100 \mathrm{mM}$ \\
\hline $\mathrm{H}_{2} \mathrm{O}_{2}$ & $0.003 \%$ \\
\hline dextran sulfate & $2 \%$ \\
\hline 4-iodophenol & $350-450 \mu \mathrm{g} / \mathrm{ml}$ \\
\hline tyramide & $1: 100$ \\
\hline
\end{tabular}

\subsubsection{Animal lines}

The following animal lines were used in this study:

- Konstanz wildtype strain (KN)

- Nacre phenotypic mutant strain $\left(\mathrm{mitfa}^{-/-}\right)$

- pth2 $^{\text {sa23129 }}$, pre-mature stop codon

- $p t h 2 r^{s a 11169}$, premature stop codon

- Medaka wildtype strain

- Artemia 


\subsection{Methods}

\subsubsection{Animal husbandry}

Adult and juvenile zebrafish of the lines Konstanz wildtype $(\mathrm{KN})$ and Nacre $\left(\mathrm{mitfa}^{-/-}\right)$ were housed at $28{ }^{\circ} \mathrm{C}$, using a light cycle of 14-hour light/10-hour dark and kept in 1.1 or 3.5 L ZebTEC tanks at a density of 5-35 fish of both sexes. Isolated fish and their socially-reared siblings were kept in $1.1 \mathrm{~L}$ tanks as of $6 \mathrm{dpf}$. Animals were fed three times a day with brine shrimp (Artemia salina) and/or GEMMA Micro. Vinegar eelworms (Turbatrix aceti) were fed to larval and juvenile fish. Larvae up to 5 dpf were kept in dishes (10 or 15 $\mathrm{cm}$ diameter) filled with $\mathrm{E} 3$ medium in a $28^{\circ} \mathrm{C}$ incubator with the same light/dark cycle. All animal procedures conformed to the institutional guidelines of the Max Planck Society and were approved by the Regierungsprsidium Darmstadt, Germany (governmental ID: V 5419 c 20/15-F126/1016 and V 54-19 c 20/15- F126/1013). Fish husbandry was performed by Anett-Yvonn Loos, Annette Hüttling, and Dmitrij Burgard.

\subsubsection{RNA extraction}

For RNA isolation, whole heads were used from larvae and juveniles, while brains were extracted from adults. For larvae and juveniles, 10 to 15 animals were combined per replicate, and 2 brains were pooled for adult fish. Samples were collected on dry ice in RNase-free tubes and stored in $700 \mu L$ of TRIzol (ambion) either for immediate processing or storage for up to 3 days at $4{ }^{\circ} \mathrm{C}$. Samples were homogenized using a $0.6 \mathrm{~mm}$ diameter needle attached to a $1 \mathrm{~mL}$ syringe. $140 \mu L$ of chloroform (Sigma-Aldrich) was added, and the tubes were vortexed for 15 seconds and centrifuged at $12,000 \mathrm{rcf}$ for 15 minutes at $4{ }^{\circ} \mathrm{C}$. After phase-separation, $200 \mu L$ of the clear aqueous phase was purified with the RNeasy MinElute Cleanup Kit (QIAGEN) in accordance with the manufacturers' instructions. RNA 
was eluted in water and the concentration was spectrophotometrically measured. RNA extraction for the experiments described in sections 3.3-3.5.1 was performed by Lukas Anneser, Ivan Alcantara, and Anja Gemmer. For some experiments, homogenization was performed by Jan Glüsing.

\subsubsection{Spectrophotometric and fluorometric analysis of RNA}

A spectrophotometer (NanoDrop $\left.{ }^{\mathrm{TM}} 2000\right)$ was used to determine purity and concentration of RNA and DNA samples. $1 \mu \mathrm{l}$ of sample was placed on the pedestal of the spectrophotometer and its absorbance was measured from $220-350 \mathrm{~nm}$. Aromatic bases in the structure of both RNA and DNA are characterized by peak absorbance at $260 \mathrm{~nm}$. This property can be used to derive concentrations according to the Beer-Lambert law:

$$
A=\epsilon c l,
$$

with $\mathrm{A}$ being the absorbance, $\epsilon$ a specific extinction coefficient, $\mathrm{c}$ the concentration, and I the path length.

Before the construction of libraries for NGS, sample concentration was determined by performing fluorometric analysis of RNA using the Qubit 4 fluorometer. As absorbance at $260 \mathrm{~nm}$ is not uniquely characteristic of RNA and DNA, a more targeted approach is the use of fluorescent dyes that specifically bind to nucleotide strands. $190 \mu l$ of Qubit working solution was mixed with $10 \mu l$ of supplied standards and used to calibrate the measurement. $1 \mu \mathrm{l}$ of sample was mixed with $199 \mu \mathrm{l}$ of Qubit working solution, mixed and incubated at room temperature for 2 minutes. Sample concentration was then determined by the machine by interpolating from the standards. 


\subsubsection{RNA integrity measurement}

For NGS experiments, RNA quality was assessed using the Agilent 2100 Bioanalyzer system and only samples with a RIN [241] of at least 9 were included. Before measurement, the electrodes were cleaned using $350 \mu \mathrm{l}$ of RNaseZAP and $350 \mu \mathrm{l}$ of RNase-free water for 1 minute each. Reagents were equilibrated to room temperature for 30 minutes. RNA 6000 Nano dye was vortexed and centrifuged. $1 \mu l$ was added to a $65 \mu l$ aliquot of filtered gel. The mixture was vortexed and incubated at room temperature for 30 minutes. $9 \mu \mathrm{l}$ of the gel-dye mix was loaded into a chip and homogeneously distributed using the supplied chip priming station according to manufacturer's instructions. 


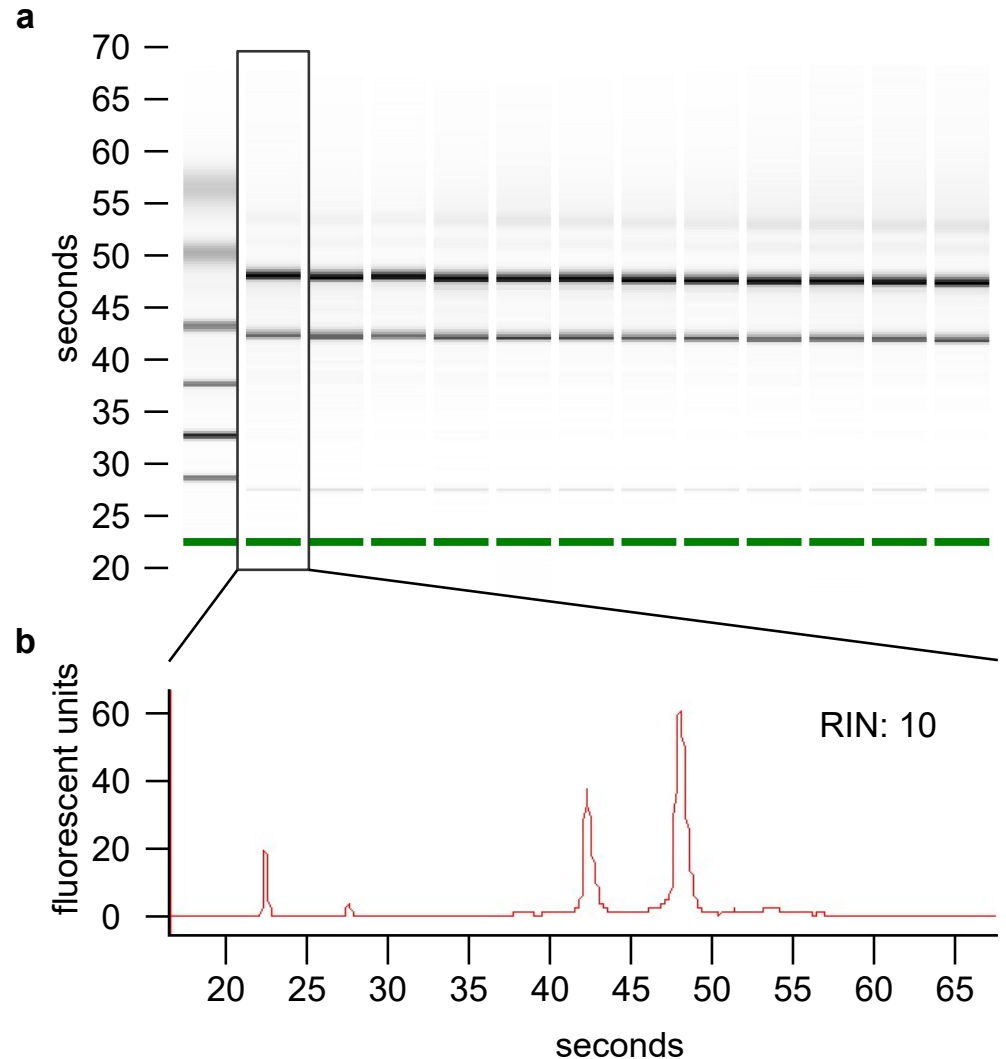

Figure 2.1: Panel a shows a typical result of the RIN measurement using the Agilent 2100 Bioanalyzer platform. The two prominent bands in each sample correspond to $18 \mathrm{~S}$ and $28 \mathrm{~S}$ ribosomal RNA. In panel $\mathbf{b}$, the intensity of these bands is shown. Area under the curve is measured for both ribosomal RNA peaks and the ratio is used to determine potential degradation of RNA. In this particular example, no degradation was observed, corresponding to a RIN of 10 .

$5 \mu l$ of RNA 6000 Nano marker was loaded into each well. Ladder and samples were heat denatured at $70{ }^{\circ} \mathrm{C}$ for 2 minutes before loading $1 \mu l$ of each into a separate well. The chip was mixed using the IKA vortex mixer for 60 seconds at $2400 \mathrm{rpm}$. Within 5 minutes, the chip was loaded into the Agilent 2100 Bioanalyzer and the run was started.

\subsubsection{Reverse transcription}

To obtain copy DNA (CDNA), 200 ng of RNA were reversely transcribed. Template RNA was mixed with gDNA wipeout buffer and RNase-free water in a total reaction volume of 14 
$\mu l$ and incubated at $42 \mathrm{C}$ for 2 minutes to remove genomic DNA (gDNA). Afterwards, the reaction was placed on ice for 2 minutes. $4 \mu l$ of $5 x$ reaction buffer, and $1 \mu l$ each of primer mix and reverse transcriptase was added to a final volume of $20 \mu l$. The reaction was incubated at $42{ }^{\circ} \mathrm{C}$ for 15 minutes and inactivated at $95 \mathrm{C}$ for 3 minutes. Afterwards, the obtained cDNA was either used directly or frozen at -20 C. The cDNA for the experiments described in sections 3.3 -3.5.1 was produced by Lukas Anneser, Ivan Alcantara, and Anja Gemmer.

\subsubsection{Next generation sequencing}

$200 \mathrm{ng}$ of RNA was used for sample preparation with the TruSeq Stranded mRNA Kit (Illumina). Libraries were prepared according to the manufacturers recommendations. In short, by using poly-T oligos attached to magnetic beads, messenger RNA (mRNA) was enriched by selecting for molecules containing poly-A parts. The mRNA was fragmented and primed with random hexamers for the conversion to CDNA. Using AMPure XB beads, double-stranded cDNA was enriched and purified. A single A nucleotide was added to the 3 -end to subsequently add i7 sequencing adapters. Incorporation of these specific sequences allowed for the identification of particular samples after sequencing. Libraries were purified, quantified using the Qubit 4 fluorometer (Invitrogen), and the average library size assessed with the Agilent HS DNA assay (Agilent). Equal amounts of all libraries were pooled and diluted to $4 \mathrm{nM}$ in $10 \mathrm{mM}$ Tris- $\mathrm{HCl}$, ph 8.5, 0.1\% Tween20. Equal amounts (5 $\mu L$ ) of library and $2 \mathrm{M} \mathrm{NaOH}$ were mixed and incubated for 5 minutes at room temperature to denature the DNA molecules. The mixture was neutralized by adding $5 \mu L$ of 200 $\mathrm{mM}$ Tris- $\mathrm{HCl}, \mathrm{pH}$ 7.0. Denatured libraries were then diluted to $1.3 \mathrm{pM}$ and loaded on a Mid Output Flow Cell (Illumina). Sequencing was performed on a NextSeq 500 machine (Illumina) using 151 sequencing cycles. 


\subsubsection{Differential expression analysis}

The obtained sequencing reads were mapped to the current Danio rerio genome assembly (GRCz11). This was done by utilizing the STAR algorithm [242]. Non-canonical intron motifs were excluded from mapping. Additionally, the options -outFilterScoreMinOverLread and -outFilterMatchNminOverLread were set to 0.25 , respectively. The reads that were mapped to individual genes were then counted with the featureCounts algorithm, using default options [243]. Gene annotation was complemented with the ENSEMBL gene ID, external gene name, description, GO-term, and entrezgene ID using the BioMart pipeline [244]. To identify differentially expressed genes, the edgeR Bioconductor package was used [32]. Since our experiments were designed in a paired manner (fish raised either isolated or under social conditions were siblings), our design matrix formed an additive model using batch identity and treatment, but no interaction term, which is typical for paired designs like this. After gene dispersion estimation, a linear model was fitted, and the effect of social isolation on gene expression was tested. The $p$-values for individual genes were multiple-testing corrected with the false-discovery rate correction method by Benjamini and Hochberg [245].

\subsubsection{Analysis of single-cell sequencing data sets}

The dataset from Raj et al. ([246]) was obtained from the Gene Expression Omnibus accession number GSE105010 and analysed in R using the Seurat package [247]. The dataset from mouse cortex and hippocampus [248] and from human cortex [249] were analysed using the USCS Cell Browser online tool [250]. In all cases, gene expression of pth2r was extracted for all cells across all cell clusters. For the zebrafish data, a bootstrap analysis was performed by randomly assigning cells to clusters and comparing the relative prevalence of pth2r in these random clusters to the actual ones. Bootstrapped 
clusters contained as many cells as the actually discovered, corresponding clusters. 1,000 bootstrap-replicates were performed. In mouse and human data, pth2r expression was binarized as either expressed or not, quantitative differences were not displayed.

\subsection{9 qPCR}

200 ng of RNA were reversely transcribed using the QuantiTect Reverse Transcription Kit (QIAGEN), adhering to the manufacturers recommendations (see section 2.2.5). cDNA was diluted 1:10 to be used as template in the qPCR reaction. Each sample was composed of $5 \mu L$ of cDNA template, $1.3 \mu L$ primers, and $6.25 \mu L$ SYBR Green PCR master mix (Applied Biosystems). For the qPCR, an initial denaturation step at $95{ }^{\circ} \mathrm{C}$ for 10 minutes, followed by 40 cycles of denaturation $\left(95^{\circ} \mathrm{C}, 15\right.$ seconds) and amplification $\left(60{ }^{\circ} \mathrm{C}, 60\right.$ seconds) on a Real Time PCR System (Applied Biosystems) was used. For all approaches, the fluorescence threshold $\mathrm{C}_{t}$ was set to 0.9 for all genes. In paired experiments, relative expression was computed by calculating the fold-change between control and experimental condition within each biological replicate. For unpaired experiments, relative expression was calculated in comparison with the mean value of the control condition. In all cases, the $\Delta \mathrm{C}_{t}$ method was used according to the following formula:

$$
\begin{gathered}
\Delta C_{t}=C_{t}(\text { gene })-C_{t}(\text { reference }) \\
\Delta \Delta C_{t}=\Delta C_{t}(\text { experimental condition })-\Delta C_{t}(\text { baseline }) \\
\text { relative fold change }=2^{-\Delta \Delta C_{t}}
\end{gathered}
$$

The following primers were used to determine expression levels: 


\begin{tabular}{|l|l|}
\hline Gene & Sequence $\mathbf{5} \rightarrow \mathbf{3}$ \\
\hline \hline pth2 forward & CCACGCAACACACAGTCAAG \\
\hline pth2 reverse & GCAAGTTACTTTGCAGAGGTC \\
\hline pth1a forward & CTCTGAGAAGCAAACGGGCA \\
\hline pth1a reverse & GCTTCCCCTGGATACAGCTC \\
\hline pth1b forward & ATGCACCAGCTCCGAAACAT \\
\hline pth1b reverse & CCTCTTGCTAATTGGCAGTCCT \\
\hline pth4 forward & ATGCACCAGCTCCGAAACAT \\
\hline pth4 reverse & CCTCTTGCTAATTGGCAGTCCT \\
\hline pthlha forward & CTGACGACGATCGTGAGGAC \\
\hline pthla reverse & GCAAGGATCCAAATCTGTGGC \\
\hline pthlhb forward & GCAGACAACGGCGTTCAGTC \\
\hline pthlhb reverse & GTTTGGACACTCCCTTCGCT \\
\hline tbp forward & GTACTCACAGGTGCCAAGGT \\
\hline tbp reverse & GATTGCGTAGGTCACCCCAG \\
\hline StAR forward [251] & TCAAATTGTGTGCTGGCATT \\
\hline StAR reverse [251] & CCAAGTGCTAGCTCCAGGTC \\
\hline$n r 3 c 1$ forward [251] & ACAGCTTCTTCCAGCCTCAG \\
\hline$n r 3 c 1$ reverse [251] & CCGGTGTTCTCCTGTTTGAT \\
\hline
\end{tabular}

Quantitative PCR in the experiments described in sections 3.3-3.5.1 was performed by Lukas Anneser and Ivan Alcantara.

\subsubsection{Social isolation}

To obtain eggs, single pairs of sexually mature zebrafish were placed in a spawning tank overnight. Embryos obtained from these setups were screened so that deformed and dead embryos animals could be removed. For isolation procedures, larvae were individually placed in a 12-well dish filled with $3 \mathrm{~mL} E 3$ at $2 \mathrm{dpf}$. To exclude the possibility of visual contact between the fish, white paper strips were placed between adjacent wells. In experiments with animals at later developmental stages, animals were placed in a 1.1 $\mathrm{L}$ tank in a ZebTEC system containing artificial plants at $6 \mathrm{dpf}$. Grey plastic insert prevented animals to see conspecifics in adjacent tanks. Socially-reared animals were kept 
in a density-matched manner ( 1 fish per $3 \mathrm{~mL}$ medium) in $10 \mathrm{~cm}$ dishes with $45 \mathrm{~mL}$ of $\mathrm{E} 3$. Unless specified in the corresponding section, the social control group always consisted of 5 fish per dish or tank. When animals received access to conspecifics, siblings from the same batch were used as social stimulus. However, in some experiments it was necessary to use mitfa ${ }^{-/-}$fish were in order to distinguish between treatment group and social stimuli.

\subsubsection{Chemosensory access to conspecifics}

To give isolated fish chemical access to conspecifics, medium was obtained from $10 \mathrm{~cm}$ dishes in which 100 siblings in $45 \mathrm{~mL}$ E3 were placed. This medium was used to replace the water in the dish of the isolated animals. The swap took place repeatedly every hour for three hours, after which time the animals were sacrificed. To allow for a direct comparison with the actual effect of conspecifics on pth2 transcription, previously isolated siblings were given access to conspecifics in the same volume for the same period of time.

\subsubsection{Visual access to conspecifics}

Larvae raised in isolation from $2-5 \mathrm{dpf}$ were subjected to a paradigm using a dual compartment chamber with dimensions $1.5 \times 3 \times 1 \mathrm{~cm}^{3}$. Adjacent compartments were prevented from chemical exchange by a transparent plexiglass barrier. In all conditions, fish were placed in one compartment of this chamber for three hours in total. To enable the fish to visually perceive conspecifics, 6 age-matched larvae were put next to the previouslyisolated fish, separated only by the transparent barrier. In the physical access condition, 5 age-matched fish were placed with the previously isolated fish in the very same compartment. In order to identify the animals reared in isolation, pigmentation mutants $\left(\mathrm{mitfa}^{-/-}\right)$ were used in these experiments. This experiment was jointly performed by Lukas Anneser, Ivan Alcantara, and Anja Gemmer. 


\subsubsection{Lateral line ablation}

$5 \mathrm{dpf}$ old previously isolated larvae were exposed to $50 \mu M \mathrm{CuSO}_{4}$ for 10 minutes or to $100 \mu M$ neomycin for 5 minutes. These chemicals were then washed out by exchanging the medium three times. Animals thus treated were then placed in a $10 \mathrm{~cm}$ dish $(45 \mathrm{~mL}$ E3) for three hours together with 14 untreated conspecifics (mitfa ${ }^{-/-}$). This was supposed to test whether social exposure still had an effect on pth2 transcription after the ablation procedure. Individual replicates of this experiment were performed by Lukas Anneser, Ivan Alcantara, and Kristina Mirkes.

\subsubsection{Exposure to artemia and medaka}

Previously isolated animals were placed in a dish with either 14 or 1,000 artemia (brine shrimp) at $5 \mathrm{dpf}$. In another experiment, previously isolated animals were exposed to either 5 medaka or 5 zebrafish. All experiments allowed the animals to freely explore their settings for three hours. The medaka experiments were performed by Lukas Anneser, Anja Gemmer, and Ivan Alcantara in the MPI for Heart and Lung Research in Bad Nauheim with the help of Simon Howard and Nana Fukuda (medaka husbandry) from the group of Didier Stainier.

\subsubsection{DASPEI live staining}

Hair cells in the lateral line were visualized by immersing live animals at $5 \mathrm{dpf}$ in $50 \mu \mathrm{M}$ 4-Di-2-ASP (Sigma-Aldrich) for 30 minutes. Medium was exchanged three times with fresh E3 before mounting the animals in agarose (1\%). Using this live staining approach, the effectiveness of ablation using neomycin or $\mathrm{CuSO}_{4}$ was assessed under an Axio Zoom.V16 (Zeiss). 


\subsubsection{Extraction and modeling of behavioral features}

Zebrafish larvae that were reared in isolation freely explored a 10-cm dish filled with 45 mL E3 medium for 10 minutes. Their movements were recorded using an acA2040-90uc camera (Basler) with a frame rate of $20 \mathrm{fps}$ at a resolution of $1.500 \times 1.500$ pixels. Videos were segmented using a common Gaussian-Mixture background-subtraction algorithm. Features extraction was performed with the OpenCV framework, using empirically adapted parameters. Manual accuracy checks were done for all videos.

\subsubsection{Mechanical stimulation}

One piezo element (UPF-76Q-220, Ekulit) was introduced per well of a 12-well dish. Its vibration frequency was regulated using a frequency generator (AFG3102, Tektronix) after signal amplification with a custom-built amplifier. Generally, the frequency generator was set to output 60 or $70 \mathrm{~Hz}$ continuously or in bursts of different lengths $(300,500,700,800$, $5000 \mathrm{~ms}$ ) with a peak-to-peak voltage of $5.0 \mathrm{~V}$. To create more complex and irregular stimulation, the frequency generator was set to external trigger control and gated using a TTL pulse delivered by an Arduino Mega 2560 Rev3 (Arduino). The Arduino board itsel was simply relaying signals from a custom-written MATLAB script, which defined pause periods during which no stimulation was delivered by sampling from a log-logistic distribution ( $\mu=$ $0.27, \sigma=0.21)$ and activity periods during which the piezo was vibrating by sampling from a normal distribution $(\mu=0.15, \sigma=0.1)$. In total, animals were exposed to this stimulation paradigm was for three hours, unless otherwise specified in the main text. To validate the vibration pattern transferred by the piezo to animals in the dish, we obtained video recordings of the artificial mechanical stimulation paradigm described above. Using a sampling rate of $180 \mathrm{~Hz}$ and a sampling period of 60 seconds, we obtained videos with a BASLER acA1920-150uc. Intensity time series were obtained for pixels at the center of the dish 
near the piezo and at the edge of the dish, where water became clearly visible by piezo activation. We applied a fast Fourier transform to analyse the frequency of our stimulation and verified that the piezo paradigm produced water movement at the intended frequency in the entirety of the dish. The piezo setup was provided by Norman Heller from the electrical workshop and Andreas Umminger from the mechanical workshop. Experiments were conducted by Lukas Anneser, Ivan Alcantara, Anja Gemme and Kristina Mirkes.

\subsubsection{Free exploration}

$5 \mathrm{dpf} f$ fish were allowed to freely explore a small dish with a diameter of $10 \mathrm{~cm}$ filled with $45 \mathrm{~mL}$ E3 medium for 10 minutes. Their movement was recorded using acA2040-90uc camera (Basler) with a framerate of $20 \mathrm{fps}$ at a resolution of $1.500 \times 1.500$ pixels. Using a custom-written python script, videos were background-subtracted with a common Gaussian-Mixture segmentation algorithm. Animals were identified using the OpenCV simple blob detector with parameters adapted for our setting. This resulted in a highly successful tracking of the individual animals. Frames in which the fish was not successfully identified by the algorithm were manually curated. Velocity of animals was computed by calculating the euclidean distance traversed between consecutive frames. Bouts were identified using the velocity track by implementing a simple peak-detection algorithm. Anxiety of animals was measured by using boldness as a proxy score that reports the time animals spent in the center of the dish as a fraction of the total time:

$$
\text { boldness }=\frac{\text { time }_{\text {center }}}{\text { timetotal }_{\text {tot }}}
$$

\subsubsection{Social preference testing in the U-chamber}

$21 \mathrm{dpf}$ were placed in the U-chamber (described in [139]) and allowed to explore it for 15 minutes. After this habituation time, 3 conspecifics were placed in one of the randomly 
chosen adjacent chambers. Those chambers are visually accessible and only separated from the main part of the dish by transparent plexiglass. Animals were recorded with a acA2040-90uc camera (Basler) with a framerate of $10 \mathrm{fps}$ at a resolution of $1.500 \times 1.500$ pixels and their trajectories over time were computed using a custom-written algorithm. Whenever the animal was entering the area in front of one of the chambers, this was counted for computing a social preference index (SPI) (see fig. 2.2):

$$
S P I=\frac{\text { time }_{\text {social }}}{\text { time }_{\text {social }+ \text { antisocial }}}
$$

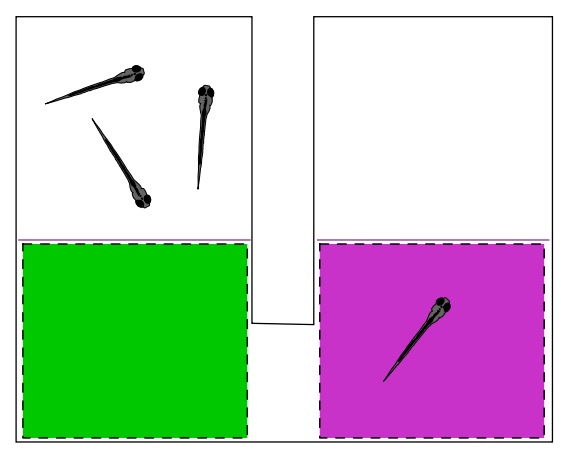

Figure 2.2: In order to compute the SPI, two areas directly in front of the adjacent compartments were identified as either social (green) or anti-social (magenta). The time spent in the green area normalized by the entire time spent in one of the two areas was used to determine social preference.

\subsubsection{Social preference testing in the open field}

At $21 \mathrm{dpf}$ of age, animals were allowed to freely explore a rectangular open field for 15 minutes. After this baseline period, 3 age- and size-matched conspecifics were placed in one of the adjacent, visually accessible chambers (separated from main chamber by plexiglass). After 15 additional minutes of exploration, the experiment ended. During the entire time, animals were recorded using the setup described above. To compute the SP] in this case, the time the animal spent in the $50 \%$ of the arena that were closer to the adjacent chamber in which conspecifics were placed, was divided by the total time spent 
in the chamber. Social preference tests were performed by Anja Gemmer, Ivan Alcantara, and Kristina Mirkes.

\subsubsection{1 gDNA extraction}

To obtain genomic DNA, tissue samples were placdd in microcentrifuge tubes with 50 $\mu l$ of $50 \mathrm{mM} \mathrm{NaOH}$. Tissue was heated to $95{ }^{\circ} \mathrm{C}$ for 20 minutes while shaking at $800 \mathrm{rpm}$. The tubes were placed on ice for 1 minute, and $5 \mu l$ of $1 \mathrm{M}$ Tris- $\mathrm{HCl}(\mathrm{pH} 8.0)$ were added for neutralization. The mixture was centrifuged for 5 minutes at 8,000 rcf to pellet the debris. Samples were either used immediately or stored at $-20{ }^{\circ} \mathrm{C}$.

\subsubsection{Polymerase chain reaction}

For PCR, HotStarTaq DNA Polymerase was used in the following setup:

\begin{tabular}{|l|l|}
\hline Component & Volume/reaction \\
\hline \hline 10x PCR Buffer & $5 \mu \mathrm{l}$ \\
\hline dNTP mix $(10 \mathrm{mM}$ of each) & $1 \mu \mathrm{l}$ \\
\hline forward primer $(10 \mu \mathrm{M})$ & $0.5 \mu \mathrm{l}$ \\
\hline reverse primer $(10 \mu \mathrm{M})$ & $0.5 \mu \mathrm{l}$ \\
\hline HotStarTaq DNA Polymerase & $0.25 \mu \mathrm{l}$ \\
\hline distilled water & $41.75 \mu \mathrm{l}$ \\
\hline template DNA & $1 \mu \mathrm{l}$ \\
\hline
\end{tabular}

The PCR of the pth2 probe sequence was conducted according to the following cycling protocol:

\begin{tabular}{|l|l|l|}
\hline Initial activation step & $95^{\circ} \mathrm{C}$ & 15 minutes \\
\hline Denaturation & $94^{\circ} \mathrm{C}$ & 30 seconds \\
Annealing & $55^{\circ} \mathrm{C}$ & 30 seconds \\
Extension & $72^{\circ} \mathrm{C}$ & 60 seconds \\
\hline Number of cycles: 35 & & \\
\hline Final extension & $72{ }^{\circ} \mathrm{C}$ & 10 minutes \\
\hline
\end{tabular}




\subsubsection{TA cloning}

For visualizing pth2, I cloned a 564 bp fragment from cDNA using the primers 5'GAAAGAGGCACCGTAGGCAA-3' and 5'-CTCTTCTGCTGGTGACCCAC-3' in a regular TA-cloning approach using HotStarTaq. The PCR product was purified using a PCR purification kit (provider) and the ligation set up in the following way:

\begin{tabular}{|l|l|}
\hline Reagent & Volume \\
\hline \hline PCR product & $2 \mu l$ \\
\hline Salt solution (supplied) & $1 \mu l$ \\
\hline Water & $1 \mu l$ \\
\hline Topo vector & $1 \mu l$ \\
\hline
\end{tabular}

The reaction was gently mixed and incubated at room temperature for 5 minutes. For transformation, OneShot cells were thawed on ice for 10 minutes. $2 \mu l$ of the ligation mix was added to the cell suspension and gently mixed by tapping the tube. After incubation on ice for 30 minutes, a heatshock at $42{ }^{\circ} \mathrm{C}$ was applied for 30 seconds and the cells were subsequently placed on ice for 2 minutes. Afterwards, $950 \mu \mathrm{l}$ of LB medium were added to the cell suspension and the mixture was incubated at 37 for 60 minutes. LB-plates infused with ampicillin were pre-warmed to $37^{\circ} \mathrm{C}$ and $100 \mu \mathrm{l}$ of the cell suspension was spreaded on the plate. The plate was then placed in an incubator at $37{ }^{\circ} \mathrm{C}$ overnight. The next day, colonies were picked for amplification in an overnight culture for subsequent plasmid preparation.

\subsubsection{Fixation of specimens}

Animals were killed by rapid immersion in ice-cold water $\left(0-4{ }^{\circ} \mathrm{C}\right)$. After cessaction of gill and heart movement, animals were transferred into $4 \%$ paraformaldehyde (PFA) in phosphate-buffered saline ( $(\overline{P B S})$. Specimens were kept in this cross-fixing chemical overnight at $4{ }^{\circ} \mathrm{C}$ while gently shaking. 
The next day, animals were washed 5 times for 5 minutes with phosphate-buffered saline with triton-X 100 (PBT) to remove PFA traces. Animals were stepwise transferred into methanol in increasing concentrations (25 - 50-75-100\% methanol in PBT) for 10 minutes each. After the last step, liquid was replaced by fresh methanol and animals were placed at $-20{ }^{\circ} \mathrm{C}$ for at least 24 hours.

For subsequent use in fluorescent in situ hybridization (FISH) or immunostainings, specimens were first permeabilized in $2 \% \mathrm{H}_{2} \mathrm{O}_{2}$ in $100 \%$ methanol and then stepwise rehydrated from $100-25 \%$ methanol in PBT in decreasing steps of $25 \%$ each for 10 minutes. Animals were then washed in PBT 2 times for 5 minutes each and subjected to downstream procedures.

\subsubsection{In-situ hybridization}

Probes were synthesized by linearizing vectors upstream of the probe sequence and in-vitro transcription with either the SP6 or T7 promoter.

After rehydration (see above), animals were permeabilized by incubating them in proteinase $\mathrm{K}$ (50 $\mathrm{g} / \mathrm{ml}$ in PBT) for 2.30 minutes at room temperature. Animals were quickly washed in PBT twice and post-fixed in 4 \% PFA for 20 minutes at room temperature while gently shaking. Traces of PFA were removed by washing 5 times in PBT for 5 minutes each. Animals were prehybridized in hybridization buffer for 60 minutes at $65^{\circ} \mathrm{C}$. Labeled RNA probes were diluted 1:500 in hybridization buffer with $5 \%$ dextran sulfate and denatured at $80{ }^{\circ} \mathrm{C}$ for 5 minutes. Hybridization buffer was then replaced by the denatured probe and probe hybridization took place for at least 15 hours at $65^{\circ} \mathrm{C}$.

The next day, probes were removed and stored at $-20{ }^{\circ} \mathrm{C}$ to be re-used. Specimens were washed 3 times 30 minutes in a mixture of $50 \%$ formamide and 2x SSC-Triton (SSC with $0.1 \%$ Triton-X 100), one time in $2 x$ SSC-Triton for 15 minutes, and 3 times 30 minutes 
in 0.2 SSC-Triton, all at $65{ }^{\circ} \mathrm{C}$. Animals were rinsed twice with PBT at room temperature and then with maleic acid buffer with $0.1 \%$ Triton-X 100 (MABT). Blocking took place for at least 2 hours at room temperature in 2x Blocking Buffer (Roche) in MABT. Depending on the labeling of the probe (digoxigenin (DIG) or fluorescein), either an $\alpha \cdot \overline{D I G}$ or an $\alpha$ fluorescein antibody conjugated with horseradish-peroxidase was diluted in the blocking solution (dilution of 1:500) and incubated with the specimens overnight at $4{ }^{\circ} \mathrm{C}$ under gentle shaking.

The antibody mix was removed and the specimens washed 6 times for 20 minutes each with PBT on a shaker. Animals were rinsed twice in $100 \mathrm{mM}$ borate $(\mathrm{pH} 8.5)$ with $0.1 \%$ Triton-X 100. A tyramide signal amplification (TSA) reaction mix was freshly prepared and tyramides were diluted 1:100. TSA reaction took place for 20 minutes at room temperature in the dark. Specimens were then rinsed 4 times thoroughly in PBT and in addition washed twice in PBT for 30 minutes each. Afterwards, specimens were either used in a downstream immunostaining for double-labeling or directly prepared for imaging.

\subsubsection{Immunostaining}

After re-hydration or FISH (see above), animals were washed once in phosphatebuffered saline with triton-X 100 and DMSO (PBTX) for 5 minutes. Blocking was performed with $10 \%$ natural goat serum (NGS) in PBTX for at least 2 hours. Primary antibodies were diluted in $1 \%$ NGS (standard dilution 1:500) and incubated overnight at $4{ }^{\circ} \mathrm{C}$.

The next day, the antibody solution was removed and the specimens washed 2 times 5 minutes each in $1 \%$ NGS in PBTX. Afterwards, the animals were washed for at least 6 hours with $1 \%$ NGS in PBTX, the solution was changed at least 6 times. Secondary antibodies were usually diluted 1:1000 in 1\% NGS in PBTX and incubated overnight at 4 ${ }^{\circ} \mathrm{C}$ while gently shaking. Specimen were then rinsed twice with $0.1 \%$ NGS in PBTX and 
then washed 3 times 30 minutes on a shaker at room temperature. To prepare samples for imaging, they were transferred to $80 \%$ glycerol in $\mathrm{PBT}$ and mounted on coverslips.

\subsubsection{Confocal imaging}

After in-situ hybridization and immunohistochemistry, animals were transferred stepwise into $80 \%$ glycerol and mounted dorsally for imaging using an inverted confocal microscope (LSM-780 or LSM-880, Zeiss, Jena, Gemany). For all conditions, animals were imaged with a 20x air objective. Lasers were used at a power range of 2 - $3 \%$. Acquisition took place in 16-bit mode as z-stacks with a $1024 \times 1024$ or $2048 \times 2048$ xy resolution covering the entire brain of a larva. Bidirectional scanning was used. Detector gain in each channel was adjusted to cover approximately $90 \%$ of the dynamic range and avoid saturated pixels.

\subsubsection{Registration to Z-Brain atlas}

The stacks obtained were co-stained with znp-1. Registration to the $z$-brain atlas was performed with the CMTK toolkit with standard settings [252, 253]. For visualization of the cell clusters, cell bodies were manually annotated in a representative registered stack using the Fiji Multi-point tool84 and overlaid with the Elavl3-H2BRFP [253] stack to visualize the zebrafish brain. 


\section{Chapter 3}

\section{Results}

\subsection{Transcriptional responses to social isolation revealed by Next Generation Sequencing}

To assess the impact of social isolation on gene transcription in the central nervous system, zebrafish were reared either in isolation or in groups of 5 starting from 2 dpf until 5, 8, 14 , or $21 \mathrm{dpf}$, reflecting the developmental trajectory from larval to juvenile fish. After these periods, the animals were sacrificed and at the later stages, the brain was extracted. At 5 and $8 \mathrm{dpf}$, whole heads (without the eyes) were obtained, rather than brains. From these tissues, RNA was extracted and used for Next Generation Sequencing. Across all developmental stages and conditions, more than 17,000 genes were detected, corresponding to approximately $65 \%$ of the annotated zebrafish genome (fig. 3.1).

Differential expression analysis revealed 319 genes, in total, that were differentially expressed between conditions. The difference between isolated and social fish was more pronounced at earlier stages. Of the differentially expressed genes, only four egr1, neuronal PAS domain protein 4a (npas4a), v-fos FBJ murine osteosarcoma viral oncogene homolog 
a

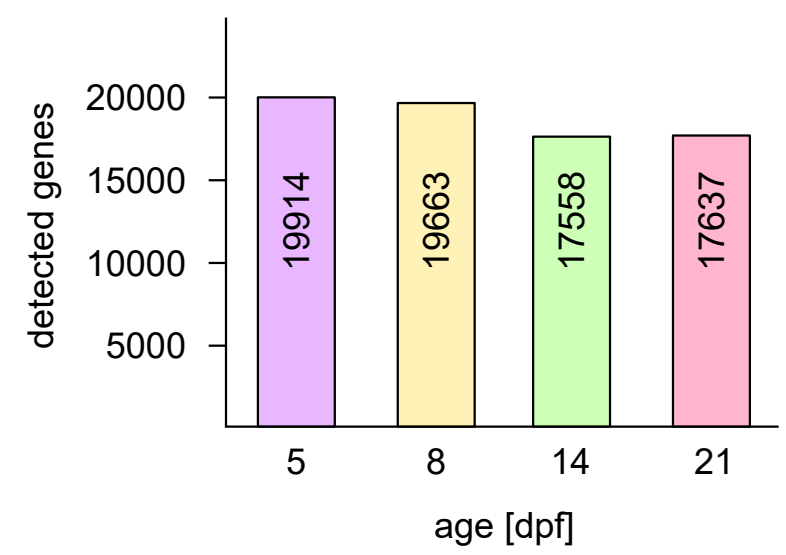

b

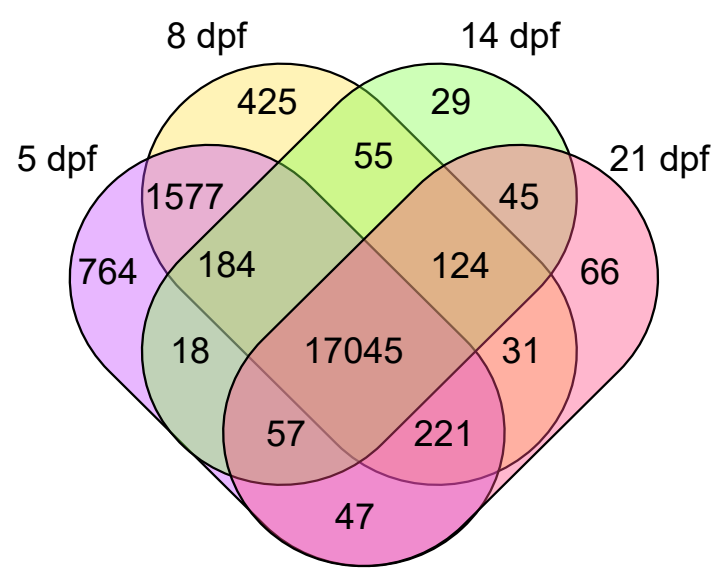

Figure 3.1: A detection criterion of at least 10 reads per gene on average for age-matched samples was used to include genes in the subsequent analysis. Panel a shows the number of genes that were detected at each age group using this criterion, panel $\mathbf{b}$ shows the overlap between developmental stages.

$A b$ (fosab), and pth2) were consistently downregulated in isolated animals across all developmental stages (fig. 3.2). Three of these (egr1, npas4a, and fosab) were IEGs, Members of this class are characterized by a rapid transcriptional response to cellular activity. In neurons, their transcription is stimulated by an increase in intracellular $\mathrm{Ca}^{2+}$ mediated by depolarization-induced opening of voltage-dependent calcium-channels. As social isolation deprives animals of a many sensory cues and the possibility to interact with conspecifics, it is to be expected that this treatment leads to a decrease in IEG transcription, so no further analysis on those genes was conducted. The differential expression of pth2 in isolated animals was independently validated by $\mathrm{QPCR}$ at several developmental stages (fig. 3.2. 

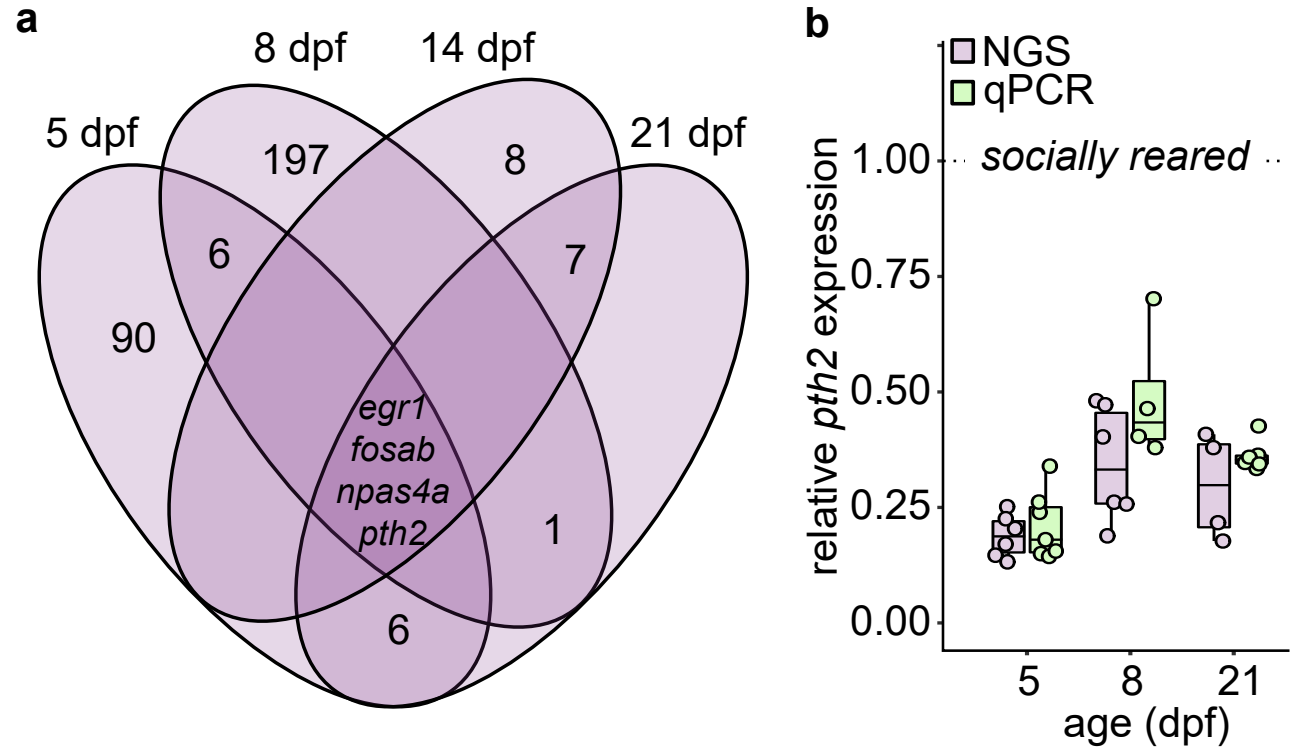

Figure 3.2: The Venn diagram in panel a shows the number of differentially expressed genes in each age group and the overlap between groups. Only four genes were consistently downregulated at all developmental stages. Panel $\mathbf{b}$ shows the validation of pth2 downregulation in isolated fish by qPCR. Adapted after [254].

\subsection{Differential expression analysis in the pth family}

The neuropeptide pth2 is a member of the pth family, comprising several genes that have been described to mediate various physiological functions, most notably the regulation of $\mathrm{Ca}^{2+}$ homeostasis. Since gene families normally emerge by duplications which might include regulatory sequences, I wanted to verify whether pth2 is the only member of its family that responds to social isolation by differential transcription. To accomplish this, I raised animals either under social conditions (groups of 15 fish) or socially isolated beginning at $2 \mathrm{dpf}$ and ending at $5 \mathrm{dpf}$. At this point, animals were sacrificed and transcription levels of the genes parathyroid hormone 1a (pth1a), parathyroid hormone $1 b$ (pth1b), parathyroid hormone-like hormone a (pthlha), parathyroid hormone-like hormone $b$ (pth/hb), and parathyroid hormone 4 (pth4) were assessed using qPCR. Only pth2 was sensitive to the social environment, while all other genes did not display differential expression following isolation (fig. 3.3). 


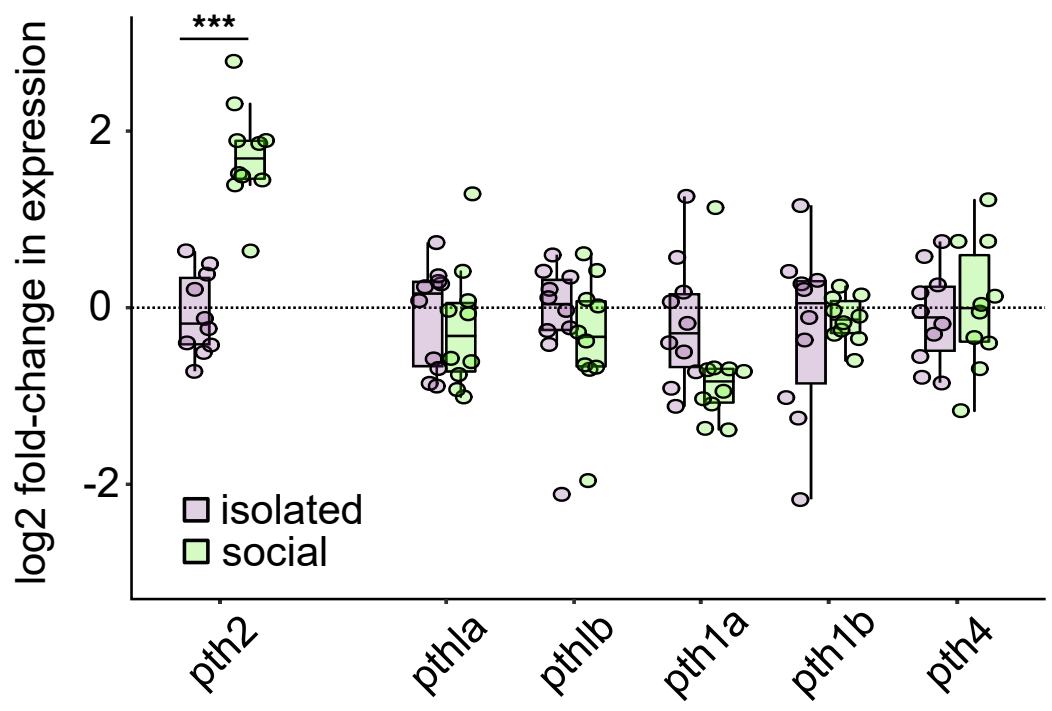

Figure 3.3: This graph shows the gene expression analysis of the pth gene family. Only pth2 was affected by social isolation ( $\mathrm{n}=10$ for all experiments, paired t-test, one-sided, Benjamini-Hochberg-corrected: pth2, $\mathrm{p}_{t=9.82}<0.001$, pthla, $\mathrm{p}_{t=-0.52}=0.62$, pthlb, $\mathrm{p}_{t=-2.55}=0.09$ pth1a, $\mathrm{p}_{t=1.55}=0.31$, pth1b, $\mathrm{p}_{t=-0.92}=0.57$, pth4, $\left.\mathrm{p}_{t=0.51}=0.62\right)$. Adapted after [254].

\subsection{Dynamics of pth2 expression}

The above results imply that gene transcription of pth2 is regulated by the presence of conspecifics. However, the deprivation of conspecifics lasted for several days, raising the question as to whether and how quickly gene expression of pth2 can adapt upon acute exposure to or deprivation of other fish. In addition, the effect was observed by only contrasting pth2 transcript levels of isolated fish with those of social animals at a particular density. However, what would happen if animals were tested at different densities - are pth2 levels more strongly regulated by higher densities of conspecifics?

\subsubsection{Time dependency of pth2 transcription}

To test the response of pth2 expression to acute changes in the social environment, I raised animals in isolation beginning at $2 \mathrm{dpf}$. At $5 \mathrm{dpf}$ (after 3 days of isolation), the fish 
were exposed to conspecifics for $30,60,180$, or 720 minutes and their pth2 levels were measured by qPCR. The above fish were compared to isolated siblings from the same batch as well as animals that were raised with conspecifics since fertilization. A statistically significant increase in pth2 levels was already observed after 30 minutes of exposure to other fish. After 720 minutes, transcript levels were indistinguishable from socially reared animals, so within 12 hours, a complete recovery was apparent.

The above results are evidence for an acute up-regulation of pth2 in response to the presence of conspecifics. Can the regulation also accur quickly in the opposite direction? For example, does the acute isolation of a socially-reared fish result in a rapid drop in pth2 levels? To test this, animals were acutely isolated after 5 days of social rearing and pth2 levels were analyzed after 3 and 6 hours. After three hours of social exposure, pth2 levels were not reduced, however, after 6 hours I observed a strong downregulation of pth2 (fig. 3.4. Transcript levels therefore were upreglated by the presence of conspecifics and their continued presence was required to maintain transcription at this increased rate. Both the upregulation of pth2 in the presence of other fish and its decrease upon acute isolation occured on a rapid timescale (within minutes to hours). 
a

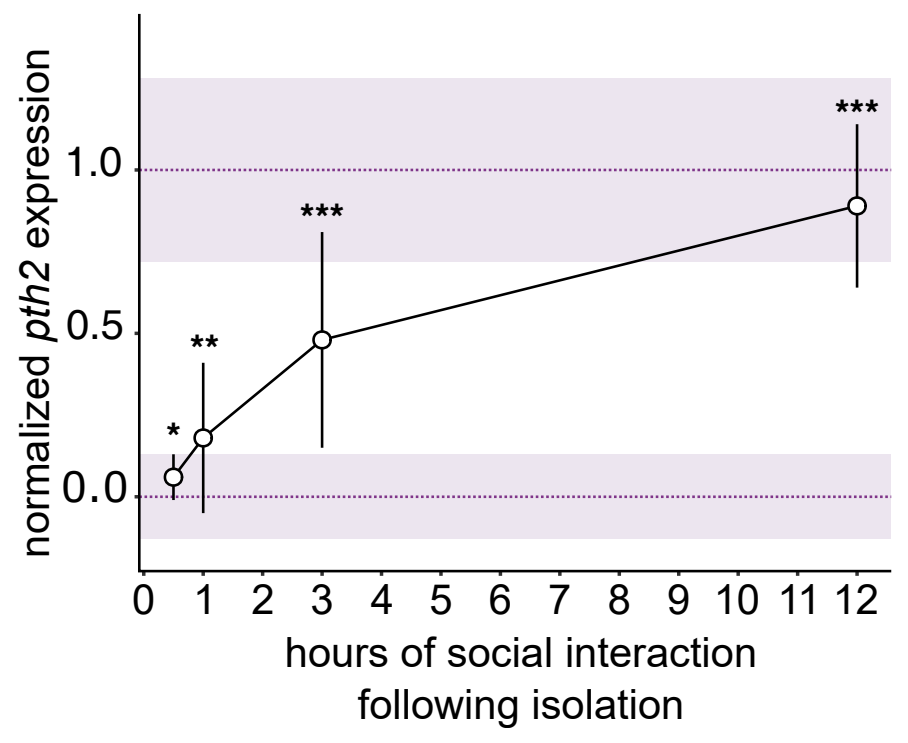

b

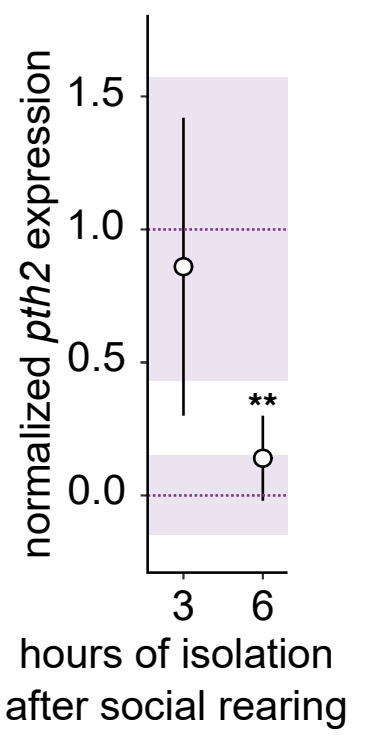

Figure 3.4: Panel a shows the upregulation of pth2 levels in previously isolated zebrafish upon exposure to conspecifics for different periods of time. Transcription levels of animals reared in isolation or with conspecifics were normalized to 0 and 1 , respectively. Values were compared to transcript levels of isolated fish (unpaired t-test, one-sided, $\mathrm{BH}$-corrected: 30 minutes, $\mathrm{p}_{(n=15, t=1.89)}<0.05,60$ minutes, $\mathrm{p}_{(n=10, t=2.45)}<0.01,3$ hours, $\mathbf{p}_{(n=10, t=4.95)}<0.001,12$ hours, $\left.\mathbf{p}_{(n=8, t=7.13)}<0.001\right)$. In $\mathbf{b}$, downregulation of pth2 in response to acute social deprivation is depicted. Animals were isolated for 3 or 6 hours, pth2-levels were normalized as in a). Values were compared to transcript levels of socially reared fish (unpaired t-test, one-sided, BH-corrected: 3 hours, $\mathbf{p}_{(n=9, t=-1.04)}=0.31,6$ hours, $\left.\mathbf{p}_{(n=3, t=-3.66)}<0.01\right)$. Adapted after [254].

\subsubsection{Transcription of pth2 is dependent on conspecific density}

The quick, bidirectional response of pth2 to the presence or absence of conspecifics was observed using a group of 15 zebrafish as the social control condition. However, does pth2 respond to other fish in a merely qualitative or also a quantitative manner? That is, given a situation in which e.g. twice as many animals are present in the same volume, how much does pth2 increase?

To test this, I raised animals either in isolation or in groups of $5,20,50$, or 100 fish per tank until $8 \mathrm{dpf}$. At this point, animals were sacrificed and their pth2 transcript levels were 
measured and compared.

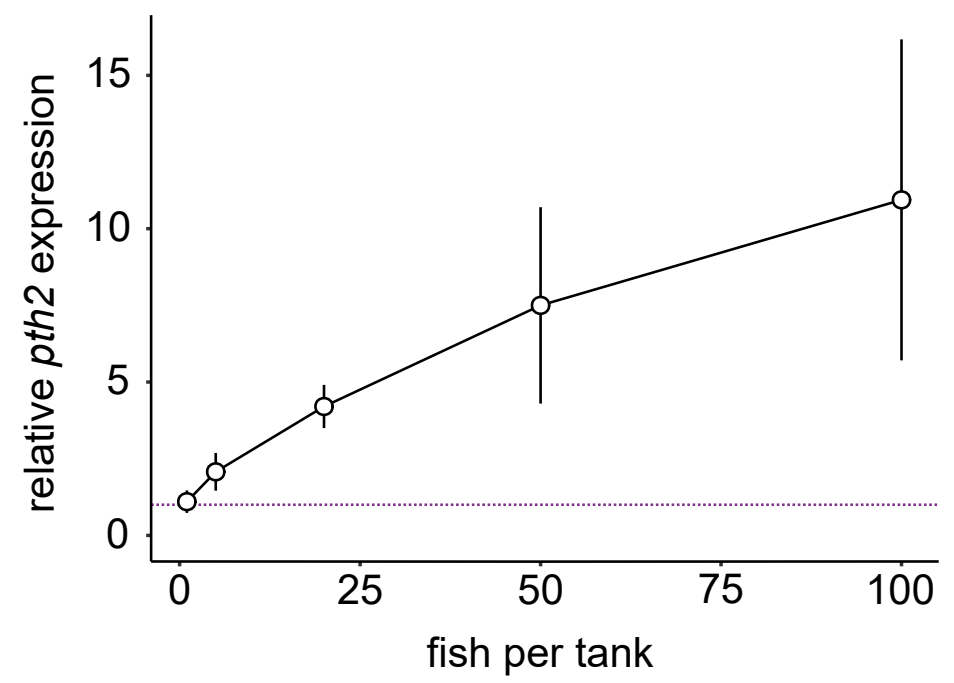

Figure 3.5: The transcription levels of pth2 were positively correlated with the number of conspecifics present. This graph shows pth2 levels after raising animals in different group sizes until the age of $8 \mathrm{dpf}$. The dashed line indicates pth2 levels of isolated animals. Adapted after [254].

The results indicated that pth2 increases with the number of conspecifics present in a sublinear manner. Saturation was not observed even in the condition of 100 animals per tank. These data imply a quantitative relationship between the number of conspecifics present and pth2 levels.

As the number of interactions between animals and the amount of sensory cues perceived is contingent upon how close animals are to each other, it is possible that a small number of animals in a small volume has the same effect than a large number of animals in a large volume - is it the absolute number of animals that is perceived or their density? To investigate this difference, 5 previously isolated animals were together acutely introduced to receptacles of different volumes $(3,15,45$, or $135 \mathrm{ml})$ for three hours. Then, pth2 levels were measured by qPCR. This way, the absolute number of fish was held constant but their density varied. I found that pth2 levels were indeed sensitive to the density of conspecifics: the larger the volume of the receptacle, the lower was the observed social environment- 
induced increase in pth2. This finding indicates that in fact the density of animals is of relevance for the regulation of pth2 transcript levels 3.6 .

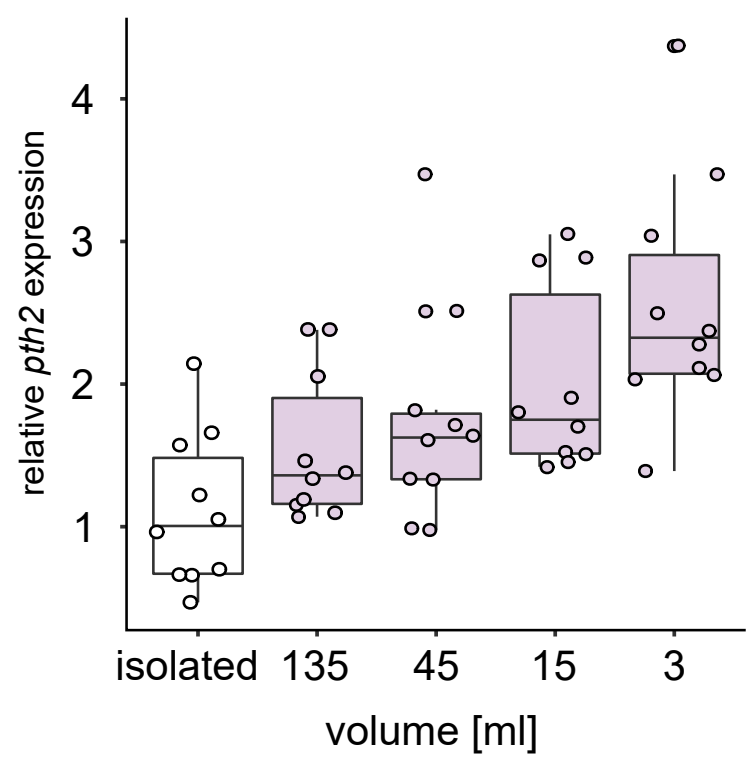

Figure 3.6: Previously isolated fish (5 dpf) were exposed to conspecifics in groups of 5 for 3 hours in different volumes. Transcript levels of pth2 were negatively correlated with volume, indicating that it is the density of conspecifics that is of relevance for regulating transcription. 10 replicates were obtained in this experiment.

\subsubsection{Modeling the expression landscape of pth2}

The results above demonstrate that the neuropeptide pth2 is quickly and bidirectionally regulated by the density of conspecifics. However, if these results are reliable, it should be possible to predict changes in pth2 levels based on knowledge of the above-specified variables:

- Time of social exposure

- Number of conspecifics in a given environment

- Volume that is available to the animals to explore. 
As the relationship between pth2 levels and these variables appears to be of a sigmoid shape (see figures 3.4 a, 3.5, 3.6, a logarithmic data transformation should result in a linear relationship, allowing for multivariate linear regression. I used the datasets I had previously acquired to fit a model of the form

$$
p t h 2=a+b * \log _{10} V+c * F+d * \frac{1}{\log _{10} T}
$$

with $\mathbb{D}=\left\{x \in \mathbb{Q}_{+} \mid x \geq 1\right\}$, as we assume isolated animals possess the lowest-possible amount of pth2. I describe all other expression levels in relation to this value. Furthermore, as a linear relationship could result in negative outcomes, a lower boundary is required, which is instantiated by our definition of the domain of the function $\mathbb{D}$.

In this formula, pth2 indicates relative transcript levels, $V$ the available volume, $F$ the number of fish in a given experiment, and $T$ the time animals are exposed to each other. The coefficients were fitted as $b=-0.62, c=0.07$, and $d=-2.02$ with an intercept of -2.53 (fig. 3.7).

a

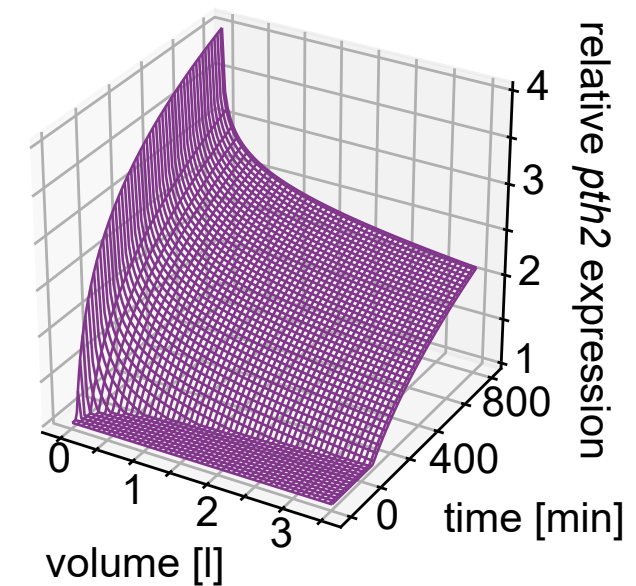

b

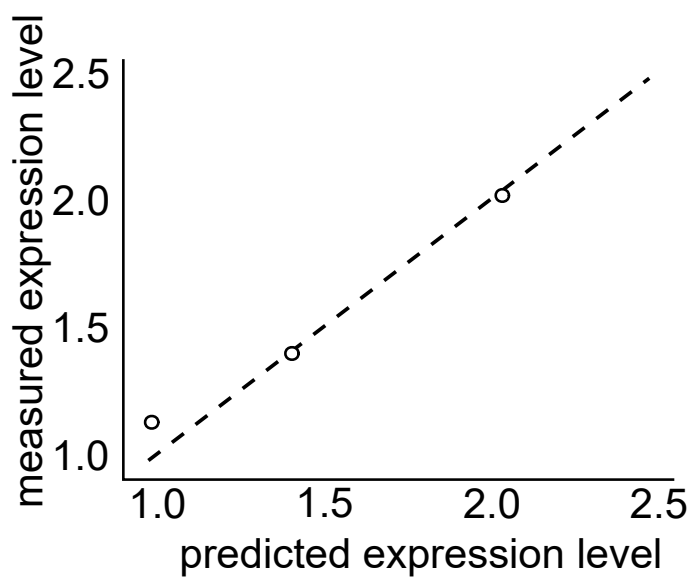

Figure 3.7: Panel a shows the expression landscape of pth2 according to a multivariate linear regression model that incorporates time of social exposure and volume in which the animals move to predict changes in pth2 levels. In this particular instance, transcript levels were modeled for a group of five fish. In panel $\mathbf{b}$, I measured pth2 expression after different paradigms and found predictions and measurements to be tightly correlated. 
Using this model, I was able to accurately predict changes in pth2 levels after exposing animals to conspecifics in specific settings (fig. 3.7 b). Taken together, these findings demonstrate that different densities lead to specific steady-state transcription levels (fig. 3.5). However, within the first hours of exposure to conspecifics, the increase of transcripts is positively correlated with density, so that pth2 levels rise faster at higher densities.

\subsubsection{Dynamic regulation of $p$ th2 occurs at all developmental stages}

So far, the dynamic nature of pth2 transcription has been demonstrated at earlier developmental stages. Do these observations also hold true for juvenile and adult fish? To test this, animals were raised until 21 dpflor 3 months post fertilization (mpf), respectively, either in isolation or in groups of 10 . At $20 \mathrm{dpf}$, socially reared animals were placed in isolation for 24 hours. After this period, pth2 levels in the fish isolated for 20 days and then exposed to conspecifics for just 24 hours were indistinguishable from animals that were reared in isolation from $2-21 \mathrm{dpf}$. This indicates that pth2 is still dynamically regulated at the juvenile stage (fig. 3.8 a). At $3 \mathrm{mpf}$, previously isolated animals were placed in groups of 10 conspecifics for 24 hours and socially reared fish were isolated for the same duration. Afterwards, pth2 levels were measured. These acute treatments were sufficient to, in the case of acute social exposure, raise pth2 levels to those of animals that have been under social conditions for their entire life. In the case of acute social isolation, pth2 levels were strongly and significantly decreased (fig. 3.8 b). These data demonstrate that the dynamic regulation of pth2 can be observed throughout all developmental stages of the zebrafish lifespan. 
a

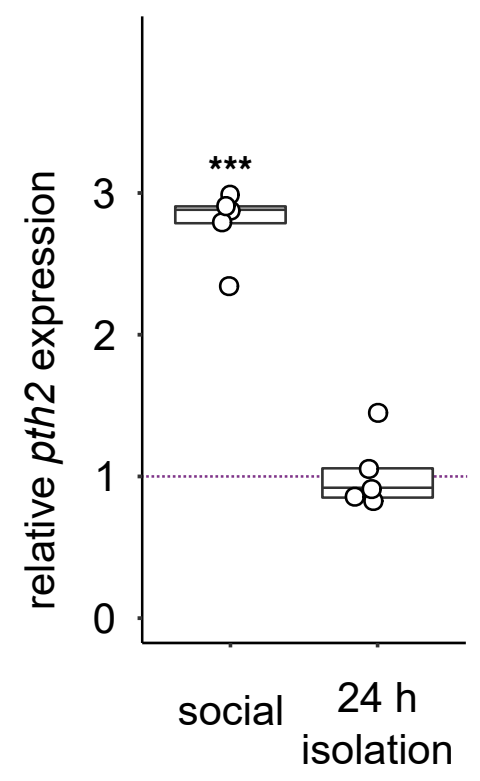

b

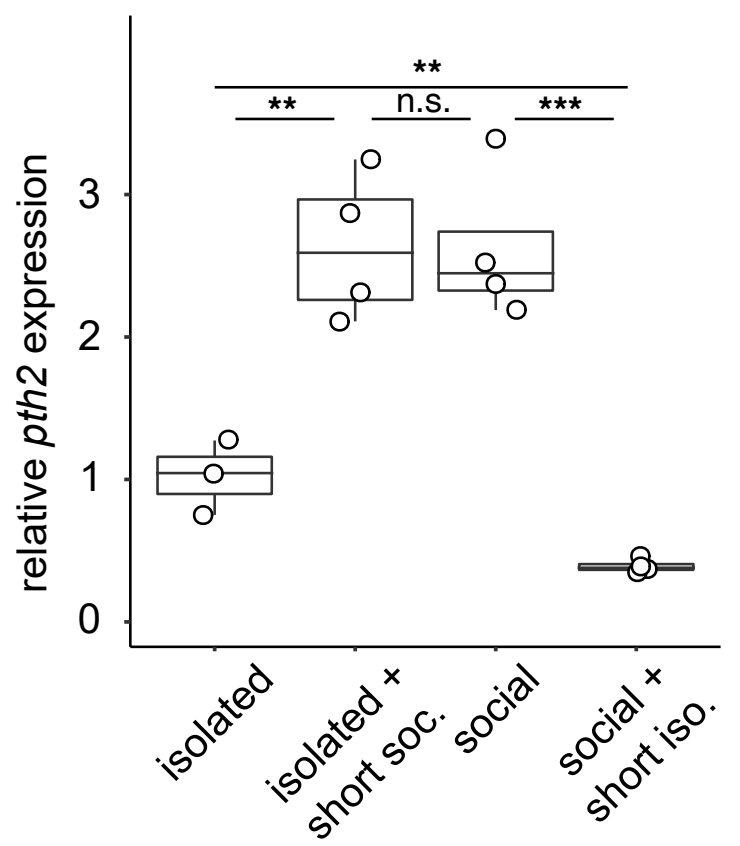

Figure 3.8: Animals were raised either in isolation or in groups of 10 to the juvenile stage (a) or adulthood (b). At $21 \mathrm{dpf}$, transcript levels after 24 hours of isolation were similar to animals that were isolated since $2 \mathrm{dpf}$, indicated by the dashed line $(n=5$ for all, paired t-test, one-sided, $\mathrm{BH}$-corrected: $24 \mathrm{~h}$ isolation, $\mathrm{p}_{t=-0.01}=0.98$, social, $\mathrm{p}_{t=-23.6}<0.001$ ). In panel $\mathbf{b}$, the impact of the social environment on adults is shown. After three months, isolated fish were exposed to conspecifics for one week (isolated + short soc.), socially reared fish were isolated for the same duration (social + short iso.). For isolated condition, $\mathrm{n}=3$, for all other $\mathrm{n}=4$ (unpaired t-test, one-sided, BH-corrected. Isolated vs isolated, short social: $\mathrm{p}_{t=-5.49}<0.01$, social vs isolated, short social: $\mathrm{p}_{t=-0.04}=0.48$, social vs social, short isolation $\mathrm{p}_{t=6.46}<0.001$ ), isolated vs social, short isolation: $\mathrm{p}_{t=6.46}<0.01$. Adapted after [254].

\subsection{Perception of conspecifics}

The presence of conspecifics is sufficient to drastically change the expression pattern of the neuropeptide pth2. How are other fish perceived by the animal? Several channels of sensory information are available:

- Visual cues

- Olfactory/chemical cues 
- Mechanical cues

To determine the mechanism, I examined the potential contribution of each specific sensory domain indicated above to the social regulation of pth2 levels.

\subsubsection{Chemical exposure to conspecifics does not regulate pth2}

The presence of fish might induce changes in the chemical composition of the medium. For example, specialized molecules or waste products might be secreted and serve as signals that can be detected by other animals, including conspecifics. The perception of these molecules could lead to differential expression of pth2. In adult zebrafish, rapid olfactory discrimination learning has been described. Based on the delivery of different odors, animals were able to learn where they would receive food [255]. Already in larval fish, chemosensation plays an important role and 5 - 6 dpf animals require olfaction to recognize kin [161].

To test the hypothesis that pth2 is regulated by the perception of conspecifics via olfaction, I raised animals in isolation from $2-5 \mathrm{dpf}$. At this point, the medium in the animals' dish was exchanged with medium obtained from a dish in which animals had been raised in high density (100 fish in $45 \mathrm{ml} \mathrm{E3,} \mathrm{kin-imbued} \mathrm{medium).} \mathrm{Every} \mathrm{hour,} \mathrm{medium} \mathrm{was} \mathrm{ex-}$ changed with this kin-imbued medium for a total duration of 3 hours. Afterwards, pth2 levels were measured using qPCR. The swap with kin-imbued medium had no measureable effect on pth2 transcript levels, which were indistinguishable from untreated, isolated fish (fig. 3.9). 
a

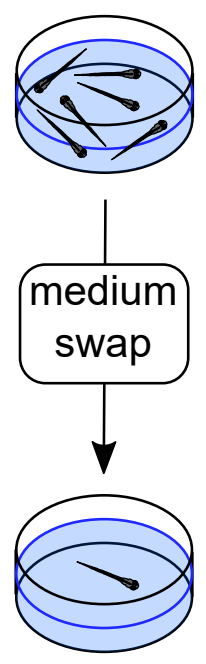

b

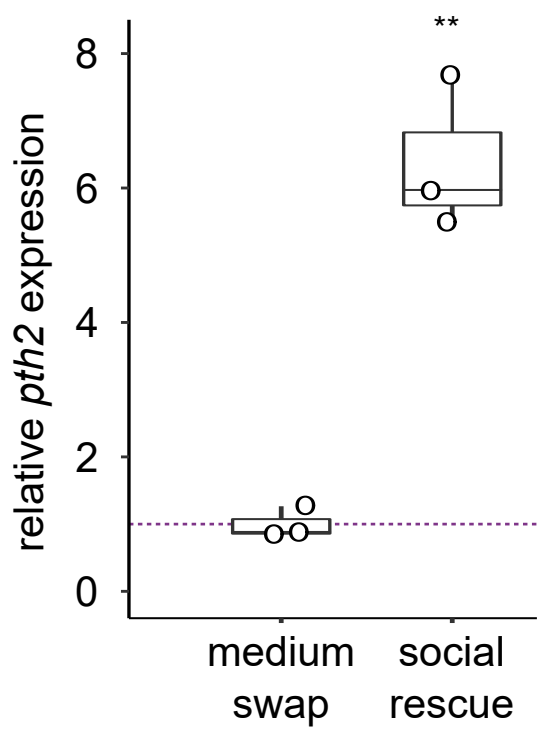

Figure 3.9: Panel a shows the schematic approach: Previously isolated animals were given chemical access to conspecifics via a medium swap. The boxplots in $\mathbf{b}$ show the impact this of chemosensory access to conspecifics has on pth2 levels. Expression is plotted in relation to isolation-reared siblings (dashed line). For all conditions, $n=3$ (paired, one-sided t-test, BH-corrected. Medium swap: $\mathrm{p}_{t=0.18}=0.87$, social rescue:

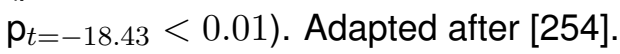

\subsubsection{Visual access to conspecifics is insufficient to trigger pth2 tran- scription}

Zebrafish have a good sense of vision and are indeed strongly dependent on visual input to navigate their surroundings and capture prey [256, 257, 258]. In addition, they develop a social preference towards conspecifics that is purely visually mediated early on in life [137, 139]. Furthermore, juvenile zebrafish appear to possess neural networks that are tuned towards recognizing movement patterns that are stereotypical of conspecifics [259]. Work from our lab has shown that visual access to conspecifics has rewarding properties for animals as early as $6 \mathrm{dpf}$ [137]. To test whether visual cues from conspecifics contribute to the regulation of pth2, animals were raised in isolation from $2-5$ or $21 \mathrm{dpf}$. They were then individually placed in a custom-built experimental setup consisting of two 
adjacent chambers that were separated by a transparent barrier that allowed for visual cues to be obtained from the adjacent chamber. Conspecifics were added either to the chamber in which the isolated animal was placed in or in the adjacent chamber, so that either direct access to conspecifics was possible or only visual access was given. Four different configurations were tested:

1. Isolation - No conspecifis were added to any chamber, so that the experimental animal was isolated throughout the entire experiment.

2. Physical access - Addition of 5 conspecifics to the chamber of the previously isolated animal, allowing for direct interaction.

3. Visual access - Addition of 6 conspecifics to the adjacent chamber, so that only visual access was allowed.

4. Visual \& physical access - Conspecifics were placed in both chambers, so that visual access to twice as many fish was provided. 
a

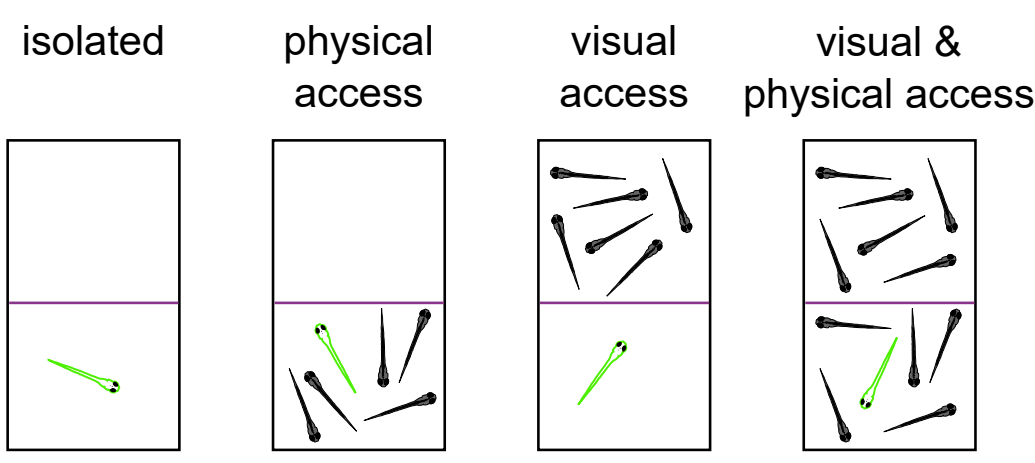

b

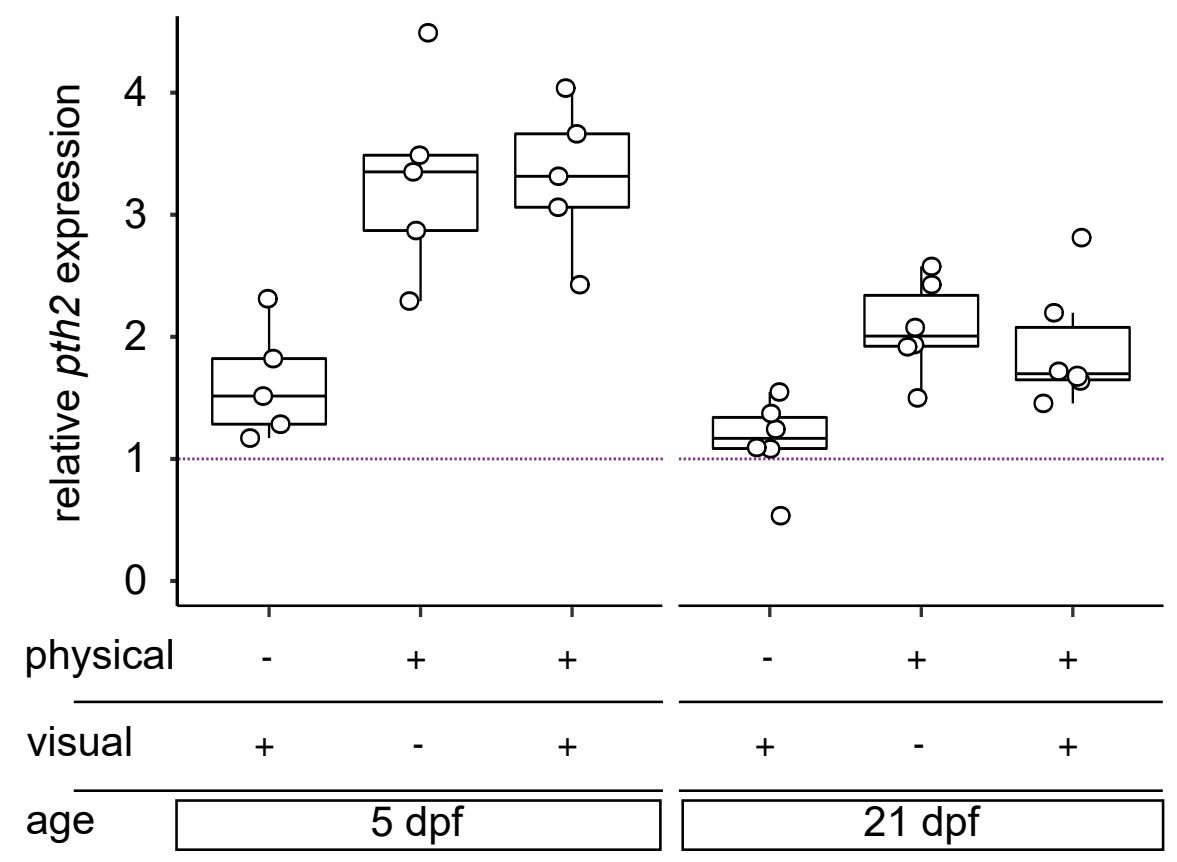

Figure 3.10: Previously isolated animals (depicted in green) were placed in four different conditions, as shown in a: They were given direct physical access to conspecifics, only visual access through plexiglass, both kinds of access or were kept in isolation. The boxplot in $\mathbf{b}$ shows the relative expression of pth2 in response to these treatments. The impact of visual and physical access as well as the interaction of both was evaluated using a 2-way ANOVA. At $5 \mathrm{dpf}, \mathrm{n}=5$ for all groups. For visual access, $\mathrm{p}_{F=3.21}=0.09$, physical access, $\mathrm{p}_{F=53.94}<0.001$, visual-physical interaction, $\mathrm{p}_{F=2.93}=0.11$. At $21 \mathrm{dpf}, \mathrm{n}=6$ for all groups. For visual access, $\mathrm{p}_{F=0.0002}=0.99$, physical access, $\mathrm{p}_{F=30.64}<0.001$, visual-physical interaction, $\mathrm{p}_{F=0.60}=0.44$. Adapted after [254].

If visual access regulates pth2 transcription, we would expect condition 2 and 3 to yield similar increases in pth2. Condition 4 should result in the strongest increase, as it offers visual access to the largest number of animals. However, there was no significant increase 
in pth2 levels when animals only had visual access to conspecifics. Paired visual \& physical access did not result in a further increase when compared to physical access alone (fig. 3.10). This indicates that vision is not the sensory modality that regulates pth2 levels following social isolation. If this was correct, then the regulation of pth2 by an animal's social environment should be observed even in total darkness, making it impossible to receive visual cues from other fish. Indeed, when I placed previously isolated fish together with conspecifics in darkness for three hours, the usual increase of pth2 was observed, providing further evidence that visual cues are not regulating the transcription of $p$ th2 (fig. 3.11.

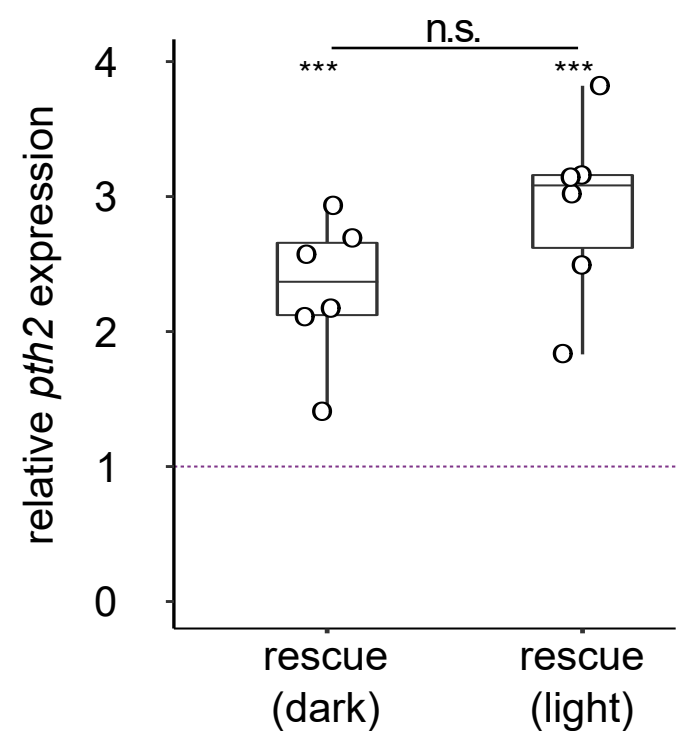

Figure 3.11: When previously isolated animals were exposed to conspecifics either in complete darkness or with ambient light, pth2 increased in both conditions. In both cases, six replicates were performed. Horizontal dashed line indicates expression level in isolated fish. For social exposure in darkness, $\mathrm{p}_{t=7.59}<0.001$, social exposure under illumination, $\mathrm{p}_{t=10.04}<0.001$, comparison between social exposure under illumination and in darkness, $\mathrm{p}_{t=-1.42}=0.22$. Adapted after [254]. 


\subsubsection{Ablation of the lateral line with neomycin and $\mathrm{CuSO}_{4}$}

Movement under water induces the propagation of waves. Percerption of water motion can thus be highly informative about the presence of prey or predators. Zebrafish can perceive pressure gradients in water via the lateral line. This organ consists of mechanosensitive neuromasts that are deposited along the head and the side of the animal [187]. Hair cells are deflected by water flow resulting changes in firing frequency, thus encoding strength of the wave. The orientation of the neuromasts can additionally encode directionality of water motion. If conspecifics are perceived via this system, then ablation of the lateral should abolish the increase in pth2 normally induced by the presence of other fish. To test this, a reliable method was required to specifically interfere with the function of the lateral line while leaving other sensory systems intact. It has been reported that aminoglycosides and heavy metals such as $\mathrm{CuSO}_{4}$ can be used to ablate neuromast cells rather specifically [260, 261, 262]. I treated 5 dpf zebrafish with low doses of neomycin (100 $\mu \mathrm{M}$, 5 minutes) or $\mathrm{CuSO}_{4}(50 \mu \mathrm{M}, 10$ minutes) and tested the incorporation of 4-Di-2-ASP, a cationic dye used for live staining of hair cells [263]. 4 Both treatments resulted in a stark

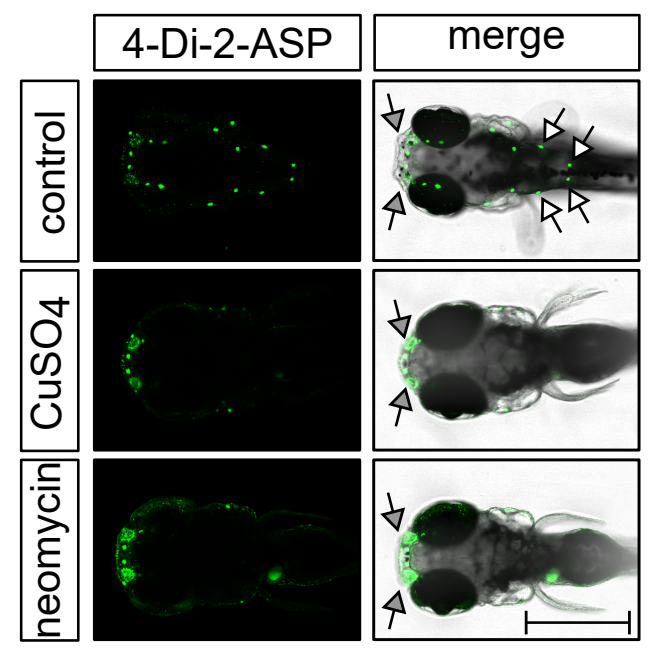

Figure 3.12: Visualization of hair cells in the lateral line (white arrowheads) and the olfactory epithelium (grey arrowheads) in $5 \mathrm{dpf}$ fish under control conditions and after treatment with neomycin or CuSO4. Scale bar $=1 \mathrm{~mm}$. Adapted after [254]. 
decrease in the fluorescent signal obtained from 4-Di-2-ASP indicating the likely loss or compromised health of the lateral line. 4-Di-2-ASP is not specific for the lateral line, it is also incorporated e.g. in the olfactory bulb [264]. This area was not affected by the treatment, suggesting a specific effect of neomycin and $\mathrm{CuSO}_{4}$

\subsubsection{Ablation of the lateral line prevents pth2 regulation by con- specifics}

Under normal conditions, exposure to conspecifics induces an increase in pth2 transcript levels in previously isolated fish. Is this still the case in fish whose lateral line has been ablated using neomycin or $\mathrm{CuSO}_{4}$ (fig. 3.12)? Animals that were reared in isolation were quickly exposed to either neomycin or $\mathrm{CuSo}_{4}$ and then - after thorough washing placed with 14 untreated conspecifics of a different phenotype ( $\mathrm{KN}$ with mitfa $^{-/-}$) for three hours. This period of social exposure is normally sufficient to induce a strong increase in pth2. However, when transcript levels were measured, no difference was found between the neomycin or $\mathrm{CuSO}_{4}$ treated previously isolated fish and their continuously isolated siblings (fig. 3.13). These data indicate that perception via the lateral line is necessary for the regulation of pth2 that is observed in the presence of conspecifics. Compromising the lateral line's function by ablation prevents the expected increase of pth2 transcript levels. Thus, it is likely that the water motion induced by the movement of fish is the salient cue driving pth2 regulation. 


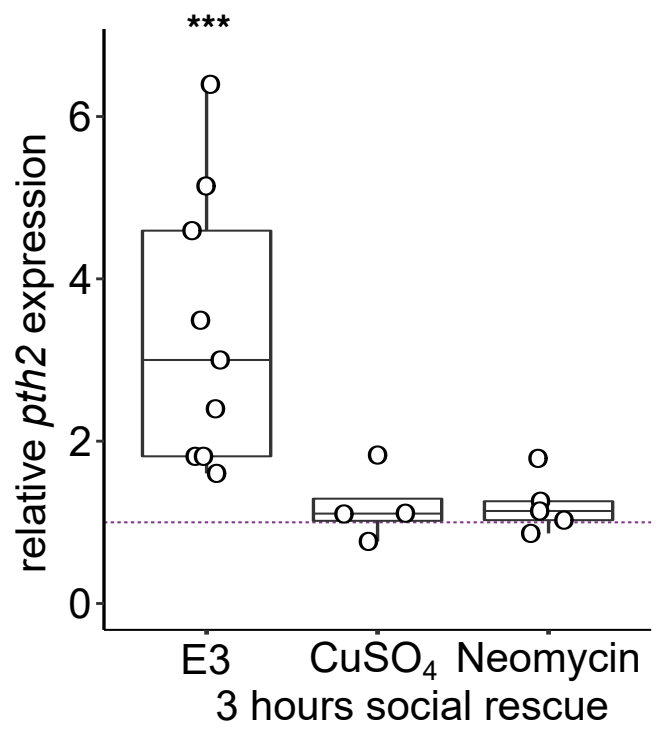

Figure 3.13: Boxplot depicts relative pth2 levels after exposure to conspecifics for three hours. Dashed line indicates pth2 levels in isolated animals. No difference to this baseline level was detected in animals whose lateral line was ablated. For control animals, $\mathrm{p}_{(n=9, t=6.57)}<0.001$, after CuSO4 treatment, $\mathrm{p}_{(n=5, t=1.39)}=0.25$, after neomycin treatment, $\mathrm{p}_{(n=5, t=1.35)}=0.25$. Adapted after [254].

\subsubsection{Response to the presence of different species}

Mechanosensation via the lateral line has been proposed to play a role in social interaction [175], but it is not clear how precisely an animal can represent its environment using this sense. Given the above findings, the lateral line appears to be necessary for the perception of conspecifics, which regulates the transcription of pth2. However, what specific features of conspecific movement are relevant for this? Is the increase in pth2 brought about by an increased turbulence in the water? To test this, previously isolated animals (5 dpf) were exposed to two different species whose locomotion patterns are either very distinct from or rather similar to zebrafish swimming patterns.

First, previously isolated animals were exposed to larvae of the brine shrimp (artemia salina). These animals move by rhythmic strokes of their appendages. Neither a small ( $\mathrm{n}$ $=14$ in $45 \mathrm{ml}$ ) or a huge large number of artemia $(\mathrm{n} \approx 1,000$ in $45 \mathrm{ml})$ was able to rescue 
pth2 levels in previously isolated fish (fig. 3.14a).

Medaka (Oryzias latipes) are small fish that are comparable in size to zebrafish. When previously isolated fish were exposed to size-matched medaka for 3 hours, an increase in pth2 levels was observed that was similar to exposure to zebrafish siblings (fig. 3.14 b c). This suggests that there is a specific set of mechanical features that are recognized by the lateral line system and downstream neural circuits, enabling the zebrafish to extract information about its environment and the presence of conspecifics, but not necessarily selective to those - other fish of similar size and with comparable locomotion features are recognized as well.

a

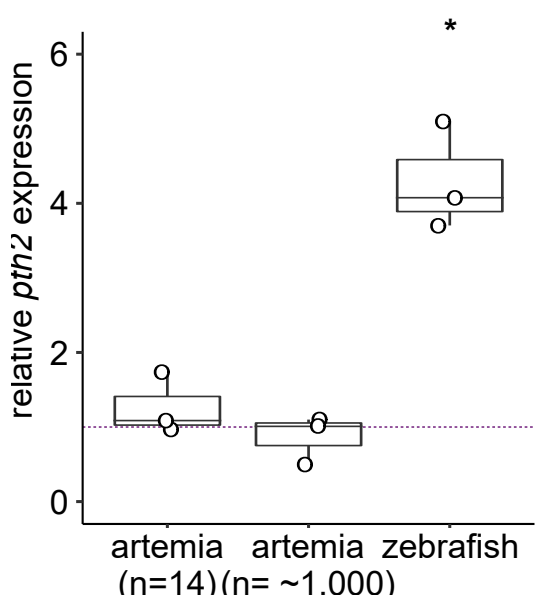

b
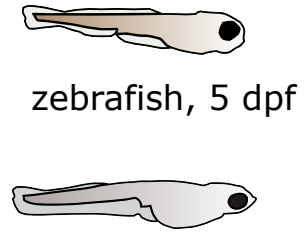

medaka, $8 \mathrm{dpf}$

$5 \mathrm{~mm}$ c

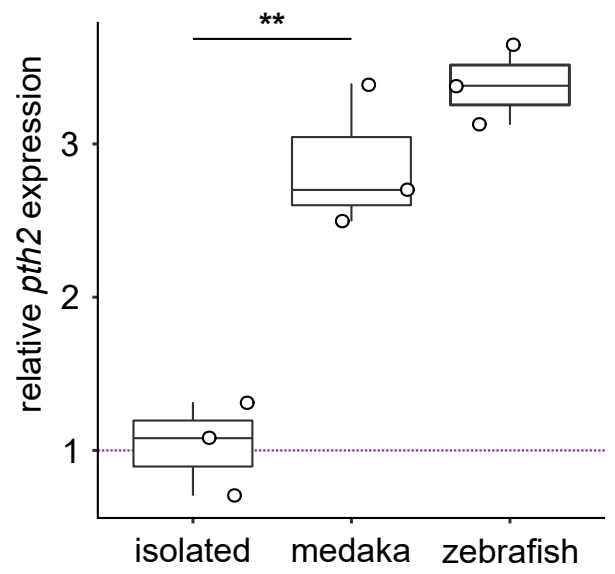

Figure 3.14: Panel a shows the impact of the presence of artemia on pth2 levels in previously isolated zebrafish. Neither a small number $(n=14)$ nor the excess of artemia $(n \approx 1,000)$ was sufficient to increase transcript abundance. Paired, one-sided t-test, $\mathrm{BH}$-corrected, $\mathrm{n}=3$ for all conditions. With 14 artemia, $\mathrm{p}_{t=1.12}=0.38$, with approximately 1,000 artemia, $\mathrm{p}_{t=-0.79}=0.51$, with 14 zebrafish, $\mathrm{p}_{t=15.29}<0.05$. In panel $\mathbf{b}$, a cartoon of zebrafish and medaka are shown at the developmental stage they were used in the experiment. Medaka grow slower than zebrafish, that's why older fish were used in this paradigm. Panel $\mathbf{c}$ shows the increase in pth2 levels in previously isolated zebrafish upon exposure to either medaka or siblings for three hours. For medaka, $\mathrm{p}_{t=5.69}<0.01$, for zebrafishp $_{t=10.2}<0.001$. Number of replicates $=3$ in all cases. Panel a adapted after [254]. 


\subsection{Movement parameters of isolated and social animals}

The results outlined above indicate that a particular kind of mechanical stimulation triggers transcription of the neuropeptide pth2. This suggests that if an artifical stimulation paradigm is used that sufficiently closely mimicks these relevant features, pth2 levels should increase in previously isolated animals, even in the absence of other fish.

\subsubsection{Artificial mechanical stimulation increases pth2 transcription}

To test whether specific locomotion features induced transcription of pth2, previously isolated animals ( $5 \mathrm{dpf})$ were placed individually in wells $(3 \mathrm{ml}$, diameter of $1.5 \mathrm{~cm})$. A piezo actuator was lowered into the well and activated via a frequency generator whose output could be controlled using custom-written MATLAB code. Using this setup, different stimulation paradigms and their impact on pth2 were tested. According to published sources, larval zebrafish accelerate by moving their tail with frequencies at about $60-70 \mathrm{~Hz}$ [265], so the initial stimulation frequency was set to this range. Continuous activity of the piezo at $70 \mathrm{~Hz}$ for three hours did not increase transcript levels significantly. The same experiment performed in kin-imbued water also failed to alter pth2 abundance. In an attempt to recreate the presence of several mechanical stimuli (which would be the case if several fish were present in the well), the piezo was adapted to consist of two elements, with or without flexible extensions. Delivery of mechanical stimulation was performed in discrete epochs with the same inter-stimulus interval $(300,500,700,800,5000 \mathrm{~ms})$. None of these paradigms brought about an increase in pth2 levels, further indicating that a distinct set of features is necessary to induce transcription (fig. 3.15 a). Would it be possible to induce

an increase in pth2 transcript levels by using a stimulation paradigm that incorporated the stochasticity of actual zebrafish locomotion? 
a

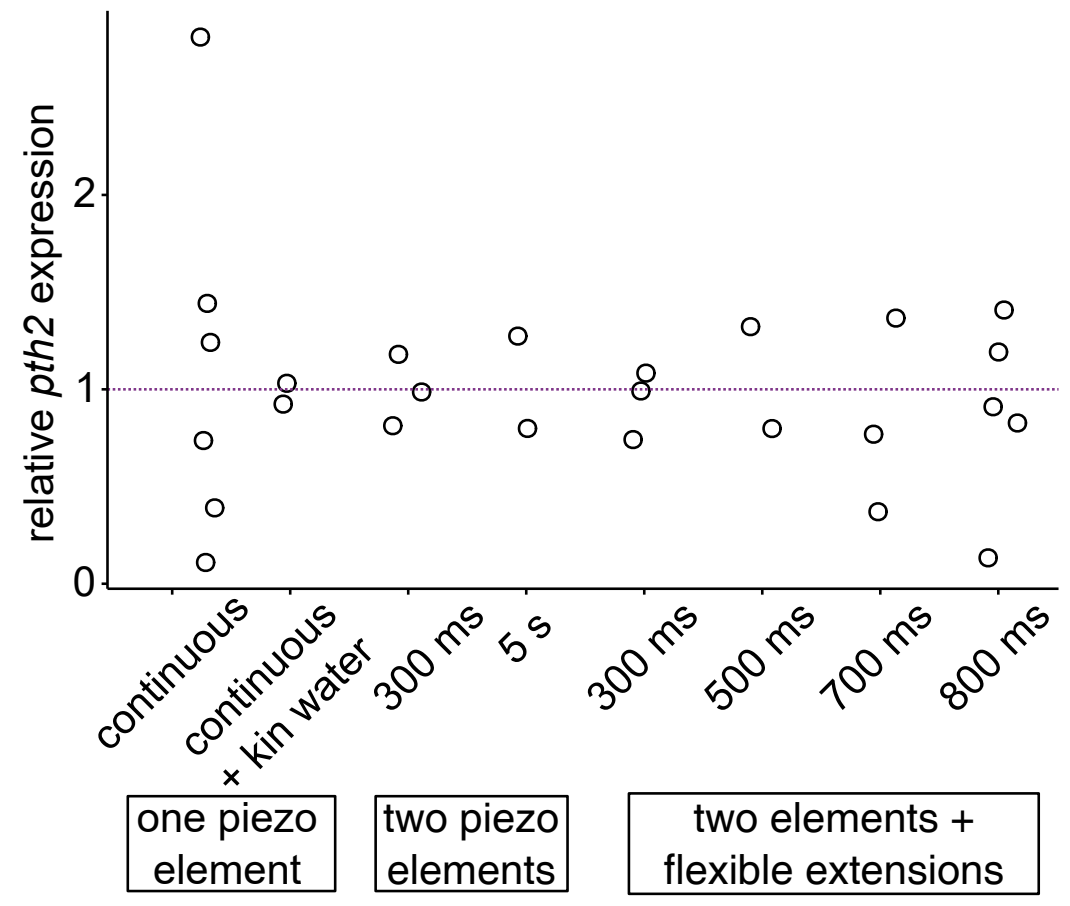

b

\section{C}
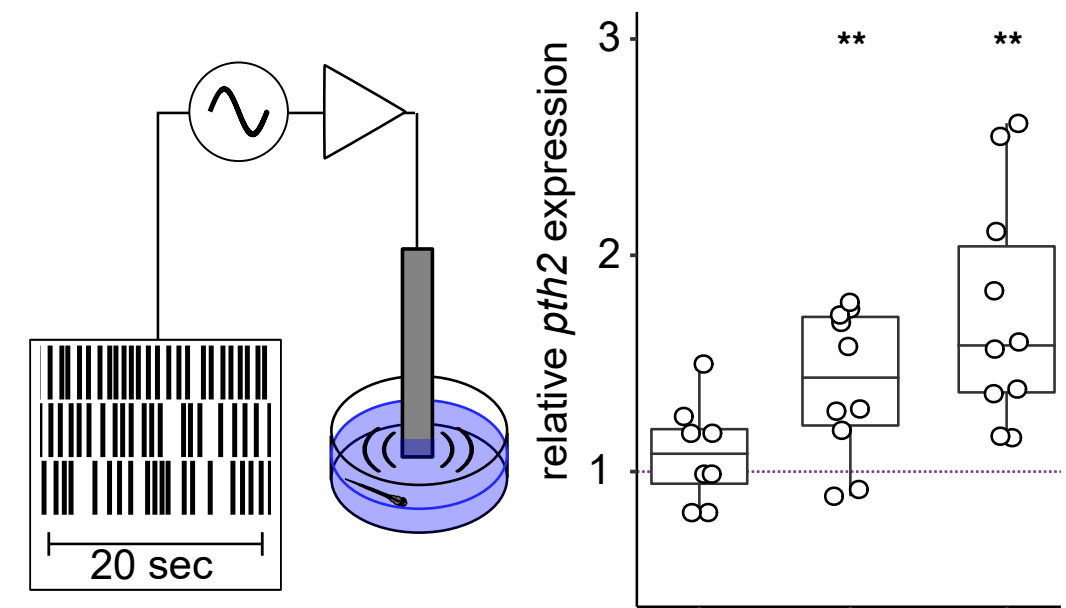

3 hours 3 hours 6 hours
regular variable variable

Figure 3.15: Panel a shows pth2 transcript levels relative to isolated siblings. A piezo actuator was used to deliver mechanical stimulation at $70 \mathrm{~Hz}$ with different inter-stimulus intervals, as indicated on the $\mathbf{x}$-axis. Panel $\mathbf{b}$ is a schematic representation of the piezo device we used and how it delivered stimulation to a zebrafish in a well. The boxplot in $\mathbf{c}$ shows that variable stimulation does increase pth2 abundance. Paired, one-sided t-tests, $\mathrm{BH}$-corrected. For repetitive, regular stimulation of $300 \mathrm{~ms}$ intervals, $\mathrm{p}_{(n=8, t=0.85)}=0.42$, for variable stimulation over three hours, $\mathrm{p}_{(n=10, t=3.76)}<0.01$, for variable stimulation over six hours, $\mathrm{p}_{(n=10, t=5.44)}<0.01$. Adapted after [254]. 


\subsubsection{Modeling larval locomotor features}

To build a model that captured the movement parameters characterizing the fish used in the experiments above, it was necessary to collect high-quality video data that would allow me to extract and reconstruct these locomotion features. Isolated animals (5 dpf) were allowed to explore a dish (diameter of $10 \mathrm{~cm}, 45 \mathrm{ml} \mathrm{E}$ ) while being recorded at a framerate of $20 \mathrm{fps}$. The movement of animals was tracked and their trajectories were used to compute common features such as their locomotion speed. As animals at these early stages move in discrete bouts, a simple peak-detection algorithm could be used to identify periods of acceleration as well as pause periods.

By measuring the time between velocity peaks, the distribution of pause periods can be estimated (resulting in a slight overestimation of actual pause duration). The resulting histogram is right-skewed with a long tail. Different distributions were fitted to the obtained data and their quality of fit measured with the Akaike information criterion (AIC) (table 3.1), with the log-logistic distribution providing the best fit.

\begin{tabular}{|l|l|}
\hline distribution & AIC \\
\hline \hline log-logistic & $7.94 \mathrm{E}+03$ \\
\hline log-normal & $8.91 \mathrm{E}+03$ \\
\hline inverse-gaussian & $9.23 \mathrm{E}+03$ \\
\hline birnbaum-saunders & $9.46 \mathrm{E}+03$ \\
\hline gamma & $1.23 \mathrm{E}+04$ \\
\hline logistic & $1.55 \mathrm{E}+04$ \\
\hline nakagami & $1.79 \mathrm{E}+04$ \\
\hline weibull & $1.80 \mathrm{E}+04$ \\
\hline rayleigh & $1.82 \mathrm{E}+04$ \\
\hline
\end{tabular}

Table 3.1: This table summarizes the different distributions that were fit to the interbout-interval dataset with the corresponding AIC as a quality of fit descriptor. Adapted after [254].

In addition, the duration of the bout period was estimated based on published results and modeled as a normal distribution [266]. Taken together, the swimming profile of a zebrafish can be modeled using repeated drawing from these two distributions. This model 
recapitulates duration of the two different modes (propulsion and pause) in which locomotion takes place. In addition, we utilized published larval tail beat frequency to accurately model the mechanical stimulus its movement provides [265].

Using this model, I revisited the piezo-based stimulation paradigm. Indeed, when stimulation was applied utilizing the model of zebrafish locomotion derived above, an increase in pth2 was observed. In agreement with our results on the dynamics of pth2 expression, a longer exposure to the artifical stimulation of 6 hours resulted in a further increase of pth2 (fig. 3.15 c). This finding in conjunction with the experiments in which the neuromasts were ablated confirmed that pth2 is regulated by mechanosensation via the lateral line and that mechanosensation is sufficient to induce the increase in pth2 transcript levels. 
a

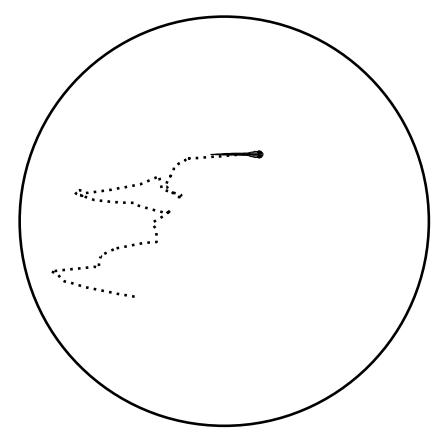

C

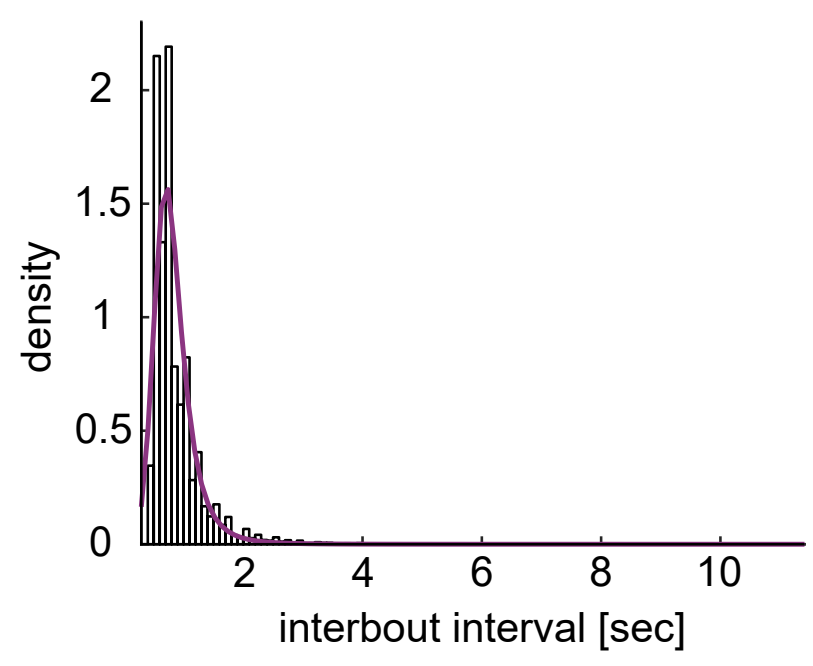

Figure 3.16: To estimate realistic motion features, animals were allowed to freely explore dishes (diameter of $10 \mathrm{~cm}$, a). Their trajectories were reconstructed and used to estimate the inter-bout interval (IBI) distribution (b and c). Adapted after [254]. 


\subsection{Visualization of $p t h 2^{+}$cells}

The experimental findings so far indicate that transcription of the neuropeptide pth2 is regulated by the perception of the movement of other fish. To understand the circuit enabling this, the location of $p t h 2^{+}$cells needed to be identified. To this aim, the following objectives were put forward:

- Visualization of the pth2 transcript by FISH

- Visulization of the pth2 peptide by immunostaining

- Registration of $p t h 2^{+}$cells to a brain atlas

\subsubsection{Creating an a toolset to visualize pth2}

Visualization of mRNA is readily performed using FISH [267, 268]. The neuropeptide pth2 is encoded by a gene of $1.1 \mathrm{~kb}$ length. I amplified an exon-spanning sequence of 564 bp from cDNA and inserted it into a dual-promoter vector, enabling the transcription of RNA probes (fig. 3.17 a).

To visualize the protein translated from pth2 mRNA, an external company (Peptide Specialty Laboratories, Heidelberg) was consulted. An antigenicity analysis, in which a combined score of surface probability and hydrophobicity was used resulted in the identification of two potential antigenic sequences that could be used to create antibodies. An epitope in the sequence of the pro-peptide was used to immunize a guinea pig (fig. 3.17 b). 
a

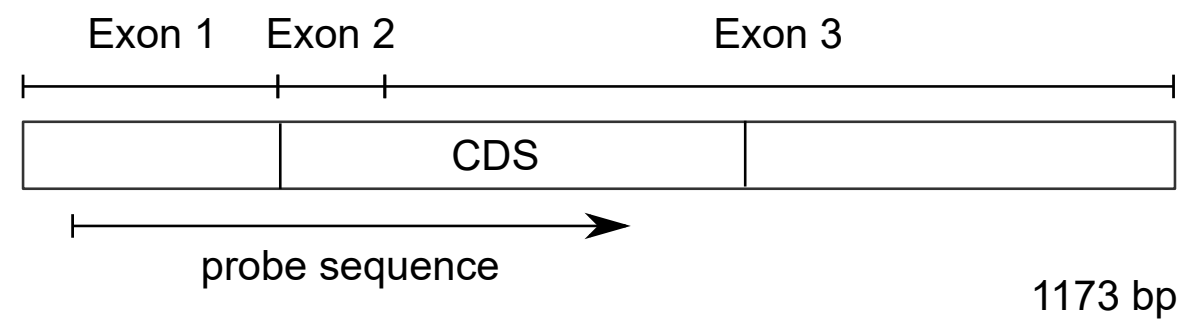

b

\section{MALSLPPRPALLFLVLMSVTLMASAFPQPQLRPLQSNLPA IGQEDSKGEQWEVVYPSISLRDWSIQMLTAPDFGAAKTGR EQLVADDWLPLSQSQMEEELVKGWTGDWPSRVGHQQKR} NIVVADDAAFREKSKLLTAMERQKWLNSYMQKLLVVNSK

Figure 3.17: The pth2 gene consists of three exons, it is schematically depicted in panel a. I designed to probe to overlap the coding sequence as well as the upstream untranslated region. Panel $\mathbf{b}$ shows the aminoacid sequence of the translated pre-pro-peptide. Antigenic sequences are highlighted in purple, the peptide sequence is shown in green. The second antigenic epitope was chosen for immunization and thus antibody synthesis.

\subsubsection{Visualization of $p$ th2}

The labeling of pth2 transcript by [FISH identified a bilateral cluster of cells located in the midbrain of the zebrafish larva (fig. 3.18 a). The mRNA was exclusively localized to the somata, a commonly observation with transcripts of neuropeptides [269]. This very specific localization allowed for straight-forward quantification of the number of pth2 ${ }^{+}$cells in the larval brain. At $5 \mathrm{dpf}$, there are $22 \pm 3.7$ cells visualized in the midbrain by FISH. Immunostainings against pth2 peptide likewise revealed a bilateral population of cells (fig. 3.18b. Signal of both RNA-probe and antibody co-localized, indicating a specific detection of pth2. Visualizing the presence of the pro-peptide by immunostaining showed that pth2 cells project in two major branches:

- an anterior bilateral projection that enters the telencephalon and forms a dense neuropil that connects the hemispheres (fig. 3.18 e). 
- a posterior bilateral projection that at least reaches the level of the posterior part of the optic tectum with few neurites crossing the midline (fig. 3.18 c, d).

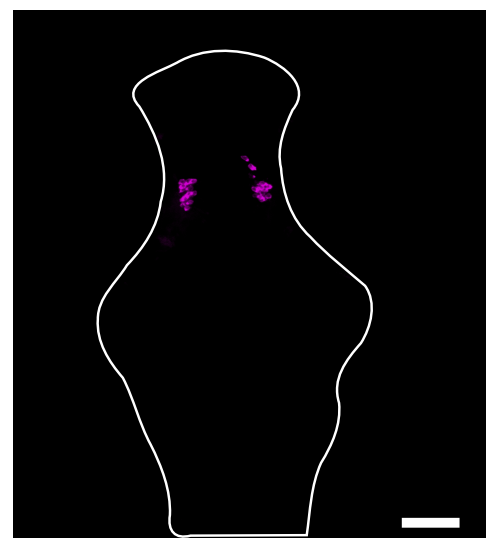

C

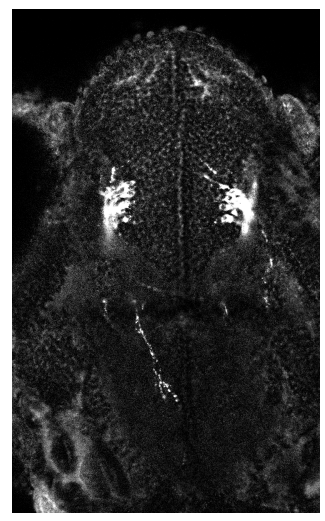

b

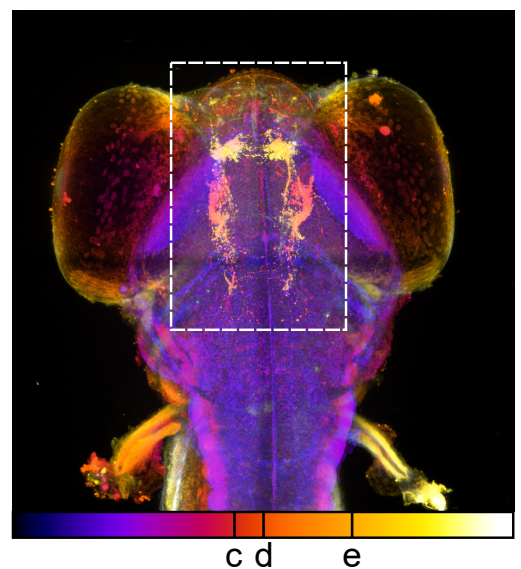

e

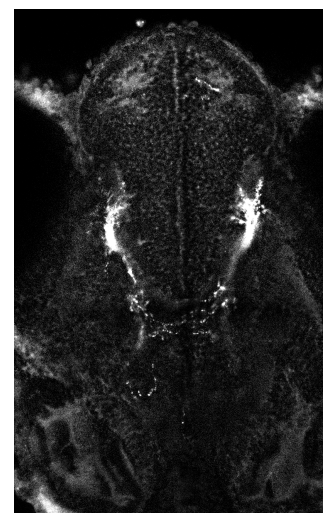

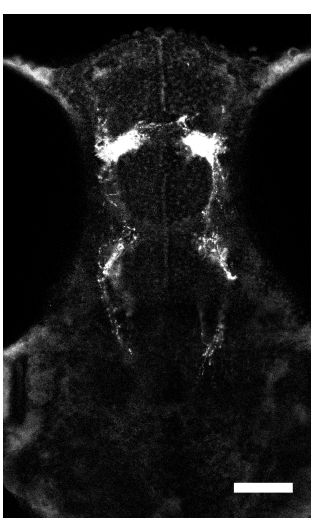

Figure 3.18: In panel a, a maximum-intensity projection (MIP) of a $\mathrm{FISH}$ probe against pth2 is shown, with the brain outlined in white. Transcripts of pth2 are only found in somata, indicative of a very distinct localization. In $\mathbf{b}$, a depth-encoded projection (0 - 184 $\mu \mathrm{m})$ is shown, with single frames at different depths shown in c-e. Scalebar indicates 100 $\mu \mathrm{m}$. Panels $\mathbf{b}-\mathbf{e}$ adapted after [254].

\subsubsection{Registration to the zebrafish brain atlas}

The zebrafish brain has been analysed in great detail and virtual atlases have been constructed that enable the mapping of acquired stacks to a stereotyped standard brain [253]. I performed double-stainings against pth2 (transcript) and synaptotagmin 1/2 znp1, 
which is a commonly used reference stain for registration of brain volumes [?]. The projection of visualized pth2 transcripts on the reference brain allowed to identify brain regions that overlapped the expression pattern. This procedure placed the bilateral cluster of $p t h 2^{+}$ cells in the ventral part of the dorsal thalamus (fig. 3.19). 

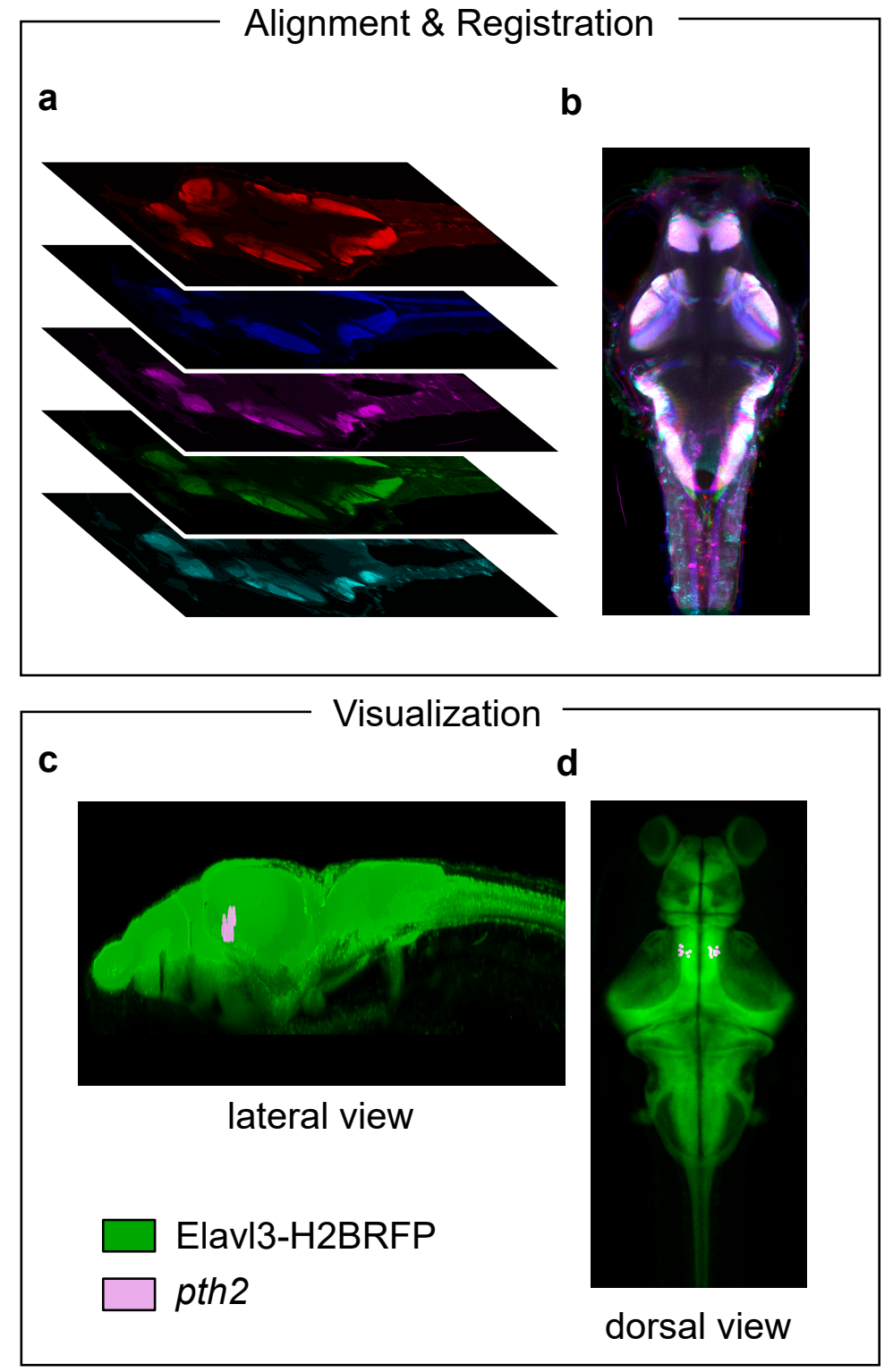

Figure 3.19: For registration of image stacks, a common reference stain was used. In panel a, expression of the reference marker znp1 is shown after alignment of five different stacks. The MIP in $\mathbf{b}$ after registration shows how accurately the brain volumes match after this procedure. Cell somata of $p t h 2^{+}$neurons were manually annotated after registration and displayed by mapping them onto a Elavl3-H2BRFP stack from the z-brain atlas [253], displayed in $\mathbf{c}$ and $\mathbf{d}$. Panel $\mathbf{c}$ and $\mathbf{d}$ adapted after [254]. 


\subsubsection{Characterization of the anatomical environment of $p t h 2^{+}$cells}

Having the tools to visualize pth2-expressing cells allows for a detailed characterization of the cellular neighborhood $p t h 2^{+}$cells are embedded in as well as the investigation of potentially co-expressed molecules. First, I tested whether $p$ th2 ${ }^{+}$cells co-express orthopedia homeobox a (otpa) by visualizing the otpa transcript in combination with an immunostaining against pth2. The transcription factor otpa is an important developmental regulator of neuroendocrine centers in the zebrafish diencephalon [270, 271]. The double-labeling approach showed that $p t h 2^{+}$cell somata are located at the lateral edge of the otpa expression domain and that their anterior projections are following precisely its outline (fig. 3.20 a).

Since otpa is known to regulate the development of dopaminergic clusters I asked whether pth2 $2^{+}$cells might co-express dopamine. To test this, I performed a co-staining against pth2 and the tyrosine hydroxylase protein, the enzyme catalyzing the conversion of tyrosine to L-DOPA, which is the rate-limiting step in the synthesis of catecholamines. Bona-fide dopaminergic cell clusters were located medial to the bilateral $p t h 2^{+}$clusters. No overlapping expression of tyrosine hydroxylase and pth2 was observed (fig. 3.20 b).

In mammals, it has been observed that $p t h 2^{+}$cells project to oxytocinergic neurons [238]. As oxytocin has been implicated in social interactions [122, 272], I asked whether a connection between pth2 ${ }^{+}$and oxytocinergic cells exists in bony fish as well. Oxytocinergic cells were located in a bilateral cluster in the preoptic hypothalamus [273]. Visualizing pth2 and oxytocin peptides in the same animal showed that the oxytocinergic cells were located medially to the pth2 ${ }^{+}$clusters. The anterior projections of the pth2-expressing cells were closely apposed to oxytocinergic cells, but evidence for direct synaptic connections were not observed. 
a

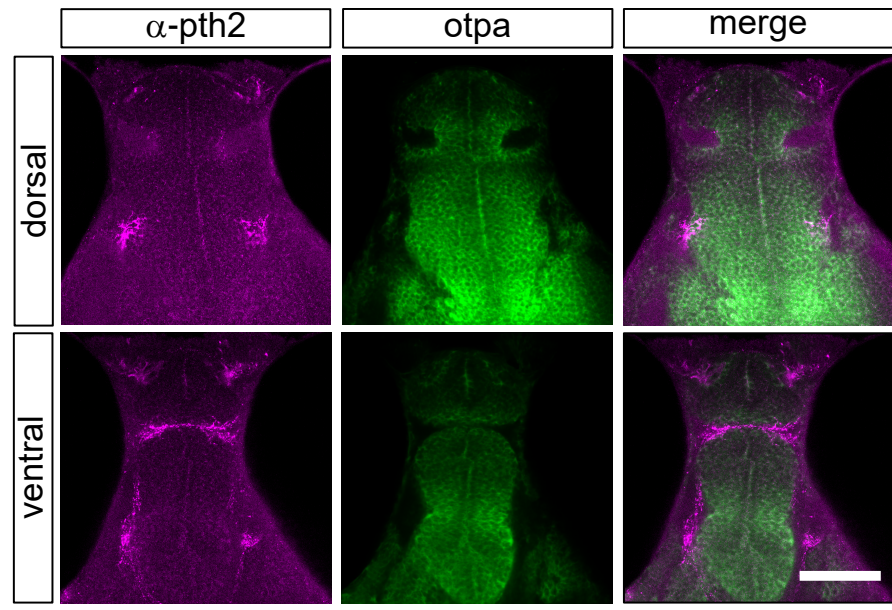

b
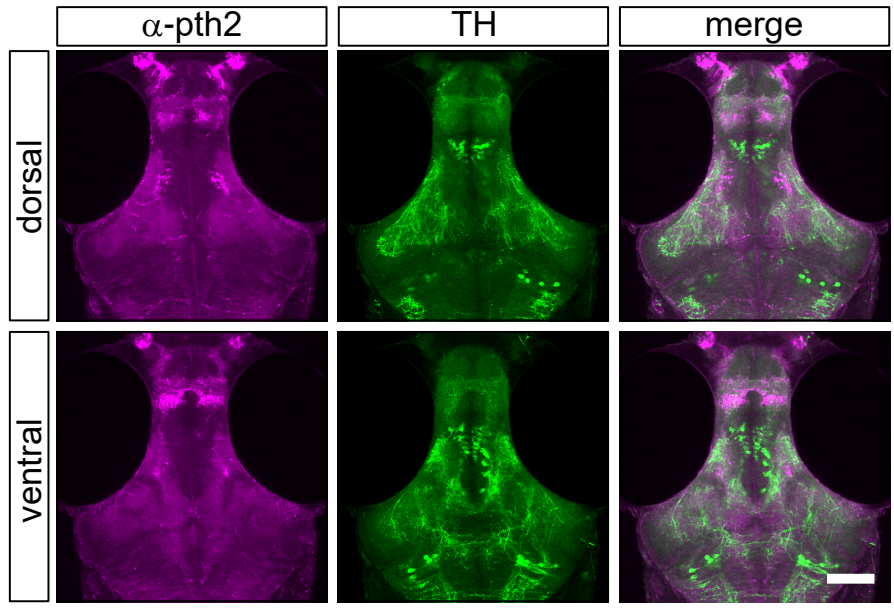

C
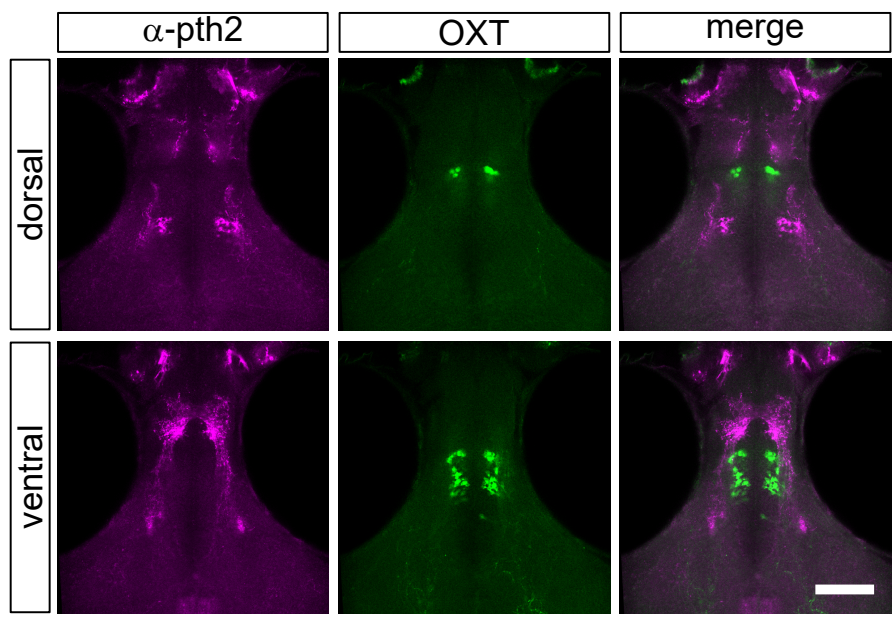

Figure 3.20: This figure shows the overlap between pth2 ${ }^{+}$cell bodies (dorsal) or neurites (ventral) with otpa mRNA (panel a), tyrosine hydroxylase protein (panel b), and oxytocin protein (panel $c$ ). Visualization of pth2 was performed by immunostaining. Scale bars indicate $100 \mu \mathrm{m}$. Adapted after [254]. 


\subsection{Expression of pth2r within the zebrafish juvenile brain}

To identify cell populations potentially influenced by the release of pth2, I used an openly available single-cell sequencing dataset which provided data from the brain of juvenile (23 - $25 \mathrm{dpf}$ ) zebrafish [246]. In this dataset, approximately $9 \%$ of all cells expressed pth2r and the transcript was detected in all identified cell type clusters (see fig. 3.21). In a bootstrap analysis, in which I randomly assigned cells to clusters, I could show that in a large fraction of cell types, pth2 $r$ is at least as strongly expressed as one would expect by chance (see fig. $3.21 \mathrm{c}$ ). These data suggest that pth2 has a broadscale influence and potentially modulates several different brain areas. 
a

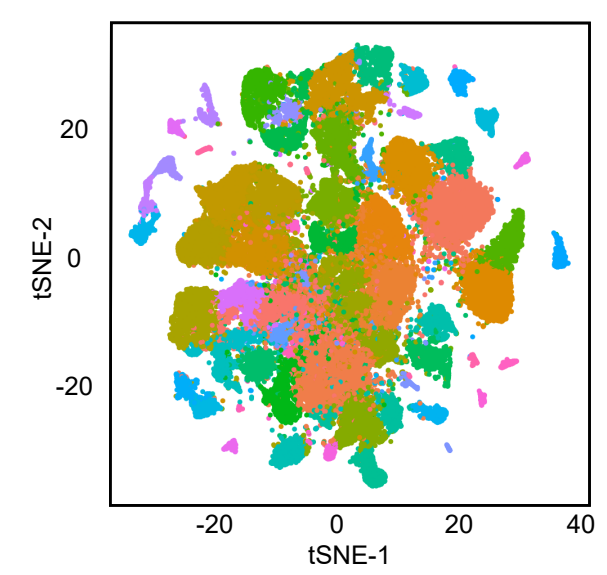

b

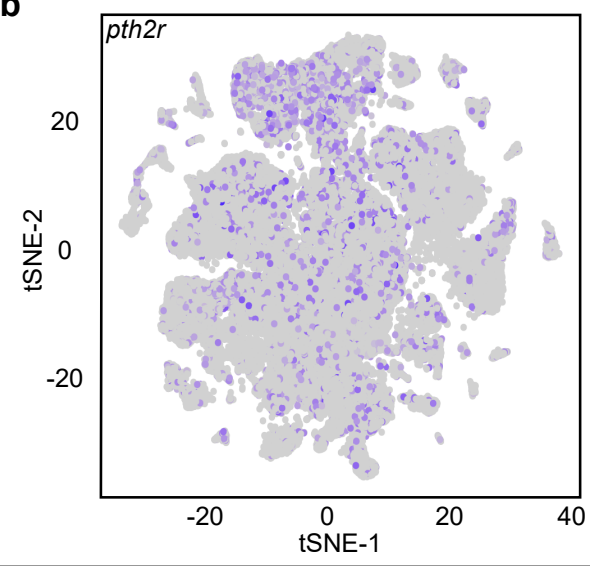

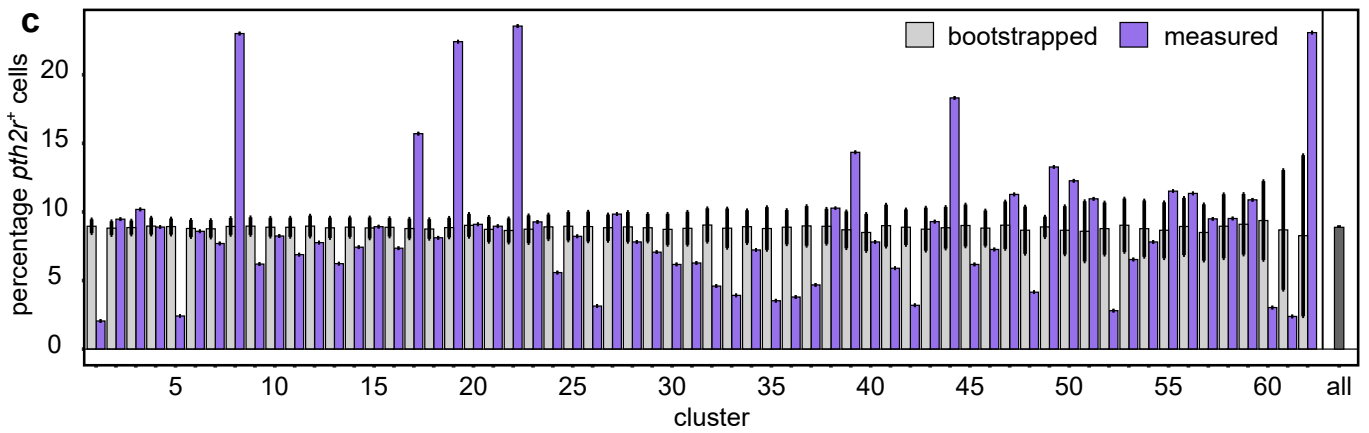

Figure 3.21: The data in this figure were obtained from Gene Expression Omnibus accession number GSE105010 and analysed using the Seurat pipeline. Panel a shows the individual cell types assigned in [246]. In panel $\mathbf{b}$, the expression strength of pth2r is shown, with gray indicating no expression and increasing purple shades indicating higher expression levels. Barplots in c show pth $2 r^{+}$cells for each cluster (measured). Additionally, a bootstrapped estimation indicates the percentage to be expected by random sampling of cells. Lastly, the percentage of $p t h 2 r^{+}$cells across the entire population is shown (all). Adapted after [254].

\subsection{Expression of pth2 $r$ in mouse and human cortical cell}

\section{populations}

As pth2 is found in most vertebrate species, is there a similar expression pattern of pth2r observed in other animals as well? To test this, I utilized the openly available datasets provided by the Allen Brain Institute. In a dataset obtained from mouse cortical and hippocampal cells [248] (approx. 76.000 cells) and a dataset from human cortex [249] (ap- 
prox. 49.000 cells), I analyzed the expression pattern of pth2r (see fig. 3.22). In both cases, the receptor appeared to be mainly expressed in glutamatergic cell populations and its average expession varied from $7.2 \%$ in mouse to $7.9 \%$ in human. 


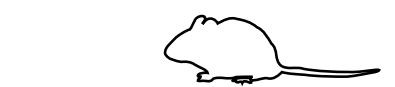

mouse cortex + hippocampus
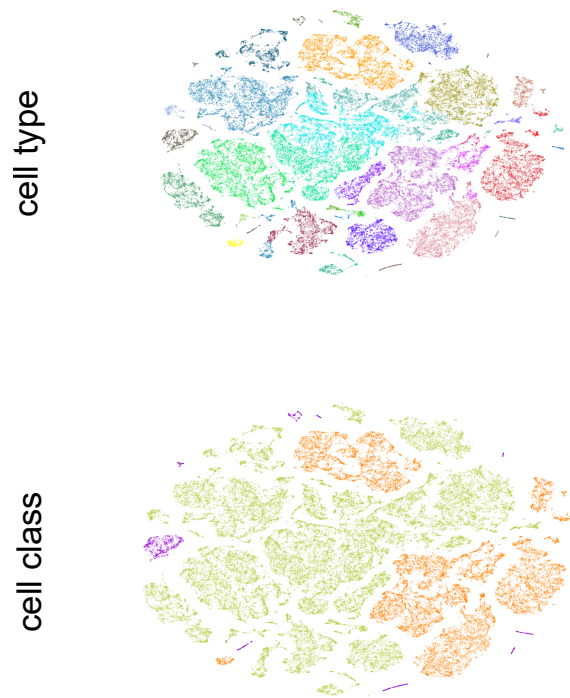

을
0
0
$\frac{0}{0}$
$x$
0
$\grave{N}$
$\frac{1}{2}$

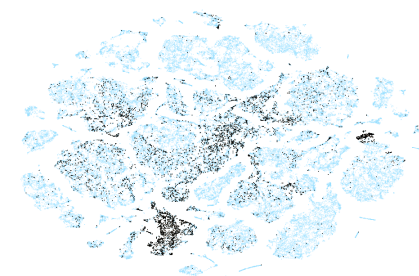

$7.2 \%$

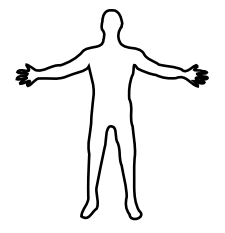

human cortex
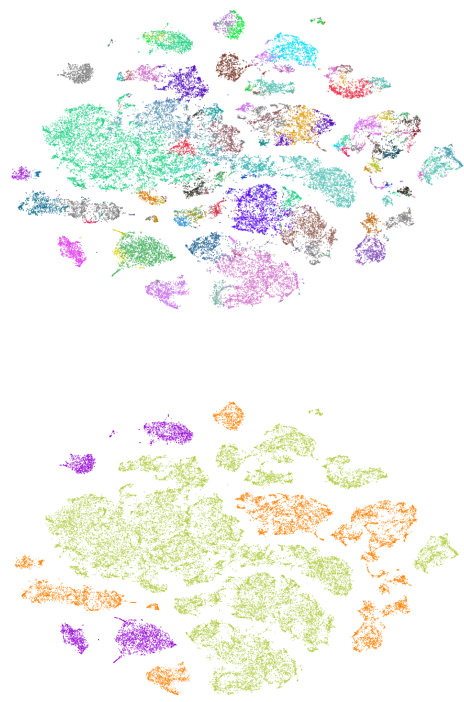

$\square$ glutamatergic $\square$ GABAergic

$\square$ non-neuronal

other pth2r expressed no expression

Figure 3.22: In this figure, expression strength of $p t h 2 r$ is depticted as identified in single-cell RNA sequencing datasets from mouse and human brain areas [248, 249]. In all cases, cells are shown in a tsne-plot as individual points. In the first row (cell types), colors indicate different clusters of cell types as revealed by differential gene expression [248, 249]. The second row (cell class) indicates whether cells are glutamatergic, GABAergic, or belong to a different category. In the last row (pth2r expression), transcript abundance is binarized (black indicating the presence, blue the absence of pth2r). 


\subsection{Contribution of the stress axis to pth2 expression}

As social isolation induces stress in several species [274, 130, 36], it is conceivable that the differential expression of pth2 in different social densities is at least influenced by the stress levels of the animal. To test this, I revisted the initial NGS dataset and compared the expression level of genes that have been implicated in the regulation of stress: steroidogenic acute regulatory protein (StAR), nuclear receptor subfamily 3, group C, member 1 (nr3c1), proopiomelanocortin a (pomca), proopiomelanocortin b (pomcb), arginine-vasopressin (avp), and oxytocin (oxt). None of these genes were differentially regulated when compared between social and isolated conditions (see fig. 3.23 a) and for two of these genes, this result was confirmed using qPCR (fig. 3.23 b). These data suggest that the stress axis is only mildly, if at all, activated by the treatment conditions introduced above. 

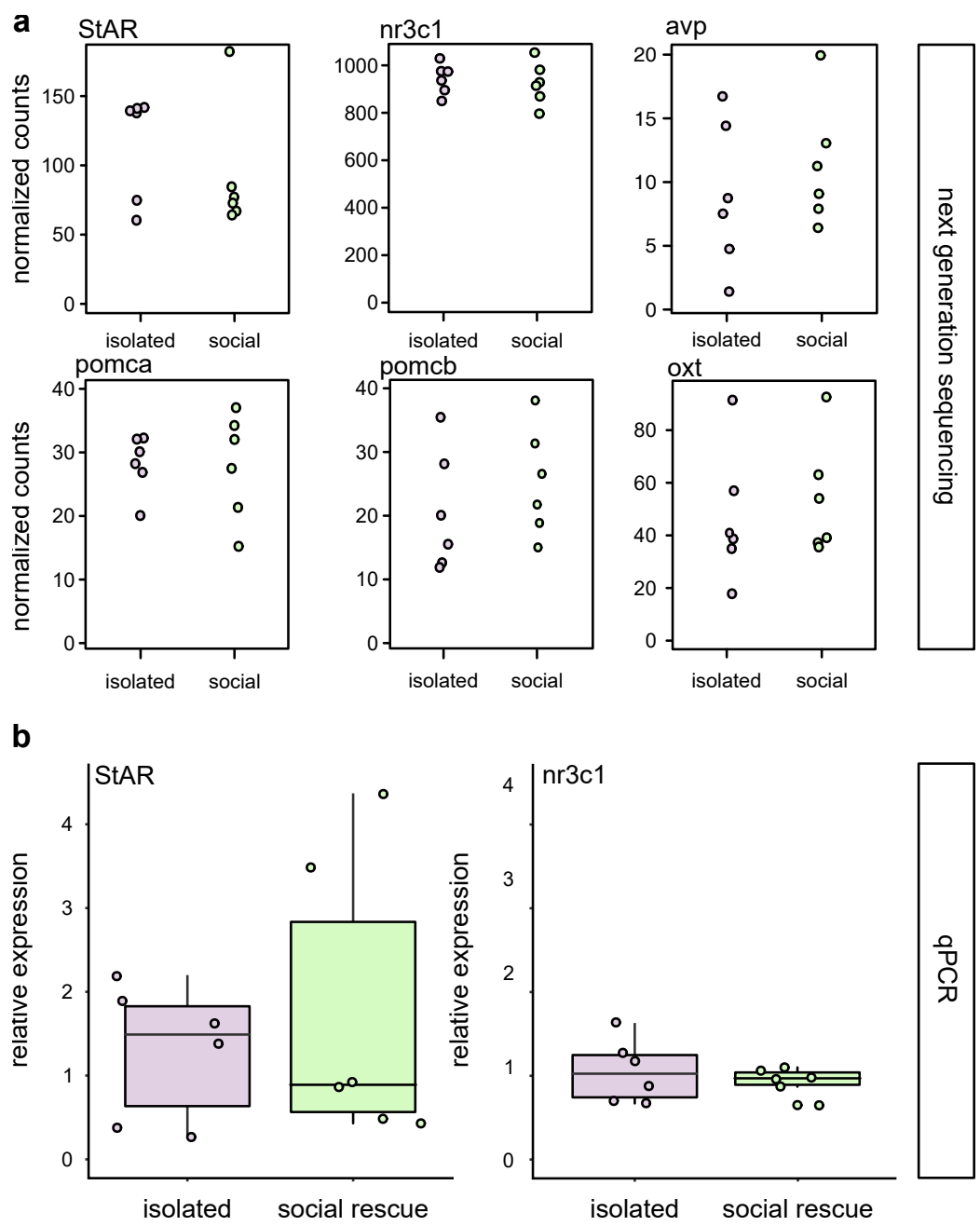

Figure 3.23: In panel a, gene expression data of previously described stress-responsive genes is depicted (see main text for gene details). For all genes, $n=6$, FDR-corrected quasi-likelihood F-test implemented in edgeR, StAR: $p=0.99$, nr3c1: $p=0.99$, avp: $p$ $=0.99$, pomca: 0.99 , pomcb: $p=0.99$, oxt: $p=0.99$ ). Panel $\mathbf{b}$ shows the validation of gene expression by qPCR for StAR and nr3c1 after short introduction of conspecifics to previously isolated fish for 3 hours. Boxplots show expression relative to mean levels of isolated animals. Paired, one-sided t-tests, $n=6$ for both genes. For StAR, $\mathrm{p}_{t=0.37}=0.71$, for nr3c1, $\mathrm{p}_{t=-0.45}=0.66$. Adapted after [254].

\subsection{Phenotypic analysis of loss-of-function mutants}

The neuropeptide pth2 is specifically recognized in the brain by its cognate receptor parathyroid hormone 2 receptor a (pth2ra) [232] and potentially by parathyroid hormone 2 
receptor $b$ (pth2rb) as well [275]. A targeted loss of function in pth2 itself or the downstream receptors should be sufficient to disrupt signaling of this neuropeptide and thus enable us to investigate how animals with or without pth2 behave in different paradigms.

\subsubsection{Identification of loss-of-function mutants}

Both for pth2 and pth2ra, loss-of-function mutants have been identified in a largescale screen by the Stemple group [276]. In both cases, a single substitution leads to a premature stop codon so that the final translation product is truncated. The mutation pth2 $2^{\text {sa23129|T } \rightarrow A}$ is characterized by an amber stop codon replacing amino acid 55 out of 157, the mutation pth2r $r^{s a 11169 \mid C \rightarrow A}$ stops translation at amino acid 176 out of 575 . In both cases, at least around two thirds of the translation product is missing, likely leading to a loss of function. In the behavioral experiments described below, both pth2 $2^{s a 23129 \mid T \rightarrow A}$ and pth2 $r^{s a 11169 \mid C \rightarrow A}$ as well as wildtype animals as control were used.

\subsubsection{Impact of pth2 on locomotion}

In a first approach to characterize whether the loss of pth2 might have an impact on the regular development of the animals, basic locomotion features were assessed. Zebrafish at different developmental stages $(5,14$, and 21 dpf were allowed to freely explore a dish (diameter $10 \mathrm{~cm}$ ) for 10 minutes. All mutant fish were maintained as heterozygous and homozygous animals, so that homozygous mutants could be compared with their homozygous wildtype cousins, ensuring a close genetic relationship. The velocity of animals increased with age, as was expected (fig. 3.24), but no clear difference was observed between homozygous mutants and wildtypes at any developmental stage. Additionally, the animals' propensity to explore the center of the dish was analysed (boldness). In novel contexts, an animal's anxiety can be assessed by looking at the degree of thigmotaxis dis- 
played (i.e. tendency to stay close to the walls in an open field) [184]. This degree was measured using a boldness score that computed the fraction of time the animals spent in the center of the dish. Older wildtype animals were more likely to stay close to the walls than younger larvae, but only in pth $2^{\text {sa23129 }}$ there was a significant increase in boldness at 21 dpf (fig. 3.24 c).

a

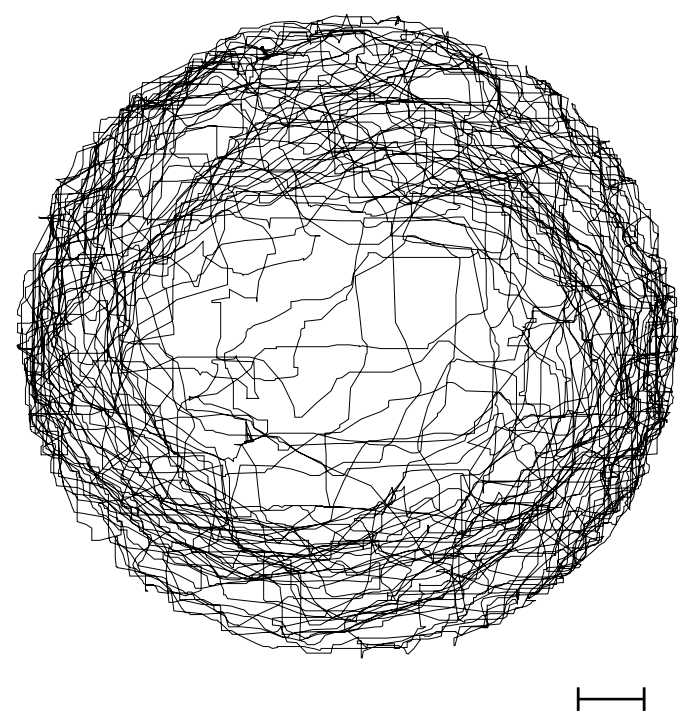

b

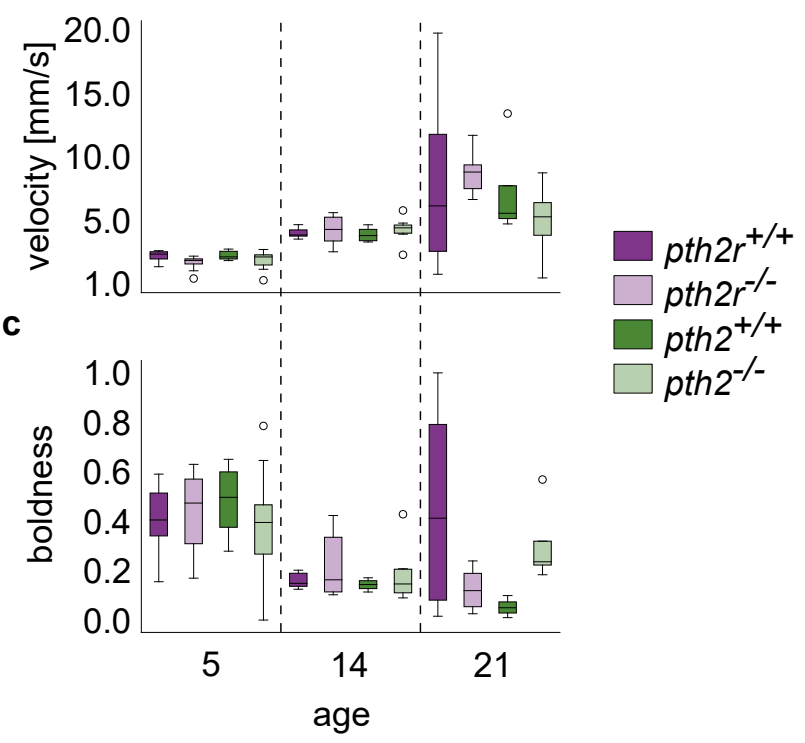

Figure 3.24: Panel a shows an examplary trajectory of a 14 dpf wildtype animal. A clear tendency to stay close to the walls of the open field can be observed. Scalebar $=1 \mathrm{~cm}$. The boxplots in $\mathbf{b}$ show the velocity displayed by animals at different developmental stages and of different genotypes. Number of replicates at $5 \mathrm{dpf}=10$, at $14 \mathrm{dpf}=4-9$, at $21 \mathrm{dpf}=$ $4-7$. A two-way ANOVA showed that only age $\left(\mathrm{p}_{F=76.5}<0.001\right)$ influences velocity, but genotype does not $\left(p_{F=0.63}=0.59\right)$. In panel $\mathbf{c}$, the same trajectories are assessed for boldness. Once again, age was the main variable of interest $\left(\mathrm{p}_{F=22.4}<0.001\right)$. However, an interaction effect of age and genotype was observed $\left(p_{F=3.3}<0.05\right)$, highlighting the fact that pth2 $2^{\text {sa23129 }}$ animals display an increased boldness score at later stages.

\subsubsection{Degree of anxiety as measured by the startle response}

Another approach to measure anxiety levels in animals is the startle paradigm. In this experiment, animals are disrupted by a sudden acoustic or mechanical stimulus, to which they normally respond with a quick escape attempt [277, 59]. In zebrafish (and teleosteans 
in general), this escape response is dependent on a two-component circuit: The Mauthner cells, that induce a fast response to different kinds of stimuli (short-latency C-start (SLC) [61]) and a group of prepontine cells that drive a slower escape response (long-latency C-start ([LLC), [278]). When zebrafish larvae (5 dpf) were exposed to sudden mechanical vibrations, these two escape types became clearly visible by plotting the number of responses over the delay with which they took place after the stimulus onset (fig. 3.25 a). When wildtype animals were raised in different densities (1, 10, or 30 fish per group) until $5 \mathrm{dpf}$ and then tested in this paradigm, no significant differences were found, although with increasing densities, fewer animals tended to respond to stimulation by startling (fig. 3.25 b). This was comcomitant with a non-significant decrease in [LLC whereas the SLC remained unchanged. To assess whether pth2 has an impact on anxiety as measured by the startle resonse, pth2 $2^{s a 23129}$ and $p t h 2 r^{s a 11169}$ animals were raised to $5 \mathrm{dpf}$ in groups of 10 larvae per dish. At this stage, they were subjected to the startle paradigm. The lossof-function mutation in pth2r did not have any effect on the propensity to perform either a SLC, LLC, or to respond to the stimulus at all.

For pth2 $2^{s a 23129}$, animals appeared to be more likely to respond with an LLC (fig. 3.25 c, d) to a startle stimulus. 
a

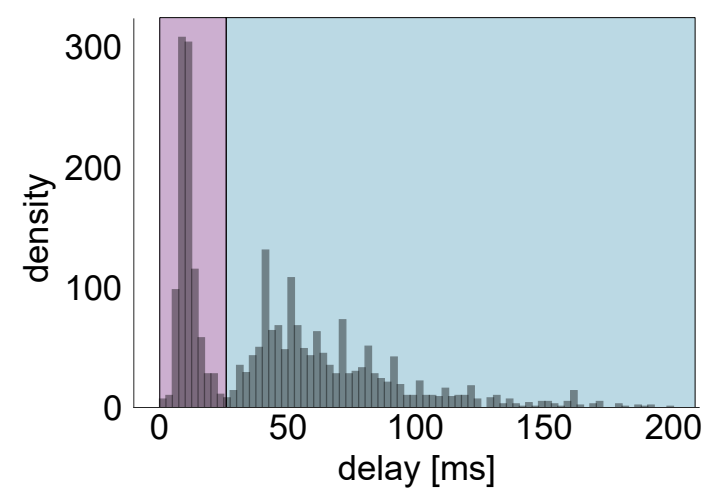

C

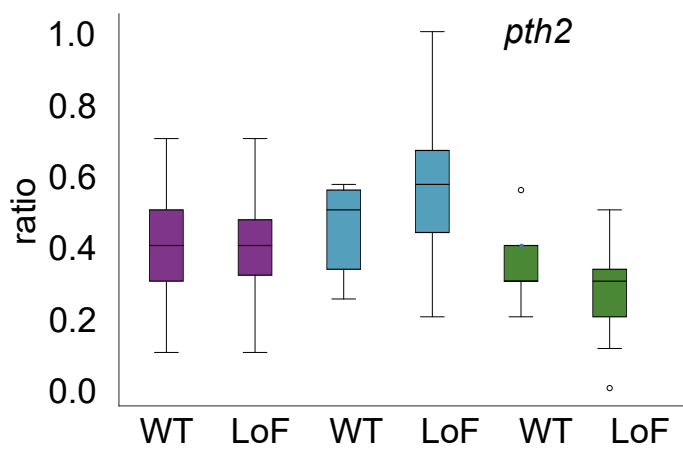

b

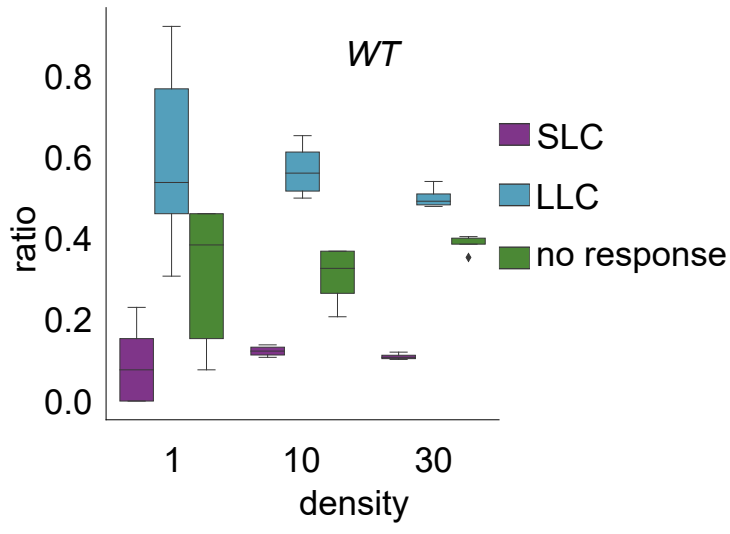

d

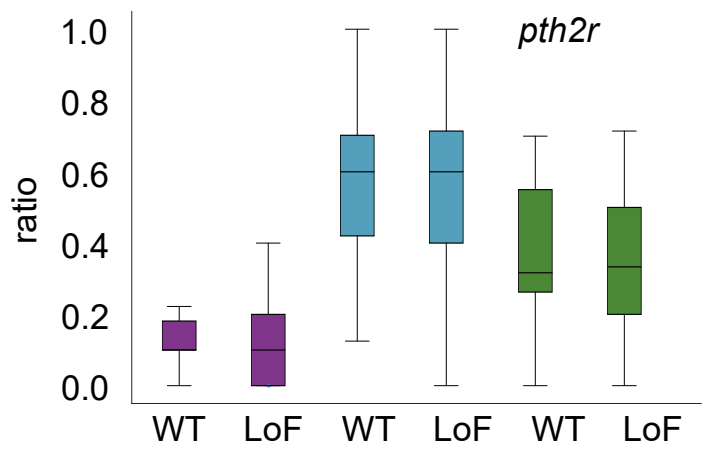

Figure 3.25: Panel a shows response delay times after stimulus onset for 2476 animals. The bimodal distribution is highlighting two different kinds of response types, SLCs and LLCs. The boxplot in $\mathbf{b}$ depicts the impact of raising conditions on response ratio $(n=5$ for density of $1, \mathrm{n}=4$ for other densities). In panel $\mathbf{c}$, responses of pth2 ${ }^{\text {sa23129 }}$ animals in constant densities of 10 fish per experiment are shown $\left(\mathrm{n}_{W T}=13, \mathrm{n}_{\text {sa23129 }}=15\right)$. An increased LLC response ratio is observed in the loss-of-function (LoF) animals $\left(\mathrm{p}_{T=2.1}<0.05\right)$. Panel $\mathbf{d}$ depicts startle responses in pth2 $r^{s a 11169}$ animals $\left(\mathrm{n}_{W T}=22\right.$, $\left.\mathrm{n}_{\text {sa11169 }}=21\right)$. No significant differences were identified.

\subsubsection{Impact of pth2 on social preference in the U-chamber}

As pth2 is strongly influenced by the presence of conspecifics, it is possible that this neuropeptide modulates social interaction. Over development, zebrafish develop a strong tendency to swim towards conspecifics and stay close to them [139]. One approach to measure the extent of this preference is to place animals in a U-shaped chamber with two 
visually accessible adjacent compartments. In one of those chambers, conspecifics are placed, the other remains empty [139]. From the time animals spend in the area close to the "social" compartment, a SPI can be computed, ranging from 0 (no time spent close to conspecifics) to 1 (animals are always close to other fish). At $21 \mathrm{dpf}, p t h 2^{s a 23129}$ and pth2 $r^{\text {sa11169 }}$ fish were placed in the U-chamber for 15 minutes and allowed to explore it freely. Afterwards, conspecifics were added to one of the adjacent compartments. In both cases, the SPI was computed. In all cases, animals displayed a stong tendency to approach conspecifics, and in the case of the mutants, this effect was not different from wildtypes (fig. 3.26).

a

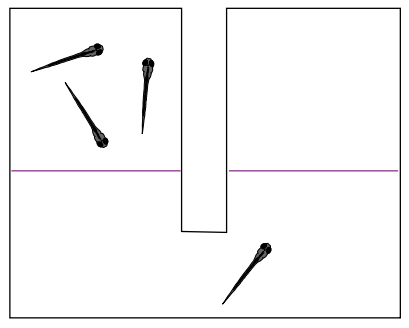

b

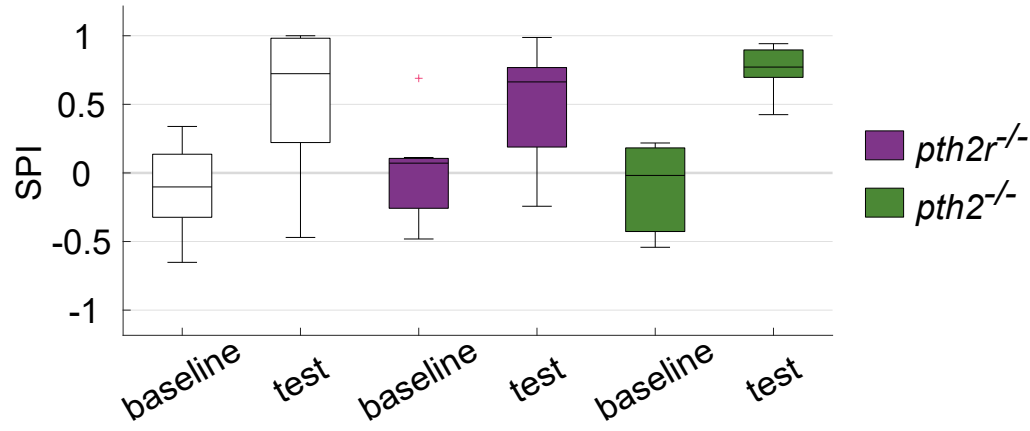

Figure 3.26: Panel a is a schematic representation of the experimental setup. Both arms of the U-shaped chamber are separated from the lower part by a transparent plexiglass barrier (indicated by purple line). The boxplots in $\mathbf{b}$ show the stark increase in SPI when conspecifics were present in one of the adjacent chambers (test as compared to baseline). Number of replicates $=8$.

\subsubsection{Social preference of pth2 mutants in open field}

In the experiment above, animals have to move into one arm of the U-shaped chamber to gain visual access to conspecifics. In another approach to measure social preference without forcing the animals to make this behavioral choice before they even see other fish, 21 dpf zebrafish were placed in an open field with two adjacent chambers, that were 
both visually accessible from every location in the field (fig. 3.27 a). After a baseline exploration of 15 minutes, conspecifics were placed in one of the adjacent chambers and SPI was computed by measuring the time the animals spend in the area that is closer to the conspecifics than to the empty compartment on the other side. In this experiment as well, a strong SPI was observed in wildtype as well as $p t h 2^{\text {sa23129 }}$ and $p t h 2 r^{s a 11169}$ fish and no clear difference between the genotypes was found (fig. 3.27 b).

a

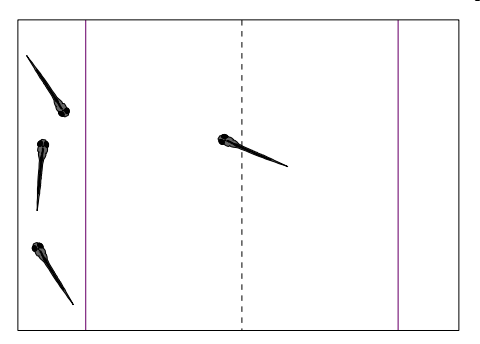

b

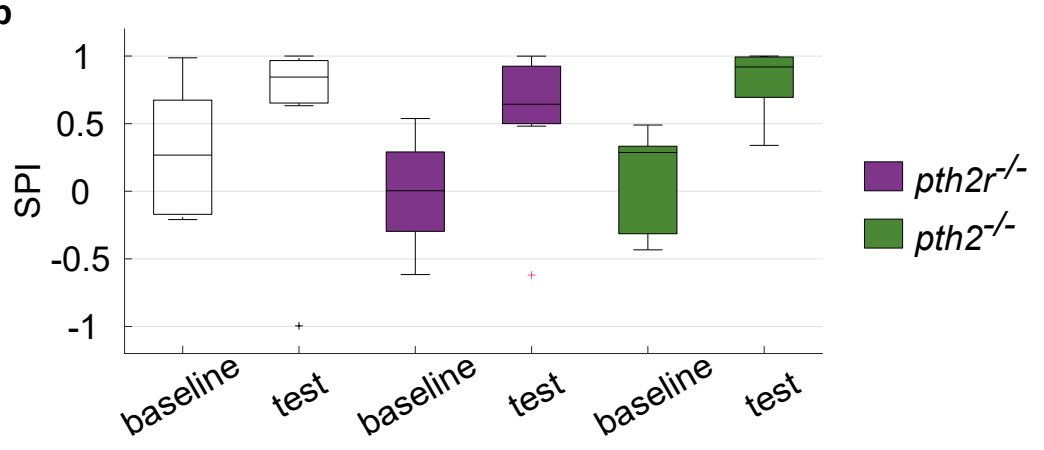

Figure 3.27: In panel a, a schematic of the experimental design is shown. Purple lines indicate transparent plexiglass barriers, the dashed line in the middle of the open field shows how the SPI was computed - if animals moved to the left of the line, this increased the SPI. The boxplot in $\mathbf{b}$ shows the result of the experiment: in all cases, a strong preference towards conspecifics was observed with no clear distinction between genotypes. Number of replicates $=8$. 


\section{Chapter 4}

\section{Discussion}

\subsection{Social regulation of gene expression}

Social animals are highly dependent on the group they live with. This dependency can be observed on the molecular level, and several genes have been reported to be regulated by the social environment [48, 173, 108]. The presence of conspecifics can be thought of as a particular state for the animal, which is contrasted by its opposite, social isolation. However, is it appropriate to conceptualize the wide range of possible social scenarios in a binarized manner? Using this framework, comparing the presence of conspecifics with their absence and how these two states alter an animal's physiology or gene expression will result in the detection of qualitative differences. So far, the impact of social interactions or dominance hierarchies on gene expression was mostly analysed in a qualitative framework, so that e.g. gonadotropin-releasing hormone 1 (GnRH1) becomes upregulated in subordinate cichlids after the removal of dominant males [113] or that the neuropeptide tac2 is strongly expressed in rats after prolonged periods of isolation [48]. Here, we show that the neuropeptide pth2 quantitatively reflects the density of conspecifics in its environment and is consistently downregulated in isolated animals at all developmental stages 
tested. The data presented in this thesis suggest that zebrafish perceive the presence of conspecifics in a fine-grained and nuanced manner. Recent findings in rodents have shown that the presence of conspecifics evokes responses in different neuronal populations, based on the degree of kinship [279] and affiliation [280].

In many animal species, social isolation leads to responses of the stress axis [274, 281, 282]. Increased cortisol levels have been associated with social isolation in primates [88], rodents [89], and fish [90]. In particular, studies in rodents have shown that social isolation can lead to increased levels of CRF [31, 93], one of the molecules mediating glucocorticoid release (see fig. 1.2). This raises the question whether observed transcriptional changes might be explained as part of the animal's stress response. However, the effect of social isolation is far from homogeneous across species, which makes it difficult to draw general conclusions about the effect of social isolation [282]. In zebrafish, social isolation has indeed been shown to even decrease cortisol level in several independent studies [94, 95, 283]. In our own NGS dataset, we did not observe any genes involved with the stress response to be differentially regulated.

Given that many brain areas have been found to be dedicated to particular tasks, it is likely that neural circuits involved with the perception of conspecifics are localized to cartain brain areas. In this study, a global sequencing screen was performed, in which we assessed gene expression in the entire central nervous system. This was a strength yet also limitation of our study: It enabled us to identify pth2 to be differentially expressed based on the social environment. However, a more fine-grained approach might be useful to dissect the impact social isolation has on different brain areas. It has been suggested that social information is processed in designated circuits in vertebrates [284], implicating in particular the preoptic area [109], the anterior and ventromedial hypothalamus [285, 286], the periaqueductal gray [287, 288], the lateral septum [279] and the bed nucleus of the stria terminalis [289] as nodes of a social decision-making network [284], i.e. the network 
responsible for choices regarding the interaction with conspecifics such as mate choice or territory defense. In zebrafish, c-fos stainings after social exposure in previously isolated or socially raised animals provided evidence that some brain areas are more responsive to social cues than others: the preoptic area and the dorsal hypothalamus were identified in this study [110]. Probing gene expression responses in distinct brain areas as opposed to the global approach we used in this study might increase the statistical power to the extent that is necessary to identify genes that become regulated in response to social cues in particular brain regions.

Another natural extension of the work presented here would be the analysis of the three additional genes that were downregulated at all developmental stages analysed. These genes, npas4a, fosab, and egr1 are immediate-early genes and their expression thus signals neuronal depolarization [110]. An approach in which the expression of these genes is mapped to different brain areas by in-situ hybridization might be useful to gain additional insights regarding what brain areas are differentially affected by the presence or absence of conspecifics [110, 253]. In rats, c-fos immunohistochemistry after social isolation has shown that the medial prefrontal cortex, the ventral tegmental area, and the nucleus accumbens shell express lower amounts of this IEG [65]. Another study showed that $c$-fos expression after social isolation is decreased in the basolateral amygdala [290].

\subsection{Information processing of mechanosensory cues and transcriptional regulation of pth2}

This thesis provides evidence that the neuropeptide pth2 is strongly upregulated by the presence of other fish and that this is mediated by the perception of mechanosensory cues. A large body of studies has described in detail how cues perceived by the lateral 
line are processed in the teleostean nervous system (summarized in section 1.3 ) and it is thus of interest to ponder the exact circuit involved. The thalamus, where we identified pth2 ${ }^{+}$cell, receives input from the torus semicircularis, a higher-order processing area of mechanosensory cues [291, 292]. Additionally, the other sensory modalities conveyed by the octaval nerve are processed here in designated areas as well [293]: The nucleus centralis receives auditory information [294, 295], the dorsal part processes electrosensory cues in weakly electric fish [296, 297]. Mechanosensory information impinges on the ventrolateral part of the torus semicircularis [298]. Our finding that only very specific stimulation patterns induce the transcription of $p$ th2 is in line with findings that neurons in the nucleus centralis of the torus semicircularis are specifically tuned to vocalizations of conspecifics [299, 300, 301]. Mechanical cues at particular frequencies have been shown to be important signals for different kinds of behavior in fish: For example, spawning behavior in salmon is elicited and timed by vibrational cues of both fish [302] and these cues are processed by the lateral line [199]. The authors used a vibrating sphere to show that spawning is specifically induced by frequencies of $21 \mathrm{~Hz}$ [199]. In blinded fish, vibrations induce an unconditioned feeding response (i.e. movement towards the source of vibration, followed by the attempt to swallow the source) and these vibrations are most strongly induced at $50 \mathrm{~Hz}$ [303]. These data in conjunction with our findings suggest that the lateral line is capable of using different stimulus properties such as the vibrational frequency and the temporal features of the stimulus and potentially detect particular combinations. This is indicative of feature detectors, i.e. neurons or neuronal networks in higher-order areas of the lateral line circuit that filter sensory stimuli to detect behaviorally meaningful cues [304].

Electrophysiological recordings in the ventrolateral part of the torus semicircularis in combination with stimulation by our established piezo-paradigm might be a useful way to identify those potential feature detecting units [299, 300]. Another approach to find brain 
areas responding to piezo stimulation would be the application of brain-wide imaging of calcium dynamics in combination with piezo stimulation [295, 305]. This would enable the simultaneous interrogation of all areas possibly implicated in the neural processing of lateral line information. Although this second approach lacks in temporal resolution as compared to electrophysiological recordings, it makes it possible to look at the processing of mechanosensory cues in an unbiased manner while maintaining the biological integrity of the animal.

Another question is how the detection of specific mechanosensory cues is then used to trigger the transcription of pth2 in thalamic cells. For several neuropeptides, secretion and synthesis are coupled, which means that there is a common mechanism triggering both the release of neuropeptides from dense-core vesicles and and their synthesis in order to restore peptide levels [306]. The release of dense-core vesicle content is controlled by proteins modulated by calcium levels such as CamKII, which in turn phosphorylate and thus activate the ryanodine receptor, further facilitating calcium release from the endoplasmic reticulum [307, 308]. In addition, voltage-induced increase of intracellular $\mathrm{Ca}^{2+}$ levels have been shown to not only cause secretion [309] but also trigger the transcription of the corresponding neuropeptide genes as well [310, 311]. This prevalent coupling of secretion and synthesis that is observed in neuropeptides would suggest that the excitation of the pth2 ${ }^{+}$ neurons by feature-detecting cells might be sufficient to trigger transcription. A detailed analysis of the molecular cascade involved will be necessary to identify the driver of transcription and a differential chromatin immunoprecipitation analysis of the upstream region of pth2 in isolated and social animals would be a suitable tool to identify the transcription factors involved. However, based on the extensive work performed on the lateral line, a proposed minimum circuit necessary to propagate information from the neuromasts to the pth2 ${ }^{+}$cells in the thalamus would consist of the following connections: Activated neuromasts release glutamate, inducing graded potentials in the afferent fibers of the anterior 
or posterior lateral line [189]. Both converge on the medial octavolateral nucleus in the hindbrain, which collects mechanosensory and auditory information [196]. This nucleus projects to the torus semicircularis in the midbrain, which itself has been described to be connected to the thalamus [196], where we showed the pth2 ${ }^{+}$cells (see figure 4.1). This hypothesized circuit could now be tested in detail with the methods outlined above.

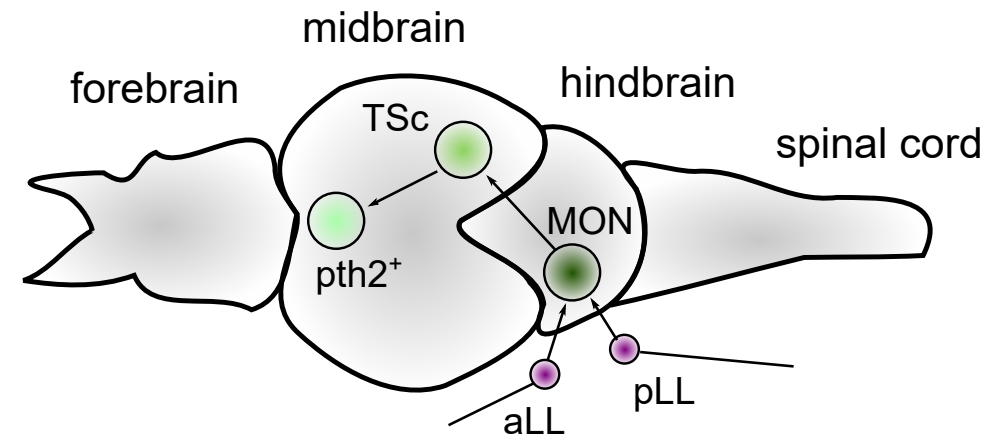

Figure 4.1: This cartoon illustrates a potential minimum path for the propagation of sensory information from the lateral line to the $p t h 2^{+}$cells in the thalamus. Abbreviations: aLL - anterior lateral line, pLL - posterior lateral line, MON - medial octavolateral nucleus, TSc - torus semicircularis.

\subsection{Mechanosensory perception of social cues}

Our results suggest that some social cues in zebrafish are perceived via the lateral line, which receives mechanosensory cues from the surrounding water. In other systems, mechanosensation has been found to play an important role in the transmission of social information. For example, cichlid males have been observed to gauge an opponent's size before engaging in an actual fight for dominance. They do this by performing aggressive lateral displays, during which they suddenly move their body parallel to the body axis of their potential opponent, thus creating a turbulence in the water that is proportional to their body's size. Under normal circumstances, these lateral displays are sufficient to prevent a fight if one of the males is significantly larger than the other. However, if the lateral line 
is chemically ablated, assessment of the opponent before a fight takes about 10 times as long and, once the fish physically engage, cichlids whose lateral line was ablated rely more on contact behavior such as biting or ramming of their opponent [188, 312].

Another example of how mechanical cues inform animals about their social environment is found in locusts. These animals either exist in a solitary state, when the number of locusts in an area is low, but can enter a gregarious state if the density of conspecifics becomes too high [20]. This switch is triggered by the specific stimulation of an animal's hindlegs, leading to the formation of locust swarms [313, 314]. Our data further supports the notion that social cues can be transmitted mechanically. It remains to be determined how complex the representations can be that are derived from this kind of input.

In the context of social interaction, work on mechanical cues transferring social information is relatively sparse. Most research focuses on visual or olfactory information [139, 315]. One possible reason for this becomes clear in the particular case of fish and other aquatic animals: Mechanical stimuli lead to complex water movements that are computationally expensive to model and understand [316, 317, 318]. It is thus very challenging to recapitulate salient features of e.g. conspecific locomotion in a precise manner. In the piezo paradigm introduced in this thesis, pth2 levels were increased approximately 1.4 -fold as compared to baseline levels of isolated animals after 3 hours of stimulation (see fig. 3.15). The model we fit our data to (from actual conspecifics - see fig. 3.7) would predict an upregulation of about 1.9-fold, suggesting that we have not recapitulated all relevant features in our approach sufficiently to perfectly mimic the presence of conspecifics. One obvious difference is the static location of the piezo and its orientation - while actual conspecifics move around and beat their tails in a horizontal plane, the piezo actuator delivers stimulation from above and remains at the same place. Nevertheless, the fact that this stimulation was sufficient to induce pth2 transcription demonstrates quite clearly that at least a necessary part of the features recognized by the system was recapitulated. 
It is interesting to consider the discovered system in an evolutionary framework. The data presented in this thesis suggest that pth2 is not regulated by any other modality than mechanosensation. This is in contrast with data from rodents, which show that projections from the auditory cortex and the superior colliculus reach pth2 ${ }^{+}$cells [225] as well. It is conceivable that over evolutionary time the circuit became modified and received additional inputs from other modalities. Additionally, in zebrafish we only identified one population of pth2 ${ }^{+}$cells, whereas in rodents, three different groups have been identified [225] (see fig. 1.6. To understand the evolution of this system and the way it changed would require the investigation of $p t h 2^{+}$cells in representative vertebrate clades. A particularly interesting first approach would be the analysis of anurans, as these animals posses a lateral line as well but can also spend time outside the water for limited amounts of time. Does the presence of conspecifics e.g. in tadpoles lead to an increased transcription of pth2? Is the system shut off while animals are outside the water or are there already compensatory projections in place that inform the animal about the presence of conspecifics?

\subsection{Modulatory effects of pth2}

In other model systems, pth2 has been shown to be involved in specific forms of social interaction. The presence of pups induced depolarization of cells in the $\mathrm{PIL}$ as indicated by increased expression of $c$-fos. The vast majority of these cells expressed pth2 [238]. It was shown that specifically suckling of the pups led to a strong increase in pth2 levels [239]. Other studies demonstrated that projections from pth2 ${ }^{+}$cells reach oxytocinergic [218, 319] and arginine-vasopressin-positive cells [320], cell-types that have been implicated in the regulation of social behaviour in several studies across a wide variety of model organisms [321, 272]. It is thus likely that the rapid regulation of pth2 by changes in the social environment that is reported in this thesis might be linked to social behavior as well. 
It can be excluded that pth2 regulates maternal care in zebrafish, too, as this species does not display any parental care. Several aspects of social interaction were tested and reported in this thesis using animals with a nonsense mutation in the pth2 and pth2r gene. No difference was observed in the social preference that animals display towards conspecifics (see figs. 3.26 and 3.27). It will be worthwhile to explore social interaction in a more in-depth manner by going beyond simple preference tests and give animals the opportunity to freely explore their surroundings together at later stages of development. Latestage juvenile and adult fish display rich social interaction such as schooling and shoaling [140, 171] and the way animals interact in a novel environment allows for the analysis fo several parameters of interest, such as the general features of the shoal such as cohesiveness or polarization [18], which can also be interpreted as indications of anxiety.

It is in fact quite relevant that in the startle paradigm we applied in this work to analyse the impact of the presence or absence of pth2, we observed an appreciable difference between wildtype and mutant animals in our behavioral tests (see fig. 3.25). Several studies implicate pth2 in the regulation of anxiety-like behaviors [234, 322]. Social isolation has been shown to increase states of anxiety and fear [40, 65, 323] and produce a prepulse inhibition deficit in startle paradigms [63, 324]. Further analysis of anxiety-related behaviors in zebrafish such as prepulse inhibition, light preference [325], or fear memory might be useful to gain additional insight in the way pth2 is potentially modulating behavior.

To appreciate the effect of pth2, it will be necessary to modulate its abundance in a controlled manner and analyse the neural dynamics of $p t h 2^{+}$cells in different scenarios. Worthwhile paths to explore to this aim would include the generation of transgenic lines targeting pth2+ cells e.g. via CRISPR-Cas9-based approaches [326] or the injection of a construct containing the pth2 open reading frame under the control of an externally controlled element such as the heatshock promoter [327]. The external application of synthesized peptide could be a useful approach, but it needs to be validated whether the 
peptide can enter the brain or needs to be injected. Likewise, it remains to be validated how long the peptide would be active in the brain [328].

\subsection{Summary}

In this thesis, I introduced experimental evidence that the neuropeptide pth2 is regulated by the social environment in zebrafish. Its transcription is quickly increased or decreased in the presence or absence of conspecifics. Neither visual nor chemical cues from conspecifics are sufficient to trigger transcription, but the mechanosensory perception of others via the lateral line was shown to be necessary for this effect to take place. In an approach to model the mechanical cues perceived by the animal, the movement of larval zebrafish was mimicked by a piezo actuator and while many different stimulation paradigms failed to trigger the transcription of pth2, the precise modeling of larval locomotion induced a significant upregulation of pth2. Cells expressing pth2 are found in a bilateral cluster in the thalamus of zebrafish at the edge of the otpa ${ }^{+}$expression domain. First experiments were conducted with animals in which a nonsense mutation either in the gene of pth2 or pth2r prevents gene products to be functional. In the analysis of these fish, a slight effect was observed in pth2 ${ }^{-/-}$animals in the startle paradigm, in which more animals displayed a LLC potentially indicating an effect of pth2 on the regulation of anxiety states. Future directions have been described in the discussion above, providing examples of how this socially modulated neuropeptidergic system can be explored in more detail. 


\section{Appendix A}

\section{Computer Code}

The following Matlab code was used to trigger the piezo and induce a stimulation pattern that was similar to the actual movement of zebrafish larvae.

\% clear workspace

clear all

$\%$ create interbout-interval and bout length distribution:

pd_ibi = makedist('Loglogistic', 'mu', -0.27, 'sigma', 0.21);

pd_bout $=$ makedist ('Normal', $0.15,0.1)$;

$\%$ create arduino object

$\mathrm{a}=\operatorname{arduino}$

a. configurePin ('D9', 'Unset');

a. configurePin('D9', 'DigitalOutput');

$\%$ pre-specify the random number generator and create lists

$\%$ to store interbout-intervals and bouts 
rng ('default');

$|B| \_$dist $=[]$;

bout_dist $=[]$

$\%$ track time

totalTime $=0$;

boutProfile $=\left[\begin{array}{ll}0 & 1\end{array}\right]$;

while totalTime $<(3.1 * 3600) \%$ will run for at least 3.1 hours bout $=\operatorname{abs}\left(\right.$ random $\left.\left(p d \_b o u t\right)\right)$;

a. writeDigitalPin ('D9', 1);

pause (bout);

totalTime $=$ totalTime + bout $;$

boutProfile = [ boutProfile; totalTime 0];

a. write DigitalPin ('D9',0);

$i b i=\operatorname{abs}\left(\operatorname{random}\left(p d_{-} i b i\right)\right)$;

pause (ibi);

$|B|_{-}$dist $=\left[|B|_{-} d i s t, i b i\right] ;$

bout_dist $=$ [bout_dist, bout $]$;

totalTime $=$ totalTime + ibi

boutProfile = [ boutProfile; totalTime 1];

end 
clear a 


\section{Appendix B}

\section{Differentially Expressed Genes}

\section{B.1 Differentially expressed genes at $5 \mathrm{dpf}$}

The following table displays all genes that were identified to be differentially expressed in our NGS experiment at $5 \mathrm{dpf}$. 


\begin{tabular}{|c|c|c|c|c|c|c|c|c|c|c|c|c|c|c|c|c|}
\hline 愛 & 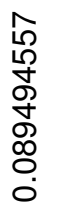 & 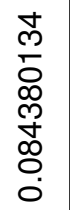 & 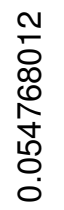 & $\begin{array}{l}\text { న్ } \\
\text { న } \\
\frac{1}{5} \\
\stackrel{0}{0} \\
0 \\
0\end{array}$ & 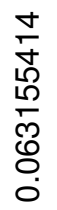 & 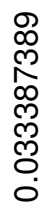 & 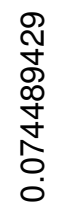 & 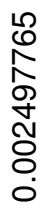 & $\begin{array}{l}m \\
\infty \\
\infty \\
0 \\
0 \\
م \\
0 \\
0 \\
0 \\
0\end{array}$ & 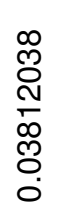 & 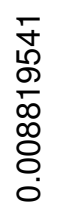 & 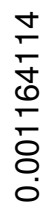 & 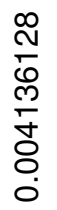 & 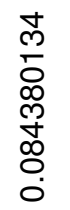 & 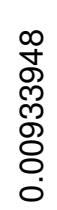 & $\begin{array}{l}\text { N } \\
\stackrel{N}{N} \\
\bar{N} \\
\text { O্ } \\
0\end{array}$ \\
\hline$\frac{\text { D }}{\frac{D}{\pi}}$ & 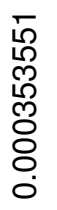 & 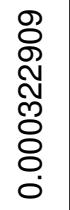 & 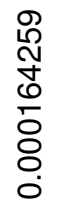 & 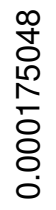 & 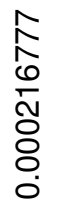 & 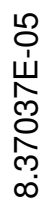 & $\begin{array}{l}\bar{N} \\
\text { N } \\
\text { Nิ } \\
8 \\
8 \\
0\end{array}$ & 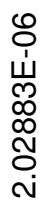 & 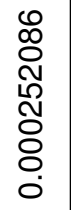 & 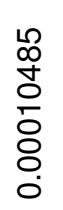 & 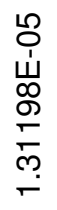 & 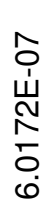 & 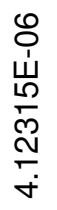 & 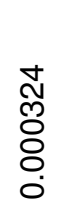 & $\begin{array}{l}\stackrel{2}{0} \\
\text { 山े } \\
\stackrel{2}{0} \\
\infty \\
+ \\
+ \\
+\end{array}$ & 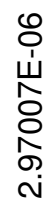 \\
\hline $\begin{array}{l}\sum_{0} \\
0 \\
\text { 음 }\end{array}$ & 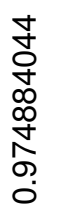 & 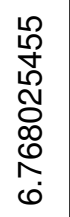 & 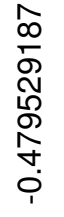 & $\begin{array}{l}= \\
\bar{\sigma} \\
0 \\
0 \\
80 \\
0 \\
\stackrel{0}{N} \\
\text { i }\end{array}$ & 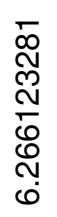 & 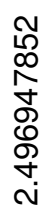 & 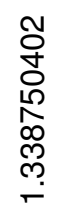 & 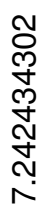 & 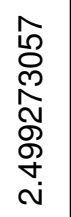 & 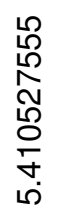 & 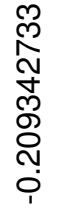 & 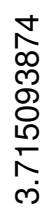 & 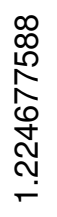 & 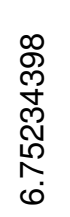 & 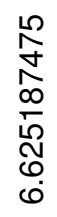 & 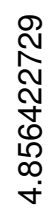 \\
\hline $\begin{array}{l}0 \\
\text { 능 } \\
\underline{0}\end{array}$ & 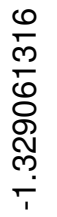 & 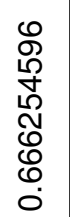 & 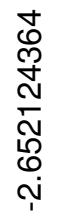 & 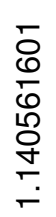 & 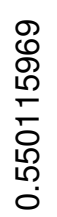 & 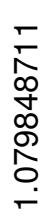 & 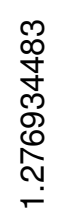 & $\begin{array}{l}\text { o } \\
\infty \\
\infty \\
\infty \\
\infty \\
\infty \\
\infty \\
\infty \\
0 \\
0 \\
0\end{array}$ & 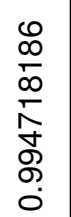 & 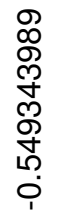 & 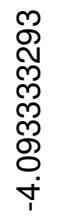 & 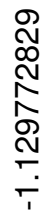 & 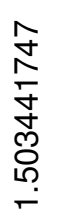 & 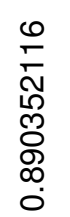 & 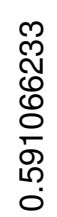 & $\begin{array}{l}0 \\
\infty \\
\infty \\
\infty \\
\infty \\
10 \\
0 \\
0 \\
0\end{array}$ \\
\hline 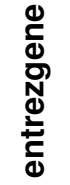 & $\Sigma$ & $\begin{array}{l}\frac{1}{0} \\
\frac{\sigma}{N}\end{array}$ & $\Sigma$ & $\begin{array}{l}\text { ले } \\
\text { ర్ } \\
\text { ઠे } \\
\text { ঃ }\end{array}$ & $\Sigma$ & 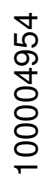 & $\underset{\substack{\stackrel{\infty}{N} \\
\stackrel{\infty}{N}}}{ }$ & 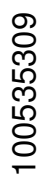 & 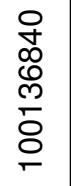 & 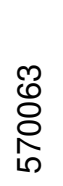 & 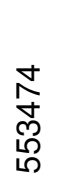 & $\begin{array}{l}\bar{\Gamma} \\
\text { ల్ల } \\
\text { }\end{array}$ & $\begin{array}{l}\infty \\
\bar{\emptyset} \\
\varnothing \\
\llcorner\end{array}$ & $\mathbb{Z}$ & $\begin{array}{l}\mathbb{8} \\
0 \\
0 \\
10 \\
0 \\
10\end{array}$ & 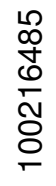 \\
\hline 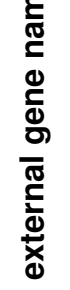 & 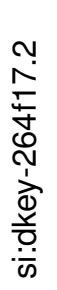 & 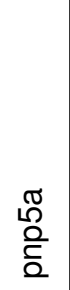 & 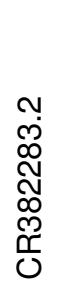 & 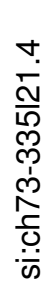 & 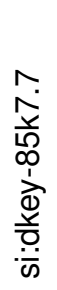 & $\begin{array}{l}\frac{M}{N} \\
\stackrel{2}{0} \\
\frac{2}{2}\end{array}$ & $\begin{array}{l}\frac{d}{N} \\
\frac{1}{0} \\
\frac{2}{2}\end{array}$ & "্তু & 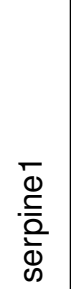 & $\begin{array}{l}\frac{0}{2} \\
\stackrel{0}{0} \\
3\end{array}$ & $\begin{array}{l}\stackrel{x}{0} \\
\frac{8}{2} \\
\frac{\pi}{0}\end{array}$ & $\begin{array}{l}\frac{\bar{E}}{\frac{1}{0}} \\
\frac{\mathbf{d}}{Q}\end{array}$ & 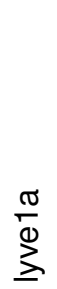 & 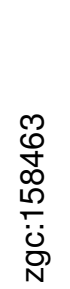 & $\frac{\text { 昰 }}{x}$ & $\begin{array}{l}\bar{\tau} \\
\text { O } \\
\bar{Q}\end{array}$ \\
\hline 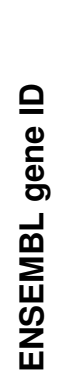 & 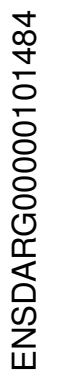 & 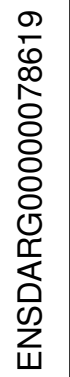 & 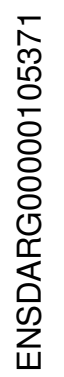 & 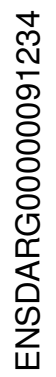 & 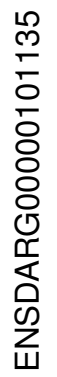 & 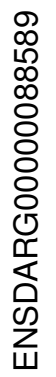 & 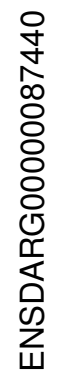 & 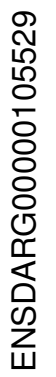 & 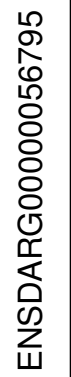 & 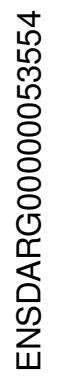 & 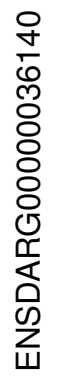 & 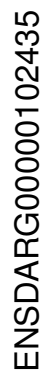 & 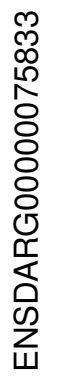 & 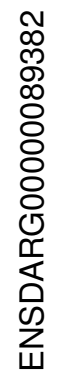 & 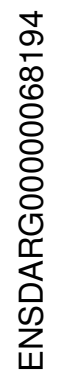 & 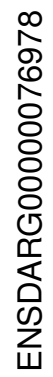 \\
\hline
\end{tabular}




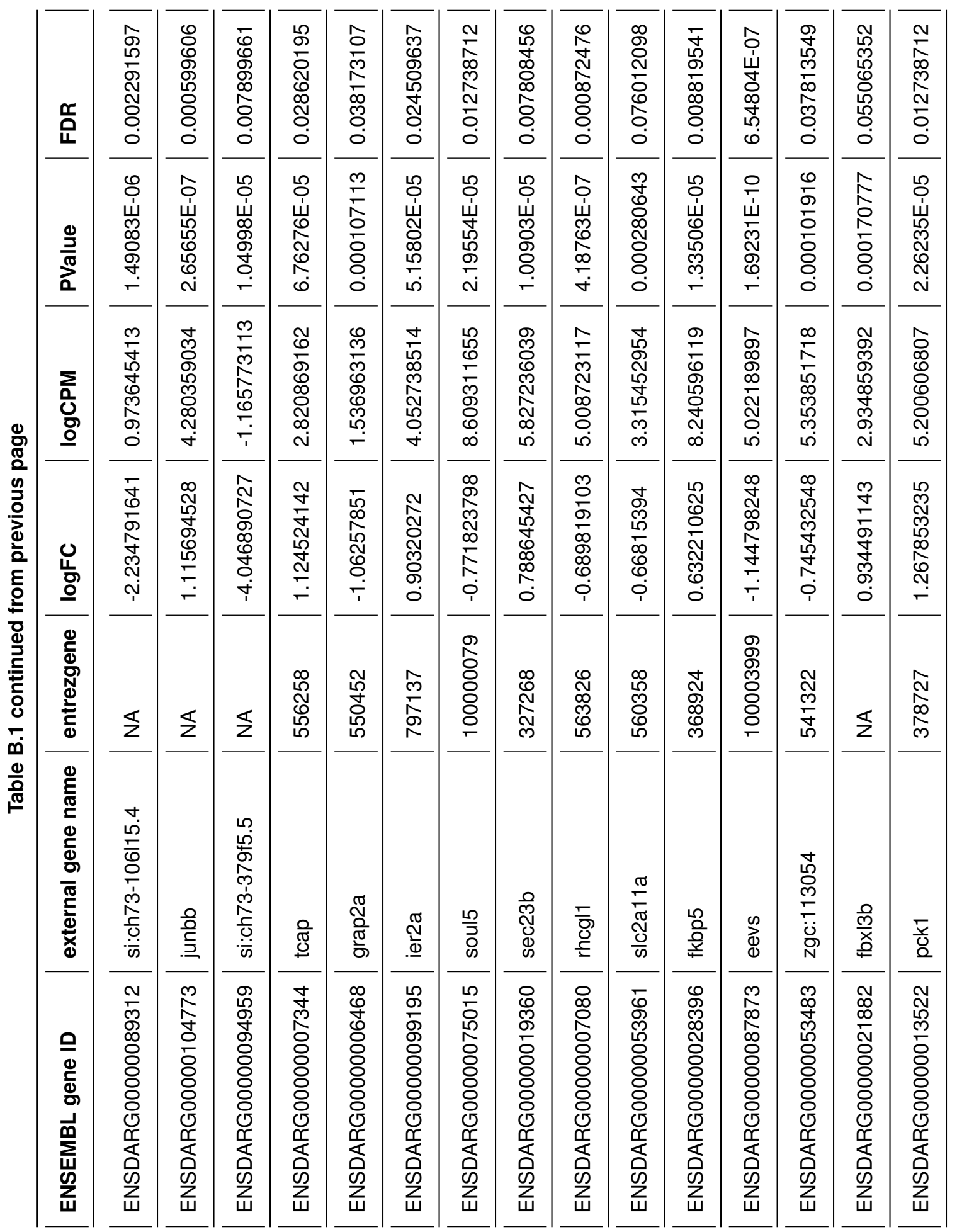




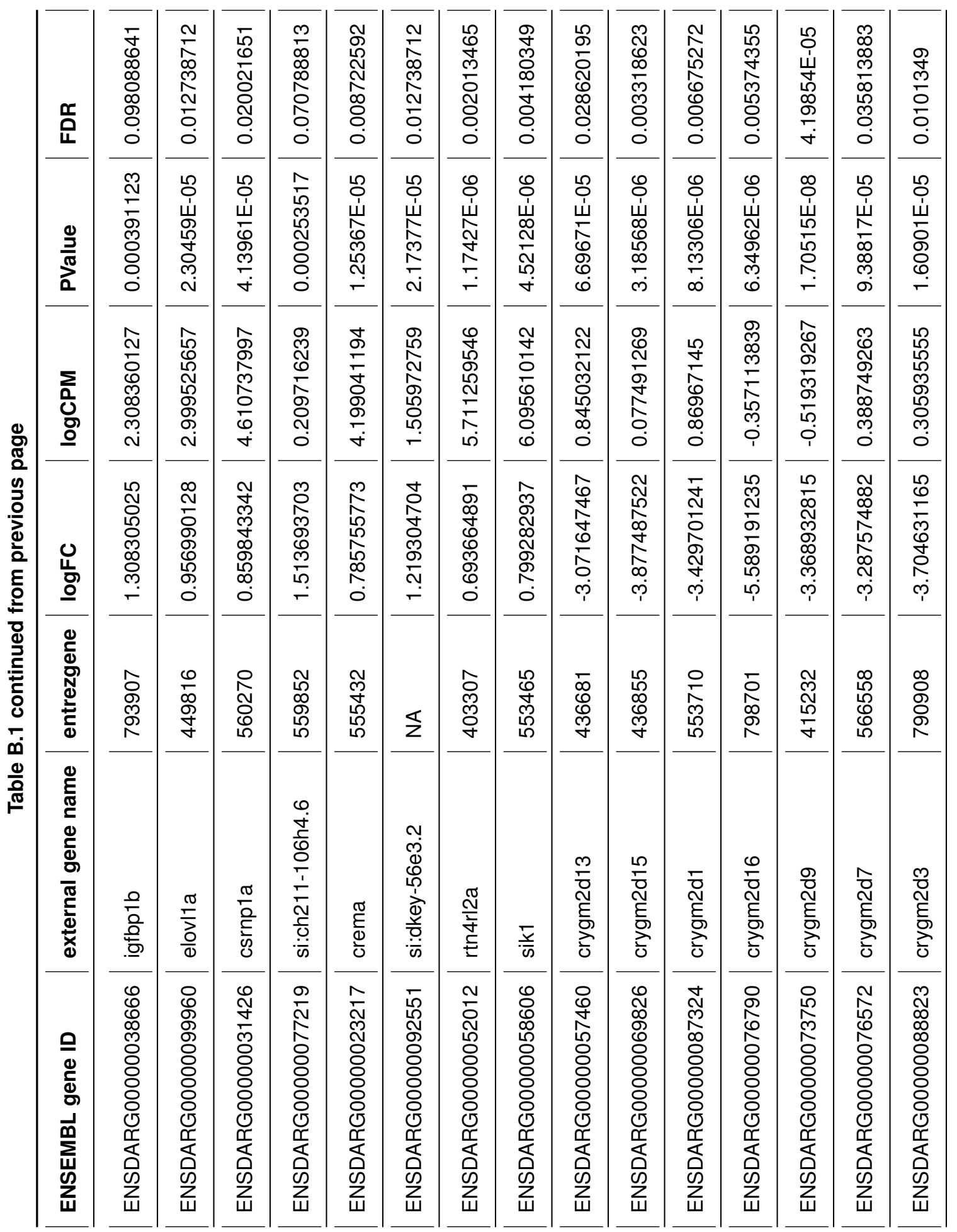




\begin{tabular}{|c|c|c|c|c|c|c|c|c|c|c|c|c|c|c|c|}
\hline $\begin{array}{l}\text { 号 } \\
\text { L }\end{array}$ & 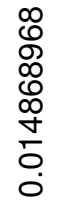 & 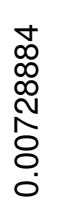 & 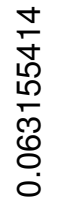 & 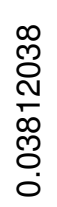 & 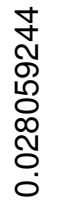 & 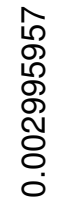 & $\begin{array}{l}\text { ? } \\
\text { లో } \\
\infty \\
0 \\
0 \\
\infty \\
0 \\
0 \\
0\end{array}$ & 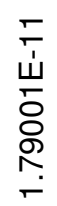 & $\begin{array}{l}\infty \\
\stackrel{N}{N} \\
\stackrel{0}{0} \\
\text { No } \\
0 \\
0\end{array}$ & 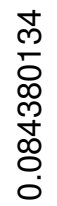 & 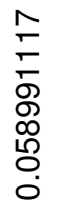 & $\begin{array}{l}\frac{\infty}{0} \\
\infty \\
\bar{\delta} \\
\bar{\sigma} \\
\vdots \\
0\end{array}$ & 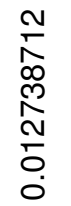 & 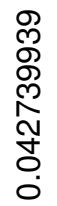 & 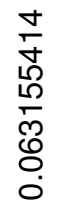 \\
\hline$\frac{\stackrel{0}{\frac{V}{n}}}{\frac{\pi}{\Delta}}$ & 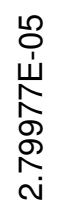 & 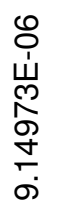 & 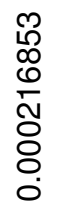 & 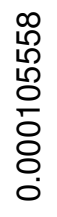 & 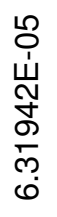 & 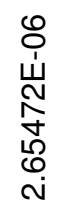 & 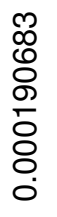 & 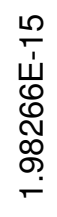 & 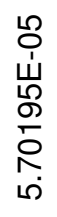 & 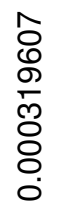 & 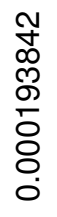 & 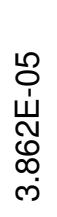 & 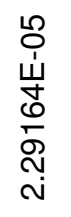 & 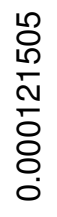 & 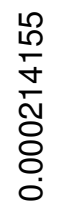 \\
\hline $\begin{array}{l}\Sigma \\
0 \\
0 \\
\text { 으 }\end{array}$ & 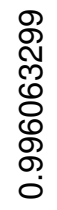 & 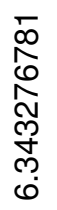 & 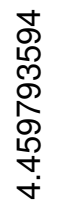 & 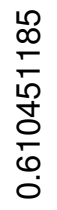 & 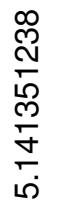 & 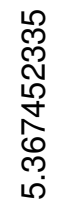 & 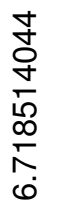 & 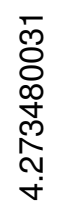 & 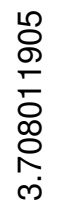 & 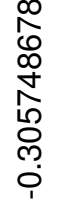 & \begin{tabular}{l} 
N \\
$\infty$ \\
$o$ \\
\multirow{O}{0}{} \\
$\stackrel{N}{N}$ \\
in
\end{tabular} & 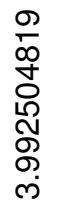 & 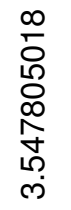 & 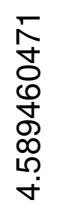 & 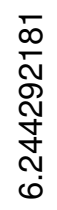 \\
\hline $\begin{array}{l}\text { 는 } \\
\text { 으 }\end{array}$ & 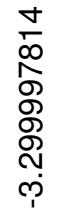 & 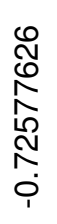 & $\begin{array}{l}\text { के } \\
\text { న } \\
0 \\
0 \\
\mathbb{N} \\
\infty \\
0 \\
0 \\
0\end{array}$ & 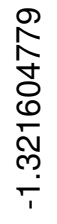 & 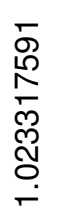 & 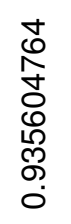 & 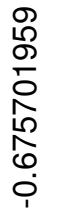 & 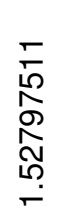 & 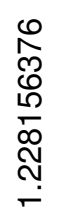 & 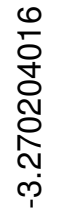 & 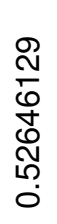 & 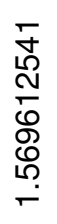 & 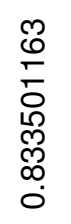 & 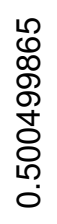 & 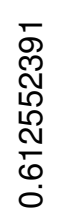 \\
\hline 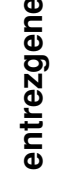 & 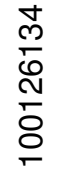 & $\begin{array}{l}\text { Nิ } \\
\text { م్ } \\
\text { o } \\
\text { N }\end{array}$ & $\begin{array}{l}\text { ठ } \\
80 \\
\infty \\
\text { பे }\end{array}$ & $\begin{array}{l}\text { ڤొ } \\
\text { న్ } \\
\text { ని } \\
\text { N }\end{array}$ & $\begin{array}{l}\prod_{0} \\
\frac{0}{\Gamma} \\
\frac{m}{n}\end{array}$ & 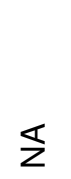 & 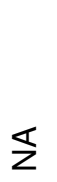 & 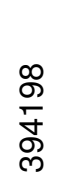 & 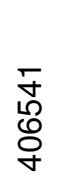 & $\S$ & $\begin{array}{l}0 \\
1 \\
\swarrow 0 \\
0 \\
10\end{array}$ & 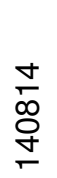 & $\begin{array}{l}\hat{L} \\
\stackrel{0}{0} \\
\stackrel{0}{8}\end{array}$ & 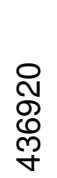 & $\begin{array}{l}\text { 尺े } \\
\text { D্ల్ } \\
\text { స్ల }\end{array}$ \\
\hline 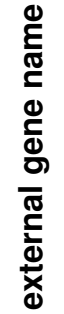 & 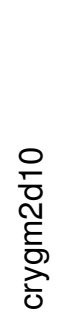 & $\begin{array}{l}\frac{10}{0} \\
\frac{0}{\pi}\end{array}$ & 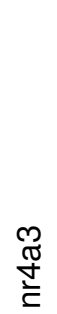 & $\frac{\frac{10}{\infty}}{\frac{1}{1}}$ & $\begin{array}{l}\frac{\sigma}{0} \\
\frac{0}{0} \\
\frac{0}{0}\end{array}$ & $\begin{array}{l}\dot{0} \\
\text { D } \\
\stackrel{0}{W} \\
\infty \\
0 \\
\text { Oे }\end{array}$ & 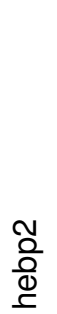 & 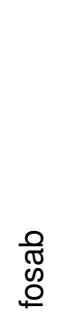 & $\begin{array}{l}\mathbb{0} \\
\stackrel{N}{\mathbb{N}} \\
\widetilde{N} \\
\stackrel{N}{N} \\
\frac{0}{\infty}\end{array}$ & 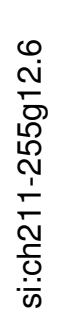 & $\begin{array}{l}\text { ㅇ } \\
\frac{\text { In}}{0}\end{array}$ & $\begin{array}{l}\text { 응 } \\
\frac{0}{0}\end{array}$ & 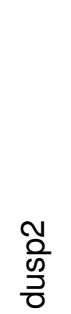 & 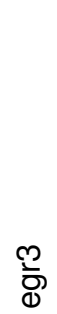 & $\begin{array}{l}\frac{0}{.0} \\
\frac{\pi}{0} \\
\frac{1}{c}\end{array}$ \\
\hline 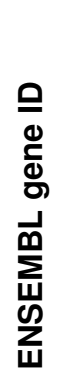 & 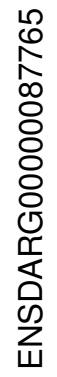 & 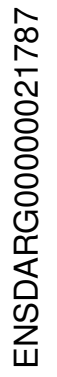 & 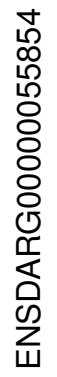 & 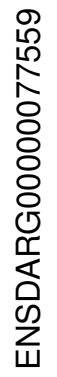 & 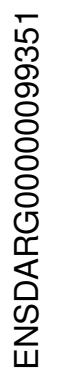 & 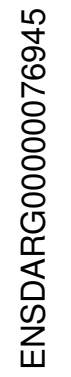 & 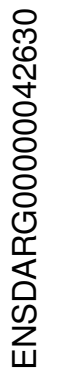 & 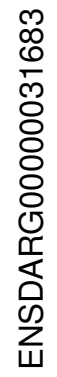 & 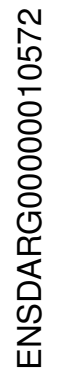 & 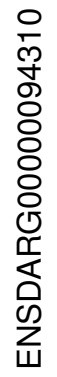 & 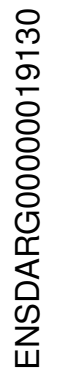 & 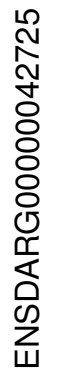 & 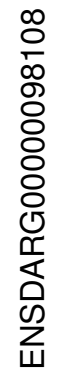 & 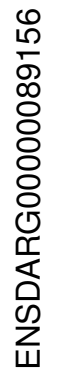 & 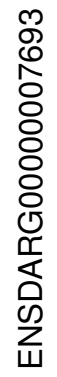 \\
\hline
\end{tabular}




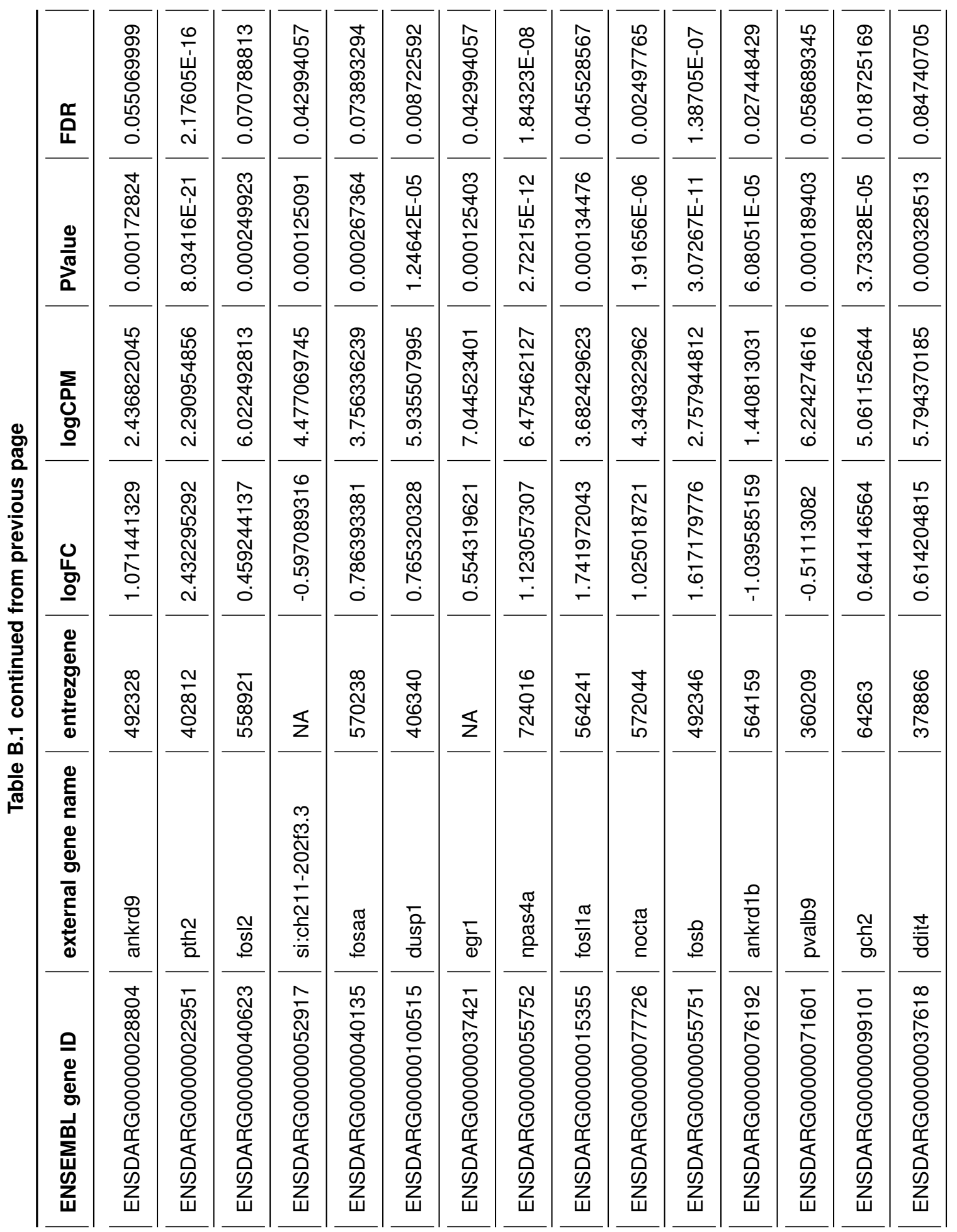




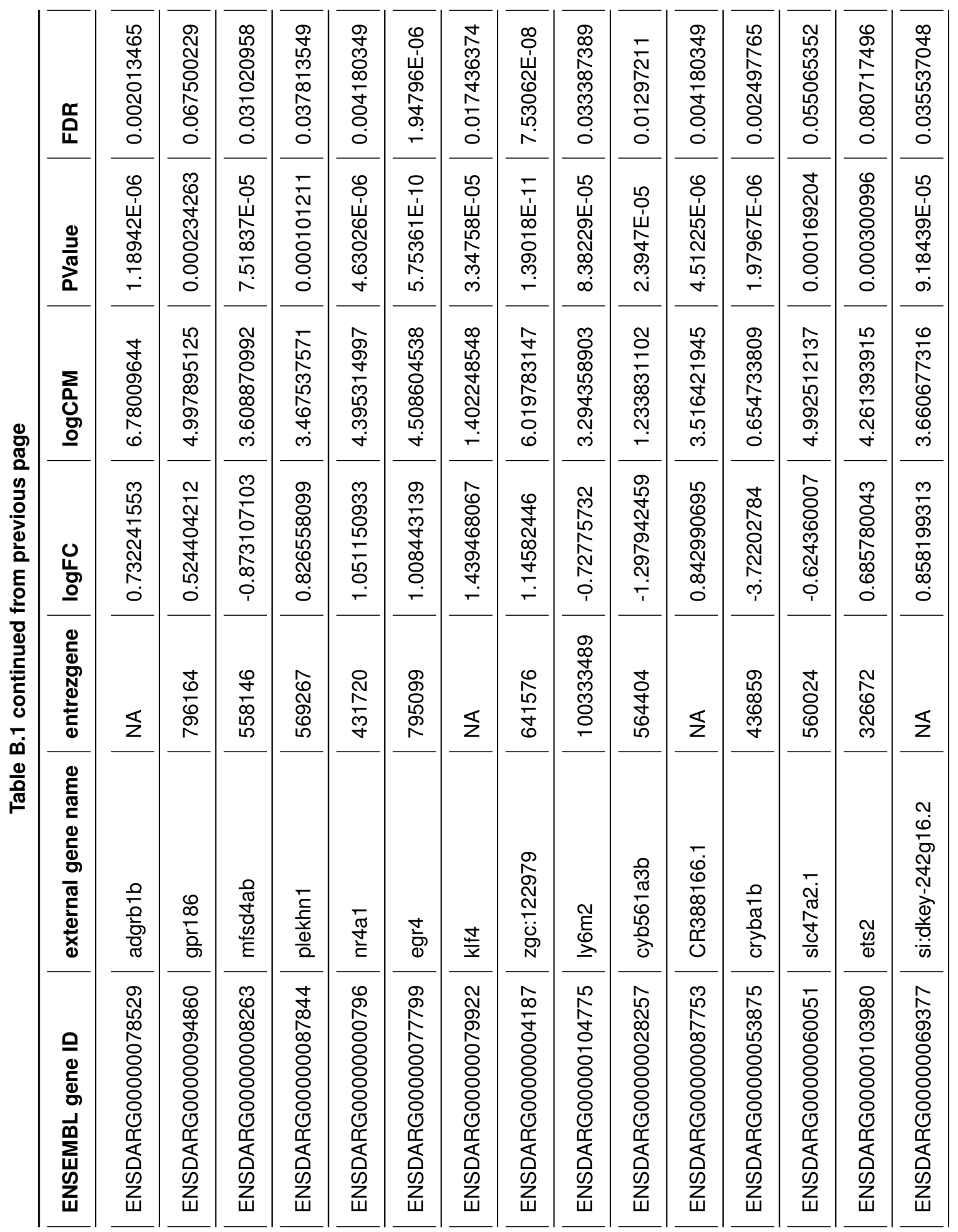




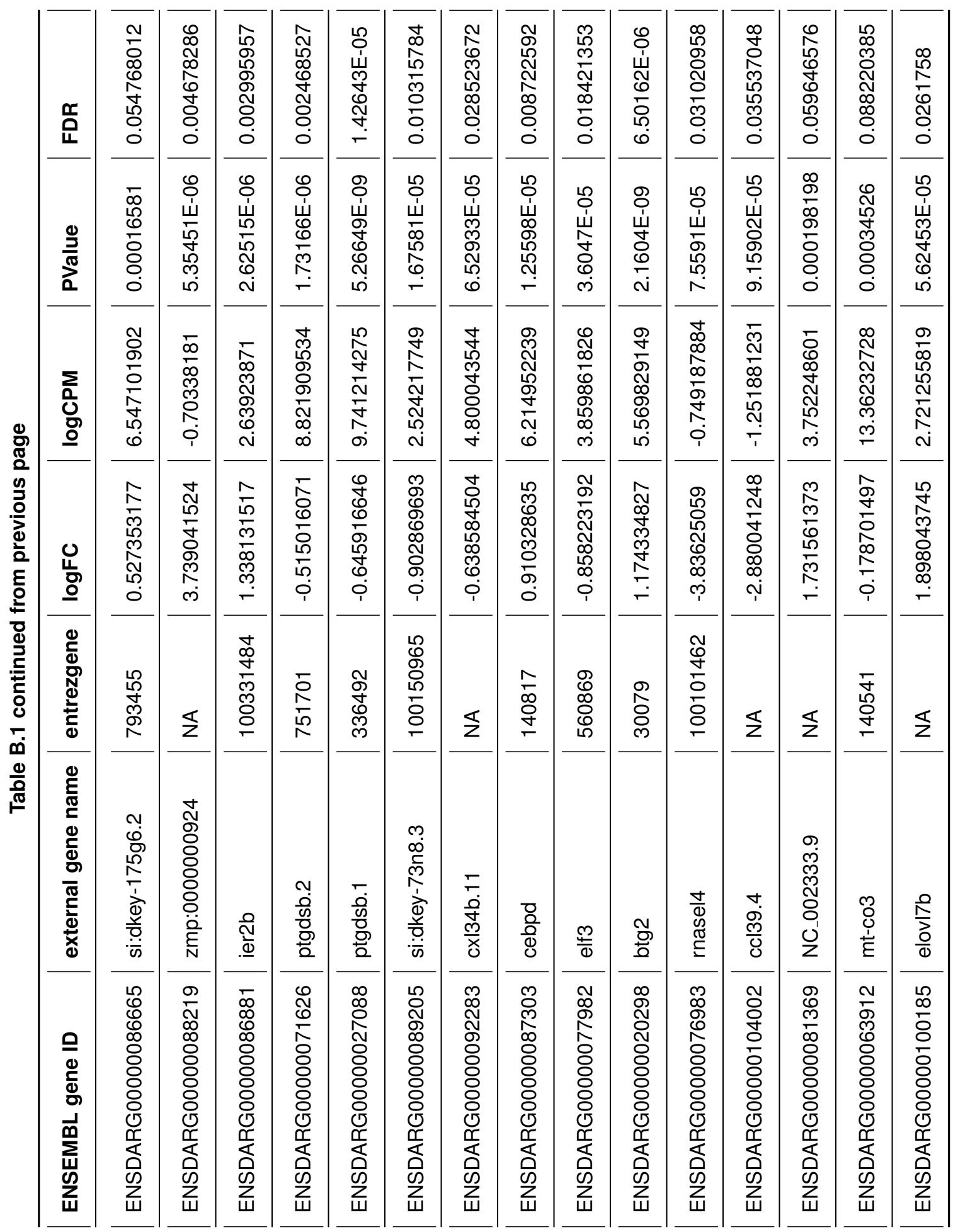




\section{B.2 Differentially expressed genes at $8 \mathrm{dpf}$}

The following table displays all genes that were identified to be differentially expressed in our NGS experiment at $8 \mathrm{dpf}$. 


\begin{tabular}{|c|c|c|c|c|c|c|c|c|c|c|c|c|c|c|c|c|}
\hline 愛 & $\begin{array}{l}0 \\
\infty \\
o \\
\text { o } \\
0 \\
0 \\
0 \\
0\end{array}$ & 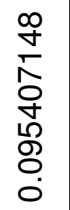 & 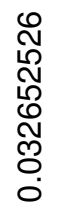 & 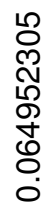 & 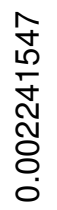 & $\begin{array}{l}\overline{0} \\
\infty \\
0 \\
\\
0 \\
0 \\
0 \\
0 \\
0\end{array}$ & $\begin{array}{l}\text { 寸 } \\
\stackrel{0}{0} \\
\stackrel{N}{N} \\
\text { N } \\
\stackrel{0}{0} \\
0\end{array}$ & $\begin{array}{l}\wp \\
\infty \\
0 \\
0 \\
\infty \\
\infty \\
0 \\
0 \\
0 \\
0\end{array}$ & $\begin{array}{l}\frac{1}{10} \\
\frac{0}{0} \\
\frac{1}{\infty} \\
0 \\
0 \\
0 \\
0\end{array}$ & \begin{tabular}{l}
\multirow{J}{*}{} \\
N \\
$\stackrel{8}{0}$ \\
0 \\
0 \\
0 \\
0
\end{tabular} & 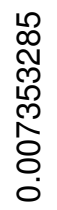 & $\begin{array}{l}\text { م } \\
\hat{N} \\
0 \\
\hat{2} \\
\hat{2} \\
0 \\
0 \\
0\end{array}$ & 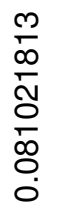 & $\begin{array}{l}N \\
N \\
\hat{N} \\
N \\
0 \\
0 \\
0\end{array}$ & 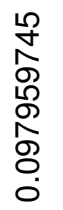 & 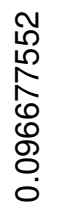 \\
\hline$\frac{\text { D }}{\frac{D}{\pi}}$ & $\begin{array}{l}0 \\
\frac{0}{0} \\
\frac{1}{10} \\
\overline{8} \\
8 \\
0\end{array}$ & $\begin{array}{l}0 \\
8 \\
8 \\
80 \\
0 \\
8 \\
\circ \\
0\end{array}$ & 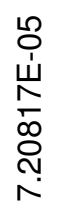 & 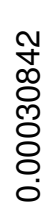 & 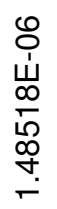 & $\begin{array}{l}\bar{\delta} \\
\text { o } \\
\frac{1}{0} \\
\overline{0} \\
8 \\
0 \\
0\end{array}$ & 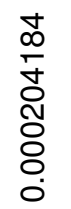 & 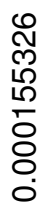 & \begin{tabular}{l}
$\hat{\infty}$ \\
\multirow{0}{0}{} \\
$\stackrel{N}{N}$ \\
$\hat{ᄋ}$ \\
$\stackrel{0}{0}$ \\
0
\end{tabular} & 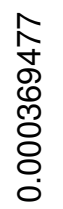 & 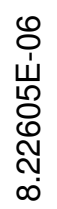 & 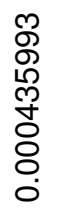 & 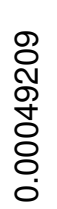 & 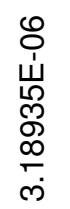 & 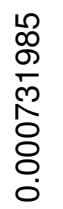 & 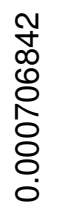 \\
\hline $\begin{array}{l}\Sigma \\
\text { D } \\
\text { o } \\
\text { 으 }\end{array}$ & 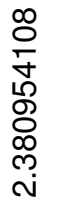 & 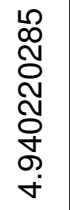 & $\begin{array}{l}\hat{O} \\
\dot{J} \\
\stackrel{N}{N} \\
\stackrel{N}{N} \\
\stackrel{N}{n} \\
\omega\end{array}$ & 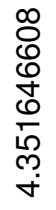 & 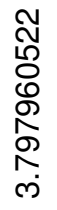 & 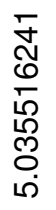 & 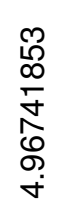 & 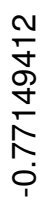 & 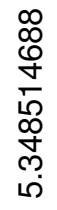 & 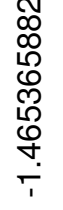 & 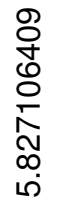 & 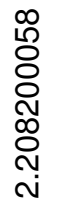 & 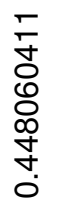 & 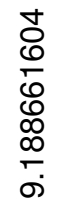 & $\begin{array}{l}0 \\
\stackrel{N}{ } \\
0 \\
\emptyset \\
\stackrel{0}{0} \\
\stackrel{N}{N} \\
\stackrel{+}{+}\end{array}$ & 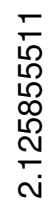 \\
\hline $\begin{array}{l}\text { 늠 } \\
\text { 으 }\end{array}$ & 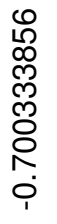 & 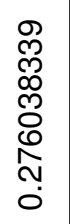 & 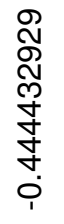 & 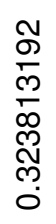 & 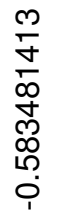 & 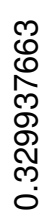 & 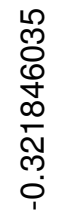 & 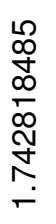 & 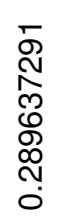 & 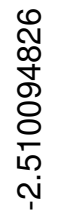 & 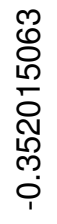 & $\begin{array}{l}\mathscr{W} \\
0 \\
\infty \\
0 \\
0 \\
0 \\
0 \\
0 \\
0 \\
i\end{array}$ & 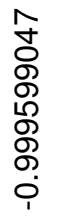 & 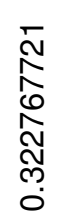 & 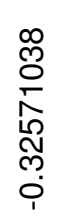 & 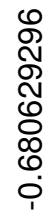 \\
\hline 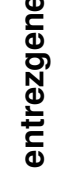 & 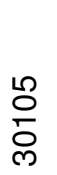 & 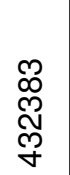 & $\begin{array}{l}\text { O్రి } \\
\text { ర্ণ }\end{array}$ & 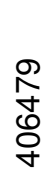 & 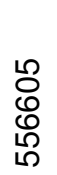 & $\Sigma$ & 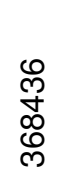 & 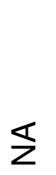 & 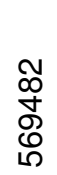 & $\Sigma$ & 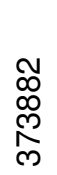 & 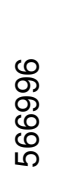 & 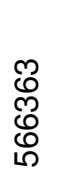 & 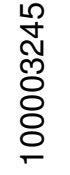 & $\begin{array}{l}\text { o } \\
\text { D } \\
\text { D } \\
\hat{m}\end{array}$ & 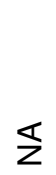 \\
\hline 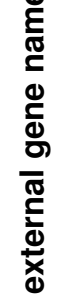 & 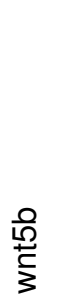 & 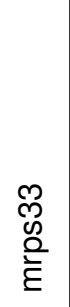 & 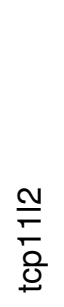 & $\begin{array}{l}\frac{1}{0} \\
0\end{array}$ & $\begin{array}{l}\text { 음 } \\
\text { 을 } \\
\text { 음 }\end{array}$ & $\frac{N}{\frac{N}{\sigma}}$ & $\frac{\frac{\pi}{1}}{\frac{0}{D}}$ & 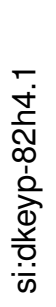 & $\begin{array}{l}\frac{N}{N} \\
\frac{\pi}{0} \\
\frac{0}{0}\end{array}$ & 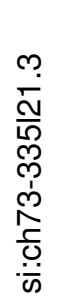 & $\frac{\stackrel{\sigma}{+}}{\frac{\sigma}{E}}$ & 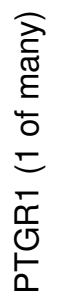 & 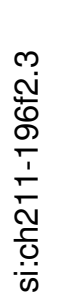 & 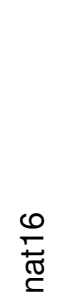 & \begin{tabular}{l} 
D \\
N \\
\multirow{N}{*}{} \\
$\stackrel{D}{N}$
\end{tabular} & $\begin{array}{l}\text { 옹 } \\
\text { 음 } \\
\text { б }\end{array}$ \\
\hline 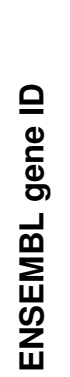 & 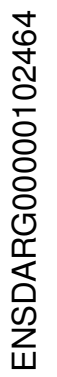 & 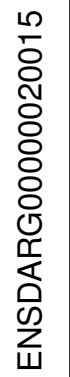 & 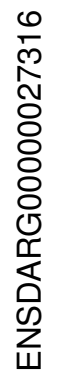 & 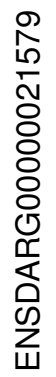 & 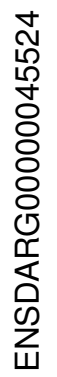 & 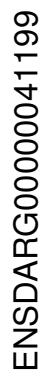 & 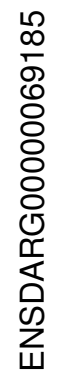 & 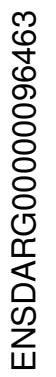 & 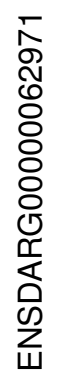 & 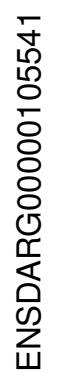 & 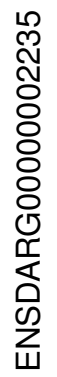 & 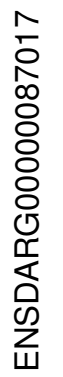 & 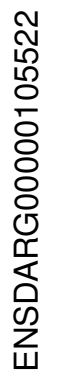 & 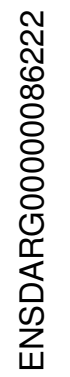 & 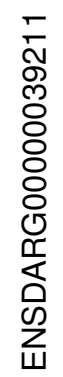 & 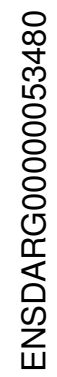 \\
\hline
\end{tabular}




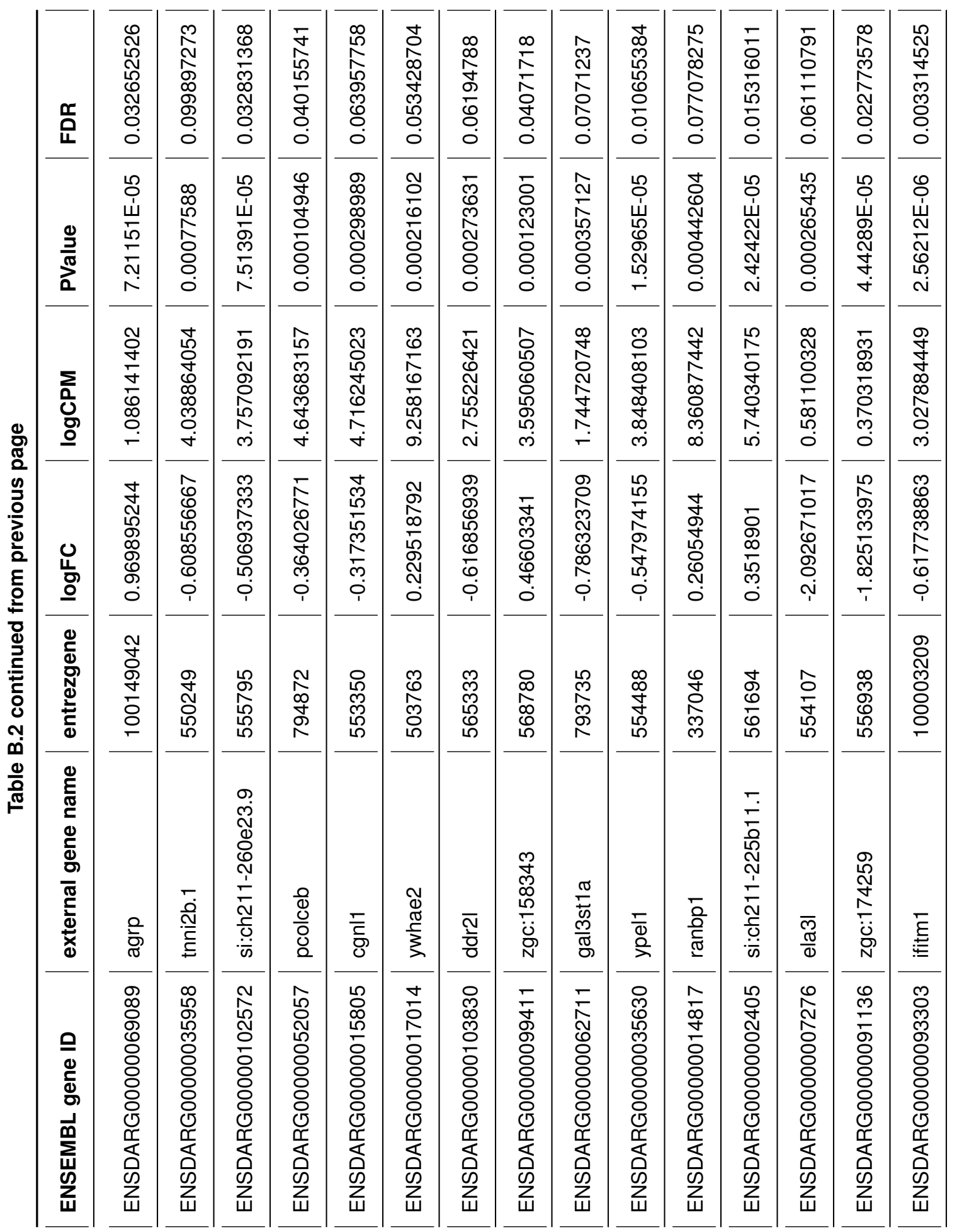




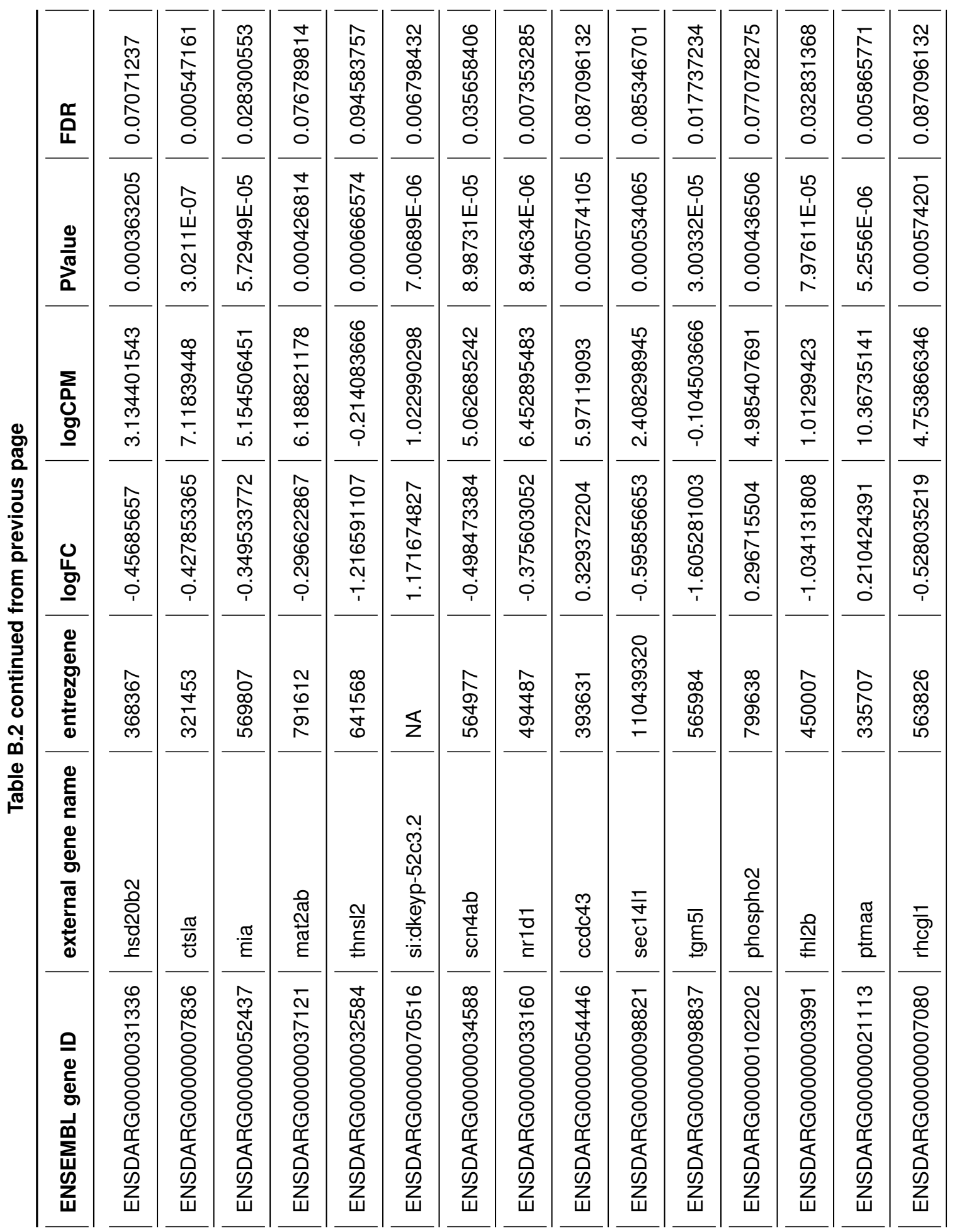




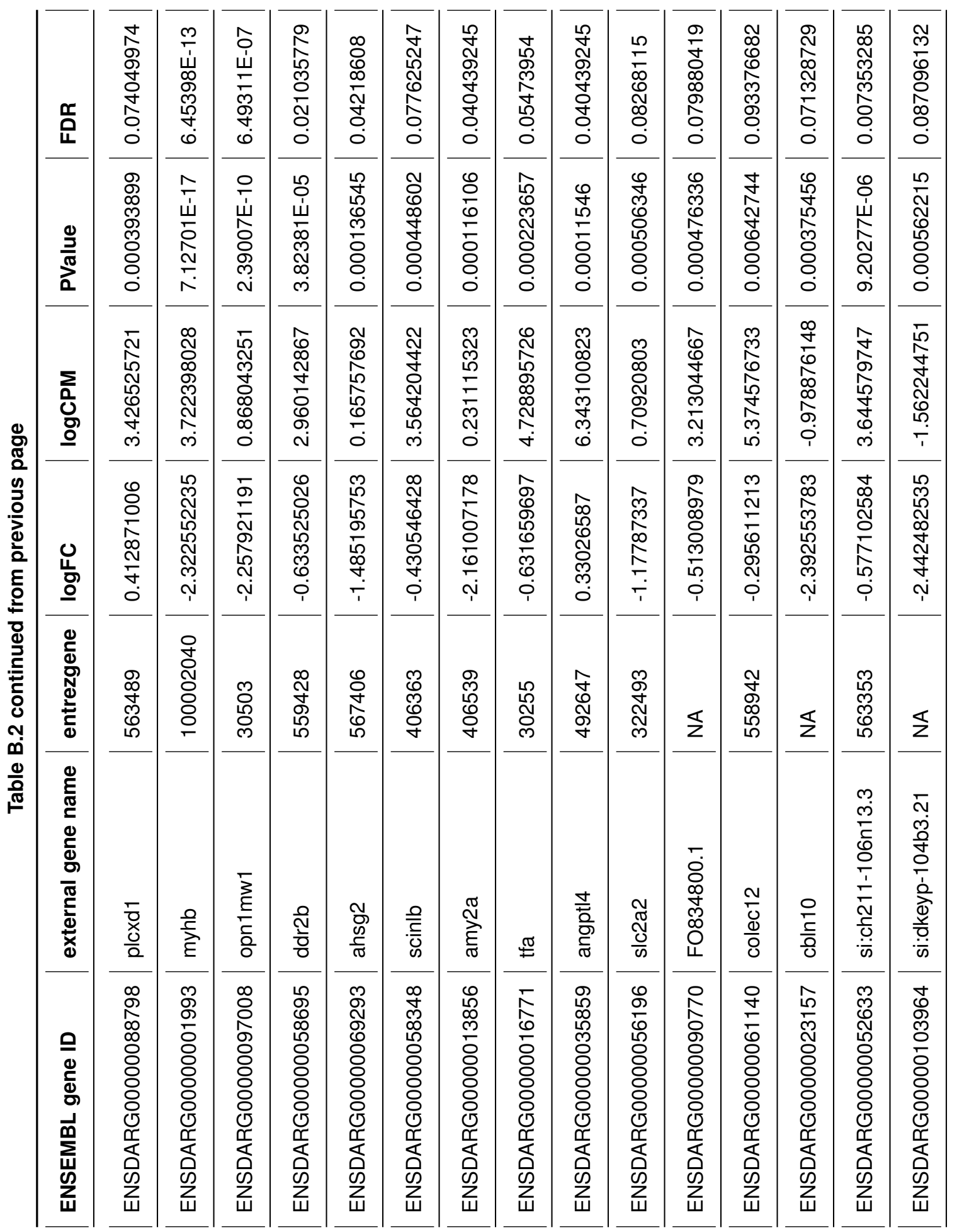




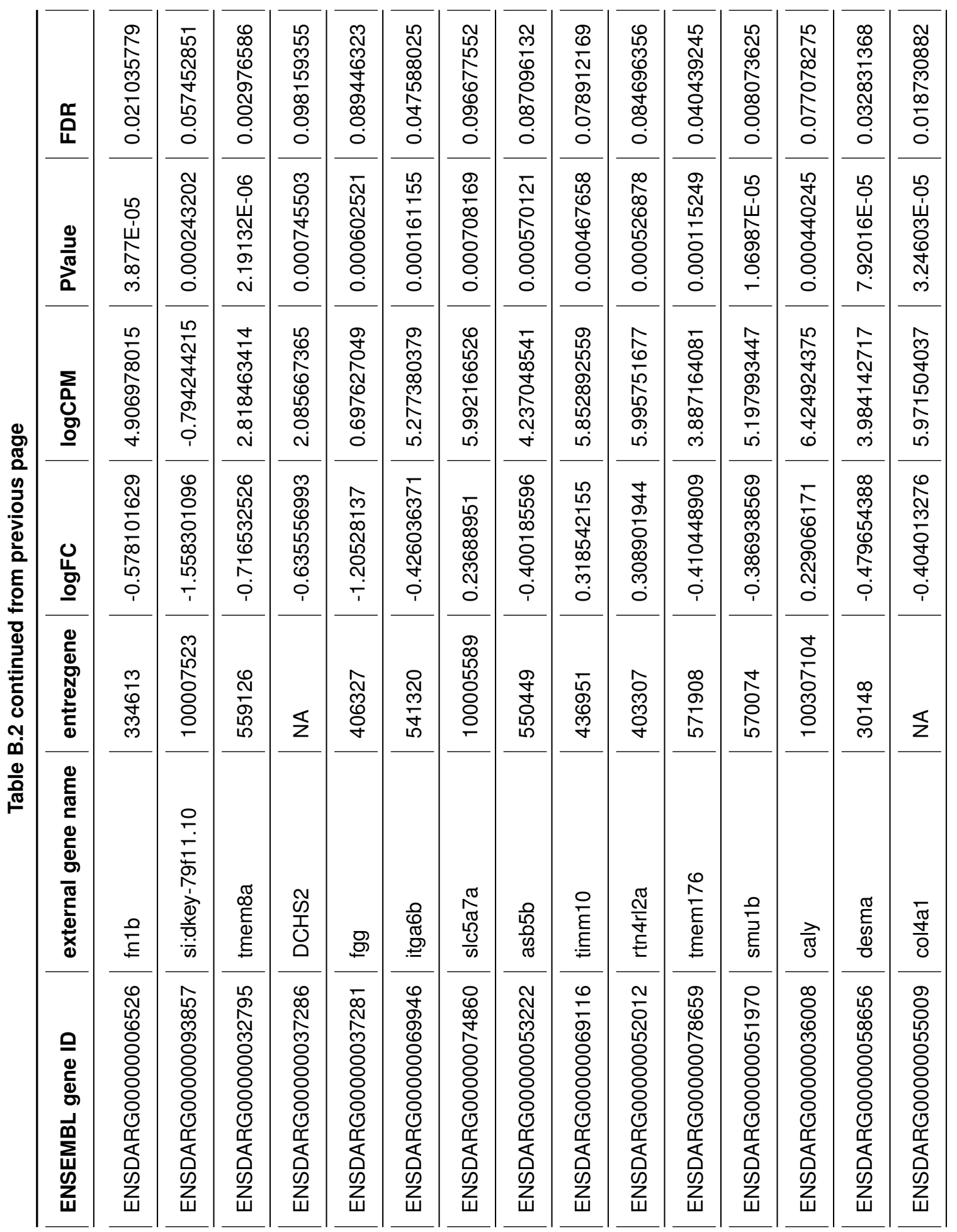




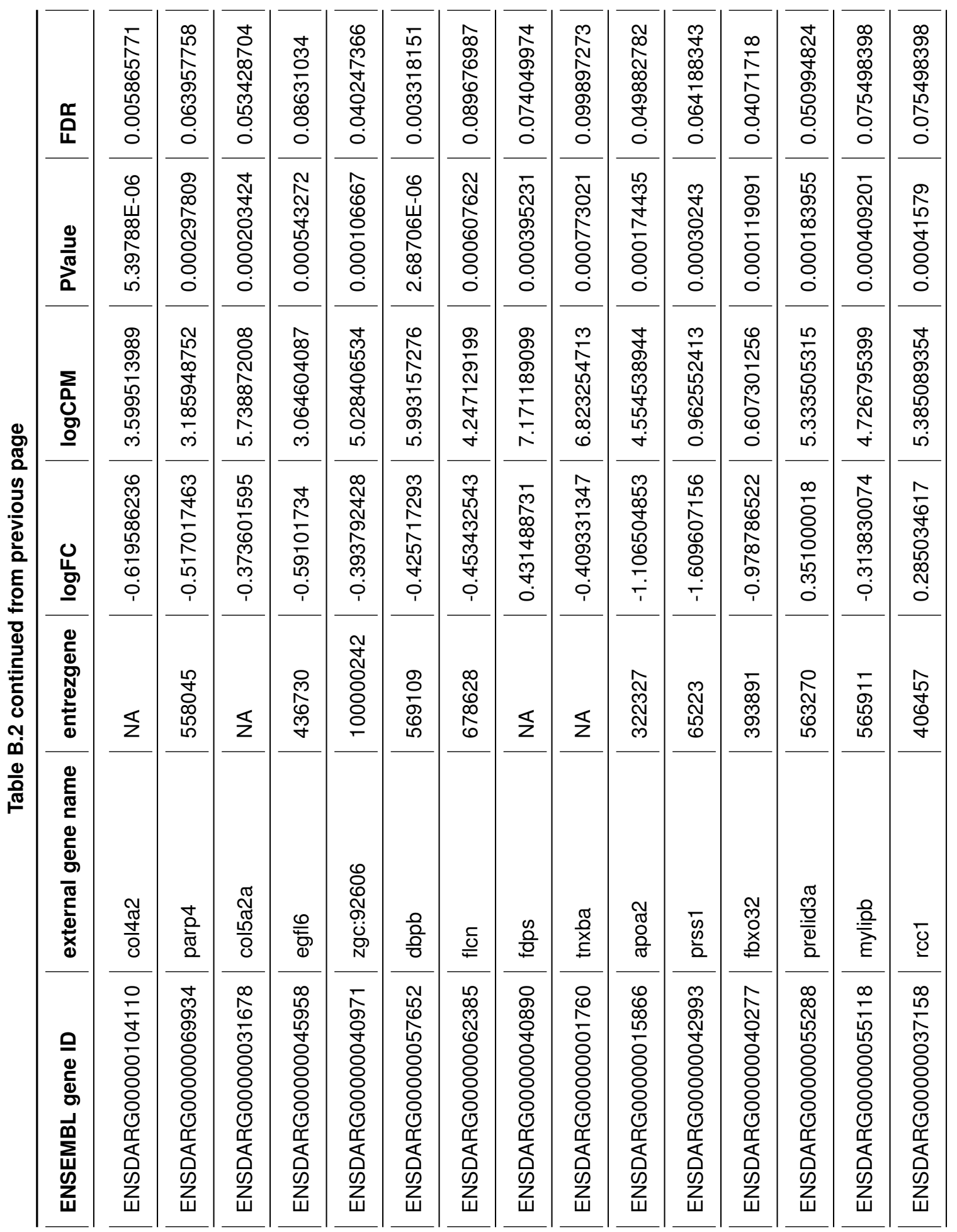




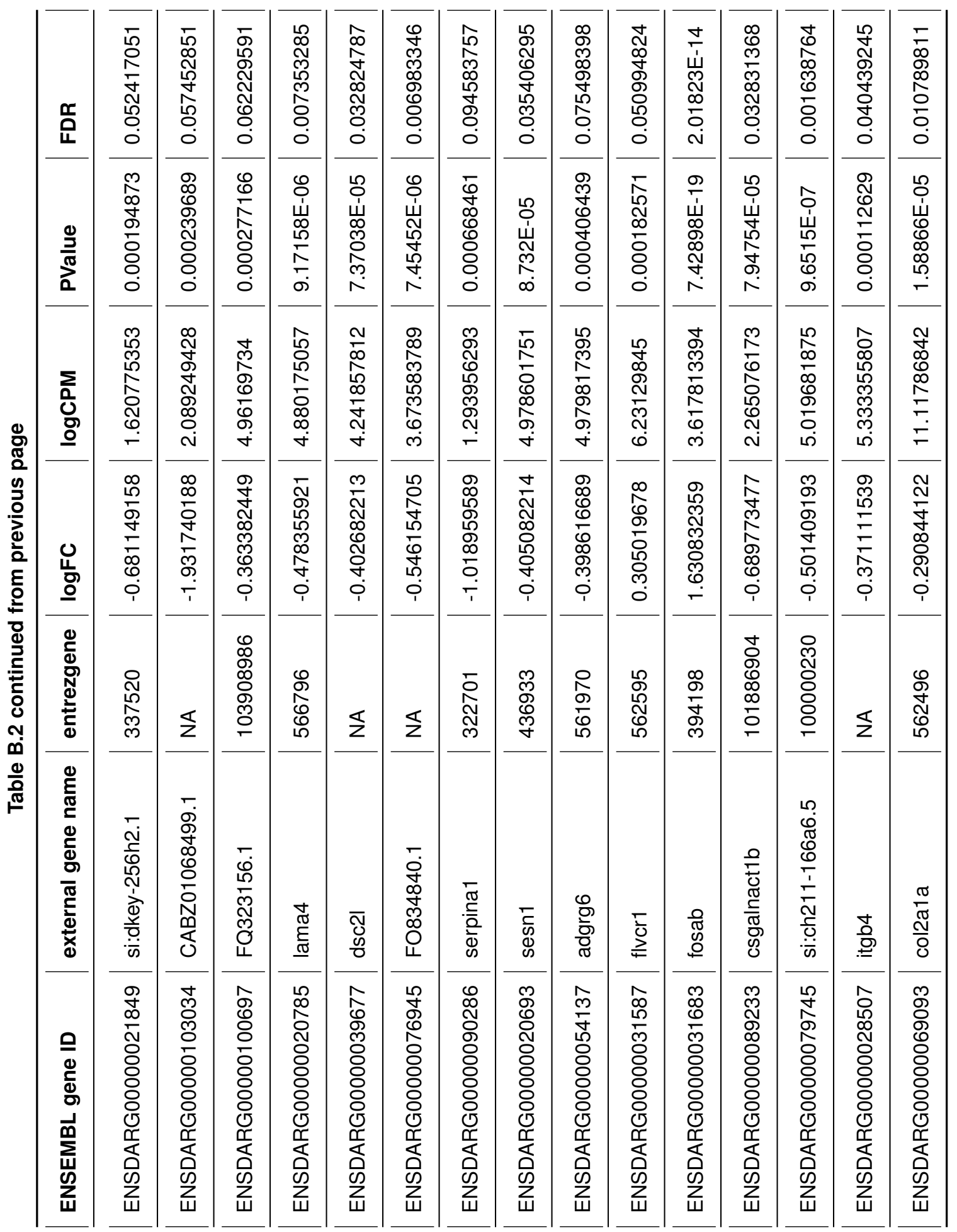




\section{B.3 Differentially expressed genes at $14 \mathrm{dpf}$}

The following table displays all genes that were identified to be differentially expressed in our NGS experiment at $14 \mathrm{dpf}$. 


\begin{tabular}{|c|c|c|c|c|c|c|c|c|c|c|c|}
\hline 愛 & 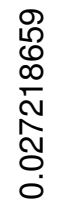 & $\begin{array}{l}\text { N } \\
\text { N } \\
N \\
\hat{N} \\
\text { లె } \\
0 \\
0\end{array}$ & $\begin{array}{l}\text { م } \\
\mathbb{0} \\
\mathbb{\infty} \\
\text { N } \\
N \\
0 \\
0\end{array}$ & 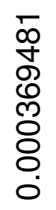 & 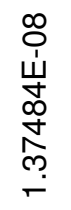 & 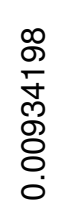 & $\begin{array}{l}N \\
N \\
N \\
N \\
\hat{N} \\
\text { N } \\
0 \\
0\end{array}$ & 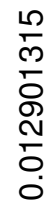 & 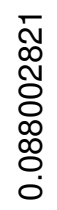 & 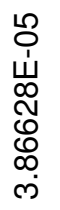 & 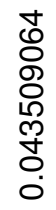 \\
\hline$\frac{0}{\frac{0}{\pi}}$ & 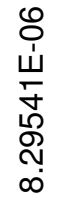 & 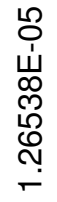 & 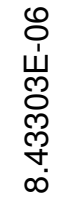 & 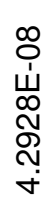 & 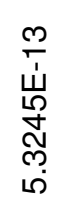 & 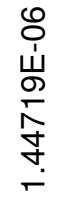 & 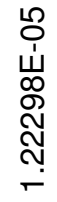 & 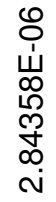 & 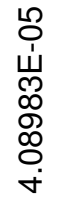 & 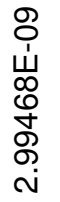 & 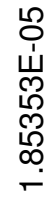 \\
\hline $\begin{array}{l}\Sigma \\
\mathbf{0} \\
0 \\
\text { o }\end{array}$ & 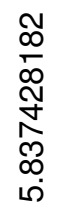 & 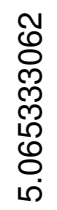 & $\begin{array}{l}\text { مి } \\
\text { N } \\
\infty \\
\& \\
\infty \\
\infty \\
\text { N }\end{array}$ & 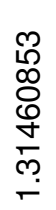 & 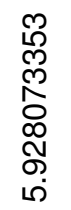 & 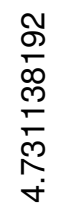 & 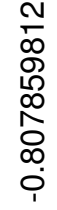 & 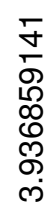 & 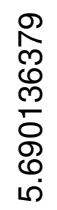 & 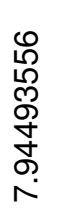 & 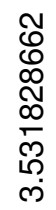 \\
\hline $\begin{array}{l}0 \\
\underline{4} \\
\underline{0}\end{array}$ & $\begin{array}{l}\hat{N} \\
N \\
N \\
0 \\
0 \\
0 \\
م \\
0 \\
0\end{array}$ & 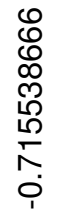 & 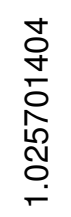 & 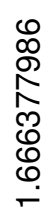 & 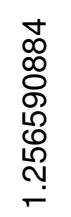 & 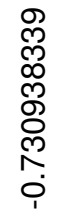 & $\begin{array}{l}\text { N } \\
\text { N } \\
\text { No } \\
\stackrel{0}{0} \\
\stackrel{0}{0} \\
\text { N }\end{array}$ & 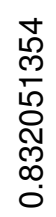 & \begin{tabular}{l} 
N \\
$\stackrel{N}{N}$ \\
o \\
\multirow{2}{*}{} \\
0
\end{tabular} & 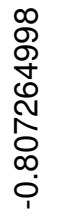 & 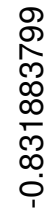 \\
\hline 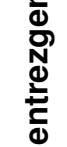 & $\begin{array}{l}\text { గ్ల } \\
\text { ల్ } \\
\text { م⿱ }\end{array}$ & 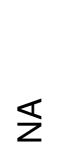 & 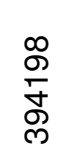 & $\begin{array}{l}\stackrel{N}{\infty} \\
\stackrel{\infty}{N} \\
\text { Oे }\end{array}$ & 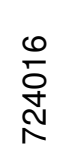 & \begin{tabular}{l}
$\infty$ \\
$\stackrel{\infty}{10}$ \\
$\frac{1}{8}$ \\
\multirow{J}{f}{}
\end{tabular} & 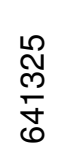 & 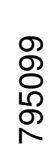 & 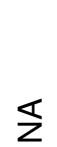 & $\frac{\infty}{\frac{\infty}{\sigma}}$ & 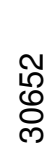 \\
\hline 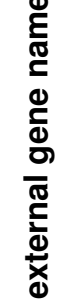 & 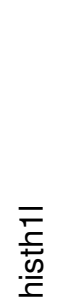 & $\underset{⿱ ㇒}{\stackrel{x}{E}}$ & $\begin{array}{l}\text { ర్లి } \\
\text { రొల్ }\end{array}$ & $\frac{\text { N}}{\stackrel{t}{2}}$ & 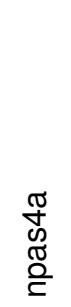 & $\begin{array}{l}\text { Oㅇ } \\
\mathbb{0} \\
\frac{0}{0} \\
\frac{0}{\omega}\end{array}$ & 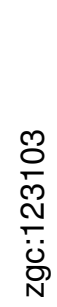 & ఫ্ঠ) & 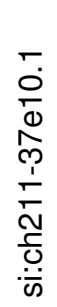 & 올 & 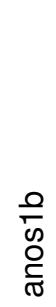 \\
\hline 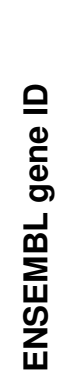 & 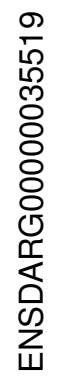 & 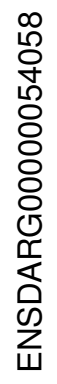 & 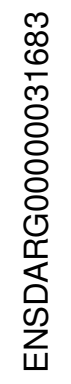 & 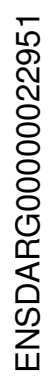 & 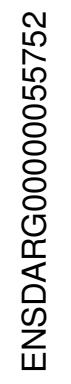 & 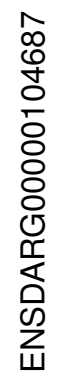 & 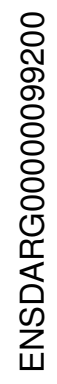 & 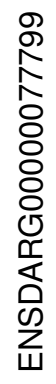 & 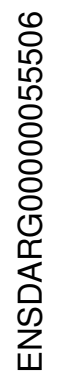 & 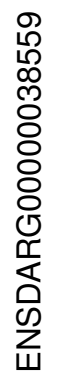 & 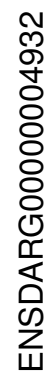 \\
\hline
\end{tabular}




\section{B.4 Differentially expressed genes at $21 \mathrm{dpf}$}

The following table displays all genes that were identified to be differentially expressed in our NGS experiment at $21 \mathrm{dpf}$. 


\begin{tabular}{|c|c|c|c|c|c|c|c|c|c|c|c|c|c|c|c|c|}
\hline $\begin{array}{l}\text { 采 } \\
\text {. }\end{array}$ & 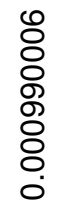 & 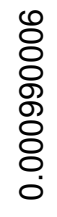 & 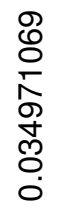 & 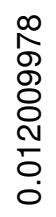 & $\begin{array}{l}\text { N్ } \\
\text { О్ } \\
\text { స్ } \\
\text { ర్ } \\
0\end{array}$ & 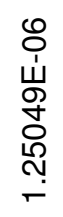 & $\begin{array}{l}\hat{N} \\
\bar{N} \\
0 \\
\stackrel{0}{0} \\
0 \\
0 \\
0\end{array}$ & 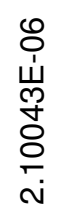 & 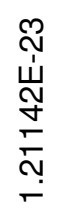 & 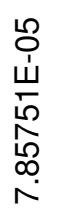 & 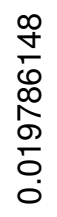 & 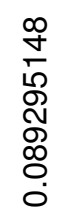 & $\begin{array}{l}N \\
\overline{\dot{u}} \\
\underset{N}{N} \\
\stackrel{N}{0} \\
\hat{N}\end{array}$ & 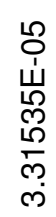 & 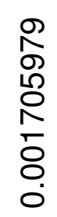 & 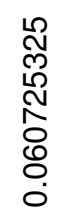 \\
\hline 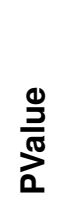 & 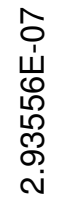 & 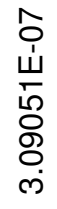 & 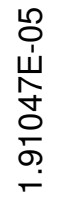 & $\begin{array}{l}0 \\
0 \\
\dot{1} \\
\qquad ో 1 \\
0 \\
0 \\
00 \\
0 \\
\forall\end{array}$ & 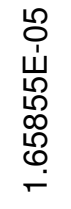 & 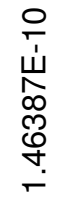 & 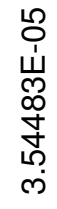 & 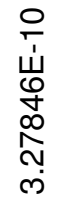 & 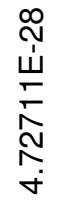 & 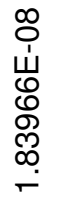 & 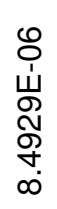 & 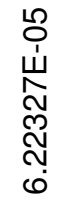 & 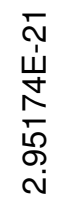 & \begin{tabular}{l} 
o \\
$\dot{1}$ \\
क \\
\multirow{+}{0}{} \\
0 \\
0 \\
$\dot{0}$ \\
0
\end{tabular} & 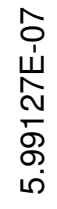 & 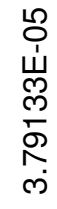 \\
\hline $\begin{array}{l}\sum \\
0 \\
0 \\
\text { O }\end{array}$ & 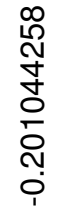 & 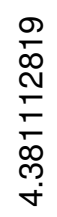 & 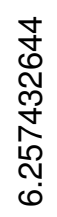 & 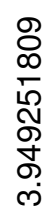 & 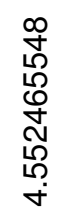 & 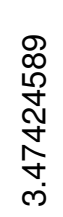 & 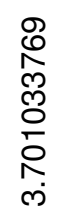 & 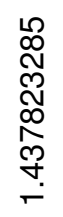 & 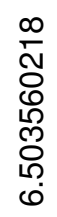 & 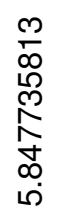 & 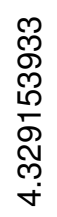 & 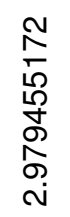 & 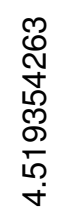 & 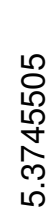 & 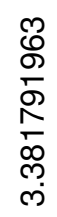 & 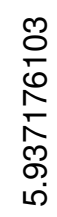 \\
\hline $\begin{array}{l}\text { 늠 } \\
\text { 으 }\end{array}$ & 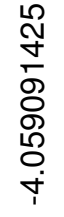 & 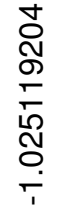 & $\begin{array}{l}\text { N } \\
\text { N} \\
\stackrel{N}{N} \\
\stackrel{N}{N} \\
N \\
N \\
0 \\
0\end{array}$ & $\begin{array}{l}\text { m } \\
0 \\
0 \\
8 \\
0 \\
0 \\
0 \\
\infty \\
0\end{array}$ & $\begin{array}{l}\bar{\sigma} \\
\bar{\sigma} \\
\frac{1}{0} \\
0 \\
0 \\
0\end{array}$ & 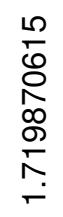 & 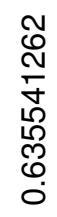 & 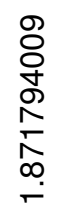 & 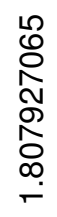 & 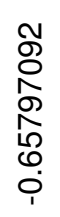 & $\begin{array}{l}0 \\
\infty \\
10 \\
\infty \\
\infty \\
\infty \\
0 \\
0 \\
\infty \\
0\end{array}$ & $\begin{array}{l}\text { N } \\
\text { N } \\
\stackrel{N}{N} \\
\stackrel{N}{N} \\
\stackrel{5}{r}\end{array}$ & 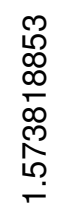 & 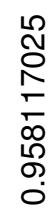 & $\begin{array}{l}\hat{N} \\
\infty \\
\infty \\
\infty \\
0 \\
\infty \\
0 \\
\infty \\
0 \\
0\end{array}$ & 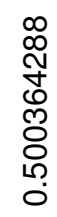 \\
\hline 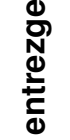 & $\begin{array}{l}8 \\
\stackrel{6}{0} \\
\stackrel{2}{\sigma}\end{array}$ & $\frac{N}{\frac{N}{0}}$ & 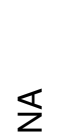 & $\begin{array}{l}\text { مొ } \\
\text { స్లి } \\
\text { లn }\end{array}$ & $\begin{array}{l}\text { N } \\
\text { గ్ } \\
\text { ర్ }\end{array}$ & 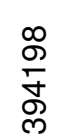 & $\begin{array}{l}\text { : } \\
\text { Oे } \\
\stackrel{+}{N}\end{array}$ & 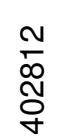 & \begin{tabular}{l}
0 \\
\multirow{0}{+}{} \\
$\stackrel{+}{N}$
\end{tabular} & 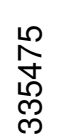 & $\begin{array}{l}\text { U⿺ } \\
\frac{0}{0} \\
\stackrel{0}{N}\end{array}$ & $\frac{\stackrel{N}{N}}{\stackrel{9}{+}}$ & $\begin{array}{l}\text { 尺 } \\
\text { Dे } \\
\text { م) }\end{array}$ & $\begin{array}{l}0 \\
0 \\
\frac{1}{8} \\
\tilde{\sigma}\end{array}$ & 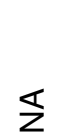 & $\begin{array}{l}\stackrel{L}{\rho} \\
\stackrel{n}{N}\end{array}$ \\
\hline 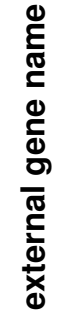 & 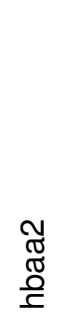 & $\begin{array}{l}\bar{\pi} \\
\frac{0}{0} \\
\frac{0}{\varepsilon}\end{array}$ & 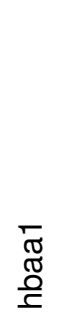 & $\stackrel{\widetilde{\sigma}}{=}$ & 市 & 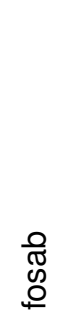 & 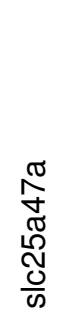 & $\underset{\widetilde{T}}{\tilde{z}}$ & 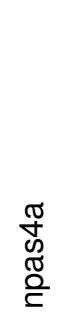 & 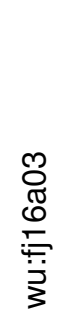 & $\begin{array}{l}\infty \\
\infty \\
\overline{\check{t}} \\
\text { 응 }\end{array}$ & $\stackrel{\bar{\sigma}}{\stackrel{\Xi}{\Xi}}$ & $\stackrel{+}{0}$ & 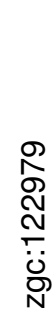 & $\begin{array}{l}\dot{0} \\
\stackrel{0}{0} \\
\infty \\
0 \\
0 \\
\tilde{0} \\
0\end{array}$ & 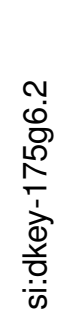 \\
\hline 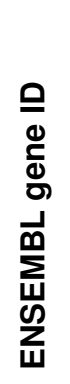 & 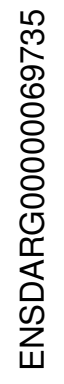 & 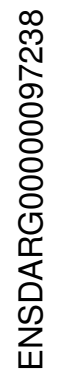 & 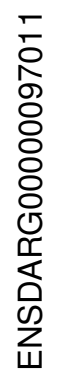 & 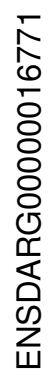 & 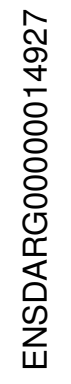 & 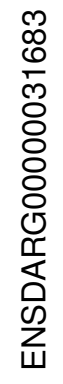 & 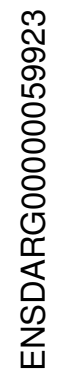 & 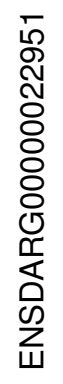 & 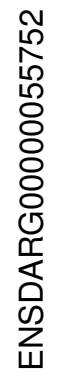 & 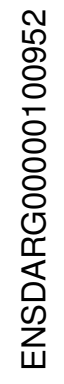 & 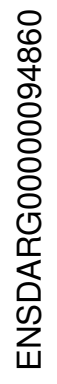 & 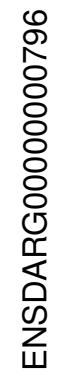 & 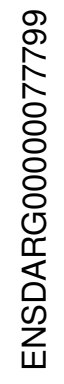 & 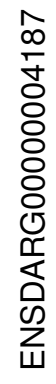 & 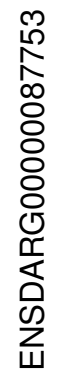 & 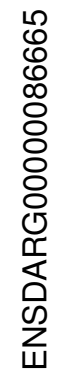 \\
\hline
\end{tabular}




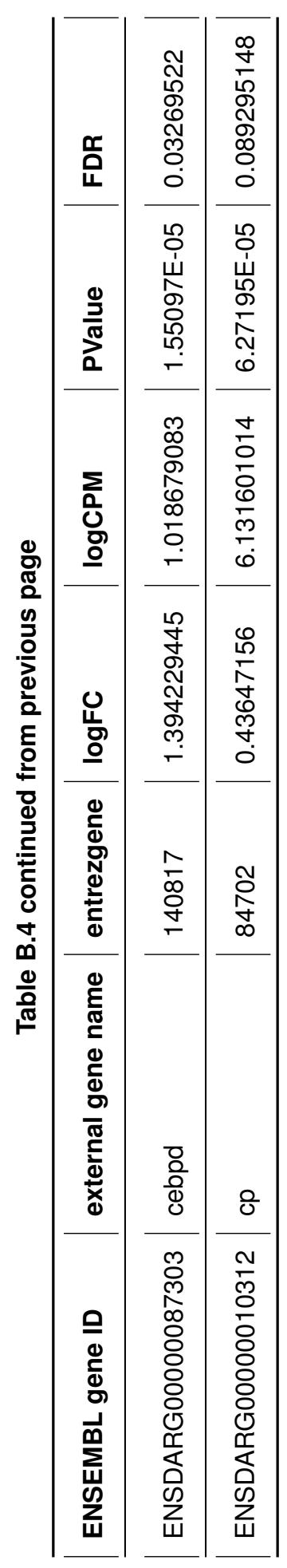


Appendix C

List of Figures 
1.1 Evolution of sociality . . . . . . . . . . . . . . . . . . . . . . . . . 3

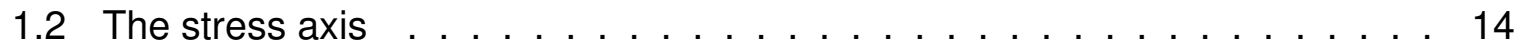

1.3 Development of zebrafish . . . . . . . . . . . . . . . . . . . . . . . . . 20

1.4 The lateral line system . . . . . . . . . . . . . . . . . . . . . . . . . . . . . . 27

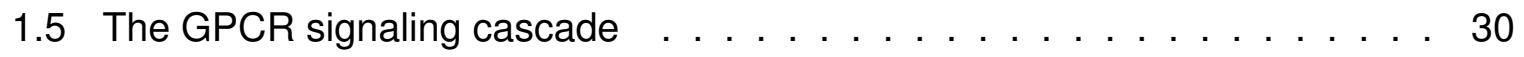

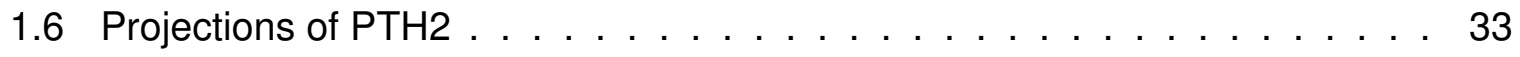

2.1 RNA integrity measurement . . . . . . . . . . . . . . . . . . . . . . . . . . . . . . . . . . . . . 54

2.2 Analysis of social preference . . . . . . . . . . . . . . . . . 53

3.1 Detection of genes . . . . . . . . . . . . . . . . . . . . . 60

3.2 Overlap of differentially expressed genes at different stages . . . . . . . . . 61

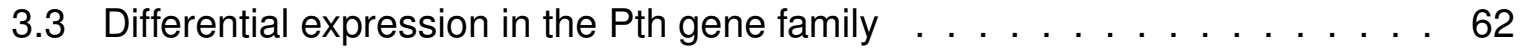

3.4 Expression dynamics of Pth2 . . . . . . . . . . . . . . . . . . . . . . . . . . 64

3.5 Group size dependency of Pth2 expression . . . . . . . . . . . . . . . . . . 65

3.6 Density dependency of Pth2 expression . . . . . . . . . . . . . . . . . . . . 66

3.7 Expression model of Pth2 . . . . . . . . . . . . . . . . . . . . . . 67

3.8 Pth2 expression at different developmental stages . . . . . . . . . . . . . . 69

3.9 Chemosensory access to conspecifics . . . . . . . . . . . . . . . . . . . . 71

3.10 Visual access to conspecifics . . . . . . . . . . . . . . . . . . . . . . . . 73

3.11 Pth2 expression in darkness . . . . . . . . . . . . . . . . . . . . . . . . . 74

3.12 Ablation of the lateral line . . . . . . . . . . . . . . . . . . . . . . 75

3.13 Mechanical social cues are necessary for Pth2 expression . . . . . . . . . . . 77

3.14 Mechanical cues and their impact on Pth2 expression . . . . . . . . . . . . 78

3.15 Artificial stimulation with mechanical cues . . . . . . . . . . . . . . . . . . . 80

3.16 Model of zebrafish movement . . . . . . . . . . . . . . . . . . . . . . . . . . . . . . . . . . . . . . .

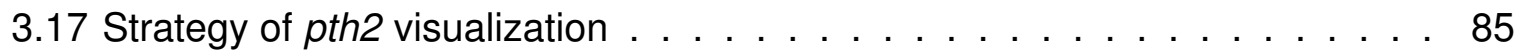

3.18 Visualization of pth2 . . . . . . . . . . . . . . . . . . . . . . . . . . 86

3.19 Registration to zebrafish brain atlas . . . . . . . . . . . . . . . . . . 88

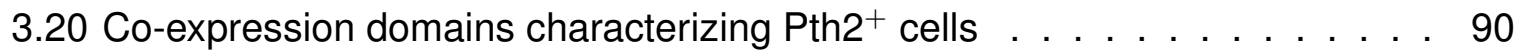

3.21 Expression of $p t h 2 r \ldots \ldots \ldots$. . . . . . . . . . . . . . . . . . . . . 92

3.22 Expression of pth2r in mammals . . . . . . . . . . . . . . . . . . . . . . . 94

3.23 Impact of social isolation on the stress response . . . . . . . . . . . . . . . 96

3.24 Locomotion of mutant fish . . . . . . . . . . . . . . . . . . . . . . . . . . . . . . . . . . . . . . . . . . . . . . . . .

3.25 Startle responses of mutant fish . . . . . . . . . . . . . . . . . . 100 
3.26 Social preference of mutant fish . . . . . . . . . . . . . . . . 101

3.27 Social preference in an open-field paradigm . . . . . . . . . . . . . 102

4.1 Proposed minimal circuit $\ldots \ldots \ldots$. . . . . . . . . . 108 


\section{Bibliography}

[1] J. Liedtke and J. Schneider, Social makes smart: rearing conditions affect learning and social behaviour in jumping spiders, Animal Cognition 20 (2017) 1093-1106.

[2] S. M. Farris, Insect societies and the social brain, Current Opinion in Insect Science 15 (2016) 1-8.

[3] T. Okuyama, S. Yokoi, and H. Takeuchi, Molecular basis of social competence in medaka fish, Development, Growth \& Differentiation 59 (2017) 211-218.

[4] C. Faulkes, N. Bennett, M. Bruford, H. O'brien, G. Aguilar, and J. Jarvis, Ecological constraints drive social evolution in the African mole-rats, Proceedings in the Royal Society of London. Series B: Biological Sciences 264 (1997) 1619-1627.

[5] M. McClure and E. Despland, Defensive responses by a social caterpillar are tailored to different predators and change with larval instar, Naturwissenschaften 98 (2011) 425-434.

[6] T. Clutton-Brock and E. Huchard, Social competition and selection in males and females, Philosophical Transactions of the Royal Society B: Biological Sciences $\mathbf{3 6 8}$ (2013).

[7] E. Wilson and B. Hölldobler, Eusociality: Origin and consequences, Proceedings of the National Academy of Sciences of the United States of America 102 (2005) 13367-13371.

[8] S. Dronjak, L. Gavrilović, D. Filipović, and M. Radojčić, Immobilization and cold stress affect sympatho-adrenomedullary system and pituitary-adrenocortical axis of rats exposed to long-term isolation and crowding, Physiology and Behavior $\mathbf{8 1}$ (2004) 409-415.

[9] K. Nonogaki, K. Nozue, and Y. Oka, Social Isolation Affects the Development of Obesity and Type 2 Diabetes in Mice, Endocrinology 148 (2007) 4658-4666.

[10] H. Ruan and C. Wu, Social interaction-mediated lifespan extension of Drosophila $\mathrm{Cu} / \mathrm{Zn}$ superoxide dismutase mutants, Proceedings of the National Academy of Sciences of the United States of America 105 (2008) 7506-7510. 
[11] M. Bens, K. Szafranksi, S. Holtze, A. Sahm, M. Groth, H. Kestler, T. Hildebrandt, and M. Platzer, Naked mole-rat transcriptome signatures of socially suppressed sexual maturation and links of reproduction to aging, BMC Biology 16 (2018).

[12] D. Queller and J. Strassmann, Eusociality, Current Biology 13 (2003) 861-863.

[13] S. Chak, J. Duffy, K. Hultgren, and D. Rubenstein, Evolutionary transitions towards eusociality in snapping shrimps, Nature Ecology and Evolution 1 (2017).

[14] L. Betzig, Eusociality in History, Human Nature 25 (2014) 80-99.

[15] J. Lopes, R. Abril-de Abreu, and R. Oliveira, Brain Transcriptomic Response to Social Eavesdropping in Zebrafish (Danio rerio), PLOS ONE 10 (2015) e0145801.

[16] A. Morozov and W. Ito, Social modulation of fear: Faciliation vs buffering, Genes, Brain and Behavior 18 (2019).

[17] B. Culbert, K. Gilmour, and S. Balshine, Social buffering of stress in a group-living fish, Proceedings of the Royal Society B: Biological Sciences 286 (2019).

[18] T. Vicsek and A. Zafeiris, Collective motion, Physics Report 517 (2012) 71-140.

[19] R. Engeszer, G. Wang, M. Ryan, and D. Parichy, Sex-specific perceptual spaces for a vertebrate basal social aggregative behavior, Proceedings of the National Academy of Sciences of the United States of America (2008).

[20] S. Rogers, D. Cullen, M. Anstey, M. Burrows, e. Despland, T. Dudgson, T. Matheson, S. Ott, K. Stettin, G. Sword, and S. Simpson, Rapid behavioural gregarization in the desert locust, Schistocerca gregaria entails synchronous changes in both activity and attraction to conspecifics, Journal of Insect Physiology 65 (2014) 9-26.

[21] S. Ott and S. Rogers, Gregarious desert locusts have substantially larger brains with altered proportions compared with the solitarious phase, Proceedings of the Royal Society B: Biological Sciences (2010) 3087-3096.

[22] S. Sakai, B. Arsznov, B. Lundrigan, and K. Holekamp, Brain size and social complexity: A computed tomography study in hyaenidae, Brain, Behavior and Evolution 77 (2011) 91-104.

[23] B. Arsznov and S. Sakai, Pride diaries: Sex, brian size and sociality in the African lion (Panthera leo) and cougar (Puma concolor), Brain, Behavior and Evolution 79 (2012) 275-289.

[24] F. Peréz-Barbería and I. Gordon, Gregariousness increases brain size in ungulates, Oecologica 145 (2005) 41-52. 
[25] R. Dunbar, Neocortex size as constraint on group size in primates, Journal of Human Evolution 22 (1992) 469-493.

[26] J. Angulo, D. Printz, M. Ledoux, and B. McEwen, Isolation stress increases tyrosine hydroxylase $m R N A$ in the locus coeruleus and midbrian and decreases proenkephalin mRNA in the striatum and nucleus accumbens, Molecular Brain Research 11 (1991) 301-308.

[27] E. Dong, K. Matsumoto, M. Tohda, Y. Kaneko, and H. Watanabe, Diazepam binding inhibitor (DBI) gene expression in the brains of socially isolated and group-housed mice, Neuroscience Research 33 (1999) 171-177.

[28] E. Del-Bel, S. Mia, O. Lourenç, R. Joca, C. Udia, M. Padovan, and F. Guimara-Es, Effects of isolatoin-rearing on sertonin-1A and M1-muscarinic receptor messenger RNA expression in the hippocampal formation of rats, Neuroscience Letters 332 (2002) 123-126.

[29] R. Pascual, S. Zamora-León, and A. Valero-Cabré, Effects of postweaning social isolation and re-socialization on the expression of vasoactive intestinal peptide (VIP) and dendritic development in the medial prefrontal cortex of the rat, Acta Neurobiologiae Experimentae (2006) 7-14.

[30] J. Levine, R. Youngs, M. MacDonalds, M. Chu, A. Leeder, F. Berthiaume, and C. Konradi, Isolation rearing and hyperlocomotion are associated with reduced immediate early gene expression levels in the medial prefrontal cortex, Neuroscience 145 (2007) 42-55.

[31] J. Lukkes, C. Summers, J. Scholl, K. Renner, and G. Forster, Early life social isolation alters corticotropin-releasing factor responses in adult rats, Neuroscience 158 (2009) 845-855.

[32] M. Robinson, D. McCarthy, and G. Smyth, edgeR: a Bioconductor package for differential expression analysis of digital gene expression data, Bioinformatics 26 (2010) 139-140.

[33] G. Hermes, N. Li, C. Duman, and R. Duman, Post-weaning chronic social isolation produces profound behavioral dysregulation with decreases in prefrontal cortex synaptic-associated protein expression in female rats, Physiology and Behavior 104 (2011) 354-359.

[34] Q. Meng, N. Li, X. Han, F. Shao, and W. Wang, Effects of adolescent social isolation on the expression of brain-derived neurotrophic factors in the forebrain, European Journal of Pharmacology 650 (2011) 229-232. 
[35] X. Han, N. Li, X. Xue, f. Shao, and W. Wang, Early social isolation disrupts latent inhibition and increases dopamine $D 2$ receptor expression in the medial prefrontal cortex and nucleus accumbens of adult rats, Brain Research 1447 (2012) 38-43.

[36] K. Matsumoto, K. Ono, H. Ouchi, R. Tsushima, and Y. Murakami, Social isolation stress down-regulates cortical early growth response 1 (Egr-1) expression in mice, Neuroscience Research 73 (2012) 257-262.

[37] V. Wall, E. Fischer, and S. Bland, Isolation rearing attenuates social interaction-induced expression of immediate early gene protein products in the medial prefrontal cortex of male and female rats, Physiology and Behavior 107 (2012) 440-450.

[38] N. Swerdlow, G. Light, R. Trim, M. Breier, S. Hines, and S. Powell, Forebrain gene expression predicts deficits in sensorimotor gating after isolation rearing in male rats, Behavioural Brain Research 57 (2013) 118-128.

[39] R. Araki, S. Nishida, Y. Hiraki, K. Matsumoto, and T. Yabe, DNA methylation of the GC box in the promoter region mediates isolation rearing-induced suppression of srd5a1 transcription in the prefrontal cortex, Neuroscience Letters 606 (2015) $135-139$.

[40] A. Kumari, P. Singh, M. Baghel, and M. Thakur, Social isolation mediated anxiety like behvior is associated with enhanced expression and regulation of BDNF in the female mouse brain, Physiology and Behavior (2016) 34-42.

[41] C. Ko and Y. Liu, Disruptions of sensorimotor gating, cytokines, glycemia, monoamines, and genes in both sexes of rats reared in social isolation can be ameliorated by oral chronic quetiapine administration, Brain, Behavior, and Immunity 51 (2016) 119-130.

[42] M. Li, W. Du, F. Shao, and W. Wang, Cognitive dysfunction and epigenetic alterations of the BDNF gene are induced by social isolation during early adolescence, Behavioural Brain Research 313 (2016) 177-183.

[43] S. Lander, D. Linder-Shacham, and I. Gaisler-Salomon, Differential effects of social isolationin adolescent and adult mic eon behavior and cortical gene expression, .

[44] A. Haj-Mirzaian, R. Nikbakhsh, K. Ramezanzadeh, M. Rezaee, H. Amini-Khoei, A. Haj-Mirzaian, M. Ghesmati, K. Afshari, N. Haddadi, and A. Dehpour, Involvement of opioid system in behavioral despair induced by social isolation stress in mice, Biomedicine and Pharmacotherapy 109 (2019) 938-944.

[45] H. Harlow, R. Dodsworth, and M. Harlow, Total social isolation in monkeys, Proc Natl Sci USA 54 (1965) 90-97. 
[46] C. Chang, Y. Hsiao, Y. Chen, Y. Yu, and P. Gean, Social isolation-induced increase in NMDA receptors in the hippocampus exacerbates emotional dysregulation in mice, Hippocampus 25 (2015) 474-485.

[47] M. Yilmaz and M. Meiser, Rapid innate defensive responses of mice to looming visual stimuli, Current Biology 23 (2013) 2011-2015.

[48] M. Zelikowsky, M. Hui, T. Karigo, A. Choe, B. Yang, M. R. Blanco, K. Beadle, V. Gradinaru, B. Deverman, and D. Anderson, The Neuropeptide Tac2 Controls a Distributed Brain State Induced by Chronic Social Isolation Stress, Cell 173 (2018) 1265-1268.

[49] J. Cushman, M. Moore, R. Olsen, and M. Fanselow, The role of $\delta$ GABA(A) receptor in ovarian cycle-linked changes in hippocampus-dependent learning and memory, Neurochemical Research 39 (2014) 1140-1146.

[50] T. Kosten, M. Miserendino, J. Bombace, H. Lee, and J. Kim, Sex-selective effects of neonatal isolation on fear conditioning and foot shock sensitivity, Behavioural Brain Research 157 (2005) 235-244.

[51] Á. Tulogdi, M. Tóth, B. Barsvári, L. Biró, É. Mikics, and J. Haller, Effects of resocialization on post-weaning social isolation-induced abnormal aggression and social deficits in rats, .

[52] W. Hamilton, Geometry for the Selfish Herd, Journal for theoretical Biology 31 (1971) 295-311.

[53] I. Vine, Risk of Visual Detection and Pursuit by a Predator and the Selective Advantage of Flocking Behaviour, Journal of theoretical Biology 30 (1971) 405-422.

[54] B. Fairbanks and F. Dobson, Mechanisms of the group-size effect on vigilance in Columbian ground squirrels: diluation versus detection, Animal Behaviour $\mathbf{7 3}$ (2007) 115-123.

[55] M. Schmitt, K. Stears, C. Wilmers, and A. Shrader, Determining the relative importance of dilution and detection for zebra foraging in mixed-species herds, Animal Behaviour 96 (2014) 151-158.

[56] M. Elgar, Predator vigilance and group size in mammals and birds: a critical review of the empirical evidence, Biological Reviews - Cambridge Philosophical Society 64 (1989) 13-33.

[57] J. Wine and F. Krasne, The Organization of Escape Behaviour in the Crayfish, Journal of Experimental Biology 56 (1972). 
[58] P. Curzon, M. Zhang, and R. Radek, The Behavioral Assessment of Sensorimotor Processes in the Mouse: Acoustic Startle, Sensory Gating, Locomotor Activity, Rotarod, and Beam Walking. In: J. J. Buccafusco, editor. Methods of Behavior Analysis in Neuroscience. CRC Press, 2009.

[59] M. Davis, E. Antoniadis, D. Amaral, and J. Winslow, Acoustic startle reflex in rhesus monkeys: A review, Reviews in the Neurosciences 19 (2008) 171-185.

[60] N. Swerdlow, D. Braff, and M. Geyer, Sensorimotor gating of the startle reflex: What we said 25 years ago, what has happened since then, and what comes next, Journal of Psychopharmacology 30 (2016) 1072-1081.

[61] H. Burgess and M. Granato, Sensorimotor gating in larval zebrafish, Journal of Neuroscience 27 (2007) 4984-4994.

[62] S. Shao, M. Li, W. Du, F. Shao, and W. Wang, Galanthamine, an acetylcholine inhibitor, prevents prepulse inhibition deficits induced by adolescent social isolation or MK-801 treatment, Brain Research 1589 (2014) 105-111.

[63] K. Koda, Y. Ago, K. Yano, M. Nishimura, H. Kobayashi, A. Fukada, K. Takuma, and T. Matsuda, Involvement of decreased muscarinic receptor function in prepulse inhibition deficits in mice reared in social isolation, .

[64] I. Machatschke, B. Bauer, L. Glenk, E. Millesi, and B. Wallner, Spatial learning and memory differs between single and cohabitated guinea pigs, Physiology and Behavior 102 (2011) 311-316.

[65] C. Zorzo, M. Méndez-López, M. Méndez, and J. Arias, Adult social isolation leads to anxiety and spatial memory impairment: Brain activity pattern of Cox and c-Fos, Behavioural Brain Research 365 (2019) 170-177.

[66] B. Wang, Q. Wu, L. Lei, H. Sun, N. Michael, X. Zhang, Y. Wang, Y. Zhang, B. Ge, x. Wu, Y. Wang, Y. Xin, J. Zhao, and S. Li, Long-term social isolation inhibits autophagy activation, induces postsynaptic dysfunctions and impairs spatial memory, Experimental Neurology 311 (2019) 213-224.

[67] D. An, W. Chen, D. Yu, S. Wang, W. Yu, H. Xu, D. Wang, D. Zhao, Y. Sun, J. Wu, Y. Wang, and S. Yin, Effects of social isolation, re-socialization and age on cognitive and aggressive behaviors of Kunming mice and BALB/c mice, Animal Science Journal 88 (2017) 798-806.

[68] D. Ibi, K. Takuma, H. Koike, H. Mizoguchi, K. Tsuritani, Y. Kuwahara, H. Kamei, T. Nagai, Y. Yoneda, T. Nabeshima, and K. Yamada, Social ioslation rearing-induced impairment of the hippocampla neurogenesis is associated with deficits in spatial memory and emotion-related behaviors in juvenile mice, Journal of Neurochemistry 105 (2008) 921-932. 
[69] R. Barrientos, D. Sprunger, S. Campeau, E. Higgins, L. Watkins, J. Rudy, and S. Maier, Brain-derived neurotrophic factor mRNA downregulation produced by social isolation is blocked by intrahippocampal interleukin-1 receptor antagonist, .

[70] J. Murínová, N. Hlaváčová, M. Chmelová, and I. Riečanský, The evidence for altered BDNF expression in the brain of rats reared or housed in social isolation: $A$ systematic review, Frontiers in Behavioral Neuroscience 101 (2017).

[71] P. Bekinschtein, M. Cammarota, C. Katche, L. Slipcszuk, J. Rossato, A. Goldin, I. Izquierdo, and J. Medina, BDNF is essential to promote persistence of long-term memory storage, Proceedings of the National Academy of Sciences of the United States of America 105 (2008) 2711-2716.

[72] A. Silva-Gómez, D. Rojas, I. Juárez, and G. Flores, Decreased dendritic spine density on prefrontal cortical and hippocampal pyramidal neurons in postweaning social isolation rats, Brain Research 983 (2003) 128-136.

[73] T. Insel and R. Fernald, How the brain processes social information: Searching for the social brain, Annual Review of Neuroscience 27 (2004) 697-722.

[74] D. Thor, K. Wainwright, and W. Holloway, Persistence of attention to a novel conspecific, Developmental Psychobiology 15 (1982) 1-8.

[75] J. Kogan, P. Frankland, and A. Silva, Long-term memory underlying hippocampus-dependent social recognition in mice, Hippocampus 10 (2000) 47-56.

[76] R. Pena, A. Pereira-Caixeta, M. Moraes, and G. Pereira, Anisomycin administered in the olfactory bulb and dorsal hippocampus impaired social recognition memory consolidation in different time-points, Brain Research Bulletin 109 (2014) 151-157.

[77] K. Richter, G. Wolf, and M. Engelmann, Social recognition memory requires two stages of protein synthesis in mice, Learning and Memory 12 (2005) 407-413.

[78] K. Wanisch, C. Wotjak, and M. Engelmann, Long-lasting second stage of recognition memory consolidation in mice, Behavioural Brain Research 186 (2008) 191-196.

[79] R. Sekiguchi, G. Wolterink, and J. Ree, Short duration of retroactive facilitation of social recognition in rats, Physiology and Behavior 50 (1991) 1253-1256.

[80] H. Shahar-Gold, R. Gur, and S. Wagner, Rapid and Reversible Impairments of Short- and Long-Term Social Recognition Memory are Caused by Acute Isolation of Adult Rats via Distinct Mechanisms, PLoS ONE 8 (2013).

[81] J. Cacioppo, S. Cacioppo, J. Capitanio, and S. Cole, The neuroendocrinology of social isolation, Annual Review of Psychology 66 (2015) 733-767. 
[82] N. Donovan, Q. Wu, D. Rentz, R. Sperling, G. Marshall, and M. Glymour, Loneliness, depression and cognitive function in older U.S. adults, International Journal of Geriatric Psychiatry 32 (2017) 564-573.

[83] B. Yu, A. Steptoe, Y. Chen, and X. Jia, Social isolation, rather than loneliness, is associated with cognitive decline in older adults: The China Health and Retirement Longitudinal Study, Psychological Medicine (2020) 1-8.

[84] E. Lara, F. Caballero, L. Rico-Uribe, B. Olaya, J. Haro, J. Ayuso-Mateos, and M. Miret, Are loneliness and social isolation associated with cognitive decline?, International Journal of Geriatric Psychiatry 34 (2019) 1613-1622.

[85] J. I. Webster, L. Tonelli, and E. M. Sternberg, Neuroendocrine Regulation of Immunity, Annual Reviews of Immunology 20 (2002) 125-163.

[86] M. A. Sacta, Y. Chinenov, and I. Rogatsky, Glucocorticoid Signaling: An Update from a Genomic Perspective, Annual Reviews of Physiology 78 (2016) 155-180.

[87] J. W. Funder, Glucocorticoid and Mineralocorticoid Receptors: Biology and Clinical Relevance, Annual Reviews of Medicine 48 (1997) 231-240.

[88] R. Sapolsky, S. Alberts, and J. Altmann, Hypercortisolism Associated with Social Subordinance or Social Isolation Among Wild Baboons, Arch Gen Psychiatry 54 (1997) 1137-1143.

[89] A. Blecher, N. Bennett, K. Medger, N. Hagenah, A. Ganswindt, and M. Oosthuizen, Effect of colony disruption and social isolation on naked mole-rat endocrine correlates, General and Comparative Endocrinology 295 (2020).

[90] L. Galhardo and R. Oliveira, The effects of social isolation on steroid hormone levels are modulated by previous social status and context in a cichlid fish, Hormones and Behavior 65 (2014) 1-5.

[91] M. Joëls, H. Karst, and R. Sarabdjitsingh, The stressed brain of humans and rodents, Acta Physiologica 223 (2018).

[92] G. Boero, M. Pisu, F. Biggio, L. Muredda, G. Carta, S. Banni, E. Paci, P. Follesa, A. Concas, P. Porcu, and M. Serra, Impaired glucocorticoid-mediated HPA axis negative feedback induced by juvenile social isolation in male rats, Neuropharmacology 133 (2018) 242-253.

[93] Y. Pan, Y. Liu, K. Young, Z. Zhang, and Z. Wang, Post-weaning social isolation alters anxiety-related behavior and neurocemical gene expression in the brain of male prairie voles, Neuroscience Letters 454 (2009) 67-71. 
[94] A. Giacomini, M. de Abreu, G. Kaokoski, R. Idalêncio, F. Kalichak, T. Oliveira, J. da Rosa, D. Gusso, A. Piato, and L. Barcellos, My stress, our stress: Blunted cortisol response to stress in isolated housed zebrafish, year $=$, .

[95] B. Lindsey and V. Tropepe, Changes in the social environment induce neurogenic plasticity predominantly in niches residing in sensory structures of the zebrafish brain independently of cortisol levels, Developmental Neurobiology 74 (2014) 1053-1077.

[96] C. Koch, B. Leinweber, B. Drengberg, C. Blaum, and H. Oster, Interaction between circadian rhythms and stress, Neurobiology of Stress 6 (2017) 57-67.

[97] R. Klinke, H.-C. Pape, A. Kurtz, and S. Silbernagl, eds., Physiologie, ch. Hören und Sprechen: Kommunikation des Menschen. Thieme, 2005.

[98] M. Lapiz, A. Fulford, S. Muchimapura, R. Mason, T. Parker, and C. Marsden, Influence of postweaning social isolation in the rat on brain development, conditioned behavior, and neurotransmission, Neuroscience and Behavioral Physiology 33 (2003) 13-29.

[99] D. Wallace, M. Han, D. Graham, T. Green, V. Vialou, S. liguez, J. Cao, A. Kirk, S. Chakravarty, A. Kumar, V. Krishnan, R. Neve, D. Cooper, C. Bolãos, M. Barrot, C. McClung, and E. Nestler, CREB regulation of nucleus accumbens excitability mediates social isoation-induced behavioral deficits, Nature Neuroscience 12 (2009) 200-209.

[100] F. Scale, M. Nenov, E. Crofton, A. Singh, O. Folorunso, Y. Zhang, B. Chesson, N. Wildburger, T. James, M. Alshammari, T. Alshammari, H. Elfrink, C. Grassi, J. Kasper, A. Smith, J. Hommel, C. Lichti, J. Rudra, M. D'Ascenzo, T. Green, and F. Laezza, Environmental Enrichment and Social Isolation Mediate Neuroplasticity of Medium Spiny Neurons through the GSK3 Pathway, Cell Reports 28 (2018) 555-567.

[101] M. Serra, M. Pisu, M. Littera, G. Papi, E. Sanna, F. Tuveri, L. Usala, R. Purdy, and G. Biggio, Social isolation-induced decreases in both the abundance of neuroactive steroids and $G A B A(A)$ receptor function in rat brain, Journal of Neurochemistry 75 (2000) 732-740.

[102] D. Reddy, Progress in Brain Research, ch. Neurosteroids. Endogenous role in the human brain and therapeutic potentials, pp. 113-137. Elsevier, 2010.

[103] M. Nin, L. Martinze, F. Piribi, M. Nelson, and G. Pinna, Neurosteroids reduce social isolation-induced behavioral deficits: A proposed link with neurosteroid-mediated upregulation of BDNF expression, Frontiers in Endocrinology (2011). 
[104] A. Locci, P. Geoffroy, M. Miesch, A. Mensah-Nyagan, and G. Pinna, Social Isolation in Early versus Late Adolescent Mice is Associated with Persistent Behavioral Deficits that can be improved by Neurosteroid-based Treatment, Frontiers in Cellular Neuroscience 208 (2017).

[105] G. Matthews, E. Nieh, C. Vander Weele, S. Halbert, R. Pradhan, A. Yosafat, G. Glober, E. Izadmehr, R. Thomas, G. Lacy, C. Wildes, M. Ungless, and K. Tye, Dorsal Raphe Dopamine Neurons Represent the Experience of Social Isolation, Cell 164 (2016) 617-631.

[106] C. Heidbreder, I. Weiss, A. Domeney, C. Pryce, J. Homberg, G. Hedou, J. Feldon, M. Moran, and P. Nelson, Behavioral, neurochemical and endocrinological characterization of the early social isolation syndrome, Neuroscience 100 (2000) 749-768.

[107] E. O. Wilson, Sociobiology: the new synthesis. Belknap Press of Harvard University Press, 25th ed., 2000.

[108] G. Robinson, C. Grozinger, and C. Whitfield, Sociogenomics: Social life in molecular terms, Nature Reviews Genetics 6 (2005) 257-270.

[109] M. Cservenák, V. Kis, D. Keller, D. Dimén, L. Menyhárt, S. Oláh, E. Szabó, J. Barna, E. Renner, T. Usdin, and A. Dobolyi, Maternally involved galanin neurons in the preoptic area of the rat, Brain Structure and Function 222 (2017) 781-798.

[110] H. Tunbak, M. Vazquez-Prada, T. Ryan, A. Kampff, and E. Dreosti, Whole-brain mapping of socially isolated zebrafish reveals that lonely fish are not loners, eLife 9 (2020).

[111] J. George, Z. Bell, D. Condliffe, K. Dohrer, T. Abaurrea, K. Spencer, A. Leitão, M. Gahr, P. Hurd, and D. Clayton, Acute social isolation alters neurogenomic state in songbird forebrain, Proceedings of the National Academy of Sciences (2019).

[112] K. Northcutt and J. Lonstein, Social contact elicits immediate-early gene expression in dopaminergic cells of the male prairie vole extended olfactory amygdala, .

[113] S. Burmeister, E. Jarvis, and R. Fernald, Rapid behavioral and genomic responses to social opportunity, PLoS Biology 3 (2005) 1996-2004.

[114] C. Nyman, S. Fischer, N. Aubin-Horth, and B. Tabosrky, Evolutionary conserved neural signature of early life stress affects animal social competence, Proceedings of the Royal Society B: Biological Sciences 285 (2018).

[115] S. Cole, L. Hawkley, J. Arevalo, C. Sung, R. Rose, and J. Cacioppo, Social regulation of gene expression in human leukocytes, Genome Biology 8 (2007). 
[116] Y. Idaghour, W. Czika, K. Shianna, S. Lee, P. Visscher, H. Martin, K. Miclaus, S. Jadallah, D. Goldstein, R. Wolfinger, and G. Gibson, Geographical genomics of human leukocyte gene expression variation in southern Morocco, Nature Genetics 42 (2010) 62-67.

[117] S. Cole, L. Hawkley, J. Arevalo, and J. Cacioppo, Trancript origin analysis identifies antigen-presenting cells as primary targets of socially regulated gene expression in leukocytes, Proceedings of the National Academy of Sciences of the United States of America 108 (2011) 3080-3085.

[118] M. Irwin and S. Cole, Reciprocal regulation of the neural and innate immune systems, Nature Reviews Immunology 11 (2011) 625-632.

[119] G. Miller, E. Chen, A. Fok, H. Walker, A. Lim, E. Nicholls, S. Cole, and M. Kobor, Low early-life social class leaves a biological residue manifested by decreased glucocorticoid and increased proinflammatory signaling, Proceedings of the National Academy of Sciences of the United States of America 106 (2009) 14716-14721.

[120] M. O'Connor, C. Schultze-Florey, M. Irwin, J. Arevalo, and S. Cole, Divergent gene expression responses to Complicated Grief and Non-complicated Grief, Brain, Behavior, and Immunity 37 (2014) 78-83.

[121] S. Cole, Human Social Genomics, PLoS Genetics 10 (2014) e1004601.

[122] F. Althammer, G. Jirikowski, and V. Grinevich, The oxytocin system of mice and men - Similarities and discrepancies of oxytocinergic modulation in rodents and primates, Peptides 109 (2018) 1-8.

[123] B. Harvey, W. Regenass, W. Dreyer, and M. Möller, Social isolation rearing-induced anxiety and response to agomelatine in male and female rats: Role of corticosterone, oxytocin, and vasopressin, Journal of Psychopharmacology 33 (2019) 640-646.

[124] A. Ross, K. McCann, T. Larkin, Z. Song, Z. Grieb, K. Huhman, and H. Albers, Sex-dependent effects of social isolation on the regulation of arginine-vasopressin (AVP) V1a, oxytocin (OT) and serotonin (5HT) 1a receptor binding and aggression, Hormones and Behavior 116 (2019).

[125] H. Pournajafi-Nazarloo, W. Kenkel, S. Mohsenpour, L. Sanzenbacher, H. Saadat, L. Partoo, J. Yee, F. Azizi, and C. Carter, Exposure to chronic isolation modulates receptors $m R N A$ s for oxytocin and vasopressin in th ehypothalamus and heart, Peptides 43 (2013) 20-26. 
[126] K. Asahina, K. Watanabe, B. Duistermars, E. Hoopfer, C. González, e. Eyjólfsdóttir, P. Perona, and D. Anderson, Tachykinin-expressing neurons control male-specific aggressive arousal in drosophila, Cell 156 (2014) 221-235.

[127] F. Reichmann and P. Holzer, Neuropeptide Y: A stressful review, Neuropeptides 55 (2016) 99-109.

[128] J. White, Neuropeptide Y: a central regulator of energy homeostasis, .

[129] A. Thorsell, C. Slawecki, A. El Khoury, A. Mathe, and C. Ehlers, The effects of social isolation on neuropeptide $Y$ levels, exploratory and anxiety-related behaviors in rats, .

[130] R. Krolow, C. Noschang, D. Arcego, A. Huffell, M. Marcolin, A. Benitz, C. Lampert, R. Fitarelli, and C. Dalmaz, Sex-specific effects of isolation stress and consumption of palatable diet during the prepubertal period on metabolic parameters, Metabolism: Clinical and Experimental 62 (2013) 1268-1278.

[131] J. George, Z. Bell, D. Condliffe, T. Dohrer, K. an dAbaurrea, K. Spencer, A. Leitão, M. Gahr, P. Hurd, and D. Clayton, Acute social isolation alters neurogenomic state in songbird forebrain, Proceedings of the National Academy of Sciences of the United States of America 117 (2020) 23311-23316.

[132] Z. Bell, P. Lovell, C. Mello, P. Yip, J. George, and D. Clayton, Urotensin-related gene transcripts mark developmental emergence of the male forebrian vocal control system in songbirds, Scientific Reports 9 (2019).

[133] P. Agrawal, D. Kao, P. Chung, and L. Looger, The neuropeptide Drosulfakinin regulates social isolation-induced aggression in Drosophila, Journal of Experimental Biology 223 (2020).

[134] J. Balcombe, What a fish knows. Oneworld Publications, London, 2016.

[135] R. Fernald, Social control of the brain, Annual Review of Neuroscience 35 (2012) 133-151.

[136] D. Radakov, Schooling in the ecology of fish. Mill H. Halsted Press, 1973.

[137] F. Hinz, M. Aizenberg, G. Tushev, and E. Schuman, Protein synthesis-dependent associative long-term memory in larval zebrafish, .

[138] R. Hinz and G. De Polavieja, Ontogeny of collective behavior reveals a simple attraction rule, Proceedings of the National Academy of Sciences of the United States of America 114 (2017) 2295-2300.

[139] E. Dreosti, G. Lopes, A. Kampff, and S. Wilson, Development of social behavior in young zebrafish, Frontiers in Neural Circuits 9 (2015). 
[140] N. Miller and R. Gerlai, From Schooling to Shoaling: Patterns of Collective Motion in Zebrafish (Danio rerio), PLoS ONE 7 (2012).

[141] G. S. Glenn, J. Kauffman, X. Wang, R. Stewart, J. L. Moore, C. J. Kasales, E. Demidenko, and K. C. Cheng, Life Spans and Senescent Phenotypes in Two Strains of Zebrafish (Danio Rerio), Experimental Gerontology 37 (2002) 1055-1068.

[142] S. Easter and G. Nicola, The development of vision in the zebrafish (Danio rerio), Developmental Biology 180 (1996) 646-663.

[143] C. B. Kimmel, W. W. Ballard, S. R. Kimmel, B. Ullmann, and T. F. Schilling, Stages of embryonic development of the zebrafish, Developmental Dynamics 203 (1995) 253-310.

[144] A. Miri, K. Daie, A. Arrenberg, H. Baier, E. Aksay, and D. Tank, Spatial gradients and multidimensional dynamics in a neural integrator circuit, Nature Neuroscience 14 (2011) 1150-1161.

[145] F. Kubo, B. Hablitzel, M. Dal Maschio, W. Driever, H. Baier, and A. Arrenberg, Functional architecture of an optic flow-responsive area that drives horizontal eye movements in zebrafish, Neuron 81 (2014) 1344-1359.

[146] M. Orger and H. Baier, Channeling of red and green cone inputs to the zebrafish optomotor response, Visual Neuroscience 22 (2005) 275-281.

[147] E. Naumann, J. Fitzgerald, T. Dunn, J. Rihel, H. Sompolinsky, and F. Engert, From Whole-Brain Data to Functional Circuit Models: The Zebrafish Optomotor Response, Cell 167 (2016) 947-960.

[148] K. Huang, M. Ahrens, T. Dunn, and F. Engert, Spinal projection neurons control turning behaviors in zebrafish, Current Biology 23 (2013) 1566-1573.

[149] T. Dunn, Y. Mu, S. Narayan, O. Randlett, E. Naumann, C. Yang, A. Schier, J. Freeman, F. Engert, and M. Ahrens, Brain-wide mapping of neural activity controlling zebrafish exploratory locomotion, eLife (2016).

[150] S. Wolf, A. Dubreuil, T. Bertone, U. Böhm, V. Bormuth, R. Candelier, S. Karpenko, D. Hildebrand, I. Bianco, R. Monasson, and G. Debrégeas, Sensorimotor computation underlying phototaxis in zebrafish, Nature Communication 8 (2017).

[151] P. Balm and D. Groneveld, The Melanin-Concentrating Hormone System in Fish, Annals of the New York Academy of Sciences 839 (1998) 205-209.

[152] S. Neuhauss, O. Biehlmaier, M. Seeliger, T. Das, K. Kohler, W. Harris, and H. Baier, Genetic disorders of vision revealed by a behavioral screen of 400 essential loci in zebrafish, Journal of NeurosciencE 19 (1999) 8603-8615. 
[153] I. Bianco, A. Kampff, and E. F., Prey capture behavior evoked by simple visual stimuli in larval zebrafish, Frontiers in Systems Neuroscience (2011).

[154] D. Prober, S. Zimmerman, B. Myers, B. McDermott, S. Kim, S. Caron, J. Rihel, L. Solnica-Krezel, D. Julius, A. Hudspeth, and A. Schier, Zebrafish TRPA1 channels are required for chemosensation but not for thermosensation or mechanosensory hair cell function, Journal of Neuroscience 28 (2008) 10102-10110.

[155] A. Vitebsky, R. Reyes, M. Sanderson, W. Michel, and K. Whitlock, Isolation and characterization of the laure olfactory behavioral mutant in the zebrafish, Danio rerio, Developmental Dynamics 234 (2005) 229-242.

[156] S. Lindsay and R. Vogt, Behavioral responses of newly hatched zebrafish (Danio rerio) to amino acid chemostimulants, Chemical Senses 29 (2004) 93-100.

[157] O. R. Brauchbach, H.-D. Wood, S. Gadbois, A. fine, and R. P. Croll, Olfactory conditioning in the zebrafish (Danio rerio), Behavioural Brain Research 198 (2009) 190-198.

[158] G. Gerlach and N. Lysiak, Kin recognition and inbreeding avoidance in zebrafish, Danio rerio, is based on phenotype matching, Animal Behavior 71 (2006) 1371-1377.

[159] A. Gerlach, G. nd Hodgins-Davis, c. Avolio, and C. Schunter, Kin recognition in zebrafish: a 24-hour window for olfactory imprinting, Proceedings of the Royal Society B: Biological Sciences 275 (2008) 2165-2170.

[160] C. Hinz, R. Namekawa, J. Behrmann-Godel, C. Oppelt, A. Jaeschke, A. Müller, R. Friedrich, and G. Gerlach, Olfactory imprinting is triggered by MHC peptide ligands, Scientific Reports 3 (2013).

[161] D. Biechl, K. Tietje, G. Gerlach, and M. Wullimann, Crypt cells are involved in kin recognition in larval zebrafish, Scientific Reports 6 (2016).

[162] S. Mahabir and R. Gerlai, The Importance of Holding Water: Salinity and Chemosensory Cues Affect Zebrafish Behavior, Zebrafish 14 (2017) 444-458.

[163] S. Korsching, S. Argo, H. Campenhausen, R. Friedrich, A. Rummrich, and F. Weth, Olfaction in zebrafish: What does a tiny teleost tell us?, Seminars in Cell and Developmental Biology 8 (1997) 181-187.

[164] K. Howe, M. Clark, C. Torroja, J. Torrance, C. Berthelot, M. Muffato, et. al., The zebrafish reference genome sequence and its relationship to the human genome, Nature 496 (2013) 498-503. 
[165] P. Haffter, M. Granato, M. Brand, M. C. Mullins, M. Hammerschmidt, D. A. Kane, J. Odenthal, F. J. van Eden, Y. J. Jiang, C. P. Heisenberg, R. N. Kelsh, M. Furutani-Seiki, E. Vogelsang, D. Beuchle, U. Schach, C. Fabian, and C. Nusslein-Volhard, The Identification of Genes with Unique and Essential Functions in the Development of the Zebrafish, Danio Rerio, Development 123 (1996) 1-36.

[166] A. Nasevicius and S. Ekker, Effective targeted gene 'knockdown' in zebrafish, Nature Genetics 26 (2000) 216-220.

[167] K. Kawakami and S. A., Identification of the Tol2 transposase of the medaka fish Oryzias latipes that catalyzes excision of a nonautonomous Tol2 element in zebrafish Danio rerio, .

[168] M. Saif, D. Chatterjee, C. Buske, and R. Gerlai, Sight of conspecific images induces changes in neurochemistry in zebrafish, Behavioural Brain Research 243 (2013) 294-299.

[169] A. Groneberg, J. Marques, A. Martins, R. del Corral, G. de Polavieja, and M. Orger, Early-Life Social Experience Shapes Social Avoidance Reactions in Larval Zebrafish, Current Biology 30 (2020) 1-13.

[170] A. Facciol and R. Gerlai, Zebrafish Shoaling, Its Behavioral and Neurobiological Mechanisms, and Its Alteration by Embryonic Alcohol Exposure: A Review, Frontiers in Behavioral Neuroscience 174 (2020).

[171] J. Herbert-Read, E. Rosén, A. Szorkovszky, C. loannou, B. Rogell, A. Perna, I. Ramnarine, A. Kotrschal, N. Kolm, J. Krause, and D. Sumpter, How predation shapes the social interaction rules of shoaling fish, Proceedings of the Royal Society B: Biological Sciences (2017).

[172] N. Speedie and R. Gerai, Alarm substance induced behavioral responses in zebrafish (Danio rerio), Behavioural Brain Research 188 (2008) 168-177.

[173] R. Abril-de Abreu, A. Cruz, and R. Oliveira, Social dominance modulates eavesdropping in zebrafish, Royal Society Open Science 2 (2015).

[174] M. Teles, S. Cardoso, and R. Oliveira, Social plasticity relies on different neuroplasticity mechanisms across the brain social decision-making network in zebrafish, Frontiers in Behavioral Neuroscience (2016).

[175] K. Maruska, M. Soares, M. Lima-Maximino, H. de Siqueira-Silva, and C. Maximino, Social plasticity in the fish brain: Neuroscientific and ethological aspects, Brain Research 1711 (2019) 156-172. 
[176] K. Clements, T. Miller, J. Keever, A. Hall, and F. Issa, Social status-related differences in motor activity between wild-type and mutant zebrafish, Biological Bulletin 235 (2018) 71-82.

[177] G. Paull, A. Filby, H. Giddins, T. Coe, P. Hamilton, and C. Tyler, Dominance hierarchies in zebrafish (Danio rerio) and their relationship with reproductive success,

[178] E. Gonçalves-De-Freitas and T. C. Mariguela, Social isolation and aggressiveness in the Amazonian juvenile fish Astronotus ocellatus, Brazilian Journal of Biology 66 (2006) 232-238.

[179] S. Hesse and T. Thünken, Growth and social behavior in a cichlid fish are affected by social rearing environment and kinship, Naturwissenschaften 101 (2014) 273-283.

[180] T. Ichihashi, Y. Ichikawa, and T. Matsushima, A non-social and isolate rearing condition induces an irreversible shift toward continued fights in the male fighting fish (Betta splendens), Zoological Science 21 (2004) 723-729.

[181] D. Franck, R. Hannes, H. Lanffermann, and A. Ribowski, Effects of social isolation on aggressiveness in fish with special reference to the swordtail (Xiphophorus helleri), Behavioural Processes 10 (1985) 415-427.

[182] M. Ausas, L. Mazzitelli-Fuentes, F. Roman, S. Crichigno, A. De Vincenti, and L. Mongiat, Social isolation impairs active avoidance performance and decreases neurogenesis in the dorsomedial telencephalon of the rainbow trout, Physiology and Behavior 198 (2019) 1-10.

[183] K. Dunlap, E. McCarthy, and D. Jashari, Electrocommunication signals alone are sufficient to increase neurogenesis in the brain of adult electric fish, Apteronotus leptorhynchus, Developmental Neurobiology 68 (2008) 1420-1428.

[184] S. Shams, D. Chatterjee, and R. Gerlai, Chronic social isolation affects thigmotaxis and whole-brian serotonin levels in adult zebrafish, Behavioural Brain Research 292 (2015) 283-287.

[185] S. Shams, S. Amlani, C. Buske, D. Chatterjee, and R. Gelai, Developmental social isolation affects adult behavior, social interaction, and dopamine metabolite levels in zebrafish, Developmental Psychobiology 60 (2018) 43-56.

[186] D. Zellner, B. Padnos, D. Hunter, R. MacPhail, and S. Padilla, Rearing conditions differentially affect the locomotor behavior of larval zebrafish, but not their response to valproate-induced developmental neurotoxicity, Neurotoxicology and Teratology 33 (2011) 674-679. 
[187] H. Bleckmann and Z. R., Lateral line system of fish, Integrative zoology 4 (2009) 13-25.

[188] J. Butler and K. Maruska, Mechanosensory signaling as a potential mode of communication during social interactions in fishes, The Journal of Experimental Biology 219 (2016) 2781-2789.

[189] D. McPherson, Sensory hair cells: An introduction to structure and physiology, .

[190] P. Görner, Untersuchungen zur Morphologie und Elektrophysiolgie des Seitenlinienorgans vom Krallenfrosch (Xenopus laevis Daudin), Zeitschrift für vregleichende Physiologie 47 (1963) 316-338.

[191] S. Coombs, J. New, and M. Nelson, Information-processing demands in electrosensory and mechanosensory lateral line systems, Journal of Physiology 96 (2002) 341-354.

[192] P. Pichler and L. Lagnado, Motor Behavior Selectively Inhibits Hair Cells Activated by Forward Motion in the Lateral Line of Zebrafish, Current Biology 30 (2020) 150-157.

[193] N. Gompel, N. Cubedo, C. Thisse, B. Thisse, A. C. Dambly-Chaudie, and A. Ghysen, Pattern formation in the lateral line of zebrafish, Mechanisms of Development 105 (2001) 69-77.

[194] J. New and S. Singh, Central Topography of Anterior Lateral Line Nerve Projections in the Channel Catfish, Ictalurus punctatus, Brain, Behavior and Evolution 43 (1994) 34-50.

[195] D. Alexandre and A. Ghysen, Somatotopy of the lateral line projection in larval zebrafish, Proceedings of the National Academy of Sciences of the United States of America 96 (1999) 7558-7562.

[196] H. Bleckmann and J. Mogdans, The Lateral Line System, ch. Central Processing of Lateral Line Information, pp. 253-280. Springer, 2013.

[197] J. Plachta, J. Mogdans, and H. Bleckmann, Responses of midbrain lateral line units of the goldfish, Carassius auratus, to constant-amplitude and amplitude-modulated water wave stimuli, Journal of Comparative Physiology A 185 (1999) 404-417.

[198] H. Bleckmann and R. Zelick, The responses of peripheral and central mechanosensory lateral line units of weakly electric fish to moving objects, Journal of Comparative Physiology A 172 (1993) 115-128. 
[199] M. Satou, H. Takeuchi, J. Nishii, M. Tanabe, S. Kitamura, N. Okumoto, and M. Iwata, Behavioral and electrophysiological evidences that the lateral line is involved in the inter-sexual vibrational communication of the himé salmon (landlocked red salmon, Oncorhynchus nerka), Journal of Comparative Physiology A 174 (1994) 539-549.

[200] E. Neher and B. Sakmann, Single-channel currents recorded from membrane of denervated frog muscle fibres, Nature 260 (1976) 799-802.

[201] A. Hodgkin and A. Huxley, Action potentials recorded from inside a nerve fibre, Nature 144 (1939) 710-711.

[202] E. Marder, Neuromodulation of Neuronal Circuits: Back to the Future, .

[203] M. Nusbaum, D. Blitz, and E. Marder, Functional consequences of neuropeptide and small-molecule co-transmission, Nature Reviews Neuroscience 18 (2017) 389-403.

[204] G. Jékely, S. Melzer, I. Beets, I. Kadow, J. Koene, S. Haddad, and L. Holden-Dye, The long and the short of it - A perspective on peptidergic regulation of circuits and behaviour, Journal of Experimental Biology 221 (2018).

[205] V. Hook, L. Funkelstein, D. Lu, S. Bark, J. Wegrzyn, and S. Hwang, Proteases for Processing Proneuropeptides into Peptide Neurotransmitters and Hormones, Annual Review of Pharmacology and Toxicology 48 (2008) 393-423.

[206] G. Jékely, Global view of the evolution and diversity of metazoan neuropeptide signaling, Proceedings of the National Academy of Sciences of the United States of America 110 (2013) 8702-8707.

[207] M. Elphick, O. Mirabeau, and D. Larhammar, Evolution of neuropeptide signalling systems, Journal of Experimental Biology 221 (2018).

[208] A. Swensen and E. Marder, Multiple peptides converge to activate the same voltage-dependent current in a central pattern-generating circuit, Journal of Neuroscience 20 (2000) 6752-6759.

[209] S. Haddad and E. Marder, Circuit Robustness to Temperature Perturbation is Altered by Neuromodulators, .

[210] M. De Bono, D. Tobin, M. Davis, L. Avery, and C. Bargmann, Social feeding in Caenorhabditis elegans is induced by neurons that detect aversive stimuli, Nature 419 (2002) 899-903.

[211] R. Graul and W. Sadee, Evolutionary relationships among G protein-coupled receptors using a clustered database approach, AAPS PharmSci 3 (2001) 25-42.

[212] A. Harmar, Family-B G-protein-coupled receptors, Genome Biology 2 (2001). 
[213] F. Jamen, K. Persson, G. Bertrand, N. Rodriguez-Henche, R. Puech, J. Bockaert, B. Ahrén, and P. Brabet, PAC1 receptor-deficient mice display impaired insulinotropic response to glucose and reduced glucose tolerance, Journal of Clinical Investigation 105 (2000) 1307-1315.

[214] K. Lewis, C. Li, M. Perrin, A. Blount, K. Kunitake, C. Donaldson, J. Vaughan, T. Ryes, J. Gulyas, W. Fischer, L. Bilezikjian, J. Rivier, P. Sawchenko, and W. Vale, Identification of urocortin III, an additional member of the corticotropin-releasing factor (CRF) family with high affinity for the CRF2 receptor, Proceedings of the National Academy of Sciences of the United States of America 98 (2001) 7570-7575.

[215] M. Bastepe, S. Turan, and Q. He, Heterotrimeric G proteins in the control of parathyroid hormone actions, Journal of Molecular Endocrinology 58 (2017).

[216] S. A. Siegelbaum, D. E. Clapham, and J. H. Schwartz, Principles of Neural Science, ch. Modulation of Synaptic Transmission: Second Messengers, pp. 236-259. McGraw Hill, 2013.

[217] T. Usdin, Evidence for a Parathyroid Hormone-2 receptor selective ligand in the hypothalamsu, Endocrinology 138 (1997) 831-834.

[218] A. Dobolyi, H. Ueda, H. Uchida, M. Palkovits, and T. Usdin, Anatomical and physiological evidence for involvement of tuberoinfundibular peptide of 39 residues in nociception, .

[219] A. Eichinger, N. Fiaschi-Taesch, T. Massfelder, S. Fritsch, M. Barthelmebs, and J. Helwig, Transcript expression of the tuberinfundibular peptide (TIP)39/PTH2 receptor system and non-PTH1 receptor-mediated tonic effects of TIP39 and other PTH2 receptor ligands in renal vessels, Endocrinology 143 (2002) 3036-3043.

[220] G. Ross, P. Engel, Y. Abdallah, W. Kummer, and K. Schluter, Tuberoinfundibular peptide of 39 residues: A new mediator of cardiac function via nitric oxide production in the rat heart, Endocrinology 146 (2005) 2221-2228.

[221] A. Dobolyi, M. Palkovits, and T. Usdin, Expression and distribution of tuberoinfundibular peptide of 39 residues in the rat central nervous system, Journal of Comparative Neurology 455 (2003) 547-566.

[222] C. Faber, A. Dobolyi, M. Sleeman, and T. Usdin, Distribution of tuberoinfundibular peptide of 39 residues and its receptor, parathyroid hormone 2 receptor, in the mouse brain, Journal of Comparative Neurology 502 (2007) 563-583.

[223] J. Wang, M. Palkovits, T. Usdin, and A. Dobolyi, Forebrain projections of tuberoinfundibular peptide of 39 residues (TIP39)-containing subparafascicular neurons, Neuroscience 138 (2006) 1245-1263. 
[224] L. M. Coolen, J. G. Veening, D. W. Petersen, and M. T. Shipley, Parvocellular subparafascicular thalamic nucleus in the rat: anatomical and functional compartmentalization, Journal of Comparative Neurology 463 (2003) 117-131.

[225] A. Dobolyi, M. Palkovits, and T. Usdin, The TIP-39-PTH2 receptor system: Unique peptidergic cell groups in the brainstem and their interactions with central regulatory mechanisms, Progress in Neurobiology 90 (2010) 29-59.

[226] Y. Yasui, T. Kayahara, K. Nakano, and N. Mizuno, The subparafascicular thalamic nucleus of the rat receives projection fibers from the inferior colliculus and auditory cortex, Brain Research 537 (1990) 323-327.

[227] M. Palkovits, A. Dobolyi, F. Helfferich, and T. Usdin, Acute loud noise activates TIP39-immunoreactive neurons in the rat brain, Ann. N. Y. Acad. Sci. 1018 (2009) 16-24.

[228] J. Wang, M. Palkovits, T. Usdin, and A. Dobolyi, Afferent connections of the subparafascicular area in rat, Neuroscience 138 (2006) 197-220.

[229] T. Varga, M. Palkovits, T. Usdin, and A. Dobolyi, The medial paralemniscal nucleus and its afferent neuronal connections in rat, Journal of Comparative Neurology 511 (2008) 221-237.

[230] T. Usdin, C. Gruber, and T. I. Bonner, Identification and functional expression of a receptor selectively recognizing parathyroid hormone, the PTH2 receptor, Journal of Biological Chemistry 270 (1995) 15455-15458.

[231] J. Wang, M. Palkovits, M. Rusnak, E. mezey, and T. Usdin, Distribution of parathyroid hormone-2 receptor-like immunoreactivity and messener RNA in the rat nervous system, Neuroscience 100 (2000) 629-649.

[232] P. Bhattacharya, Y. Yan, J. Postlethwait, and D. Rubin, Evolution of the vertebrate pth2 (TIP39) gene family and the regulation of PTH type 2 receptor (pth2r) and its endogenous ligand pth2 by hedgehog signaling in zebrafish development, .

[233] C. J. LaBuda, A. Dobolyi, and T. Usdin, Tuberoinfundibular peptide of 39 residues produces anxiolytic and antidepressant actions, Neuroreport 15 (2004) 881-885.

[234] D. B. Fegley, A. Holmes, T. Riordan, C. A. Faber, J. R. Weiss, S. Ma, S. Batkai, P. Pacher, A. Dobolyi, A. Murphy, M. W. Sleeman, and T. Usdin, Increased fear- and stress-related anxiety-like behavior in mice lacking tuberinfundibular peptide of 39 residues, Genes Brain Behavior 7 (2008) 933-942.

[235] H. L. Ward, C. J. Small, K. G. Murphy, A. R. Kennedy, M. A. Ghatei, and S. R. Bloom, The actions of tuberoinfundibular peptide on the hypothalamo-pituitary axes, Endocrinology 142 (2001) 3451-3456. 
[236] T. Usdin, A. Dobolyi, H. Ueda, and M. Palkovits, Emerging functions for tuberoinfundibular peptide of 39 residues, Trends in Endocrinology \& Metabolism 14 (2003) 14-19.

[237] K. Sugimura, T. Murase, S. Ishizaki, K. Tachikawa, H. Arima, Y. Miura, T. Usdin, and Y. Oiso, Centrally administered tuberoinfundibular peptide of 39 residues inhibits arginine vasopressin release in conscious rats, Endocrinology 144 (2003) 2791-2796.

[238] M. Cservenák, D. Keller, V. Kis, E. Fazekas, H. Öllös, A. Lékó, É. Szabó, É. Renner, T. Usdin, M. Palkovits, and Á. Dobolyi, A thalamo-hypothalamic pathway that activates oxytocin neurons in social contexts in female rats, Endocrinology 158 (2017) 335-348.

[239] M. Cservenák, É. Szabó, I. Bodnár, A. Lékó, M. Palkovits, G. Nagy, T. Usdin, and A. Dobolyi, Thalamic neuropeptide mediating the effects of nursing on lactation and maternal motivation, Psychoneuroendocrinology 38 (2013) 3070-3084.

[240] L. Coutellier, A. Logemann, M. Rusnak, and T. Usdin, Maternal Absence of the Parathyroid Hormone 2 Receptor Affects Postnatal Pup Development, Journal of Neuroendocrinology 23 (2011) 612-619.

[241] A. Schroeder, O. Mueller, S. Stocker, R. Salowsky, M. Leiber, M. Gassmann, S. Lightfoot, W. Menzel, M. Granzow, and T. Ragg, The RIN: An RNA integrity number for assigning integrity values to RNA measurements, BMC Molecular Biology 3 (2006).

[242] A. Dobin, C. Davis, F. Schlesinger, J. Drenkow, C. Zaleski, S. Jha, P. Batut, M. Chaisson, and T. Gingeras, STAR: ultrafast universal RNA-seq aligner, Bioinformatics 29 (2013) 15-21.

[243] Y. Liao, G. Smyth, and W. Shi, featureCounts: an efficient general-purpose program for assigning sequence reads to genomic features, Bioinformatics 30 (2014) 923-930.

[244] S. Durinck, Y. Moreau, A. Kasprzyk, S. Davis, B. De Moor, A. Brazma, and W. Huber, BioMart and Bioconductor. a powerful link between biological databases and microarray data analysis, .

[245] Y. Benjamini and Y. Hochberg, Controlling the False Discovery Rate: A Practical and Powerful Approach to Multiple Testing, Journal of the Royal Statistical Society. Series B (Methodological) 57 (1995) 289-300.

[246] D. Raj, B. Wagner, A. McKenna, s. Pandey, A. klein, J. Shendure, J. Gagnon, and A. Schier, Simultaneous single-cell profiling of lineages and cell types in the vertebrate brain, Nature Biotechnology 36 (2018) 442-450. 
[247] T. Stuart, A. Butler, P. Hoffman, C. Hafemeister, E. Papalexi, W. Mauck, Y. Hao, M. Stoeckius, P. Smibert, and R. Satija, Comprehensive Integration of Single-Cell Data, Cell 177 (2019) 1888-1902.

[248] Z. Yao, T. Nguyen, C. van Velthoven, J. Goldy, A. Sedeno-Cortes, F. Baftizadeh, D. Bertagnolli, T. Casper, K. Crichton, S. Ding, O. Fong, E. Garren, and A. Glandon, A taxonomy of transcriptomic cell types across the isocortex and hippocampal formation, bioRxiv (2020).

[249] M. Hywrylycz, E. Lein, A. Guillozet-Bongaarts, E. Shen, L. Ng, J. Miller, L. Van De Lagemaat, K. Smith, A. Ebbert, Z. Riley, and C. Abajian, An anatomically comprehensive atlas of the adult human brain tanscriptome, Nature 489 (2012) 391-399.

[250] M. Speir, A. Bhaduri, N. Markov, P. Moreno, T. Nowakowski, I. Papatheodorou, A. Pollen, L. Seninge, W. Kent, and M. Haeussler, UCSC Cell Browser: Visualize Your Single-Cell Data, bioRxiv (2020).

[251] D. Alsop and M. M. Vijayan, Development of the corticosteroid stress axis and receptor expression in zebrafish, Regulatory, Integrative and Comparative Physiology 294 (2008) R711-R719.

[252] G. Jefferis, C. Potter, A. Chan, E. Marin, T. Rohlfing, C. Maurer, and L. Luo, Comprehensive Maps of Drosophila Higher Olfactory Centers: Spatially Segregated Fruit and Pheromone Representation, Cell 128 (2007) 1187-1203.

[253] O. Randlett, C. Wee, E. Naumann, O. Nnaemeka, D. Schoppik, J. Fitzgerald, R. Portugues, A. Lacoste, C. Riegler, F. Engert, and A. Schier, Whole-brain activity mapping onto a zebrafish brain atlas, Nature Methods 12 (2015) 1039-1046.

[254] L. Anneser, I. C. Alcantara, A. Gemmer, K. Mirkes, S. Ryu, and E. M. Schuman, The neuropeptide Pth2 dynamically senses others via mechanosensation, Nature 588 (2020) 653-657.

[255] I. Namekawa, N. Moenig, and R. Friedrich, Rapid olfactory discrimination learning in adult zebrafish, .

[256] B. Patterson, A. Abraham, M. Maclver, and D. McLean, Visually guided gradation of prey capture movements in larval zebrafish, Journal of Experimental Biology 216 (2013) 3071-3083.

[257] C. Trivedi and J. Bollmann, Visually driven chaining of elementary swim patterns into a goal-directed motor sequence: $A$ virtual reality study of zebrafish prey capture, Frontiers in Neural Circuits 7 (2013). 
[258] J. Semmelhack, J. Donovan, T. Thiele, E. Kuehn, E. Laurell, and H. Baier, $A$ dedicated visual pathway for prey detection in larval zebrafish, eLife 4878 (2014).

[259] J. Larsch and H. Baier, Biological Motion as an Innate Perceptual Mechanism Driving Social Affiliation, Current Biology 28 (2018) 3523-3532.

[260] C. A. d'Alençon, O. A. Pena, C. Wittmann, V. E. Gallardo, R. A. Jones, F. Loosli, U. Liebel, C. Grabher, and M. L. Allende, A high-throughput chemically induced inflammation assay in zebrafish., BMC Biology 8 (2010) 151.

[261] A. D. Brown, T. D. Mussen, J. A. Sisneros, and A. B. Coffin, Reevaluating the use of aminoglycoside antibiotics in behavioral studies of the lateral line, Hearing Research 272 (2011) 1-4.

[262] M. E. Lush and T. Piotrowski, Sensory hair cell regeneration in the zebrafish lateral line, Developmental Dynamics 243 (2015) 1187-1202.

[263] G. Pisano, S. Mason, N. Dhliwayo, R. Intine, and M. Sarras, An Assay for Lateral Line Regeneration in Adult Zebrafish, Journal of Visualized Experiments 86 (2014).

[264] A.-S. LaMantia, S. L. Pomeroy, and D. Purves, Vital Imaging of Glomeruli in the Mouse Olfactory Bulb, Journal of Neuroscience 12 (1992) 976-988.

[265] U. K. Müller and J. L. van Leeuwen, Swimming of larval zebrafish: ontogeny of body waves and implications for locomotory development, Journal of Experimental Biology 207 (2004) 853-868.

[266] T. Palmér, F. Ek, O. Enqvist, R. Olsson, and K. Åström, Action sequencing in the spontaneous swimming behavior of zebrafish larvae - implications for drug development, Scientific Reports 7 (2017).

[267] P. Langer-Safer, M. Levine, and D. Ward, Immunological methods for mapping genes on Drosophila polytene chromosomes, Proceedings of the National Academy of Sciences of the United States of America 79 (1982) 4381-4385.

[268] G. Lauter, I. Söll, and G. Hauptmann, Multicolor fluorescent in situ hybridization to define abutting and overlapping gene expression in the embryonic zebrafish brain, Neural Development 6 (2011).

[269] T. Hokfelt, O. Johansson, A. Ljungdahl, J. Lundberg, and M. Schultzberg, Peptidergic neurones, Nature 284 (1980).

[270] A. Fernandes, E. Beddows, A. Filippi, and W. Driever, Orthopedia Transcription Factor otpa and otpb Paralogous Genes Function during Dopaminergic and Neuroendocrine Cell Specification in Larval Zebrafish, PLOS ONE 8 (2013). 
[271] U. Herget, A. Wolf, M. Wullimann, and S. Ryu, Molecular neuroanatomy and chemoarchitecture of the neurosecretory preoptic-hypothalamic area in zebrafish larvae, Journal of Comparative Neurology 522 (2014) 1542-1564.

[272] S. Anpilov, Y. Shemesh, N. Eren, H. Harony-Nicolas, A. Benjamin, J. Dine, V. Oliveira, O. Forkosh, S. Karamihalev, R. Hüttl, N. Feldman, R. Berger, A. Dagan, G. Chen, I. Neumann, S. Wagner, O. Yizhar, and A. Chen, Wireless Optogenetic Stimulation of Oxytocin Neurons in a Semi-natural Setup Dynamically Elevates Both Pro-social and Agonistic Behaviors, Neuron 107 (2020) 644-655.

[273] U. Herget, J. Gutierrez-Triana, O. Thula, B. Knerr, and S. Ryu, Single-cell reconstruction of oxytocinergic neurons reveals separate hypophysiotropic and encephalotropic subtypes in larval zebrafish, eNeuro 4 (2017).

[274] E. Kanitz, B. Puppe, M. Tuchscherer, M. Heberer, T. Viergutz, and A. Tuchscherer, A single exposure to social isolation in domestic piglets activates behavioural arousal, neuroendocrine stress hormones, and stress-related gene expression in the brain, Physiology and Behavior (2009) 176-185.

[275] Z. STAFF, Zfin publication: Zfin curated data, 2020.

[276] E. Busch-Nentwich, R. Kettleborough, C. M. Dooley, C. Scahill, I. Sealy, R. White, C. Herd, S. Mehroke, N. Wali, S. Carruthers, A. Hall, J. Collins, R. Gibbons, Z. Pusztai, R. Clark, and D. Stemple, Sanger Institute Zebrafish Mutation Project mutant data submission. ZFIN Direct Data Submission, tech. rep., ZFIN, 2013.

[277] D. M. Gendelman, M. Tischler, and P. Gendelman, A primary acoustic startle circuit: Lesion and stimulation studies, Journal of Neuroscience 2 (1982) 791-805.

[278] G. Marquart, K. Tabor, S. Bergeron, K. Briggman, and H. Burgess, Prepontine non-giant neurons drive flexible escape behavior in zebrafish, PLoS Biology 17 (2019).

[279] A. Clemens, H. Wang, and M. Brecht, The lateral septum mediates kinship behavior in the rat, .

[280] M. Schafer and D. Schiller, Navigating Social Space, Neuron 100 (2018) 476-489.

[281] F. Mumtaz, M. Khan, M. Zubair, and A. Dehpour, Neurobiology and consequences of social isolation stress in animal model - A comprehensive review, Biomedicine and Pharmacotherapy 105 (2018) 1205-1222.

[282] L. Hawkley, S. Cole, J. Capitanio, G. Norman, and J. Cacioppo, Effects of social isolation on glucocorticoid regulation in social mammals, Hormones and Behavior 62 (2012) 314-323. 
[283] M. Parker, M. Millington, F. Combe, and C. Brennan, Housing Conditions Differentially Affect Physiological and Behavioural Stress Responses of Zebrafish, as well as the Response to Anxiolytics, PLoS ONE 7 (2012).

[284] L. O'Connell and H. Hofmann, The Vertebrate mesolimbic reward system and social behavior network: A comparative synthesis, Journal of Comparative Neurology 519 (2011) 3599-3639.

[285] D. Mathews and D. Edwards, Involvement of the ventromedial and anterior hypothalamic nuclei in the hormonal induction of receptivity in the female rat, Physiology and Behavior 19 (1977) 319-326.

[286] R. Bridges, P. Mann, and J. Coppeta, Hypothalamic involvement in the regulation of maternal behaviour in the rat: Inhibitory roles for the ventromedial hypothalamus and the dorsal/anterior hypothalamic areas, Journal of Neuroendocrinology 11 (1999) 259-266.

[287] U. Jürgens, $A$ study of the central control of vocalization using the squirrel monkey, Medical Engineering and Physics 24 (2002) 473-477.

[288] S. Kollack-Walker and S. Newman, Mating and agonistic behavior produce different patterns of Fos immunolabeling in male Syrian hamster brain, Neuroscience 66 (1995) 721-736.

[289] J. Powers, S. Newman, and M. Bergondy, MPOA and BNST lesions in male Syrian hamsters. Differential effects on copulatory and chemoinvestigatory behaviors, Behavioural Brain Research 23 (1987) 181-195.

[290] J. Lukkes, A. Burke, N. Zelin, M. Hale, and C. Lowry, Post-weaning social isolation attenuates c-Fos expression in GABAergic interneurons in the basolateral amygdala of adult female rats, Physiology and Behavior 107 (2012) 719-725.

[291] C. A. McCormick, Central Lateral Line Mechanosensory Pathways in Bony Fish. The Mechanosensory Lateral Line. Springer-Verlag, 1989.

[292] H. Scheich and S. Ebbesson, Inputs to the torus semicircularis in the electric fish Eigenmannia virescens - A horseradish-peroxidase study, Cell and Tissue Research 215 (1981) 531-536.

[293] G. Striedter, Auditory, electrosensory, and mechanosensory lateral line pathways through the forebrain in channel catfishes, The Journal of Comparative Neurology 312 (1991) 311-331.

[294] W. Ma and R. Fay, Neural representations of the axis of acoustic particle motion in nucleus centralis of the torus semicircularis of the goldfish, Carassius auratus, . 
[295] G. Vanwalleghem, L. Heap, and E. Scott, A profile of auditory-responsive neurons in the larval zebrafish brain, Journal of Comparative Neurology 525 (2017) 3031-3043.

[296] C. Carr, L. Maler, W. Heiligenberg, and E. Sas, Laminar organization of the afferent and efferent systems of the torus semicircularis of Gymnotiform fish. Morphological substrates for parallel processing in the electrosensroy system, Journal of Comparative Neurology 203 (1981) 649-670.

[297] W. Metzner and W. Heiligenberg, The coding of signals in the electric communication of the gymnotiform fish Eigenmannia: From electroreceptors to neurons in the torus semicircularis of the midbrain, Journal of Comparative Physiology A 169 (1991) 131-150.

[298] H. Bleckmann, Peripheral and central processing of lateral line information, Journal of Comparative Physiology A 194 (2008) 145-158.

[299] M. Penna, W. Lin, and A. Feng, Temporal selectivity by single neurons in the torus semicircularis of Batrachyla antartandica (Amphibia: Leptodactylidae), Journal of Comparative Physiology A 187 (2001) 901-912.

[300] L. Mangiamele and S. Burmeister, Auditory selectivity for acoustic features that confer species recognition in the túngara frog, Journal of Experimental Biology 214 (2011) 2911-2918.

[301] C. Petersen, M. Timothy, D. Kim, A. Bhandiwad, R. Mohr, J. Sisneros, and P. Forlano, Exposure to Advertisement Calls of Reproductive Competitors Activates Vocal-Acoustic and Catecholaminergic Neurons in the Plainfin Midshipman Fish, Porichtys notatus, PLoS ONE 8 (2013).

[302] M. Satou, H. Takeuchi, K. Takei, T. Hasegawa, N. Okumoto, and K. Ueda, Involvement of vibrational and visual cues in eliciting spawning behaviour in male himé salmon (Landlocked red salmon, Oncorhynchus nerka, Animal Behaviour 35 (1987) 1556-1558.

[303] S. Coombs and J. Janssen, Journal of Behavioral and neurophysiological assessment of lateral line sensitivity in the mottled sculpin, Cottus bairdi, Journal of Comparative Physiology A 167 (1990) 557-567.

[304] K. Martin, A brief history of the "feature detector, Cerebral Cortex 4 (1994) 1-7.

[305] G. Vanwalleghem, K. Schuster, M. Taylor, I. Favre-Bulle, and E. Scott, Brain-wide mapping of water flow perception in zebrafish, Journal of Neuroscience 40 (2020) 4130-4144. 
[306] W. Douglas, Stimulus-section coupling: the concept and clues from chromaffin and other cells., British journal of pharmacology 34 (1968) 453-474.

[307] D. Shakiryanova, M. Klose, Y. Zhou, T. Gu, D. Deitcher, H. Atwood, R. Hewes, and E. Levitan, Presynaptic ryanodine receptor-activated calmodulin kinase II increases vesicle mobility and potentiates neuropeptide release, Journal of Neuroscience $\mathbf{2 7}$ (2007) 7799-7806.

[308] C. Hoover, S. Edwards, S. Yu, M. Kittelmann, J. Richmond, S. Eimer, R. Yorks, and K. Miller, A novel CaM kinase II pathway controls the location of neuropeptide release from Caenorhabditis elegans motor neurons, Genetics 196 (2014) 745-765.

[309] C. Velázquez-Marrero, E. Custer, H. Marrero, S. Ortiz-Miranda, and J. Lemos, Voltage-induced $\mathrm{Ca} 2+$ release by ryanodine receptors causes neuropeptide secretion from nerve terminals, Journal of Neuroendocrinology 32 (2020).

[310] L. MacArthur and L. Eiden, Neuropeptide genes: Targets of activity-dependent signal transduction, Peptides 17 (1996) 721-728.

[311] L. Eiden, Y. Anouar, C. Hsu, L. Arthur, and S. Hahm, Transcription Regulation Coupled to Calcium and Protein Kinase Signaling Systems through TRE-and CRE-Like Sequences in Neuropeptide Genes, Advances in Pharmacology 42 (1997) 264-268.

[312] J. Butler and K. Maruska, The mechanosensory lateral line is used to assess opponents and mediate aggressive behaviors during territorial interactions in an African cichlid fish, Journal of Experimental Biology 218 (2015) 3284-3294.

[313] S. Simpson, E. Despland, B. Hägele, and T. Dodgson, Gregarious behavior in desert locusts is evoked by touching their back legs, Proceedings of the National Academy of Sciences of the United States of America 98 (2001) 3895-3897.

[314] S. Rogers, T. Matheson, E. Despland, T. Dodgson, M. Burrows, and S. Simpson, Mechanosensory-induced behavioural gregarization in the desert locust Schistocerca gregaria, Journal of Experimental Biology 206 (2003) 3991-4002.

[315] D. Levy, T. Tamir, M. Kaufman, A. Parabucki, A. Weissbrod, E. Schneidman, and O. Yizhar, Dynamics of social representation in the mouse prefrontal cortex, Nature Neuroscience 22 (2019) 2013-2022.

[316] I. Borazjani and F. Sotiropoulos, Numerical investigation of the hydrodynamics of anuiliform swimming in the transitional and inertial flow regimes, Journal of Experimental Biology 212 (2009) 576-592. 
[317] V. Mwaffo, P. Zhang, s. Cruz, and M. Porfiri, Zebrafish swimming in the flow: A particle image velocimetry study, PeerJ 2017 (2017).

[318] G. losilevskii and A. Rashkovsky, Hydrodynamics of a twisting slender swimmer, Royal Society Open Science 7 (2020).

[319] A. Dobolyi, M. Cservenák, and L. Young, Thalamic integration of social stimuli regulating parental behavior and the oxytocin system, Frontiers in Neuroendocrinology 51 (2018) 102-115.

[320] A. Dobolyi, E. Dimitrov, M. Palkovits, and T. Usdin, The Neuroendocrine Functions of the Parathyroid Hormone 2 Receptor, Frontiers in Endocrinology 3 (2012).

[321] K. Dumais and A. Veenema, Vasopressin and oxytocin receptor systems in the brain: Sex differences and sex-specific regulation of social behavior, Frontiers in Neuroendocrinology 40 (2016) 1-23.

[322] L. Coutellier and T. Usdin, Enhanced long-term fear memory and increased anxiety and depression-like behavior after exposure to an aversive event in mice lacking TIP39 signaling, Behavioural Brain Research 222 (2011) 265-269.

[323] D. Walker, A. Cunningham, J. Gregory, and E. Nestler, Long-term behavioral effects of post-weaning isolation in males and females, Frontiers in Behavioral Neuroscience 13 (2019) 1-20.

[324] M. Fitzgerald and V. Pickel, Adolescent isolation rearing produces a prepulse inhibition deficit correlated with expression of the NMDA GluN1 subunit in the nucleus accumbens, Brain Structure and Function 223 (2018) 3169-3181.

[325] Y. Bai, H. Liu, B. Huang, M. Wagle, and S. Guo, Identification of environmental stressors and validation of liht preference as a measure of anxiety in larval zebrafish, BMC Neuroscience 17 (2016).

[326] W. Hwang, Y. Fu, D. Reyon, M. Maeder, S. Tsai, J. Sander, R. Peterson, J. Yeh, and J. Joung, Efficient genome editing in zebrafish using a CRISPR-Cas system, Nature Biotechnology 31 (2013) 227-229.

[327] C. Chiu, J. Rihel, D. Lee, C. Singh, E. Mosser, S. Chen, V. Sapin, U. Pham, J. Engle, B. Niles, C. Montz, S. Chakravarthy, S. Zimmerman, K. Salehi-Ashtiani, M. Vidal, A. Schier, and D. Prober, A Zebrafish Genetic Screen Identifies Neuromedin U as a Regulator of Sleep/Wake States, Neuron 89 (2016) 842-856.

[328] P. Mullis, B. Rani Pal, D. Matthews, P. Hindmarsh, P. Philliphs, and D. Dunger, Half-life of exogenous growth hormone following suppression of endogenous growth hormone secretion with somtatostatin in type I (insulin-dependent) diabetes mellitus, Clinical Endocrinology 36 (1992) 255-263. 


\section{Lukas Anneser}

Curriculum Vitae

Education

2016-present PhD, Neuroscience, Goethe Universität \& Max Planck Institute for Brain Research. Frankfurt

2019 MBL Summer School, Neural Systems \& Behavior, Marine Biology Laboratory. Woods Hole

2013-2016 Master of Science, Molecular Neuroscience, Ruprecht Karls University. Heidelberg

2010-2013 Bachelor of Science, Molecular Medicine, Friedrich Alexander University. Erlangen

\section{Research Experience (selected)}

Jan 2017 - PhD Student, MPI fOR Brain Research, Frankfurt.

Present Scientific Advisor: Erin Schuman

May 2015 - Diploma Student (M.Sc.), EMBL, Heidelberg.

Aug 2016 Scientific Advisor: Detlev Arendt

Oct 2014 - Visiting Student, Yale University, New Haven.

Mar 2015 Scientific Advisor: Daniel Colón-Ramos

Mar 2013 - Diploma Student (B.Sc.), Friedrich Alexander University, Erlangen.

Aug 2013 Scientific Advisor: Beate Winner

\section{Awards (selected)}

2020 Heiligenberg Travel Grant - International Society for Neuroethology

2019 Poster Prize - FENS Winterschool Neural control of Instinctive and Innate Behavior

2013 Fellow of the Scientific College - German National Academic Foundation

2011 Scholarship - German National Academic Foundation

\section{Presentations}

Talks

2019 Encoding the social environment in zebrafish. Janelia, Ashburn

Saalburgallee 6 - 60385 Frankfurt am Main

Born September $19^{\text {th }} 1990$ in Bamberg 
2019 Transcriptional response to social isolation in zebrafish. MBL, Woods Hole Posters

Nov 2019 Anneser L, Alcantara IC, Gemmer A, Soojin R, Schuman EM. Brain Transcriptional Dynamics and Social Environment Information. Presented at Workshop on Mechanistic Cognitive Neuroscience. 2019 Oct 27 - Nov 01, Janelia, Ashburn, USA.

Jan 2019 Anneser L, Alcantara IC, Gemmer A, Soojin R, Schuman EM. Effect of social isolation on gene transcription in zebrafish. Presented at FENS-Hertie Winter School: Neural Control of Instinctive and Innate Behavior. 2019 Jan 06 - 12, Obergurgl, Austria.

Nov 2018 Anneser L, Alcantara IC, Gemmer A, Soojin R, Schuman EM. Impact of the social environment on gene expression in zebrafish. Presented at 5th Imaging Structure and Function in the Zebrafish Brain Conference. 2018 Nov 30 - Dec 02, Brighton, UK.

\section{Publications}

Toribio-Florez D, Anneser L, De Oliveira N, Pallandt M, Tunn I, Windel H (2021). Where do early-career researchers stand on Open Science Practices? A survey within the Max Planck Society. Frontiers in Research Metrics and Analytics, DOI: 10.3389/frma.2020.586992

Anneser L, Alcantara IC, Gemmer A, Mirkes K, Ryu S, Schuman EM (2020). The Neuropeptide Pth2 Dynamically Senses Others via Mechanosensation. Nature, 588(7839):653-657.

Mishra HK, Prots I, Havlicek S, Kohl Z, Perez-Branguli F, Boerstler T, Anneser L, et al. (2016). GSK3 $\beta$-dependent dysregulation of neurodvelopment in SPG11-patient iPSC model. Ann. Neurol., 79(5):826-840. 